\title{
Nickel Catalyzed trans-Carboamination across Internal Alkynes to Access Multifunctionalized Indoles
}

\author{
Shrikant D. Tambe, Naeem Iqbal, and Eun Jin Cho* \\ Department of Chemistry, Chung-Ang University \\ 84 Heukseok-ro, Dongjak-gu, Seoul 06974, Republic of Korea \\ E-mail: ejcho@cau.ac.kr (E. J.Cho)
}

\section{Supporting Information}

$\begin{array}{ll}\text { General Considerations } & \text { S-2 }\end{array}$

$\begin{array}{ll}\text { Controlled Experiments } & \text { S-3 }\end{array}$

$\begin{array}{ll}\text { Experimental Procedures } & \text { S-4 }\end{array}$

Analytic Data for Synthesized Compounds $\quad$ S-6

$\begin{array}{ll}\text { NMR Spectra ( }\left({ }^{1} \mathrm{H} \text { NMR and }{ }^{13} \text { C NMR) }\right. & \text { S-25 }\end{array}$ 


\section{General Considerations}

\section{General reagent information}

All commercially available reagents including $\mathrm{Ni}(\mathrm{COD})_{2}$ and acetonitrile were purchased from SigmaAldrich, Alfa Aesar, Combi-Blocks, or Acros Organics chemical companies. 2-(2(Diphenylphosphino)ethyl)pyridine (PyPhos) was synthesized according to the known procedure. ${ }^{S l}$ Flash column chromatography was performed using ZEOCHEM ZEOprep silica gel 60 (60-200 mesh).

Ref S1. Moglie, Y.; González-Soria, M. J.; Martín-García, I.; Radivoy, G.; Alonso, F., Catalyst- and solvent-free hydrophosphination and multicomponent hydrothiophosphination of alkenes and alkynes. Green Chemistry 2016, 18, 4896-4907.

\section{General analytical information}

The synthesized tosylated methyl 2-alkynylanilinoacrylate ( $\mathbf{1}$ and $\mathbf{3}$ ), indole derivatives ( $\mathbf{2}$ and $\mathbf{4}$ ), and carbazole derivatives $(\mathbf{8})$ were characterized by ${ }^{1} \mathrm{H}$ NMR, ${ }^{13} \mathrm{C}$ NMR, and FT-IR spectroscopy. NMR spectra were recorded on a Varian $600 \mathrm{MHz}$ instrument (600 MHz for ${ }^{1} \mathrm{H} \mathrm{NMR}$ and $151 \mathrm{MHz}$ for ${ }^{13} \mathrm{C}$ NMR). Copies of ${ }^{1} \mathrm{H}$ and ${ }^{13} \mathrm{C}$ spectra can be found at the end of the Supporting Information. ${ }^{1} \mathrm{H}$ NMR experiments are reported in units, parts per million ( $\mathrm{ppm})$, and were measured relative to residual chloroform (7.26 ppm) in the deuterated solvent. ${ }^{13} \mathrm{C}$ NMR spectra are reported in ppm relative to deuterochloroform (77.23 ppm), and all were obtained with ${ }^{1} \mathrm{H}$ decoupling. Coupling constants were reported in Hz. FT-IR spectra were recorded on a Nicolet 6700 Thermo Scientific FT-IR spectrometer. Mass spectral data of unknown compounds were acquired at the Korea Basic Science Institute (Daegu) on a Jeol JMS 700 high-resolution mass spectrometer. A quadrupole mass analyzer was used for HRMS measurements. Melting points of all solid compounds were recorded on a Stuart SMP30 apparatus. 


\section{Controlled Experiments}

Scheme S1. E/Z-isomerization in the reaction of $1 d$ to $2 d$.

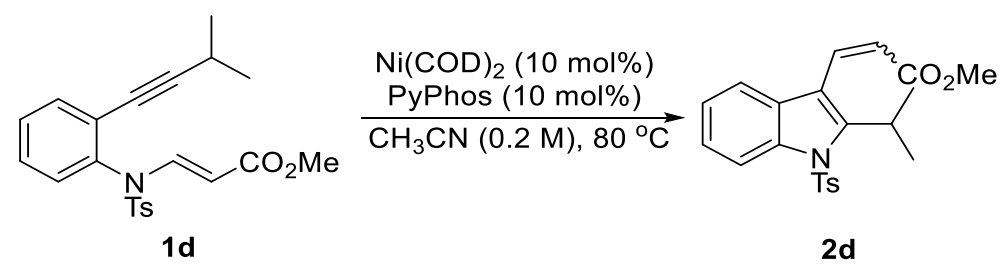

\begin{tabular}{|c|c|c|c|c|}
\hline time (h) & 1 & 2 & 8 & 12 \\
\hline $\boldsymbol{E} / \boldsymbol{Z}(\mathbf{2 d})$ & $1.3 / 1$ & $1 / 1.2$ & $1 / 2$ & $1 / 4$ \\
\hline
\end{tabular}

Scheme S2. Cross-over experiment.<smiles>CC#Cc1ccc(C(=O)OC)cc1N([13CH3])/C=C/C(C)=O</smiles> 


\section{Experimental Procedures}

\section{Synthesis of substrates (1 and 3)}
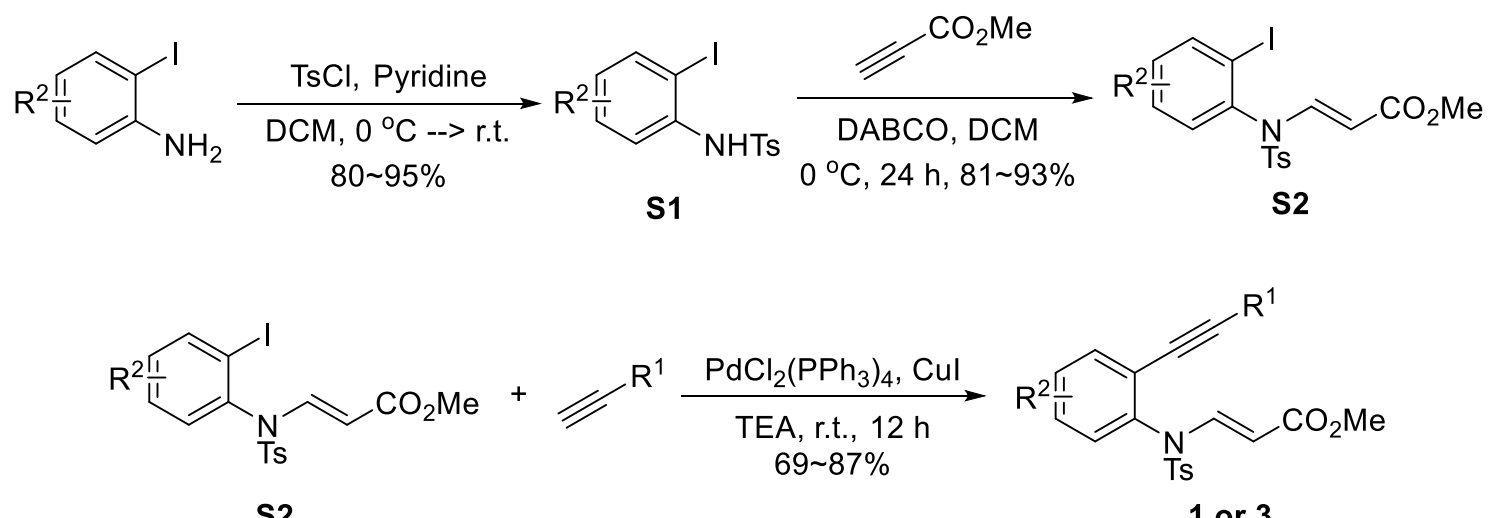

s2

1 or 3

To a stirred solution of 2-iodoaniline (1.0 equiv.) and pyridine (2.0 equiv.) in DCM was added TsCl (1.1 equiv.) at $0{ }^{\circ} \mathrm{C}$. The reaction mixture was stirred at $0{ }^{\circ} \mathrm{C}$ for $1 \mathrm{~h}$ and then allowed to warm to room temperature. After $12 \mathrm{~h}$, the reaction mixture was diluted with DCM and washed with aqueous solutions (1 $\mathrm{M}$ aqueous $\mathrm{HCl}$, saturated $\mathrm{NaHCO}_{3}$ and brine). The organic layer was separated, dried over $\mathrm{MgSO}_{4}$, and concentrated under vacuo. The resulting $N$-(2-iodophenyl)-4-methylbenzenesulfonamide derivatives S1 was utilized for next step without further purification.

DABCO (0.1 equiv.) was added to a solution of the respective $N$-(2-Iodophenyl)-4methylbenzenesulfonamides S1 (1.0 equiv.) and methyl propiolate (1.0 equiv.) in DCM (0.2 M) at $0{ }^{\circ} \mathrm{C}$. The mixture was stirred at ambient temperature for $1 \mathrm{~h}$ before the solvent was removed in vacuo. The residue was purified by flash chromatography (hexane/EtOAc) to afford methyl (E)-3-(( $N$-(2-iodophenyl)4-methylphenyl)sulfonamido)acrylates $\mathbf{S 2} *$ (unless otherwise mentioned).

To a solution of methyl 3-(( $N$-(2-iodophenyl)-4-methylphenyl)sulfonamido)acrylate $\mathbf{S 2}$ (1.0 equiv.) and alkyne (1.5 equiv.) in triethylamine $(0.5 \mathrm{M})$ was added $\mathrm{PdCl}_{2}\left(\mathrm{PPh}_{3}\right)(4.0 \mathrm{~mol} \%)$ and $\mathrm{CuI}(8 \mathrm{~mol} \%)$ under inert atmosphere. The resulting mixture was stirred at ambient temperature and the progress of the reaction was monitored by thin layer chromatography or gas chromatography. Upon completion, the reaction was quenched with water, extracted with DCM, and washed with saturated $\mathrm{NH}_{4} \mathrm{Cl}$ solution and brine. The combined organic phase was concentrated, and the residue was purified by column chromatography on silica gel using hexane/ethyl acetate to get pure methyl (E)-3-((4-methyl- $N$-(2-(alkynyl) phenyl) phenyl) sulfonamido) acrylates 1 or $3 *$.

*In general, 5-10\% of inseparable $\mathbf{Z}$ isomers are included, and substrates $\mathbf{1 c}, \mathbf{1 n}, \mathbf{1 0}, \mathbf{3 b}, \mathbf{3 f}, \mathbf{3 i}$, and $\mathbf{3 k}$ were obtained as low ratio of $E / Z$-isomers. 
trans- Carboamination of internal alkyne for the synthesis of indoles ( 2 and 4 )

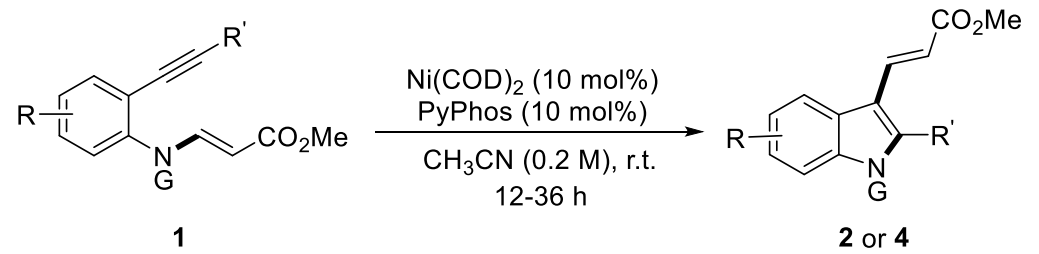

In an oven-dried $10 \mathrm{~mL}$ reaction tube, appropriate substrate $1(0.3 \mathrm{mmol})$, PyPhos (10 mol\%), Ni(COD) 2 (10 mol\%), and $\mathrm{CH}_{3} \mathrm{CN}(1.5 \mathrm{~mL})$ were mixed with constant stirring using a magnetic stir bar under argon atmosphere at ambient temperature. The reaction progress was monitored by thin layer chromatography or gas chromatography. After the reaction was completed, the mixture was concentrated under reduced pressure and purified by silica gel flash column chromatography using a hexane-ethyl acetate mixture as the eluent to give the corresponding 3-alkenyl indole product 2 or 4.

\section{Gram scale reaction of $1 \mathrm{a}$}

In an oven-dried $50 \mathrm{~mL}$ round-bottom flask, $1 \mathrm{a}(3 \mathrm{mmol}, 1.1 \mathrm{~g})$, PyPhos (10 mol\%, $0.087 \mathrm{~g}), \mathrm{Ni}(\mathrm{COD})_{2}$ (10 mol\%, $0.083 \mathrm{~g})$, and $\mathrm{CH}_{3} \mathrm{CN}(15 \mathrm{~mL})$ were mixed with constant stirring using a magnetic stir bar under argon atmosphere at ambient temperature for 24 hours. The mixture was concentrated under reduced pressure and purified by silica gel flash column chromatography using a hexane-ethyl acetate mixture as the eluent to give the 3-alkenyl indole product $\mathbf{2 a}$.

\section{Synthesis of carbazoles (8)}

Carbazoles $\mathbf{8}$ were synthesized by modified procedure from the literature. ${ }^{\mathrm{S} 2}$

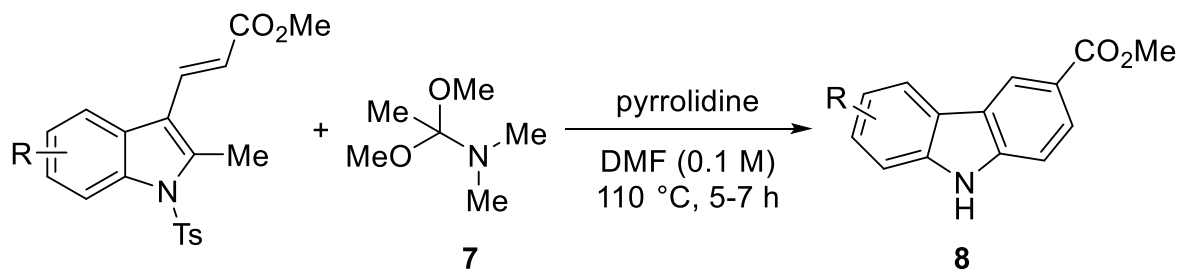

To a stirred solution of an indole $(0.2 \mathrm{mmol})$ in dry DMF $(2.0 \mathrm{~mL}), N, N$-Dimethylformamide dimethyl acetal (DMF-DMA) $7(0.4 \mathrm{mmol})$ and pyrrolidine $(0.08 \mathrm{~mL})$ were added. The reaction mixture was heated in an oil bath at $110{ }^{\circ} \mathrm{C}$ for $5-7 \mathrm{~h}$ under argon atmosphere. Reaction mixture was poured into crushed ice followed by the addition of few drops of concentrated $\mathrm{HCl}$ and extracted with $\mathrm{CHCl}_{3}$. The combined organic phase was washed with water, brine and dried over $\mathrm{MgSO}_{4}$. The mixture was concentrated under reduced 
pressure and purified by silica gel flash column chromatography using a hexane-ethyl acetate mixture as the eluent to give the corresponding carbazole 8 .

Ref S2. Sureshbabu, R.; Mohanakrishnan, A. K., An Improved Synthesis of Carbazoles via Domino Reaction of $N$-Protected-2-methylindoles with DMF-DMA/DMA-DMA. J. Heterocycl. Chem. 2012, 49, 913-918.

\section{Analytic Data for Synthesized Compounds}

\section{Analytical data for substrates (1 and 3):}

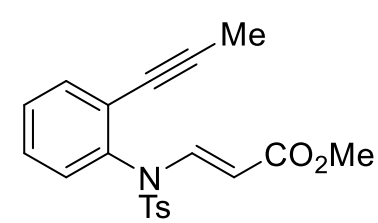

methyl(E)-3-((4-methyl- $N$-(2-(prop-1-yn-1-yl)phenyl)phenyl)sulfonamido)acr ylate, 1a; white solid (from $5 \mathrm{mmol}$ of $\mathbf{S 2}$ to $\mathbf{1 a}, 1.6 \mathrm{~g}, 85 \%$ ); melting point 105$107{ }^{\circ} \mathrm{C} ;{ }^{1} \mathbf{H}$ NMR $(\mathbf{6 0 0} \mathbf{~ M H z , C D C l}) \delta 8.28(\mathrm{~d}, J=13.7 \mathrm{~Hz}, 1 \mathrm{H}), 7.62(\mathrm{~d}, J=8.2$ $\mathrm{Hz}, 2 \mathrm{H}), 7.41(\mathrm{dd}, J=7.5,1.5 \mathrm{~Hz}, 1 \mathrm{H}), 7.35(\mathrm{td}, J=7.5,1.5 \mathrm{~Hz}, 1 \mathrm{H}), 7.31(\mathrm{td}, J$ $=7.7,1.5 \mathrm{~Hz}, 1 \mathrm{H}), 7.29(\mathrm{~d}, J=8.2 \mathrm{~Hz}, 2 \mathrm{H}), 7.04(\mathrm{dd}, J=7.7,1.5 \mathrm{~Hz}, 1 \mathrm{H}), 4.54(\mathrm{~d}, J=13.7 \mathrm{~Hz}, 1 \mathrm{H}), 3.67$ (s, 3H), 2.43 (s, 3H), 1.74 (s, 3H); ${ }^{13} \mathbf{C}$ NMR (151 MHz, $\left.\mathbf{C D C l}_{3}\right) \delta$ 168.0, 144.8, 143.1, 136.0, 135.7, 134.0, 131.8, 129.9, 129.9, 129.0, 129.3, 125.3, 99.1, 92.0, 76.4, 52.5, 21.8, 4.7; IR (neat): $v_{\max }=2950,2361$, 1712, 1622, 1364, 1167, $575 \mathrm{~cm}^{-1}$; HRMS (EI) m/z: [M+ Calcd for $\mathrm{C}_{20} \mathrm{H}_{19} \mathrm{NO}_{4} \mathrm{~S}$ 369.1035, found 369.1032; $\boldsymbol{R}_{f} 0.52(\mathrm{Hex} / \mathrm{EtOAc}, 4 / 1)$.

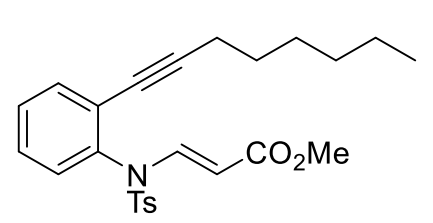

methyl(E)-3-((4-methyl- $N$-(2-(oct-1-yn-1-yl)phenyl)phenyl)sulfonamido)acr ylate, $\mathbf{1 b}$; colorless oil (from $2 \mathrm{mmol}$ of $\mathbf{S 2}$ to $\mathbf{1 b}, 690 \mathrm{mg}, 74 \%$ ); ${ }^{1} \mathbf{H}$ NMR $\left(\mathbf{6 0 0} \mathbf{M H z}, \mathbf{C D C l}_{3}\right) \delta 8.27(\mathrm{~d}, J=13.7 \mathrm{~Hz}, 1 \mathrm{H}), 7.60(\mathrm{~d}, J=7.8 \mathrm{~Hz}, 2 \mathrm{H}), 7.42$ $(\mathrm{dd}, J=7.6,1.6 \mathrm{~Hz}, 1 \mathrm{H}), 7.34(\mathrm{td}, J=7.6,1.3 \mathrm{~Hz}, 1 \mathrm{H}), 7.31-7.25(\mathrm{~m}, 1 \mathrm{H})$, $7.27(\mathrm{dd}, J=7.8,2 \mathrm{H}), 7.00(\mathrm{~d}, J=7.8 \mathrm{~Hz}, 1 \mathrm{H}), 4.55(\mathrm{~d}, J=13.7 \mathrm{~Hz}, 1 \mathrm{H}), 3.66$ (s, 3H), 2.43 (s, 3H), 2.14$2.08(\mathrm{~m}, 2 \mathrm{H}), 1.42-1.37(\mathrm{~m}, 2 \mathrm{H}), 1.34-1.28(\mathrm{~m}, 4 \mathrm{H}), 1.26-1.22(\mathrm{~m}, 2 \mathrm{H}), 0.89(\mathrm{t}, J=7.2 \mathrm{~Hz}, 3 \mathrm{H}) ;{ }^{13} \mathbf{C} \mathbf{N M R}$ (151 MHz, $\left.\mathbf{C D C l}_{3}\right) \delta 167.8,144.8,143.0,136.0,135.7,134.0,130.5,129.9,129.8,128.8,128.2,125.4$, 99.3, 96.8, 76.3, 51.4, 31.5, 28.7, 29.5, 24.3, 21.8, 18.9, 13.4; IR (neat): $v_{\max }=2929,2360,1738,1623$, 1367, 1164, 661, $576 \mathrm{~cm}^{-1}$; HRMS (EI) m/z: [M+] Calcd for $\mathrm{C}_{25} \mathrm{H}_{29} \mathrm{NO}_{4} \mathrm{~S}$ 439.181, found 439.1838; $\boldsymbol{R}_{\boldsymbol{f}}$ 0.62 (Hex/EtOAc, 4/1). 


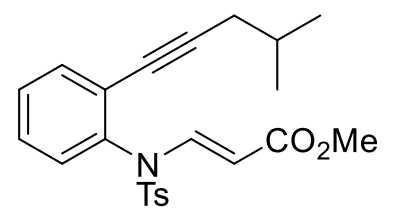

methyl(E)-3-((4-methyl- $N$-(2-(4-methylpent-1-yn-1-yl)phenyl)phenyl)sulfona mido)acrylate, 1c; colorless oil (from $2 \mathrm{mmol}$ of $\mathbf{S 2}$ to $\mathbf{1 c}, 610 \mathrm{mg}, 74 \%$ ); ${ }^{1} \mathbf{H}$ NMR (600 MHz, $\left.\mathbf{C D C l}_{3}\right) \delta 8.28(\mathrm{~d}, J=13.8 \mathrm{~Hz}, 1 \mathrm{H}), 7.61(\mathrm{~d}, J=8.2 \mathrm{~Hz}, 2 \mathrm{H})$, $7.44(\mathrm{~d}, J=7.6 \mathrm{~Hz}, 1 \mathrm{H}), 7.35$ (t, $J=7.6 \mathrm{~Hz}, 1 \mathrm{H}), 7.30(\mathrm{t}, J=7.9 \mathrm{~Hz}, 1 \mathrm{H}), 7.28$ (d, $J=8.2 \mathrm{~Hz}, 2 \mathrm{H}), 6.99(\mathrm{~d}, J=7.9 \mathrm{~Hz}, 1 \mathrm{H}), 4.56(\mathrm{~d}, J=13.8 \mathrm{~Hz}, 1 \mathrm{H}), 3.66(\mathrm{~s}, 3 \mathrm{H}), 2.43(\mathrm{~s}, 3 \mathrm{H}), 2.01(\mathrm{dd}$, $J=6.7,2.2 \mathrm{~Hz}, 2 \mathrm{H}), 1.72(\mathrm{tq}, J=6.6,6.7 \mathrm{~Hz}, 1 \mathrm{H}), 0.92(\mathrm{~d}, J=6.6 \mathrm{~Hz}, 6 \mathrm{H}) ;{ }^{13} \mathbf{C} \mathbf{~ N M R}\left(\mathbf{1 5 1} \mathbf{~ M H z}, \mathbf{C D C l}_{3}\right)$ $\delta$ 167.8, 144.8, 143.0, 135.9, 135.6, 134.1, 130.5, 130.0, 129.8, 128.8, 128.3, 125.5, 99.5, 95.8, 77.1, 51.4, 28.9, 28.2, 23.2, 22.9; IR (neat): $v_{\max }=2955,2360,1716,1624,1367,1168,577 \mathrm{~cm}^{-1}$; HRMS (EI) m/z: $\left[\mathrm{M}^{+}\right]$Calcd for $\mathrm{C}_{23} \mathrm{H}_{25} \mathrm{NO}_{4} \mathrm{~S}$ 411.1504, found 411.1508; $\boldsymbol{R}_{f} 0.55$ (Hex/EtOAc, 4/1).

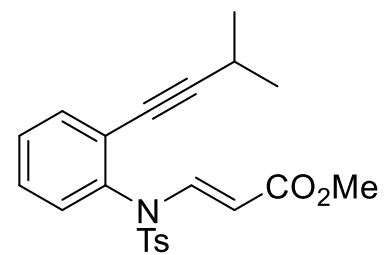

methyl(E)-3-((4-methyl- $N$-(2-(3-methylbut-1-yn-1-yl)phenyl)phenyl)sulfonam ido)acrylate, 1d; brown solid (from $2 \mathrm{mmol}$ of $\mathbf{S 2}$ to $\mathbf{1 d}, 564 \mathrm{mg}, 71 \%$ ); melting point $78-80{ }^{\circ} \mathrm{C} ;{ }^{1} \mathbf{H}$ NMR $\left(\mathbf{6 0 0} \mathbf{~ M H z}, \mathbf{C D C l}_{\mathbf{3}}\right) \delta 8.26(\mathrm{~d}, J=13.8 \mathrm{~Hz}, 1 \mathrm{H}), 7.59$ (d, $J=8.3 \mathrm{~Hz}, 2 \mathrm{H}), 7.42(\mathrm{dd}, J=7.6,1.4 \mathrm{~Hz}, 1 \mathrm{H}), 7.34(\mathrm{td}, J=7.6,1.4 \mathrm{~Hz}, 1 \mathrm{H})$, 7.30 (dd, $J=8.0,1.6 \mathrm{~Hz}, 1 \mathrm{H}), 7.28(\mathrm{~d}, J=8.0 \mathrm{~Hz}, 2 \mathrm{H}), 7.04-6.99(\mathrm{~m}, 1 \mathrm{H}), 4.58(\mathrm{~d}, J=13.8 \mathrm{~Hz}, 1 \mathrm{H}), 3.67$ (s, 3H), $2.48(\mathrm{tt}, J=6.9 \mathrm{~Hz}, 1 \mathrm{H}), 2.43$ (s, 3H), 1.07 (dd, $J=9.7,6.9 \mathrm{~Hz}, 6 \mathrm{H}) ;{ }^{13} \mathbf{C} \mathbf{N M R}\left(\mathbf{1 5 1} \mathbf{~ M H z}, \mathbf{C D C l}_{3}\right)$ $\delta 168.9,144.8,143.8,136.1,135.7,133.8,130.5,129.0,129.8,128.8,128.2,125.3,102.3,99.5,75.7,51.4$, 23.5, 23.7, 21.8, 21.4; IR (neat): $v_{\max }=2972,2331,1711,1623,1369,1169,1085,734,577 \mathrm{~cm}^{-1} ;$ HRMS (EI) $\mathrm{m} / \mathrm{z}:\left[\mathrm{M}^{+}\right]$Calcd for $\mathrm{C}_{22} \mathrm{H}_{23} \mathrm{NO}_{4} \mathrm{~S}$ 397.1348, found 397.1345; $\boldsymbol{R}_{\boldsymbol{f}} 0.55$ (Hex/EtOAc, 4/1).

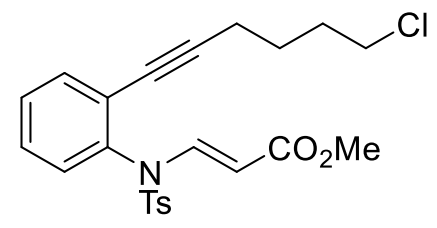

methyl(E)-3-((N-(2-(6-chlorohex-1-yn-1-yl)phenyl)-4-methylphenyl)sulfon amido)acrylate, 1e; colorless oil (from $2 \mathrm{mmol}$ of $\mathbf{S 2}$ to $\mathbf{1 e}, 664 \mathrm{mg}, 70 \%$ ); ${ }^{1} \mathbf{H}$ NMR (600 MHz, CDCl $) \delta 8.27(\mathrm{~d}, J=13.8 \mathrm{~Hz}, 1 \mathrm{H}), 7.62(\mathrm{~d}, J=8.1 \mathrm{~Hz}$, 2H), 7.45 (dd, $J=7.6,1.4 \mathrm{~Hz}, 1 \mathrm{H}), 7.36(\mathrm{td}, J=7.6,1.4 \mathrm{~Hz}, 1 \mathrm{H}), 7.32$ (td, $J$ $=7.8,1.3 \mathrm{~Hz}, 1 \mathrm{H}), 7.30(\mathrm{~d}, J=8.1 \mathrm{~Hz}, 2 \mathrm{H}) 6.94(\mathrm{dd}, J=7.8,1.3 \mathrm{~Hz}, 1 \mathrm{H}), 4.55(\mathrm{~d}, J=13.8 \mathrm{~Hz}, 1 \mathrm{H}), 3.68$ (s, 3H), 3.55 ((t, $J=6.5 \mathrm{~Hz}, 2 \mathrm{H}), 2.44(\mathrm{~s}, 3 \mathrm{H}), 2.24-2.19(\mathrm{~m}, 2 \mathrm{H}), 1.88-1.81(\mathrm{~m}, 2 \mathrm{H}), 1.65-1.57(\mathrm{~m}, 2 \mathrm{H})$; ${ }^{13} \mathbf{C}$ NMR (151 MHz, $\left.\mathbf{C D C l}_{3}\right) \delta$ 167.8, 144.0, 143.1, 136.2, 135.8, 134.0, 130.3, 130.0, 129.9, 129.0, 128.2, 125.3, 122.0, 99.5, 95.6, 51.5, 44.8, 31.5, 25.2, 21.9, 18.9; IR (neat): $v_{\max }=2950,2361,1710,1622,1368$, $1167,654,575 \mathrm{~cm}^{-1} ;$ HRMS (EI) m/z: $\left[\mathrm{M}^{+}\right]$Calcd for $\mathrm{C}_{23} \mathrm{H}_{24} \mathrm{ClNO}_{4} \mathrm{~S} 445.1115$, found $445.1112 ; \boldsymbol{R}_{\boldsymbol{f}} 0.55$ (Hex/EtOAc, 4/1). 


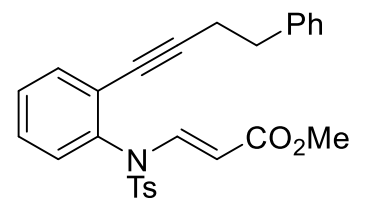

methyl(E)-3-((4-methyl- $N$-(2-(4-phenylbut-1-yn-1-yl)phenyl)phenyl)sulfonami do)acrylate, 1f; colorless oil (from $2 \mathrm{mmol}$ of $\mathbf{S 2}$ to $\mathbf{1 f}, 726 \mathrm{mg}, 79 \%$ ); ${ }^{1} \mathbf{H}$ NMR $\left(\mathbf{6 0 0} \mathbf{M H z}, \mathbf{C D C l}_{3}\right) \delta 8.30(\mathrm{~d}, J=13.7 \mathrm{~Hz}, 1 \mathrm{H}), 7.57(\mathrm{~d}, J=8.0 \mathrm{~Hz}, 2 \mathrm{H}), 7.41(\mathrm{dd}$, $J=7.7,1.6 \mathrm{~Hz}, 1 \mathrm{H}), 7.35(\mathrm{td}, J=7.6,1.5 \mathrm{~Hz}, 1 \mathrm{H}), 7.31(\mathrm{t}, J=7.6 \mathrm{~Hz}, 1 \mathrm{H}), 7.30$ $(\mathrm{d}, J=8.0 \mathrm{~Hz}, 2 \mathrm{H}), 7.24-7.19(\mathrm{~m}, 3 \mathrm{H}), 7.19(\mathrm{~d}, J=6.7 \mathrm{~Hz}, 2 \mathrm{H}), 7.02(\mathrm{dd}, J=7.7,1.6 \mathrm{~Hz}, 1 \mathrm{H}), 4.56(\mathrm{~d}, J=$ $13.7 \mathrm{~Hz}, 1 \mathrm{H}), 3.68(\mathrm{~s}, 3 \mathrm{H}), 2.73(\mathrm{t}, J=7.5 \mathrm{~Hz}, 2 \mathrm{H}), 2.43(\mathrm{t}, J=7.5 \mathrm{~Hz}, 2 \mathrm{H}), 2.41(\mathrm{~s}, 3 \mathrm{H}) ;{ }^{13} \mathbf{C} \mathbf{N M R}(\mathbf{1 5 1}$ MHz, $\left.\mathbf{C D C l}_{3}\right) \delta 167.7,144.6,142.0,140.5,135.8,135.5,133.9,130.4,129.7,129.7,128.8,128.4,128.4$, 127.5, 126.4, 125.1, 99.0, 95.5, 76.7, 51.3, 34.7, 21.7, 21.6; IR (neat): $v_{\max }=2970,2360,1718,1623,1367$, 1169, $577 \mathrm{~cm}^{-1}$; HRMS (EI) m/z: [M+] Calcd for $\mathrm{C}_{27} \mathrm{H}_{25} \mathrm{NO}_{4} \mathrm{~S}$ 459.1504, found 459.1503; $\boldsymbol{R}_{\boldsymbol{f}} 0.52$ (Hex/EtOAc, 4/1).

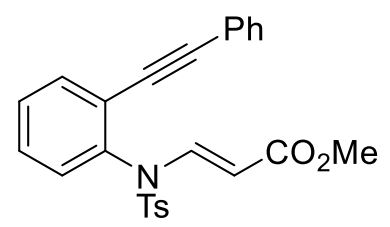

methyl(E)-3-((4-methyl- $N$-(2-(phenylethynyl)phenyl)phenyl)sulfonamido)acr ylate, 1g; white solid (from $2 \mathrm{mmol}$ of $\mathbf{S 2}$ to $\mathbf{1 g}, 750 \mathrm{mg}, 87 \%$ ); melting point $82-84{ }^{\circ} \mathrm{C} ;{ }^{1} \mathbf{H}$ NMR $\left(\mathbf{6 0 0} \mathbf{M H z}, \mathbf{C D C l}_{3}\right) \delta 8.35(\mathrm{~d}, J=13.7 \mathrm{~Hz}, 1 \mathrm{H}), 7.59(\mathrm{~d}, J=$ $8.2 \mathrm{~Hz}, 2 \mathrm{H}), 7.55(\mathrm{dd}, J=7.4,1.8 \mathrm{~Hz}, 1 \mathrm{H}), 7.44(\mathrm{td}, J=7.3,1.5 \mathrm{~Hz}, 1 \mathrm{H}), 7.43$ 7.40 (m, 1H), 7.43-7.28 (m, 1H), $7.30(\mathrm{~d}, J=7.5 \mathrm{~Hz}, 2 \mathrm{H}), 7.25$ (d, $J=8.2 \mathrm{~Hz}, 2 \mathrm{H}), 7.20$ (dd, $J=7.4,1.8$ $\mathrm{Hz}, 1 \mathrm{H}), 7.09$ (d, $J=7.5 \mathrm{~Hz}, 2 \mathrm{H}), 4.66(\mathrm{~d}, J=13.7 \mathrm{~Hz}, 1 \mathrm{H}), 3.67(\mathrm{~s}, 3 \mathrm{H}), 2.23(\mathrm{~s}, 3 \mathrm{H}) ;{ }^{13} \mathbf{C} \mathbf{N M R}(\mathbf{1 5 1}$ MHz, $\left.\mathbf{C D C l}_{3}\right) \delta 168.7,144.0,142.9,136.2,135.5,133.8,131.8,131.2,130.0,130.0,129.7,128.7,128.4$, 128.1, 124.4, 122.8, 99.7, 96.8, 85.1, 50.5, 21.7; IR (neat): $v_{\max }=2970,2360,1719,1625,1367,1170,578$ $\mathrm{cm}^{-1}$; HRMS (EI) m/z: [M+] Calcd for $\mathrm{C}_{25} \mathrm{H}_{21} \mathrm{NO}_{4} \mathrm{~S} 431.1191$, found 431.1193; $\boldsymbol{R}_{f} 0.51$ (Hex/EtOAc, 4/1).

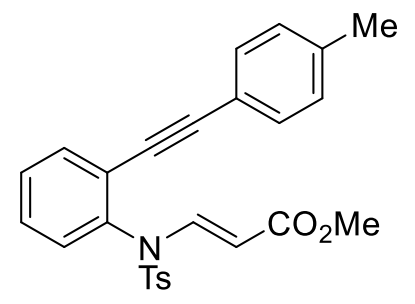

methyl(E)-3-((4-methyl- $N$-(2-(p-tolylethynyl)phenyl)phenyl)sulfonamido)acr ylate, 1h; white solid (from $2 \mathrm{mmol}$ of $\mathbf{S 2}$ to $\mathbf{1 h}, 720 \mathrm{mg}, 81 \%$ ); melting point 137-139 ${ }^{\circ} \mathrm{C} ;{ }^{1} \mathbf{H}$ NMR $\left(\mathbf{6 0 0} \mathbf{~ M H z}, \mathbf{C D C l}_{3}\right) \delta 8.34(\mathrm{~d}, J=13.8 \mathrm{~Hz}, 1 \mathrm{H}), 7.58(\mathrm{~d}$, $J=8.2 \mathrm{~Hz}, 2 \mathrm{H}), 7.53(\mathrm{dd}, J=7.5,1.7 \mathrm{~Hz}, 1 \mathrm{H}), 7.40-7.35$ (m, 2H), 7.19 (dd, $J$ = 7.5, $1.7 \mathrm{~Hz}, 1 \mathrm{H}), 7.14(\mathrm{~d}, J=8.2 \mathrm{~Hz}, 2 \mathrm{H}), 7.12-7.07(\mathrm{~m}, 4 \mathrm{H}), 4.66(\mathrm{~d}, J=$ $13.8 \mathrm{~Hz}, 1 \mathrm{H}), 3.67$ (s, 3H), 2.36 (s, 3H), 2.24 (s, 3H); ${ }^{13} \mathbf{C}$ NMR (151 MHz, CDCl $\left.\mathbf{3}\right) \delta$ 167.9, 144.9, 142.0, 139.0, 136.0, 135.5, 133.7, 131.7, 131.2, 130.1, 129.9, 129.5, 129.1, 128.1, 124.6, 119.8, 99.6, 95.4, 84.5, 51.5, 21.8, 21.7; IR (neat): $v_{\max }=2949,2360,1715,1623,1366,1168,575 \mathrm{~cm}^{-1} ; \mathbf{H R M S}(\mathrm{EI}) \mathrm{m} / \mathrm{z}:\left[\mathrm{M}^{+}\right]$ Calcd for $\mathrm{C}_{26} \mathrm{H}_{23} \mathrm{NO}_{4} \mathrm{~S} 445.1348$, found 445.1350; $\boldsymbol{R}_{f} 0.51$ (Hex/EtOAc, 4/1). 


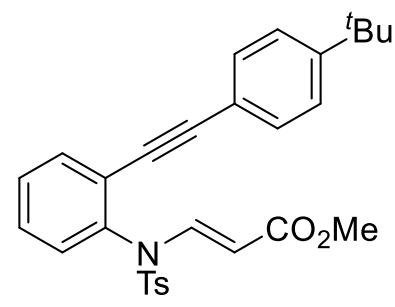

methyl $(E)-3-((N-(2-((4-$ (tert-butyl)phenyl)ethynyl)phenyl)-4-methylphenyl)su Ifonamido)acrylate, 1i; white solid (from $2 \mathrm{mmol}$ of $\mathbf{S 2}$ to $\mathbf{1 i}, 840 \mathrm{mg}, 86 \%$ ); melting point $138-140{ }^{\circ} \mathrm{C} ;{ }^{1} \mathbf{H} \mathbf{~ N M R}\left(\mathbf{6 0 0} \mathbf{~ M H z}, \mathbf{C D C l}_{3}\right) \delta 8.34(\mathrm{~d}, J=13.7 \mathrm{~Hz}$, $1 \mathrm{H}), 7.59(\mathrm{~d}, J=8.3 \mathrm{~Hz}, 2 \mathrm{H}), 7.54(\mathrm{dd}, J=7.5,1.9 \mathrm{~Hz}, 1 \mathrm{H}), 7.40$ ( td, $J=7.5$, $1.5 \mathrm{~Hz}, 1 \mathrm{H}), 7.44-7.36(\mathrm{~m}, 1 \mathrm{H}), 7.32(\mathrm{~d}, J=8.4 \mathrm{~Hz}, 2 \mathrm{H}), 7.20(\mathrm{~d}, J=8.4 \mathrm{~Hz}$, 2H), $7.18(\mathrm{dd}, J=7.5,1.5 \mathrm{~Hz}, 1 \mathrm{H}), 7.08(\mathrm{~d}, J=8.3 \mathrm{~Hz}, 2 \mathrm{H}), 4.66(\mathrm{~d}, J=13.7 \mathrm{~Hz}, 1 \mathrm{H}), 3.67(\mathrm{~s}, 3 \mathrm{H}), 2.22$ (s, 3H), 1.32 (s, 9H); ${ }^{13} \mathbf{C}$ NMR (151 MHz, CDCl 3 ) $\delta$ 167.8, 152.2, 144.9, 142.9, 136.1, 135.5, 133.7, 131.6, 131.1, 130.0, 129.9, 129.4, 128.1, 125.3, 124.5, 119.7, 99.6, 95.4, 83.9, 50.3, 35.6, 31.0, 21.7; IR (neat): $v_{\max }=2970,2361,1717,1624,1369,1131,578 \mathrm{~cm}^{-1}$; HRMS (EI) m/z: [M+ $\mathrm{M}^{+}$Calcd for $\mathrm{C}_{29} \mathrm{H}_{29} \mathrm{NO}_{4} \mathrm{~S}$ 487.1817, found 487.1819; $\boldsymbol{R}_{f} 0.53$ (Hex/EtOAc, 4/1).

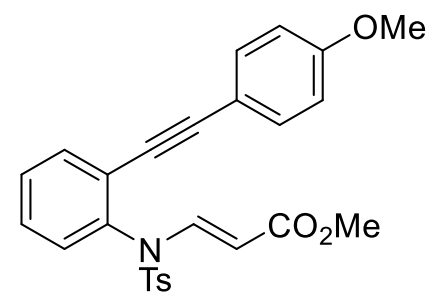

methyl(E)-3-((N-(2-((4-methoxyphenyl)ethynyl)phenyl)-4-methylphenyl)sul fonamido)acrylate, $\mathbf{1 j}$; white solid (from $2 \mathrm{mmol}$ of $\mathbf{S 2}$ to $\mathbf{1 j}$, $766 \mathrm{mg}, 83 \%$ ); melting point $136-138{ }^{\circ} \mathrm{C} ;{ }^{1} \mathbf{H}$ NMR (600 $\left.\mathbf{~ M H z , ~} \mathbf{C D C l}_{3}\right) \delta 8.34(\mathrm{~d}, J=13.7$ $\mathrm{Hz}, 1 \mathrm{H}), 7.59(\mathrm{~d}, J=8.1 \mathrm{~Hz}, 2 \mathrm{H}), 7.52(\mathrm{dd}, J=7.7,1.6 \mathrm{~Hz}, 1 \mathrm{H}), 7.41(\mathrm{td}, J$ = 7.6, $1.5 \mathrm{~Hz}, 1 \mathrm{H}), 7.37(\mathrm{td}, J=7.6,1.5 \mathrm{~Hz}, 1 \mathrm{H}), 7.19(\mathrm{~d}, J=8.9 \mathrm{~Hz}, 2 \mathrm{H})$, $7.17(\mathrm{dd}, J=7.7,1.6 \mathrm{~Hz}, 1 \mathrm{H}), 7.10(\mathrm{~d}, J=7.8 \mathrm{~Hz}, 2 \mathrm{H}), 6.83(\mathrm{~d}, J=8.9 \mathrm{~Hz}, 2 \mathrm{H}), 4.66(\mathrm{~d}, J=13.8 \mathrm{~Hz}, 1 \mathrm{H})$, 3.83 (s, 3H), 3.67 (s, 3H), 2.26 (s, 3H); ${ }^{13} \mathbf{C}$ NMR (151 MHz, CDCl 3 ) $\delta$ 167.9, 160.1, 144.9, 142.0, 135.9, 135.4, 133.5, 133.4, 131.1, 130.0, 129.0, 129.3, 128.1, 124.8, 115.0, 114.1, 99.6, 95.4, 83.0, 55.6, 51.5, 21.8; IR (neat): $v_{\max }=2970,2361,1719,1624,1367,1331,578 \mathrm{~cm}^{-1}$; HRMS (EI) m/z: [M+] Calcd for $\mathrm{C}_{26} \mathrm{H}_{23} \mathrm{NO}_{5} \mathrm{~S}$ 461.1297, found 461.1295; $\boldsymbol{R}_{\boldsymbol{f}} 0.50$ (Hex/EtOAc, 4/1).

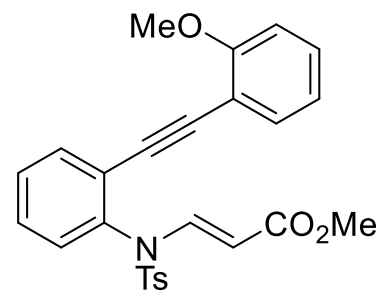

methyl(E)-3-((N-(2-((2-methoxyphenyl)ethynyl)phenyl)-4-methylphenyl)sulfo namido)acrylate, 1k; white solid (from $2 \mathrm{mmol}$ of $\mathbf{S 2}$ to $\mathbf{1 k}, 710 \mathrm{mg}, 77 \%$ ); melting point $86-88{ }^{\circ} \mathrm{C} ;{ }^{1} \mathbf{H}$ NMR $\left(600 \mathrm{MHz}, \mathbf{l}_{3}\right) \boldsymbol{\delta} 8.37(\mathrm{~d}, J=13.7 \mathrm{~Hz}, 1 \mathrm{H})$, $7.58(\mathrm{~d}, J=8.2 \mathrm{~Hz}, 2 \mathrm{H}), 7.55(\mathrm{dd}, J=7.5,1.9 \mathrm{~Hz}, 1 \mathrm{H}), 7.42-7.36(\mathrm{~m}, 2 \mathrm{H}), 7.29$ (ddd, $J=8.3,7.6,1.7 \mathrm{~Hz}, 1 \mathrm{H}), 7.22(\mathrm{dd}, J=7.5,1.7 \mathrm{~Hz}, 1 \mathrm{H}), 7.17(\mathrm{dd}, J=7.6$, $1.7 \mathrm{~Hz}, 1 \mathrm{H}), 7.01(\mathrm{~d}, J=8.2 \mathrm{~Hz}, 2 \mathrm{H}), 6.87(\mathrm{t}, J=7.6 \mathrm{~Hz}, 1 \mathrm{H}), 6.84(\mathrm{~d}, J=8.3 \mathrm{~Hz}, 1 \mathrm{H}), 4.64(\mathrm{~d}, J=13.7$ Hz, 1H), 3.88 (s, 3H), 3.66 (s, 3H), 2.18 (s, 3H); ${ }^{13} \mathbf{C ~ N M R ~ ( 1 5 1 ~ M H z , ~ C D C l ~}$ N $_{\text {) }} \delta$ 169.5, 161.2, 144.8, 143.2, 135.9, 135.4, 133.7, 133.6, 131.3, 130.4, 129.9, 129.8, 129.4, 128.2, 124.7, 119.7, 112.2, 110.7, 99.2, 91.8, 89.3, 54.0, 49.5, 21.7; IR (neat): $v_{\max }=2970,2361,1718,1623,1368,1132,577 \mathrm{~cm}^{-1}$; HRMS (EI) m/z: $\left[\mathrm{M}^{+}\right]$Calcd for $\mathrm{C}_{26} \mathrm{H}_{23} \mathrm{NO}_{5} 461.1297$, found 461.1299; $\boldsymbol{R}_{f} 0.50$ (Hex/EtOAc, 4/1). 


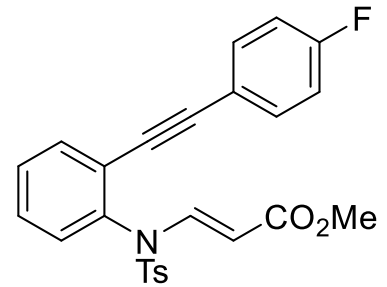

methyl(E)-3-((N-(2-((4-fluorophenyl)ethynyl)phenyl)-4-methylphenyl)sulfona mido)acrylate, 1l; white solid (from $2 \mathrm{mmol}$ of $\mathbf{S 2}$ to $\mathbf{1 1}, 737 \mathrm{mg}, 82 \%$ ); melting point $116-118{ }^{\circ} \mathrm{C} ;{ }^{1} \mathbf{H} \mathbf{~ N M R}\left(\mathbf{6 0 0} \mathbf{~ M H z}, \mathbf{C D C l}_{3}\right) \delta 8.33(\mathrm{~d}, J=13.8 \mathrm{~Hz}, 1 \mathrm{H}), 7.60$ (d, $J=8.3 \mathrm{~Hz}, 2 \mathrm{H}), 7.55$ (dd, $J=7.6,1.7 \mathrm{~Hz}, 1 \mathrm{H}), 7.43$ (ddd, $J=7.6,7.6,1.5$ $\mathrm{Hz}, 1 \mathrm{H}), 7.39(\mathrm{ddd}, J=7.7,7.6,1.7 \mathrm{~Hz}, 1 \mathrm{H}), 7.26\left(\mathrm{dd}, J=8.9 \mathrm{~Hz},{ }^{4} J_{H-F}=5.4 \mathrm{~Hz}\right.$, 2H), 7.15-7.10 (m, 3H), $7.00\left(\mathrm{dd}, J=8.9,{ }^{2} J_{H-F}=8.7 \mathrm{~Hz}, 2 \mathrm{H}\right), 4.65(\mathrm{~d}, J=13.8 \mathrm{~Hz}, 1 \mathrm{H}), 3.67(\mathrm{~s}, 3 \mathrm{H}), 2.27$ (s, 3H); ${ }^{13} \mathbf{C}$ NMR (151 MHz, CDCl $) \delta 167.8,162.9$ (d, $J=250.4$ Hz), 144.0, 142.0, 136.2, 135.5, 133.8, 133.7, 133.7, 131.0, 130.1, 130.0, 129.8, 128.1, 127.5, 124.4, 118.9 (d, $J=3.4 \mathrm{~Hz}), 115.8$ (d, $J=22.0 \mathrm{~Hz})$, 99.7, 94.0, 84.9 (d, $J=1.4 \mathrm{~Hz}), 51.5,21.7$; IR (neat): $v_{\max }=2950,2361,1713,1625,1371,1132,973,578$ $\mathrm{cm}^{-1}$; HRMS (EI) m/z: $\left[\mathrm{M}^{+}\right]$Calcd for $\mathrm{C}_{25} \mathrm{H}_{20} \mathrm{FNO}_{4} \mathrm{~S}$ 449.1097, found 449.1100; $\boldsymbol{R}_{f}$ 0.51 (Hex/EtOAc, 4/1).

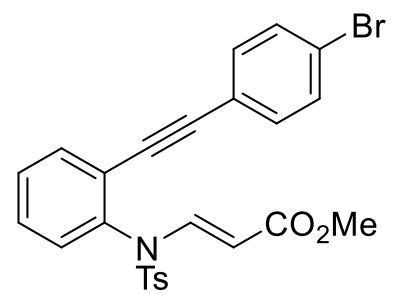

methyl(E)-3-((N-(2-((4-bromophenyl)ethynyl)phenyl)-4-methylphenyl)sulfona mido)acrylate, 1m; brown solid (from $2 \mathrm{mmol}$ of $\mathbf{S 2}$ to $\mathbf{1 m}, 880 \mathrm{mg}, 86 \%$ ); melting point $142-144{ }^{\circ} \mathrm{C} ;{ }^{1} \mathbf{H}$ NMR $\left(600 \mathbf{~ M H z}, \mathbf{C D C l}_{3}\right) \delta 8.32(\mathrm{~d}, J=13.8 \mathrm{~Hz}$, $1 \mathrm{H}), 7.59(\mathrm{~d}, J=8.4 \mathrm{~Hz}, 2 \mathrm{H}), 7.55(\mathrm{dd}, J=7.6,1.8 \mathrm{~Hz}, 1 \mathrm{H}), 7.45-7.38(\mathrm{~m}, 4 \mathrm{H})$, 7.16-7.11 (m, 5H), $4.64(\mathrm{~d}, J=13.8 \mathrm{~Hz}, 1 \mathrm{H}), 3.67(\mathrm{~s}, 3 \mathrm{H}), 2.27(\mathrm{~s}, 3 \mathrm{H}) ;{ }^{13} \mathrm{C}$ NMR (151 MHz, $\left.\mathbf{C D C l}_{3}\right) \delta 167.8,145.0,142.9,136.3,135.4,133.7,133.2,131.7,131.0,130.1,130.1$, 129.0, 128.1, 124.5, 123.2, 121.7, 99.7, 93.94, 86.2, 51.5, 21.7; IR (neat): $v_{\max }=2950,2361,1710,1624$, 1168, 734, $574 \mathrm{~cm}^{-1}$; HRMS (EI) m/z: [M+] Calcd for $\mathrm{C}_{25} \mathrm{H}_{20} \mathrm{BrNO}_{4} \mathrm{~S} 509.0296$, found 509.0299; $\boldsymbol{R}_{f} 0.52$ (Hex/EtOAc, 4/1).

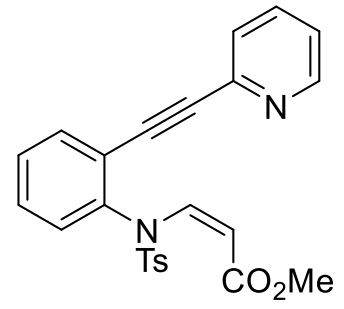

methyl(Z)-3-((4-methyl- $N$-(2-(pyridin-2-ylethynyl)phenyl)phenyl)sulfonamido)ac rylate, 1n; yellow solid (from $2 \mathrm{mmol}$ of $\mathbf{S 2}$ to $\mathbf{1 n}, 623 \mathrm{mg}, 72 \%$ ); melting point $104-106{ }^{\circ} \mathrm{C} ;{ }^{1} \mathbf{H}$ NMR $\left(600 \mathrm{MHz}, \mathbf{C D C l}_{3}\right) \delta 8.59(\mathrm{~d}, J=5.0 \mathrm{~Hz}, 1 \mathrm{H}), 7.65(\mathrm{td}, J=$ 7.9, 1.7 Hz, 1H), $7.54(\mathrm{~d}, J=7.9 \mathrm{~Hz}, 1 \mathrm{H}), 7.49(\mathrm{~d}, J=8.2 \mathrm{~Hz}, 2 \mathrm{H}), 7.49-7.45(\mathrm{~m}$, 1H), $7.05(\mathrm{~d}, J=8.2 \mathrm{~Hz}, 2 \mathrm{H}), 5.17(\mathrm{~d}, J=10.4 \mathrm{~Hz}, 1 \mathrm{H}), 3.20(\mathrm{~s}, 3 \mathrm{H}), 2.23(\mathrm{~s}, 3 \mathrm{H}) ;{ }^{13} \mathbf{C}$ NMR (151 MHz, $\left.\mathbf{C D C l}_{3}\right) \delta 164.6,150.1,145.1,143.0,139.5,136.4,136.1,134.5,133.4,132.6,130.2,129.9,128.8,128.2$, 127.3, 123.1, 121.6, 100.9, 94.2, 85.8, 51.2, 21.7; IR (neat): $v_{\max }=2970,2361,1724,1628,1365,1168$, $578 \mathrm{~cm}^{-1}$; HRMS (EI) m/z: $\left[\mathrm{M}^{+}\right]$Calcd for $\mathrm{C}_{24} \mathrm{H}_{20} \mathrm{~N}_{2} \mathrm{O}_{4} \mathrm{~S} 432.1144$, found 432.1143; $\boldsymbol{R}_{f} 0.52$ (Hex/EtOAc, $7 / 3)$. 


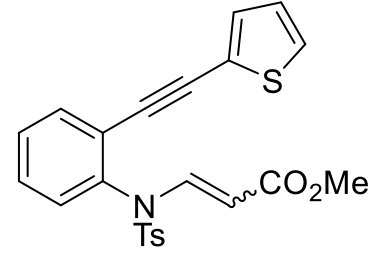

$(\mathrm{d}, J=10.3 \mathrm{~Hz}, 1 \mathrm{H}), 7.12(\mathrm{~d}, J=8.0 \mathrm{~Hz}, 2 \mathrm{H}), 7.08(\mathrm{~d}, J=8.0 \mathrm{~Hz}, 2 \mathrm{H}), 7.06-7.02(\mathrm{~m}, 2 \mathrm{H}), 6.97$ (ddd, $J=6.5,5.1,3.6$ Hz, 2H), $5.16(\mathrm{~d}, J=10.3 \mathrm{~Hz}, 1 \mathrm{H}), 4.65(\mathrm{~d}, J=13.8 \mathrm{~Hz}, 1 \mathrm{H}), 3.67(\mathrm{~s}, 3 \mathrm{H}), 3.21(\mathrm{~s}, 3 \mathrm{H}), 2.28(\mathrm{~s}, 6 \mathrm{H}) ;{ }^{13} \mathbf{C ~ N M R}(\mathbf{1 5 1}$ MHz, CDCl $\left.\mathbf{3}_{3}\right) E / Z$ mixtures $\delta$ 167.7, 164.5, 145.0, 144.9, 142.8, 138.8,136.4, 135.8, 135.1, 133.4, 132.9, 132.7, 132.4, 132.1, 131.4, 130.2, 130.0, 129.9, 129.8, 129.7, 128.7, 128.6, 128.1, 128.1, 128.0, 127.8, 127.2, 127.2, 123.9, 122.0, 122.7, 121.7, 100.9, 99.6, 89.7, 88.8, 88.7, 88.4, 51.4, 51.1, 21.8, 21.7; IR (neat): $v_{\max }=2970,2360,1720,1625,1366,1170,577 \mathrm{~cm}^{-1} ;$ HRMS (EI) m/z: [M+ Calcd for $\mathrm{C}_{23} \mathrm{H}_{19} \mathrm{NO}_{4} \mathrm{~S}_{2}$ 437.0755, found 437.0753; $\boldsymbol{R}_{f} 0.52(\mathrm{Hex} / \mathrm{EtOAc}, 7 / 3)$.

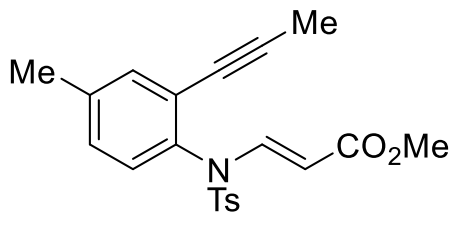

methyl(E)-3-((4-methyl- $N$-(4-methyl-2-(prop-1-yn-1-yl)phenyl)phenyl)su lfonamido)acrylate, 3a; viscous oil (from $5 \mathrm{mmol}$ of 4-methyl-2iodoaniline to 3a, $1.1 \mathrm{~g}, 56 \%) ;{ }^{1} \mathbf{H}$ NMR $\left(600 \mathbf{~ M H z}, \mathbf{C D C l}_{3}\right) \delta 8.32(\mathrm{~d}, J=$ $13.7 \mathrm{~Hz}, 1 \mathrm{H}), 7.65(\mathrm{~d}, J=8.2 \mathrm{~Hz}, 2 \mathrm{H}), 7.32(\mathrm{~d}, J=8.2 \mathrm{~Hz}, 2 \mathrm{H}), 7.26(\mathrm{~s}$, 1H), $7.14(\mathrm{~d}, J=8.1 \mathrm{~Hz}, 1 \mathrm{H}), 6.94(\mathrm{~d}, J=8.1 \mathrm{~Hz}, 1 \mathrm{H}), 4.59$ (d, $J=13.7 \mathrm{~Hz}, 1 \mathrm{H}), 3.70(\mathrm{~s}, 3 \mathrm{H}), 2.47$ (s, 3H), 2.36 (s, 3H), 1.76 (s, 3H); ${ }^{13} \mathbf{C}$ NMR (151 MHz, CDCl $) \delta$ 167.9, 144.7, 143.2, 140.1, 135.7, 134.5, 133.2, $130.4,129.9,129.8,128.2,124.8,99.1,91.3,75.4,51.3,21.7,21.1,4.6$; IR (neat): $v_{\max }=2970,2360,1715$, 1620, 1366, 1165, $565 \mathrm{~cm}^{-1}$; HRMS (EI) m/z: [ $\mathrm{M}^{+}$] Calcd for $\mathrm{C}_{21} \mathrm{H}_{21} \mathrm{NO}_{4} \mathrm{~S}$ 383.1191, found 383.1194; $\boldsymbol{R}_{f} 0.52(\mathrm{Hex} / \mathrm{EtOAc}, 4 / 1)$.

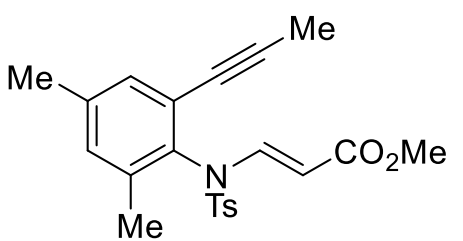

methyl(E)-3-((N-(2,4-dimethyl-6-(prop-1-yn-1-yl)phenyl)-4-methylpheny 1)sulfonamido)acrylate, $\mathbf{3 b}$; viscous oil (from $5 \mathrm{mmol}$ of 4,6-dimethyl-2iodoaniline to 3b, $974 \mathrm{mg}, 49 \%) ;{ }^{1} \mathbf{H}$ NMR (600 $\left.\mathbf{M H z}, \mathbf{C D C l}_{3}\right) \delta 8.23(\mathrm{~d}$, $J=13.6 \mathrm{~Hz}, 1 \mathrm{H}), 7.73(\mathrm{~d}, J=8.2 \mathrm{~Hz}, 2 \mathrm{H}), 7.31(\mathrm{~d}, J=8.2 \mathrm{~Hz}, 2 \mathrm{H}), 7.05$ (s, 2H), $4.53(\mathrm{~d}, J=13.6 \mathrm{~Hz}, 1 \mathrm{H}), 3.67$ (s, 3H), 2.43 (s, 3H), 2.29 (s, 3H), 2.14 (s, 3H), $1.51(\mathrm{~s}, 3 \mathrm{H}) ;{ }^{13} \mathbf{C}$ NMR (151 MHz, $\left.\mathbf{C D C l}_{3}\right) \delta 168.1,144.6,142.8,139.8,139.4,136.8,132.5,132.4,132.2,129.9,128.6$, 124.5, 98.8, 90.2, 76.3, 51.4, 21.8, 21.1, 18.6, 4.5; IR (neat): $v_{\max }=2972,2231,1711,1623,1369,1169$, 898, 734, $576 \mathrm{~cm}^{-1}$; HRMS (EI) m/z: [M+] Calcd for $\mathrm{C}_{22} \mathrm{H}_{23} \mathrm{NO}_{4} \mathrm{~S}$ 397.1348, found 397.1349; $\boldsymbol{R}_{\boldsymbol{f}} 0.52$ (Hex/EtOAc, 4/1). 


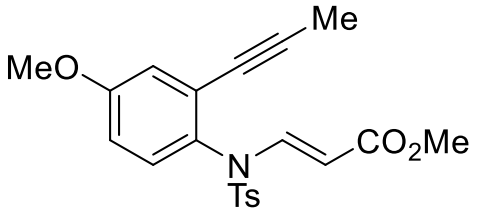

methyl(E)-3-((N-(4-methoxy-2-(prop-1-yn-1-yl)phenyl)-4-methylpheny 1)sulfonamido)acrylate, 3c; viscous oil (from $5 \mathrm{mmol}$ of 4-methoxy-2iodoaniline to $3 \mathbf{c}, 1.1 \mathrm{~g}, 57 \%) ;{ }^{1} \mathbf{H}$ NMR $\left(600 \mathbf{~ M H z}, \mathbf{C D C l}_{3}\right) \delta 8.27(\mathrm{~d}, J$ $=13.7 \mathrm{~Hz}, 1 \mathrm{H}), 7.58(\mathrm{~d}, J=8.1 \mathrm{~Hz}, 2 \mathrm{H}), 7.26(\mathrm{~d}, J=8.1 \mathrm{~Hz}, 2 \mathrm{H}), 6.90$ $(\mathrm{d}, J=8.8 \mathrm{~Hz}, 1 \mathrm{H}), 6.88(\mathrm{~d}, J=2.9 \mathrm{~Hz}, 1 \mathrm{H}), 6.80(\mathrm{dd}, J=8.8,2.9 \mathrm{~Hz}, 1 \mathrm{H}), 4.54(\mathrm{~d}, J=13.7 \mathrm{~Hz}, 1 \mathrm{H}), 3.77$ (s, 3H), 3.64 (s, 3H), 2.40 (s, 3H), 1.70 (s, 3H) ${ }^{13}$ C NMR (151 MHz, CDCl $) \delta$ 167.9, 160.2, 144.7, 143.4, 135.6, 131.7, 129.8, 128.4, 128.2, 126.1, 118.3, 115.1, 98.0, 91.6, 75.3, 55.7, 51.3, 21.7, 4.5; IR (neat): $v_{\max }=2970,2361,1718,1625,1374,1167,577 \mathrm{~cm}^{-1}$; HRMS (EI) m/z: [M+] Calcd for $\mathrm{C}_{21} \mathrm{H}_{21} \mathrm{NO}_{5} \mathrm{~S}$ 399.1140, found 399.1143; $\boldsymbol{R}_{f} 0.51$ (Hex/EtOAc, 4/1).

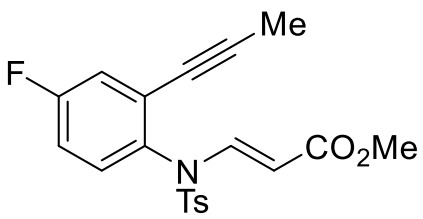

methyl(E)-3-((N-(4-fluoro-2-(prop-1-yn-1-yl)phenyl)-4-methylphenyl)sulfo namido)acrylate, $\mathbf{3 d}$; white solid (from $5 \mathrm{mmol}$ of 4-fluoro-2-iodoaniline to 3d, $970 \mathrm{mg}, 50 \%)$; melting point $\left.98-100{ }^{\circ} \mathrm{C} ;{ }^{1} \mathbf{H} \mathbf{~ N M R ~ ( 6 0 0 ~} \mathbf{M H z}, \mathbf{C D C l}_{3}\right) \delta$ $8.26(\mathrm{~d}, J=13.7 \mathrm{~Hz}, 1 \mathrm{H}), 7.61(\mathrm{~d}, J=7.2 \mathrm{~Hz}, 2 \mathrm{H}), 7.29(\mathrm{~d}, J=7.2 \mathrm{~Hz}, 2 \mathrm{H})$, $7.10(\mathrm{~d}, J=8.5 \mathrm{~Hz}, 1 \mathrm{H}), 7.01-7.0(\mathrm{~m}, 2 \mathrm{H}), 4.54(\mathrm{~d}, J=13.7 \mathrm{~Hz}, 1 \mathrm{H}), 3.68(\mathrm{~s}, 3 \mathrm{H}), 2.44(\mathrm{~s}, 3 \mathrm{H}), 1.74(\mathrm{~s}$, 3H); ${ }^{13}$ C NMR (151 MHz, CDCl $) \delta 167.8,162.7\left(\mathrm{~d},{ }^{1} J_{C-F}=251.3 \mathrm{~Hz}\right), 145.0,143.0,135.5,132.6\left(\mathrm{~d},{ }^{2} J_{C-}\right.$ $\left.{ }_{F}=9.6 \mathrm{~Hz}\right), 132.0\left(\mathrm{~d},{ }^{2} J_{C-F}=3.2 \mathrm{~Hz}\right), 130.0,128.3,127.3\left(\mathrm{~d}, J_{C-F}=10.7 \mathrm{~Hz}\right), 120.7\left(\mathrm{~d}, J_{C-F}=24.0 \mathrm{~Hz}\right)$, $116.4\left(\mathrm{~d}, J_{C-F}=22.8 \mathrm{~Hz}\right), 99.4,93.9,74.0,51.5,21.8,4.6$; IR (neat): $v_{\max }=2970,2361,1718,1623,1367$, 1169, 897, $567 \mathrm{~cm}^{-1} ;$ HRMS (EI) m/z: [M+] Calcd for $\mathrm{C}_{20} \mathrm{H}_{18} \mathrm{FNO}_{4} \mathrm{~S}$ 387.0941, found 387.0942; $\boldsymbol{R}_{f} 0.52$ (Hex/EtOAc, 4/1).

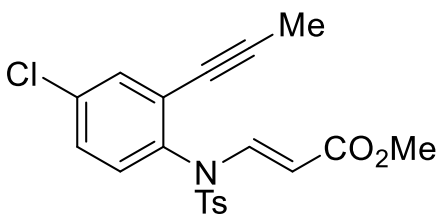

methyl(E)-3-((N-(4-chloro-2-(prop-1-yn-1-yl)phenyl)-4-methylphenyl)sul fonamido)acrylate, $\mathbf{3 e}$; viscous oil (from $5 \mathrm{mmol}$ of 4-chloro-2-iodoaniline to $3 \mathbf{e}, 1.3 \mathrm{~g}, 66 \%) ;{ }^{1} \mathbf{H}$ NMR (600 MHz, $\left.\mathbf{C D C l}_{3}\right) \delta 8.24(\mathrm{~d}, J=13.7 \mathrm{~Hz}, 1 \mathrm{H})$, $7.60(\mathrm{~d}, J=8.5 \mathrm{~Hz}, 2 \mathrm{H}), 7.38(\mathrm{~d}, J=2.5 \mathrm{~Hz}, 1 \mathrm{H}), 7.29(\mathrm{~d}, J=8.5 \mathrm{~Hz}, 2 \mathrm{H})$, $7.27(\mathrm{dd}, J=8.4,2.5 \mathrm{~Hz}, 1 \mathrm{H}), 6.97(\mathrm{~d}, J=8.4 \mathrm{~Hz}, 1 \mathrm{H}), 4.54(\mathrm{~d}, J=13.7 \mathrm{~Hz}, 1 \mathrm{H}), 3.67(\mathrm{~s}, 3 \mathrm{H}), 2.43(\mathrm{~s}, 3 \mathrm{H})$, 1.73 (s, 3H); ${ }^{13} \mathbf{C}$ NMR (151 MHz, CDCl $) \delta$ 167.7, 145.6, 142.7, 135.9, 135.4, 134.4, 133.8, 131.0, 130.0, 129.2, 128.2, 126.8, 101.1, 93.6, 74.3, 50.1, 22.3, 4.6; IR (neat): $v_{\max }=2970,2361,1716,1624,1367,1168$, 682, $565 \mathrm{~cm}^{-1}$; HRMS (EI) m/z: [M+] Calcd for $\mathrm{C}_{20} \mathrm{H}_{18} \mathrm{ClNO}_{4} \mathrm{~S} 403.0645$, found 403.0647; $\boldsymbol{R}_{\boldsymbol{f}} 0.5$ (Hex/EtOAc, 4/1). 


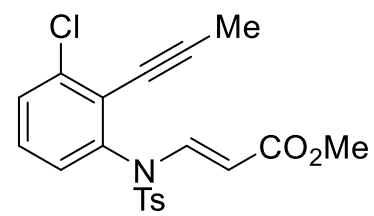

methyl(E)-3-((N-(3-chloro-2-(prop-1-yn-1-yl)phenyl)-4-methylphenyl)sulfona mido)acrylate, 3f; viscous oil (from $5 \mathrm{mmol}$ of 3-chloro-2-iodoaniline to 3f, 947 $\mathrm{mg}, 47 \%) ;{ }^{1} \mathbf{H}$ NMR (600 MHz, $\left.\mathbf{C D C l}_{3}\right) \delta 8.23(\mathrm{~d}, J=13.8 \mathrm{~Hz}, 1 \mathrm{H}), 7.63(\mathrm{~d}, J=$ $8.4 \mathrm{~Hz}, 2 \mathrm{H}), 7.47(\mathrm{~d}, J=8.0 \mathrm{~Hz}, 1 \mathrm{H}), 7.31(\mathrm{~d}, J=8.4 \mathrm{~Hz}, 2 \mathrm{H}), 7.23(\mathrm{t}, J=7.0$ $\mathrm{Hz}, 1 \mathrm{H}), 6.96(\mathrm{~d}, J=8.0 \mathrm{~Hz}, 1 \mathrm{H}), 4.55(\mathrm{~d}, J=13.8 \mathrm{~Hz}, 1 \mathrm{H}), 3.68(\mathrm{~s}, 3 \mathrm{H}), 2.44(\mathrm{~s}, 3 \mathrm{H}), 1.82(\mathrm{~s}, 3 \mathrm{H}) ;{ }^{13} \mathrm{C}$ NMR (151 MHz, $\left.\mathbf{C D C l}_{3}\right) \delta$ 167.7, 145.1, 142.7, 137.7, 137.6, 135.8, 135.6, 130.8, 130.1, 129.0, 128.2, 125.3, 99.7, 98.2, 72.7, 51.5, 21.8, 4.9; IR (neat): $v_{\max }=2972,2231,1711,1623,1369,1169,1130,898$, 734, 703, 671, $576 \mathrm{~cm}^{-1}$; HRMS (EI) m/z: [M+] Calcd for $\mathrm{C}_{20} \mathrm{H}_{18} \mathrm{ClNO}_{4} \mathrm{~S}$ 403.0645, found 403.0645; $\boldsymbol{R}_{\boldsymbol{f}}$ $0.5(\mathrm{Hex} / \mathrm{EtOAc}, 4 / 1)$.

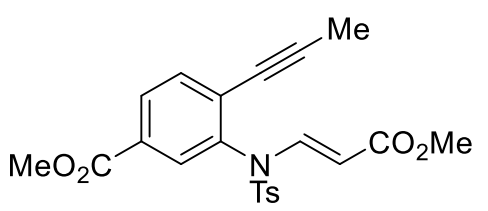

methyl(E)-3-((N-(3-methoxy-3-oxoprop-1-en-1-yl)-4-methylphenyl)sulf onamido)-4-(prop-1-yn-1-yl)benzoate, 3g; white solid (from $5 \mathrm{mmol}$ of methyl 3-amino-4-iodobenzoate to $3 \mathrm{~g}, 1.3 \mathrm{~g}$, 59\%); melting point 134$136{ }^{\circ} \mathrm{C} ;{ }^{1} \mathbf{H}$ NMR $\left(\mathbf{6 0 0} \mathbf{M H z}, \mathbf{C D C l}_{3}\right) \delta 8.26(\mathrm{~d}, J=13.8 \mathrm{~Hz}, 1 \mathrm{H}), 8.01$ $(\mathrm{dd}, J=8.1,1.7 \mathrm{~Hz}, 1 \mathrm{H}), 7.69(\mathrm{~d}, J=1.7 \mathrm{~Hz}, 1 \mathrm{H}), 7.62(\mathrm{~d}, J=8.1 \mathrm{~Hz}, 2 \mathrm{H}), 7.49$ (d, $J=8.1 \mathrm{~Hz}, 1 \mathrm{H}), 7.30$ $(\mathrm{d}, J=8.1 \mathrm{~Hz}, 2 \mathrm{H}), 4.51(\mathrm{~d}, J=13.8 \mathrm{~Hz}, 1 \mathrm{H}), 3.89(\mathrm{~s}, 3 \mathrm{H}), 3.67(\mathrm{~s}, 3 \mathrm{H}), 2.44(\mathrm{~s}, 3 \mathrm{H}), 1.79(\mathrm{~s}, 3 \mathrm{H}) ;{ }^{13} \mathbf{C}$ NMR (151 MHz, CDCl $) \delta$ 168.0, 165.5, 145.1, 142.2, 136.3, 135.6, 134.1, 131.6, 130.8, 130.7, 130.1, 129.0, 128.3, 99.6, 95.7 75.2, 54.6, 51.5, 21.4, 5.6; IR (neat): $v_{\max }=2952,2354,1720,1624,14363,1370$, 1170, 1140, 1087, 766, 684, $582 \mathrm{~cm}^{-1}$; HRMS (EI) m/z: [M+] Calcd for $\mathrm{C}_{22} \mathrm{H}_{21} \mathrm{NO}_{6} \mathrm{~S}$ 427.1090, found 427.1092; $\boldsymbol{R}_{f} 0.41$ (Hex/EtOAc, 4/1).

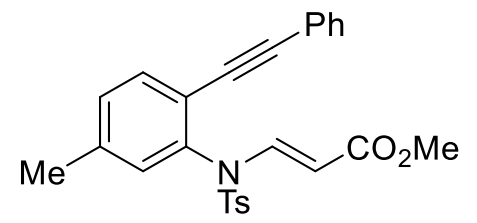

methyl(E)-3-((4-methyl- $N$-(5-methyl-2-(phenylethynyl)phenyl)phenyl)su lfonamido)acrylate, $\mathbf{3 h}$; white solid (from $5 \mathrm{mmol}$ of 5-methyl-2iodoaniline to $3 \mathbf{h}, 1.2 \mathrm{~g}, 54 \%)$; melting point $140-142{ }^{\circ} \mathrm{C} ;{ }^{1} \mathbf{H}$ NMR (600 $\left.\mathbf{M H z}, \mathbf{C D C l}_{3}\right) \delta 8.33(\mathrm{~d}, J=13.7 \mathrm{~Hz}, 1 \mathrm{H}), 7.59(\mathrm{~d}, J=8.4 \mathrm{~Hz}, 2 \mathrm{H}), 7.42$ $(\mathrm{d}, J=7.8 \mathrm{~Hz}, 1 \mathrm{H}), 7.31-7.25(\mathrm{~m}, 3 \mathrm{H}), 7.23(\mathrm{dd}, J=7.9,1.8 \mathrm{~Hz}, 1 \mathrm{H}), 7.21-7.17(\mathrm{~m}, 2 \mathrm{H}), 7.08-7.04(\mathrm{~m}$, 3H), $4.67(\mathrm{~d}, J=13.7 \mathrm{~Hz}, 1 \mathrm{H}), 3.67(\mathrm{~s}, 3 \mathrm{H}), 2.38(\mathrm{~s}, 3 \mathrm{H}), 2.20(\mathrm{~s}, 3 \mathrm{H}) ;{ }^{13} \mathbf{C}$ NMR (151 MHz, CDCl $\left.\mathbf{3}\right) \delta$ $167.9,144.9,142.0,140.5,135.0,135.5,133.5,131.9,131.7,130.9,130.0,128.6,128.3,128.1,122.0$, 121.1, 99.6, 94.3, 85.2, 51.5, 21.7, 21.5; IR (neat): $v_{\max }=2949,2361,1710,1624,1365,1164,569 \mathrm{~cm}^{-1}$; HRMS (EI) m/z: [M+] Calcd for $\mathrm{C}_{26} \mathrm{H}_{23} \mathrm{NO}_{4} \mathrm{~S} 445.1348$, found 445.1348; $\boldsymbol{R}_{f} 0.51$ (Hex/EtOAc, 4/1). 


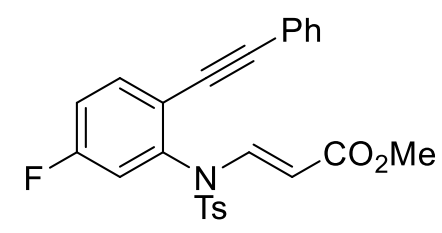

methyl(E)-3-((N-(5-fluoro-2-(phenylethynyl)phenyl)-4-methylphenyl)sulfo namid) acrylate, $\mathbf{3 i}$; white solid (from $5 \mathrm{mmol}$ of 5-fluoro-2-iodoaniline to $\mathbf{3 i}$, $1.0 \mathrm{~g}, 45 \%)$; melting point $122-124{ }^{\circ} \mathrm{C} ;{ }^{1} \mathbf{H}$ NMR (600 $\left.\mathbf{~ M H z}, \mathbf{C D C l}_{3}\right) \delta 8.30$ $(\mathrm{d}, J=13.8 \mathrm{~Hz}, 1 \mathrm{H}), 7.60(\mathrm{~d}, J=8.2 \mathrm{~Hz}, 2 \mathrm{H}), 7.53(\mathrm{dd}, J=8.6,6.0 \mathrm{~Hz}, 1 \mathrm{H})$, 7.37-7.32 (m, 1H), $7.30(\mathrm{~d}, J=7.0 \mathrm{~Hz}, 2 \mathrm{H}), 7.23(\mathrm{~d}, J=7.0 \mathrm{~Hz}, 2 \mathrm{H}), 7.17(\mathrm{~d}, J=10.0 \mathrm{~Hz}, 1 \mathrm{H}), 7.10(\mathrm{~d}, J$ $=8.2 \mathrm{~Hz}, 2 \mathrm{H}), 6.97(\mathrm{dd}, J=8.6,2.5 \mathrm{~Hz}, 1 \mathrm{H}), 4.69(\mathrm{~d}, J=13.8 \mathrm{~Hz}, 1 \mathrm{H}), 3.68(\mathrm{~s}, 3 \mathrm{H}), 2.24(\mathrm{~s}, 3 \mathrm{H}) ;{ }^{13} \mathrm{C}$ NMR (151 MHz, CDCl $) \delta 167.6,163.8(\mathrm{~d}, J=176.6 \mathrm{~Hz}), 161.5,145.3,142.4,136.0,135.2,134.0$ (d, $J$ $=8.9 \mathrm{~Hz}), 133.9(\mathrm{~d}, J=8.9 \mathrm{~Hz}), 131.7,130.2,128.4,128.2,128.1,122.6,118.9(\mathrm{~d}, J=23.5 \mathrm{~Hz}), 117.7(\mathrm{~d}$, $J=21.7 \mathrm{~Hz}$ ), 100.0, 84.1, 51.6, 21.7; IR (neat): $v_{\max }=2970,2361,1718,1625,1362,1167,938,576 \mathrm{~cm}^{-}$ ${ }^{1}$; HRMS (EI) m/z: $\left[\mathrm{M}^{+}\right]$Calcd for $\mathrm{C}_{25} \mathrm{H}_{20} \mathrm{FNO}_{4} \mathrm{~S}$ 449.1097, found 449.1093; $\boldsymbol{R}_{f} 0.52$ (Hex/EtOAc, 4/1).

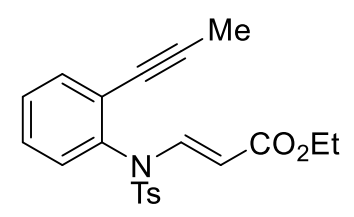

ethyl(E)-3-((4-methyl- $N$-(2-(prop-1-yn-1-yl)phenyl)phenyl)sulfonamido)acryla, $3 \mathbf{j}$; white solid (from $5 \mathrm{mmol}$ of 2-iodoaniline to $\mathbf{3 j}$, $1.2 \mathrm{~g}$, 61\%); melting point 96$98{ }^{\circ} \mathrm{C} ;{ }^{1} \mathbf{H}$ NMR $\left(600 \mathbf{~ M H z}, \mathbf{C D C l}_{3}\right) \delta 8.26(\mathrm{~d}, J=13.6 \mathrm{~Hz}, 1 \mathrm{H}), 7.58(\mathrm{~d}, J=8.3$ $\mathrm{Hz}, 2 \mathrm{H}), 7.38(\mathrm{~d}, J=7.7 \mathrm{~Hz}, 1 \mathrm{H}), 7.33-7.27(\mathrm{~m}, 2 \mathrm{H}), 7.25(\mathrm{~d}, J=8.3 \mathrm{~Hz}, 2 \mathrm{H}), 7.01$ $(\mathrm{d}, J=7.7 \mathrm{~Hz}, 1 \mathrm{H}), 4.51(\mathrm{~d}, J=13.6 \mathrm{~Hz}, 1 \mathrm{H}), 4.10(\mathrm{q}, J=7.2, \mathrm{~Hz}, 2 \mathrm{H}), 2.39(\mathrm{~s}, 3 \mathrm{H}), 1.71(\mathrm{~s}, 3 \mathrm{H}), 1.22(\mathrm{t}$, $J=7.2, \mathrm{~Hz}, 3 \mathrm{H}) ;{ }^{13} \mathbf{C}$ NMR (151 MHz, $\left.\mathbf{C D C l}_{3}\right) \delta 167.9,145.7,142.8,135.9,135.6,134.3,130.6,129.8$, 129.8, 128.9, 128.1, 125.3, 98.5, 91.2, 71.2, 60.1, 21.7, 14.4, 3.6; IR (neat): $v_{\max }=2981,2361,1706,1623$, 1487, 1367, 1170, 1131, 1088, $578 \mathrm{~cm}^{-1}$; HRMS (EI) m/z: [M+] Calcd for $\mathrm{C}_{21} \mathrm{H}_{21} \mathrm{NO}_{4} \mathrm{~S} 383.1191$, found $383.1187 ; \boldsymbol{R}_{f} 0.41(\mathrm{Hex} / \mathrm{EtOAc}, 4 / 1)$.

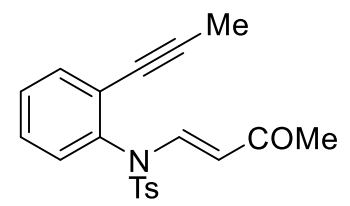

(E)-4-methyl- $N$-(3-oxobut-1-en-1-yl)- $N$-(2-(prop-1-yn-1-yl)phenyl)benzenesulf onamide, 3k; colorless oil (from 5 mmol of 2-iodoaniline to $3 \mathbf{k}, 830 \mathrm{mg}, 47 \%$ ); ${ }^{1} \mathbf{H}$ NMR (600 MHz, CDCl $) \delta .20(\mathrm{~d}, J=13.9 \mathrm{~Hz}, 1 \mathrm{H}), 7.59(\mathrm{~d}, J=8.3 \mathrm{~Hz}, 2 \mathrm{H}), 7.40(\mathrm{dd}$, $J=7.6,1.8 \mathrm{~Hz}, 1 \mathrm{H}), 7.35(\mathrm{td}, J=7.6,7.5,1.5 \mathrm{~Hz}, 1 \mathrm{H}), 7.32(\mathrm{td}, J=7.7,7.5,1.8 \mathrm{~Hz}, 1 \mathrm{H})$, $7.28(\mathrm{~d}, J=8.3 \mathrm{~Hz}, 2 \mathrm{H}), 7.05(\mathrm{dd}, J=7.7,1.5 \mathrm{~Hz}, 1 \mathrm{H}), 4.90(\mathrm{~d}, J=13.9 \mathrm{~Hz}, 1 \mathrm{H}), 2.43(\mathrm{~s}, 3 \mathrm{H}), 2.16(\mathrm{~s}, 3 \mathrm{H}), 1.72(\mathrm{~s}$, 3H); ${ }^{13}$ C NMR (151 MHz, $\mathbf{C D C l}_{3}$ ) $\delta 198.3$, 145.6, 141.0, 137.6, 136.1, 133.7, 130.3, 128.1, 127.4, 126.7, 125.1, 124.5, 120.6, 116.1, 114.5, 27.7, 22.5, 12.0; IR (neat): $v_{\max }=2918,2361,1684,1657,1581,1486,1365,1167$, 1087,861, 682, $565 \mathrm{~cm}^{-1}$; HRMS (EI) m/z: [M+] Calcd for $\mathrm{C}_{20} \mathrm{H}_{19} \mathrm{NO}_{3} \mathrm{~S}$ 353.1086, found 353.1083; $\boldsymbol{R}_{\boldsymbol{f}}$ $0.51(\mathrm{Hex} / \mathrm{EtOAc}, 4 / 1)$. 


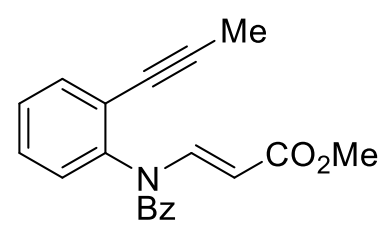

$=14.1 \mathrm{~Hz}, 1 \mathrm{H}), 3.67$ (s, 3H), 2.00 (s, 3H). ${ }^{13} \mathbf{C}$ NMR (151 MHz, CDCl $\left.\mathbf{3}\right) \delta$ 169.3, 167.1, 144.0, 140.2, 134.4, 133.6, 131.1, 129.2, 128.9, 128.7, 128.1, 124.3, 102.2, 92.5, 77.5, 75.5, 51.4, 4.7.; IR (neat): $v_{\max }=2970$, 2360, 1717, 1682, 1623, 1365, 1147, $568 \mathrm{~cm}^{-1}$; HRMS (EI) m/z: [M $\left.{ }^{+}\right]$Calcd for $\mathrm{C}_{20} \mathrm{H}_{17} \mathrm{NO}_{3} 319.1208$, found $319.1210 ; \boldsymbol{R}_{f} 0.51$ (Hex/EtOAc, 4/1).

\section{Analytical data for indoles (2 and 4):}

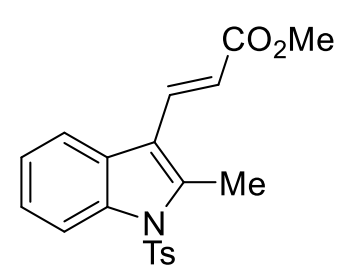

methyl (E)-3-(2-methyl-1-tosyl-1H-indol-3-yl)acrylate, 2a: white solid (106 mg, 96\%); melting point $144-146{ }^{\circ} \mathrm{C} ;{ }^{1} \mathbf{H}$ NMR (600 $\left.\mathbf{~ M H z ,} \mathbf{C D C l}_{3}\right) \delta 8.27(\mathrm{~d}, J=8.3$ $\mathrm{Hz}, 1 \mathrm{H}), 7.83(\mathrm{~d}, J=16.1 \mathrm{~Hz}, 1 \mathrm{H}), 7.76(\mathrm{~d}, J=7.5 \mathrm{~Hz}, 1 \mathrm{H}), 7.68(\mathrm{~d}, J=8.4 \mathrm{~Hz}$, $2 \mathrm{H}), 7.34(\mathrm{td}, J=7.7,1.08 \mathrm{~Hz}, 1 \mathrm{H}), 7.31(\mathrm{t}, J=7.5 \mathrm{~Hz}, 1 \mathrm{H}), 7.22(\mathrm{~d}, J=8.4 \mathrm{~Hz}$, $2 \mathrm{H}), 6.50(\mathrm{~d}, J=16.1 \mathrm{~Hz}, 1 \mathrm{H}), 3.81(\mathrm{~s}, 3 \mathrm{H}), 2.72(\mathrm{~s}, 3 \mathrm{H}), 2.35(\mathrm{~s}, 3 \mathrm{H}) ;{ }^{13} \mathbf{C} \mathbf{N M R}$

$\left(\mathbf{1 5 1} \mathbf{M H z}, \mathbf{C D C l}_{3}\right) \delta 167.0,145.5,140.0,136.9,136.1,135.0,130.3,127.6,126.7,125.0,124.4,119.0$, 118.2, 116.1, 114.9, 51.9, 21.8, 13.4; IR (neat): $v_{\max }=2947,1738,1631,1434,1371,1172,1025,569 \mathrm{~cm}^{-}$ ${ }^{1}$; HRMS (EI) m/z: [M+] Calcd for $\mathrm{C}_{20} \mathrm{H}_{19} \mathrm{NO}_{4} \mathrm{~S} 369.1035$, found 369.1031; $\boldsymbol{R}_{f} 0.56$ (Hex/EtOAc, 4/1).

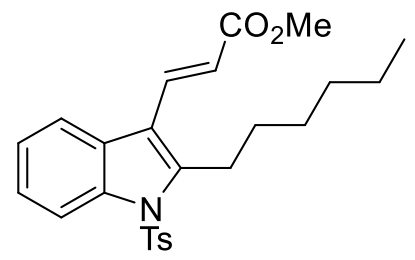

methyl (E)-3-(2-hexyl-1-tosyl-1H-indol-3-yl)acrylate, 2b: white solid (112 mg, 85\%); melting point $88-89{ }^{\circ} \mathrm{C} ;{ }^{1} \mathbf{H}$ NMR (600 $\left.\mathbf{M H z}, \mathbf{C D C l}_{3}\right) \delta 8.25(\mathrm{~d}, J=7.4$ $\mathrm{Hz}, 1 \mathrm{H}), 7.83(\mathrm{~d}, J=16.1 \mathrm{~Hz}, 1 \mathrm{H}), 7.77(\mathrm{~d}, J=7.2 \mathrm{~Hz}, 1 \mathrm{H}), 7.63(\mathrm{~d}, \mathrm{~J}=8.4$ $\mathrm{Hz}, 2 \mathrm{H}), 7.33(\mathrm{td}, J=7.3,1.4 \mathrm{~Hz}, 1 \mathrm{H}), 7.31(\mathrm{td}, J=7.3,1.2 \mathrm{~Hz}, 1 \mathrm{H}), 7.19(\mathrm{~d}$, $J=8.4 \mathrm{~Hz}, 2 \mathrm{H}), 6.52(\mathrm{~d}, J=16.1 \mathrm{~Hz}, 1 \mathrm{H}), 3.82(\mathrm{~s}, 3 \mathrm{H}), 3.16(\mathrm{t}, J=7.8 \mathrm{~Hz}, 2 \mathrm{H}), 2.33(\mathrm{~s}, 3 \mathrm{H}), 1.76-1.63(\mathrm{~m}$, 2H), $1.42(\mathrm{tt}, J=7.3 \mathrm{~Hz}, 2 \mathrm{H}), 1.32(\mathrm{~m}, 4 \mathrm{H}), 0.92-0.88(\mathrm{~m}, 3 \mathrm{H}) ;{ }^{13} \mathbf{C} \mathbf{N M R}\left(\mathbf{1 5 1} \mathbf{M H z}, \mathbf{C D C l}_{\mathbf{3}}\right) \delta 168.0$, 145.4, 140.7, 137.0, 136.1 135.0, 130.1, 127.7, 126.5, 125.0, 124.5, 120.1, 118.1, 116.2, 115.4, 51.9, 31.6, 31.5, 29.4, 26.8, 22.8, 21.8, 14.3; IR (neat): $v_{\max }=2928,1718,1630,1451,1371,1169,1089,572 \mathrm{~cm}^{-1}$; HRMS (EI) m/z: $\left[\mathrm{M}^{+}\right]$Calcd for $\mathrm{C}_{25} \mathrm{H}_{29} \mathrm{NO}_{4} \mathrm{~S} 439.1817$, found 439.1821; $\boldsymbol{R}_{f} 0.54$ (Hex/EtOAc, 4/1). 


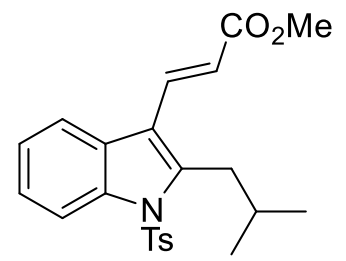

methyl (E)-3-(2-isobutyl-1-tosyl-1H-indol-3-yl)acrylate, 2c: white solid (77 mg, $62 \%)$; melting point $84-86{ }^{\circ} \mathrm{C} ;{ }^{1} \mathbf{H}$ NMR $\left(600 \mathbf{~ M H z}, \mathbf{C D C l}_{3}\right) \delta 8.23(\mathrm{~d}, J=7.6 \mathrm{~Hz}$, $1 \mathrm{H}), 7.83(\mathrm{~d}, J=16.1 \mathrm{~Hz}, 1 \mathrm{H}), 7.78(\mathrm{~d}, J=7.2 \mathrm{~Hz}, 1 \mathrm{H}), 7.58(\mathrm{~d}, J=8.4 \mathrm{~Hz}, 2 \mathrm{H})$, $7.33(\mathrm{td}, J=7.6,1.4 \mathrm{~Hz}, 1 \mathrm{H}), 7.30(\mathrm{dd}, J=7.2,1.4 \mathrm{~Hz}, 1 \mathrm{H}), 7.17(\mathrm{~d}, J=8.4 \mathrm{~Hz}$, 2H), $6.54(\mathrm{~d}, J=16.1 \mathrm{~Hz}, 1 \mathrm{H}), 3.81(\mathrm{~s}, 3 \mathrm{H}), 3.05$ (d, $J=7.1 \mathrm{~Hz}, 2 \mathrm{H}), 2.32$ (s, 3H), 2.23 (dp, $J=13.2,6.6$ $\mathrm{Hz}, 1 \mathrm{H}), 0.97$ (d, $J=6.7 \mathrm{~Hz}, 6 \mathrm{H}) ;{ }^{13} \mathbf{C}$ NMR (151 MHz, $\left.\mathbf{C D C l}_{3}\right)$ 168.0, 145.3, 144.6, 137.2, 136.6, 135.9, $130.1,127.8,126.5,125.1,124.6,120.2,118.2,117.6,115.7,51.9,35.4,30.9,22.6,21.8$; IR (neat): $v_{\max }=$ 2931, 1737, 1628, 1451, 1366, 1170, 1087, $573 \mathrm{~cm}^{-1}$; HRMS (EI) m/z: [M+] Calcd for $\mathrm{C}_{23} \mathrm{H}_{25} \mathrm{NO}_{4} \mathrm{~S}$ 411.1504, found 411.1504; $\boldsymbol{R}_{f} 0.61(\mathrm{Hex} / \mathrm{EtOAc}, 4 / 1)$.

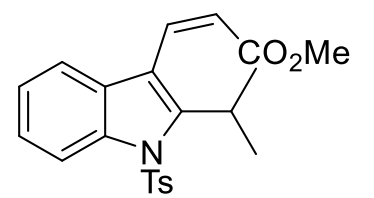

methyl (Z)-3-(2-isopropyl-1-tosyl-1H-indol-3-yl)acrylate, 2d: white solid (63 mg, $53 \%)$; melting point $81-83{ }^{\circ} \mathrm{C}$; ${ }^{1} \mathbf{H}$ NMR $\left(600 ~ \mathbf{M H z}, \mathbf{C D C l}_{3}\right) \delta 8.22(\mathrm{~d}, J=8.4 \mathrm{~Hz}$, $1 \mathrm{H}), 7.56(\mathrm{~d}, J=8.4 \mathrm{~Hz}, 2 \mathrm{H}), 7.25(\mathrm{td}, J=8.4,1.6 \mathrm{~Hz}, 1 \mathrm{H}), 7.22-7.17(\mathrm{~m}, 3 \mathrm{H})$, 7.17-7.15 (m, 1H), $7.10(\mathrm{~d}, J=11.8 \mathrm{~Hz}, 1 \mathrm{H}), 6.18(\mathrm{~d}, J=11.8 \mathrm{~Hz}, 1 \mathrm{H}), 3.95(\mathrm{~m}, 1 \mathrm{H}), 3.38(\mathrm{~s}, 3 \mathrm{H}), 2.35(\mathrm{~s}$, 3H), $1.24(\mathrm{~d}, J=7.0 \mathrm{~Hz}, 6 \mathrm{H}) ;{ }^{13} \mathbf{C}$ NMR (151 MHz, $\left.\mathbf{C D C l}_{3}\right) \delta 166.0,144.8,143.2,137.0,136.6,136.0$, 130.0, 129.4, 126.3, 124.5, 123.7, 123.7, 118.9, 117.4, 115.7, 51.4, 27.1, 22.0, 21.8; IR (neat): $v_{\max }=2954$, 1737, 1634, 1449, 1365, 1173, 1091, $578 \mathrm{~cm}^{-1}$; HRMS (EI) m/z: [M+] Calcd for $\mathrm{C}_{22} \mathrm{H}_{23} \mathrm{NO}_{4} \mathrm{~S} 397.1348$, found 397.1346; $\boldsymbol{R}_{f} 0.60$ (Hex/EtOAc, 4/1).

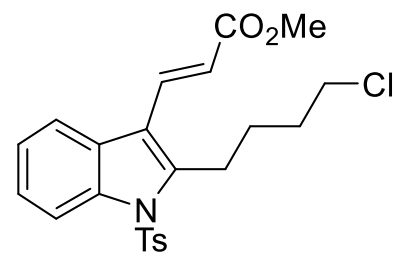

methyl (E)-3-(2-(4-chlorobutyl)-1-tosyl-1H-indol-3-yl)acrylate, 2e: white solid (120 mg, 90\%); melting point 90-92 ${ }^{\circ} \mathrm{C} ;{ }^{1} \mathbf{H}$ NMR (600 $\left.\mathbf{M H z}, \mathbf{C D C l}_{3}\right) \delta 8.24$ $(\mathrm{d}, J=7.6 \mathrm{~Hz}, 1 \mathrm{H}), 7.82(\mathrm{~d}, J=16.1 \mathrm{~Hz}, 1 \mathrm{H}), 7.77(\mathrm{~d}, J=7.5 \mathrm{~Hz}, 1 \mathrm{H}), 7.62(\mathrm{~d}$, $J=8.4 \mathrm{~Hz}, 2 \mathrm{H}), 7.34(\mathrm{td}, J=7.6,1.2 \mathrm{~Hz}, 1 \mathrm{H}), 7.31(\mathrm{td}, J=7.5,1.2 \mathrm{~Hz}, 1 \mathrm{H})$, $7.19(\mathrm{~d}, J=8.4 \mathrm{~Hz}, 2 \mathrm{H}), 6.53(\mathrm{~d}, J=16.1 \mathrm{~Hz}, 1 \mathrm{H}), 3.82(\mathrm{~s}, 3 \mathrm{H}), 3.56(\mathrm{t}, J=6.1 \mathrm{~Hz}, 2 \mathrm{H}), 3.21(\mathrm{t}, J=7.2$ Hz, 2H), 2.33 (s, 3H), 1.95-1.80 (m, 4H); ${ }^{13} \mathbf{C}$ NMR (151 MHz, $\left.\mathbf{C D C l}_{3}\right) \delta 167.9,145.5,144.1,137.0,135.9$, 135.6, 130.2, 127.6, 126.5, 125.1, 124.6, 120.2, 118.7, 116.7, 115.4, 51.9, 44.6, 32.4, 28.8, 26.0, 21.7; IR (neat): $v_{\max }=2948,1739,1634,1436,1366,1174,1092,768,528 \mathrm{~cm}^{-1} ; \mathbf{H R M S}(\mathrm{EI}) \mathrm{m} / \mathrm{z}:\left[\mathrm{M}^{+}\right]$Calcd for $\mathrm{C}_{23} \mathrm{H}_{24} \mathrm{ClNO}_{4} \mathrm{~S} 445.1115$, found 445.1116; $\boldsymbol{R}_{f} 0.60$ (Hex/EtOAc, 4/1).

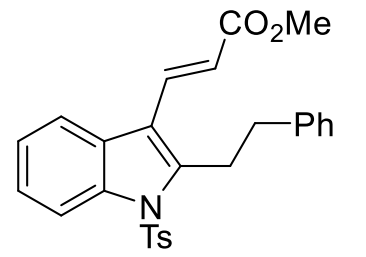

methyl (E)-3-(2-phenethyl-1-tosyl-1H-indol-3-yl)acrylate, 2f: white solid (108 mg, 78\%); melting point $130-132{ }^{\circ} \mathrm{C} ;{ }^{1} \mathbf{H}$ NMR (600 MHz, $\left.\mathbf{C D C l}_{3}\right) \delta 8.29(\mathrm{~d}, J=8.4$ $\mathrm{Hz}, 1 \mathrm{H}), 7.74(\mathrm{~d}, J=7.8 \mathrm{~Hz}, 1 \mathrm{H}), 7.68-7.61(\mathrm{~m}, 3 \mathrm{H}), 7.37$ (t, $J=7.8 \mathrm{~Hz}, 1 \mathrm{H}), 7.32$ (t, $J=7.8 \mathrm{~Hz}, 1 \mathrm{H}), 7.29(\mathrm{t}, J=7.4 \mathrm{~Hz}, 2 \mathrm{H}), 7.25(\mathrm{~d}, J=6.2 \mathrm{~Hz}, 2 \mathrm{H}), 7.20(\mathrm{~m}, 3 \mathrm{H})$, $6.42(\mathrm{~d}, J=16.1 \mathrm{~Hz}, 1 \mathrm{H}), 3.81(\mathrm{~s}, 3 \mathrm{H}), 3.43(\mathrm{t}, J=7.8 \mathrm{~Hz}, 2 \mathrm{H}), 3.04(\mathrm{t}, J=7.8 \mathrm{~Hz}, 2 \mathrm{H}), 2.33(\mathrm{~s}, 3 \mathrm{H}) ;{ }^{13} \mathbf{C}$ 
NMR (151 MHz, CDCl 3 ) $\delta 167.8,145.5,143.2,140.8,137.1,136.0,135.4,130.2,128.9,128.7,127.6$, 126.5, 125.2, 124.6, 120.2, 118.5, 116.0, 115.4, 51.9, 37.5, 29.7, 21.8 (One peak is overlapping); IR (neat): $v_{\max }=2949,1736,1631,1450,1370,1166,1088,570 \mathrm{~cm}^{-1}$; HRMS (EI) m/z: $\left[\mathrm{M}^{+}\right]$Calcd for $\mathrm{C}_{27} \mathrm{H}_{25} \mathrm{NO}_{4} \mathrm{~S} 459.1504$, found $459.1507 ; \boldsymbol{R}_{f} 0.51$ (Hex/EtOAc, 4/1).

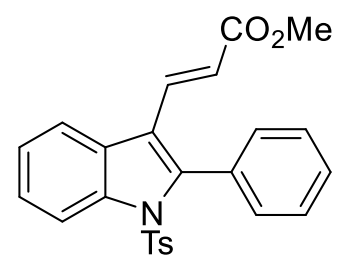

methyl $(E)-3$-(2-phenyl-1-tosyl-1H-indol-3-yl)acrylate, 2g: white solid (119 mg, 92\%); melting point $162-164{ }^{\circ} \mathrm{C} ;{ }^{1} \mathbf{H}$ NMR $\left(600 \mathbf{~ M H z}, \mathbf{C D C l}_{3}\right) \delta 8.42(\mathrm{~d}, J=8.4$ $\mathrm{Hz}, 1 \mathrm{H}), 7.83(\mathrm{~d}, J=7.9 \mathrm{~Hz}, 1 \mathrm{H}), 7.52(\mathrm{t}, J=7.4 \mathrm{~Hz}, 1 \mathrm{H}), 7.46(\mathrm{t}, J=7.8 \mathrm{~Hz}, 3 \mathrm{H})$, 7.41-7.37 (m, 2H), 7.34-7.29 (m, 4H), 7.09 (d, $J=8.4 \mathrm{~Hz}, 2 \mathrm{H}), 6.45(\mathrm{~d}, J=16.3$ $\mathrm{Hz}, 1 \mathrm{H}), 3.72$ (s, 3H), 2.32 (s, 3H); ${ }^{13} \mathbf{C}$ NMR (151 MHz, CDCl 3 ) $\delta$ 167.7, 145.3, 143.2, 137.6, 136.8, 135.4, 131.0, 129.9, 129.8, 129.7, 127.8, 127.1, 125.8, 124.9, 120.6, 118.8, 118.5, 116.4, 51.8, 21.8 (one carbon peak is overlapping); IR (neat): $v_{\max }=2949,1727,1632,1448,1375,1174,1082,572 \mathrm{~cm}^{-1}$; HRMS (EI) m/z: $\left[\mathrm{M}^{+}\right]$Calcd for $\mathrm{C}_{25} \mathrm{H}_{21} \mathrm{NO}_{4} \mathrm{~S} 397.1348$, found 397.1346; $\boldsymbol{R}_{f} 0.69$ (Hex/EtOAc, 8/1).

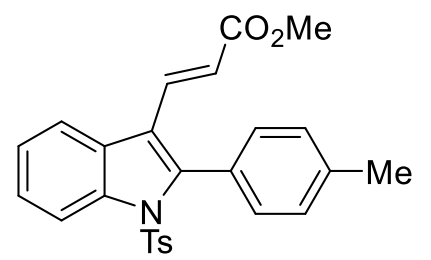

methyl (E)-3-(2-(p-tolyl)-1-tosyl-1H-indol-3-yl)acrylate, $2 \mathbf{h}$ : white solid (110 mg, 82\%); melting point $174-176{ }^{\circ} \mathrm{C} ;{ }^{1} \mathbf{H}$ NMR (600 $\left.\mathbf{M H z}, \mathbf{C D C l}_{3}\right) \delta 8.41$ (d, $J=8.2 \mathrm{~Hz}, 1 \mathrm{H}), 7.82(\mathrm{~d}, J=7.8 \mathrm{~Hz}, 1 \mathrm{H}), 7.44(\mathrm{t}, J=7.6 \mathrm{~Hz}, 1 \mathrm{H}), 7.37(\mathrm{t}, J=$ $7.8 \mathrm{~Hz}, 1 \mathrm{H}), 7.33(\mathrm{~d}, J=8.2 \mathrm{~Hz}, 2 \mathrm{H}), 7.32-7.30(\mathrm{~m}, 1 \mathrm{H}), 7.27(\mathrm{~d}, J=7.6 \mathrm{~Hz}$, 2H), $7.21(\mathrm{~d}, J=8.0 \mathrm{~Hz}, 2 \mathrm{H}), 7.09$ (d, $J=8.0 \mathrm{~Hz}, 2 \mathrm{H}), 6.45$ (d, $J=16.3 \mathrm{~Hz}, 1 \mathrm{H}), 3.72(\mathrm{~s}, 3 \mathrm{H}), 2.47$ (s, 3H), 2.31 (s, 3H); ${ }^{13} \mathbf{C}$ NMR (151 MHz, CDCl $) \delta 167.8,145.2,143.7,139.9,137.6,137.0,135.1,131.8,129.6$, $128.6,127.9,127.1,127.0,125.7,124.9,120.5,118.5,118.4,116.4,51.7,51.2,21.7$; IR (neat): $v_{\max }=2950$, 1733, 1632, 1448, 1374, 1174, 1081, $570 \mathrm{~cm}^{-1}$; HRMS (EI) m/z: $\left[\mathrm{M}^{+}\right]$Calcd for $\mathrm{C}_{26} \mathrm{H}_{23} \mathrm{NO}_{4} \mathrm{~S} 445.1348$, found 445.1350; $\boldsymbol{R}_{f} 0.59$ (Hex/EtOAc, 4/1).

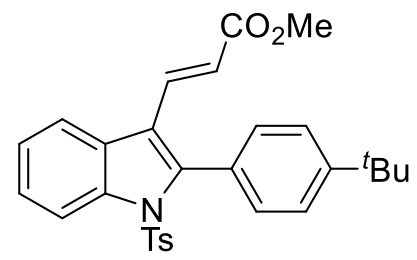

methyl(E)-3-(2-(4-(tert-butyl)phenyl)-1-tosyl-1H-indol-3-yl)acrylate, $2 \mathbf{i}$ : whi te solid (133 mg, 91\%); melting point $132-134{ }^{\circ} \mathrm{C}$; ${ }^{1} \mathbf{H}$ NMR (600 MHz, $\left.\mathbf{C D C l}_{3}\right) \delta 8.41(\mathrm{~d}, J=8.4 \mathrm{~Hz}, 1 \mathrm{H}), 7.83(\mathrm{~d}, J=7.9 \mathrm{~Hz}, 1 \mathrm{H}), 7.46-7.41(\mathrm{~m}$, $4 \mathrm{H}), 7.38(\mathrm{t}, J=7.6 \mathrm{~Hz}, 1 \mathrm{H}), 7.27(\mathrm{~d}, J=8.4 \mathrm{~Hz}, 2 \mathrm{H}), 7.21(\mathrm{~d}, J=8.2 \mathrm{~Hz}, 2 \mathrm{H})$, $7.06(\mathrm{~d}, J=8.2 \mathrm{~Hz}, 2 \mathrm{H}), 6.47(\mathrm{~d}, J=16.3 \mathrm{~Hz}, 1 \mathrm{H}), 3.73(\mathrm{~s}, 3 \mathrm{H}), 2.32(\mathrm{~s}, 3 \mathrm{H}), 1.41(\mathrm{~s}, 9 \mathrm{H}) ;{ }^{13} \mathbf{C}$ NMR (151 MHz, $\left.\mathbf{C D C l}_{3}\right) \delta 168.0,152.9,145.1,143.8,137.8,137.3,135.4,131.8,129.6,127.9,127.2,126.7,125.7$, 124.9, 124.7, 120.6, 118.4, 118.4, 116.4, 51.8, 35.1, 31.6, 21.8; IR (neat): $v_{\max }=29360,1735,1630,1447$, 1374, 1173, 1079, $568 \mathrm{~cm}^{-1}$; HRMS (EI) m/z: [M+] Calcd for $\mathrm{C}_{29} \mathrm{H}_{29} \mathrm{NO}_{4} \mathrm{~S}$ 487.1817, found 487.1818; $\boldsymbol{R}_{f} 0.60(\mathrm{Hex} / \mathrm{EtOAc}, 4 / 1)$. 


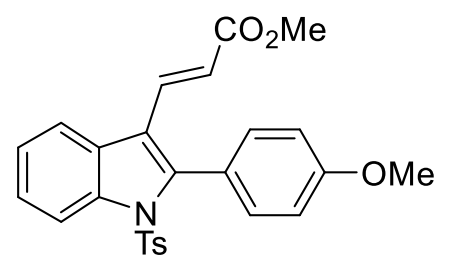

methyl(E)-3-(2-(4-methoxyphenyl)-1-tosyl-1H-indol-3-yl)acrylate, $2 \mathbf{j}$ : white solid (120 mg, 87\%); melting point 176-178 ${ }^{\circ} \mathrm{C} ;{ }^{1} \mathbf{H} \mathbf{N M R}\left(\mathbf{6 0 0} \mathbf{M H z}, \mathbf{C D C l}_{3}\right)$ $\delta 8.41(\mathrm{~d}, J=8.4 \mathrm{~Hz}, 1 \mathrm{H}), 7.81(\mathrm{~d}, J=7.9 \mathrm{~Hz}, 1 \mathrm{H}), 7.44(\mathrm{t}, J=7.8 \mathrm{~Hz}, 1 \mathrm{H})$, $7.40(\mathrm{~d}, J=16.3 \mathrm{~Hz}, 1 \mathrm{H}), 7.38(\mathrm{t}, J=7.6 \mathrm{~Hz}, 1 \mathrm{H}), 7.30(\mathrm{~d}, J=8.2 \mathrm{~Hz}, 2 \mathrm{H})$, $7.22(\mathrm{~d}, J=8.5 \mathrm{~Hz}, 2 \mathrm{H}), 7.08(\mathrm{~d}, J=8.2 \mathrm{~Hz}, 2 \mathrm{H}), 6.98(\mathrm{~d}, J=8.5 \mathrm{~Hz}, 2 \mathrm{H}), 6.44(\mathrm{~d}, J=16.3 \mathrm{~Hz}, 1 \mathrm{H}), 3.91$ (s, 3H), 3.72 (s, 3H), 2.31 (s, 3H); $\left.{ }^{13} \mathbf{C ~ N M R ~ ( 1 5 1 ~ M H z , ~} \mathbf{C D C l}_{3}\right) \delta$ 167.9, 160.7, 145.2, 143.5, 137.7, 137.1, 135.5, 133.5, 129.7, 127.9, 127.1, 125.7, 124.9, 121.9, 120.5, 118.4, 118.4, 116.5, 113.3, 55.5, 51.8, 21.9; IR (neat): $v_{\max }=2947,1735,1633,1448,1375,1175,1081,571 \mathrm{~cm}^{-1} ; \mathbf{H R M S}(\mathrm{EI}) \mathrm{m} / \mathrm{z}:\left[\mathrm{M}^{+}\right]$Calcd for $\mathrm{C}_{26} \mathrm{H}_{23} \mathrm{NO}_{5} \mathrm{~S} 461.1297$, found 461.1298; $\boldsymbol{R}_{f} 0.58$ (Hex/EtOAc, 4/1).

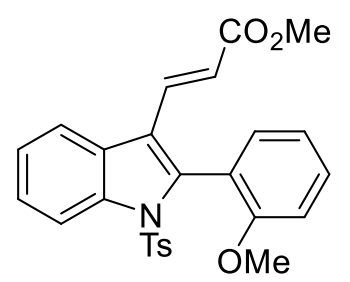

methyl (E)-3-(2-(2-methoxyphenyl)-1-tosyl-1H-indol-3-yl)acrylate, $2 \mathbf{k}$ : white solid (108 mg, 78\%); melting point $170-172{ }^{\circ} \mathrm{C} ;{ }^{1} \mathbf{H}$ NMR (600 $\left.\mathbf{~ M H z}, \mathbf{C D C l}_{3}\right) \delta 8.32$ $(\mathrm{d}, J=8.4 \mathrm{~Hz}, 1 \mathrm{H}), 7.85(\mathrm{~d}, J=7.8 \mathrm{~Hz}, 1 \mathrm{H}), 7.50(\mathrm{td}, J=8.1,1.8 \mathrm{~Hz}, 1 \mathrm{H}), 7.44-$ 7.40 (m, 3H), 7.40-7.34 (m, 2H), $7.12(\mathrm{~d}, J=8.1 \mathrm{~Hz}, 2 \mathrm{H}), 7.08(\mathrm{dd}, J=7.4,1.7 \mathrm{~Hz}$, 1H), $7.03(\mathrm{t}, J=7.4 \mathrm{~Hz}, 1 \mathrm{H}), 6.97(\mathrm{~d}, J=8.3 \mathrm{~Hz}, 1 \mathrm{H}), 6.42(\mathrm{~d}, J=16.3 \mathrm{~Hz}, 1 \mathrm{H})$, 3.71 (s, 3H), 3.69 (s, 3H), 2.33 (s, 3H); ${ }^{13} \mathbf{C}$ NMR (151 MHz, $\left.\mathbf{C D C l}_{3}\right) \delta$ 168.0, 158.9, 145.0, 140.3, 137.3, 137.1, 136.0, 133.1, 131.8, 129.6, 127.8, 127.3, 125.4, 124.4, 120.6, 120.1, 119.1, 118.0, 117.9, 115.7, 111.0, 55.6, 51.7, 21.8; IR (neat): $v_{\max }=2947,1738,1632,1435,1371,1173,1091,569 \mathrm{~cm}^{-1}$; HRMS (EI) m/z: $\left[\mathrm{M}^{+}\right]$Calcd for $\mathrm{C}_{26} \mathrm{H}_{23} \mathrm{NO}_{5} \mathrm{~S} 461.1297$, found 461.1299; $\boldsymbol{R}_{\boldsymbol{f}} 0.53$ (Hex/EtOAc, 4/1).

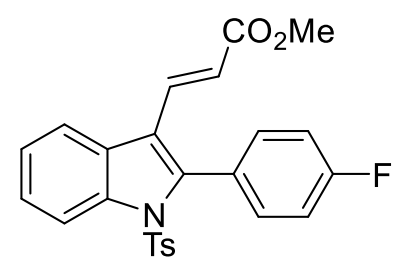

methyl (E)-3-(2-(4-fluorophenyl)-1-tosyl-1H-indol-3-yl)acrylate, 2l: white solid (80 mg, 59\%); melting point $136-138^{\circ} \mathrm{C} ;{ }^{1} \mathbf{H}$ NMR (600 $\left.\mathbf{M H z}, \mathbf{C D C l}_{3}\right) \delta$ $8.41(\mathrm{~d}, J=8.2, \mathrm{~Hz}, 1 \mathrm{H}), 7.82(\mathrm{~d}, J=7.7 \mathrm{~Hz}, 1 \mathrm{H}), 7.46(\mathrm{t}, J=8.2 \mathrm{~Hz}, 1 \mathrm{H}), 7.39$ (t, $J=7.7 \mathrm{~Hz}, 1 \mathrm{H}), 7.34(\mathrm{~d}, J=16.3 \mathrm{~Hz}, 1 \mathrm{H}), 7.30(\mathrm{~d}, J=8.2 \mathrm{~Hz}, 2 \mathrm{H}), 7.27$ $(\mathrm{ddd}, J=8.6,5.5,2.8 \mathrm{~Hz}, 2 \mathrm{H}), 7.15(\mathrm{t}, J=8.6 \mathrm{~Hz}, 2 \mathrm{H}), 7.10(\mathrm{~d}, J=8.2 \mathrm{~Hz}, 2 \mathrm{H}), 6.45(\mathrm{~d}, J=16.3 \mathrm{~Hz}, 1 \mathrm{H})$, $3.72(\mathrm{~s}, 3 \mathrm{H}), 2.32(\mathrm{~s}, 3 \mathrm{H}) ;{ }^{13} \mathbf{C}$ NMR (151 MHz, $\left.\mathbf{C D C l}_{3}\right) \delta 167.7,163.7(\mathrm{~d}, J=250.4 \mathrm{~Hz}), 145.5,141.9$, 137.6, 136.4, 135.4, 133.9 (d, $J=8.5 \mathrm{~Hz}), 129.8,127.7,127.0,126.0,125.9$ (d, $J=3.4 \mathrm{~Hz}), 125.0,120.6$, 119.1, 118.8, 116.4, 115.1 (d, $J=21.9 \mathrm{~Hz}$ ), 51.8, 21.8; IR (neat): $v_{\max }=2947,1738,1633,1447,1367$, 1175, 1081, 977, $570 \mathrm{~cm}^{-1}$; HRMS (EI) m/z: [M+] Calcd for $\mathrm{C}_{25} \mathrm{H}_{20} \mathrm{FNO}_{4} \mathrm{~S} 449.1097$, found 449.1095; $\boldsymbol{R}_{f} 0.59(\mathrm{Hex} / \mathrm{EtOAc}, 4 / 1)$. 


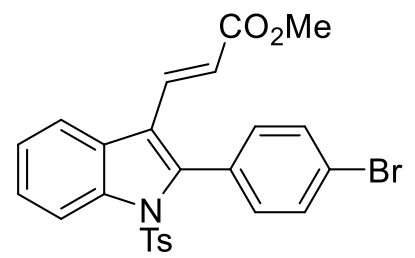

methyl $(E)$-3-(2-(4-bromophenyl)-1-tosyl-1H-indol-3-yl)acrylate, $2 \mathbf{m}$ : white so lid (135 mg, 88\%); melting point 161-163 ${ }^{\circ} \mathrm{C} ;{ }^{1} \mathbf{H}$ NMR (600 MHz, $\left.\mathbf{C D C l}_{3}\right) \delta$ $8.40(\mathrm{~d}, J=8.4 \mathrm{~Hz}, 1 \mathrm{H}), 7.82(\mathrm{~d}, J=7.8 \mathrm{~Hz}, 1 \mathrm{H}), 7.60(\mathrm{~d}, J=8.2 \mathrm{~Hz}, 2 \mathrm{H}), 7.47$ $(\mathrm{td}, J=7.9,1.14 \mathrm{~Hz}, 1 \mathrm{H}), 7.39(\operatorname{td} J=7.5,0.8 \mathrm{~Hz}, 1 \mathrm{H}), 7.33(\mathrm{~d}, J=16.3 \mathrm{~Hz}$, $1 \mathrm{H}), 7.31(\mathrm{~d}, J=8.5 \mathrm{~Hz}, 2 \mathrm{H}), 7.18(\mathrm{~d}, J=8.5 \mathrm{~Hz}, 2 \mathrm{H}), 7.11(\mathrm{~d}, J=8.2 \mathrm{~Hz}, 2 \mathrm{H}), 6.46(\mathrm{~d}, J=16.3 \mathrm{~Hz}, 1 \mathrm{H})$, 3.73 (s, 3H), 2.33 (s, 3H); ${ }^{13}$ C NMR (151 MHz, CDCl $\left.\mathbf{~}_{3}\right) \delta$ 167.6, 145.5, 141.7, 137.7, 136.3, 135.3, 133.5, 131.2, 130.9, 129.8, 129.5, 127.0, 126.1, 125.1, 124.5, 120.7, 119.4, 118.0, 116.4, 51.9, 21.8; IR (neat): $v_{\max }=2948,1738,1435,1366,1175,1091,835,666,570 \mathrm{~cm}^{-1} ; \mathbf{H R M S}(\mathrm{EI}) \mathrm{m} / \mathrm{z}:\left[\mathrm{M}^{+}\right]$Calcd for $\mathrm{C}_{25} \mathrm{H}_{20} \mathrm{BrNO}_{4} \mathrm{~S} 509.0296$, found 509.0299; $\boldsymbol{R}_{f} 0.60$ (Hex/EtOAc, 4/1).

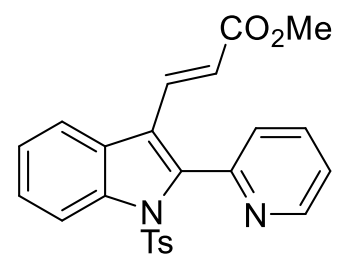

methyl (E)-3-(2-(pyridin-2-yl)-1-tosyl-1H-indol-3-yl)acrylate, 2n: yellow solid (56 mg, 43\%); melting point $180-182{ }^{\circ} \mathrm{C} ;{ }^{1} \mathbf{H}$ NMR (600 $\left.\mathbf{M H z}, \mathbf{C D C l}_{3}\right) \delta 8.79(\mathrm{~d}, J=$ $4.3 \mathrm{~Hz}, 1 \mathrm{H}), 8.23(\mathrm{~d}, J=8.4 \mathrm{~Hz}, 2 \mathrm{H}), 7.86(\mathrm{td}, J=7.8,1.7 \mathrm{~Hz}, 1 \mathrm{H}), 7.83(\mathrm{~d}, J=8.0$ $\mathrm{Hz}, 1 \mathrm{H}), 7.68(\mathrm{~d}, J=8.4 \mathrm{~Hz}, 2 \mathrm{H}), 7.62(\mathrm{~d}, J=7.8 \mathrm{~Hz}, 1 \mathrm{H}), 7.50(\mathrm{~d}, J=16.3 \mathrm{~Hz}$, 1H), 7.46-7.41 (m, 2H), $7.36(\mathrm{t}, J=8.0 \mathrm{~Hz}, 1 \mathrm{H}), 7.17(\mathrm{~d}, J=8.0 \mathrm{~Hz}, 1 \mathrm{H}), 6.45(\mathrm{~d}, J=16.3 \mathrm{~Hz}, 1 \mathrm{H}), 3.72$ (s, 3H), $2.31(\mathrm{~s}, 3 \mathrm{H}) ;{ }^{13} \mathbf{C}$ NMR (151 MHz, $\left.\mathbf{C D C l}_{3}\right) \delta$ 167.6, 150.3, 149.3, 145.4, 141.2, 137.1, 136.2, 135.0, 134.7, 129.9, 127.9, 127.9, 127.5, 126.2, 125.0, 124.1, 121.1, 119.8, 119.7, 115.0, 51.8, 21.8; IR (neat): $v_{\max }=2947,1738,1634,1434,1367,1177,1086,573 \mathrm{~cm}^{-1}$; HRMS (EI) m/z: [M+] Calcd for $\mathrm{C}_{24} \mathrm{H}_{20} \mathrm{~N}_{2} \mathrm{O}_{4} \mathrm{~S}$ 432.1144, found 432.1147; $\boldsymbol{R}_{f} 0.51$ (Hex/EtOAc, 7/3).

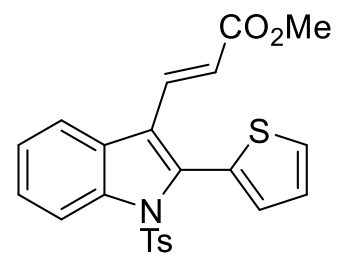

methyl (E)-3-(2-(thiophen-2-yl)-1-tosyl-1H-indol-3-yl)acrylate, 2o: yellow solid (118 mg, 90\%); melting point 166-168 ${ }^{\circ} \mathrm{C} ;{ }^{1} \mathbf{H}$ NMR (600 MHz, $\left.\mathbf{C D C l}_{3}\right) \delta 8.40(\mathrm{~d}$, $J=8.5 \mathrm{~Hz}, 1 \mathrm{H}), 7.82(\mathrm{~d}, J=7.9 \mathrm{~Hz}, 1 \mathrm{H}), 7.59(\mathrm{dd}, J=5.1,1.2 \mathrm{~Hz}, 1 \mathrm{H}), 7.51(\mathrm{~d}, J$ $=16.3 \mathrm{~Hz}, 1 \mathrm{H}), 7.46(\mathrm{ddd}, J=8.5,7.5,1.2 \mathrm{~Hz}, 1 \mathrm{H}), 7.40(\mathrm{~d}, J=8.4 \mathrm{~Hz}, 2 \mathrm{H}), 7.39$ $7.35(\mathrm{~m}, 1 \mathrm{H}), 7.16(\mathrm{dd}, J=5.1,3.6 \mathrm{~Hz}, 1 \mathrm{H}), 7.13-7.10(\mathrm{~m}, 3 \mathrm{H}), 6.45(\mathrm{~d}, J=16.3 \mathrm{~Hz}, 1 \mathrm{H}), 3.74(\mathrm{~s}, 3 \mathrm{H})$, 2.33 (s, 3H); ${ }^{13} \mathbf{C}$ NMR (151 MHz, CDCl $) \delta$ 167.7, 145.4, 137.9, 136.4, 135.1, 132.9, 129.9, 129.8, 129.5, $129.4,127.4,127.1,126.9,126.2,124.9,120.6,120.5,119.4,116.2,51.9,21.8$; IR (neat): $v_{\max }=2949$, 1734, 1631, 1448, 1171, 1089, $568 \mathrm{~cm}^{-1}$; HRMS (EI) m/z: [M+] Calcd for $\mathrm{C}_{23} \mathrm{H}_{19} \mathrm{NO}_{4} \mathrm{~S}_{2} 437.0755$, found 437.0757; $\boldsymbol{R}_{f} 0.52$ (Hex/EtOAc, 7/3). 


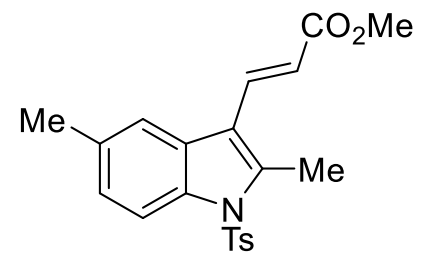

methyl (E)-3-(2,5-dimethyl-1-tosyl-1H-indol-3-yl)acrylate, 4a: white solid (92 mg, 80\%); melting point $178-180{ }^{\circ} \mathrm{C} ;{ }^{1} \mathbf{H}$ NMR (600 MHz, $\left.\mathbf{C D C l}_{3}\right) \delta 8.12$ $(\mathrm{d}, J=8.6 \mathrm{~Hz}, 1 \mathrm{H}), 7.82(\mathrm{~d}, J=16.1 \mathrm{~Hz}, 1 \mathrm{H}), 7.66(\mathrm{~d}, J=8.3 \mathrm{~Hz}, 2 \mathrm{H}), 7.54$ (s, 1H), $7.21(\mathrm{~d}, J=8.3 \mathrm{~Hz}, 2 \mathrm{H}), 7.15(\mathrm{~d}, J=8.6 \mathrm{~Hz}, 1 \mathrm{H}), 6.49$ (d, $J=16.1$ Hz, 1H), 3.81 (s, 3H), 2.70 (s, 3H), 2.44 (s, 3H), 2.34 (s, 3H); ${ }^{13}$ C NMR (151 MHz, CDCl $) \delta$ 168.0, 145.4, 140.1, 136.2, 135.0, 134.1, 130.2, 127.8, 126.6, 126.3, 120.1, 117.9, 115.9, 114.6, 51.9, 21.8, 21.6, 13.4 (one carbon peak is overlapping); IR (neat): $v_{\max }=2949,1738,1638,1435,1365,1169,1092,569 \mathrm{~cm}^{-1}$; HRMS (EI) m/z: $\left[\mathrm{M}^{+}\right]$Calcd for $\mathrm{C}_{21} \mathrm{H}_{21} \mathrm{NO}_{4} \mathrm{~S} 383.1191$, found 383.1194; $\boldsymbol{R}_{f} 0.60$ (Hex/EtOAc, 4/1).

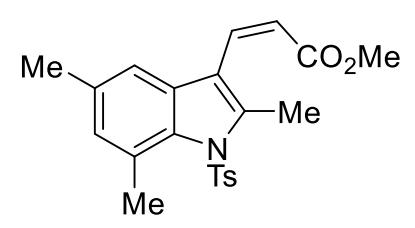

methyl (Z)-3-(2,5,7-trimethyl-1-tosyl-1H-indol-3-yl)acrylate 4b: white solid (13 mg, 35\%, at $0.09 \mathrm{mmol}$ scale of $Z$-isomer); melting point $86-88{ }^{\circ} \mathrm{C}$; ${ }^{1} \mathbf{H}$ NMR (600 MHz, $\left.\mathbf{C D C l}_{3}\right) \delta 7.40(\mathrm{~d}, J=8.4 \mathrm{~Hz}, 2 \mathrm{H}), 7.12(\mathrm{~d}, J=8.4 \mathrm{~Hz}, 2 \mathrm{H})$, $6.88(\mathrm{~s}, 1 \mathrm{H}), 6.78(\mathrm{~d}, J=12.0 \mathrm{~Hz}, 1 \mathrm{H}), 6.76(\mathrm{~s}, 1 \mathrm{H}), 6.13(\mathrm{~d}, J=12.0 \mathrm{~Hz}, 1 \mathrm{H})$, 3.55 (s, 3H), 2.64 (s, 3H), 2.43 (s, 3H), 2.31 (s, 6H); ${ }^{13}$ C NMR (151 MHz, CDCl $) \delta$ 166.0, 144.3, 139.4, 136.5, 135.8, 134.5, 134.4, 132.9, 130.1, 129.5, 127.0, 126.6, 123.6, 121.3, 117.1, 29.9, 22.5, 21.5, 21.2, 16.1; IR (neat): $v_{\max }=2921,1727,1637,1439,1365,1173,1066,812,534 \mathrm{~cm}^{-1} ; \mathbf{H R M S}(\mathrm{EI}) \mathrm{m} / \mathrm{z}:\left[\mathrm{M}^{+}\right]$ Calcd for $\mathrm{C}_{22} \mathrm{H}_{23} \mathrm{NO}_{4} \mathrm{~S} 397.1348$, found 397.1350; $\boldsymbol{R}_{f} 0.60$ (Hex/EtOAc, 4/1).

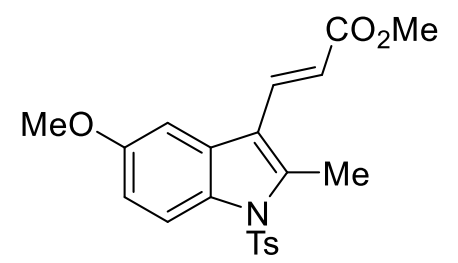

methyl $(E)-3-(5-m e t h o x y-2-m e t h y l-1-t o s y l-1 H$-indol-3-yl)acrylate, 4c:white solid (111 mg, 93\%); melting point $144-146{ }^{\circ} \mathrm{C} ;{ }^{1} \mathbf{H}$ NMR (600 MHz, $\left.\mathbf{C D C l}_{3}\right)$ $\delta 8.15(\mathrm{~d}, J=9.1 \mathrm{~Hz}, 1 \mathrm{H}), 7.81(\mathrm{~d}, J=16.1 \mathrm{~Hz}, 1 \mathrm{H}), 7.65(\mathrm{~d}, J=8.4 \mathrm{~Hz}, 2 \mathrm{H})$, $7.22(\mathrm{~d}, J=8.4 \mathrm{~Hz}, 2 \mathrm{H}), 7.18(\mathrm{~d}, J=2.5 \mathrm{~Hz}, 1 \mathrm{H}), 6.94(\mathrm{dd}, J=9.1,2.5 \mathrm{~Hz}$, $1 \mathrm{H}), 6.42(\mathrm{~d}, J=16.1 \mathrm{~Hz}, 1 \mathrm{H}), 3.86(\mathrm{~s}, 3 \mathrm{H}), 3.81(\mathrm{~s}, 3 \mathrm{H}), 2.70(\mathrm{~s}, 3 \mathrm{H}), 2.35$ (s, 3H); ${ }^{13} \mathbf{C}$ NMR (151 MHz, CDCl $\left._{3}\right)$ 168.0, 157.2, 145.4, 140.6, 136.1, 131.4, 130.2, 128.7, 126.6, 117.9, 116.1, 115.8, 113.0, 103.4, 55.0, 51.9, 21.8, 13.5; IR (neat): $v_{\max }=2948,1738,1636,1432,1365,1168,1092,563 \mathrm{~cm}^{-1}$; HRMS (EI) $\mathrm{m} / \mathrm{z}:\left[\mathrm{M}^{+}\right]$Calcd for $\mathrm{C}_{21} \mathrm{H}_{21} \mathrm{NO}_{5} \mathrm{~S} 399.1140$, found 399.1138; $\boldsymbol{R}_{\boldsymbol{f}} 0.58$ (Hex/EtOAc, 4/1).

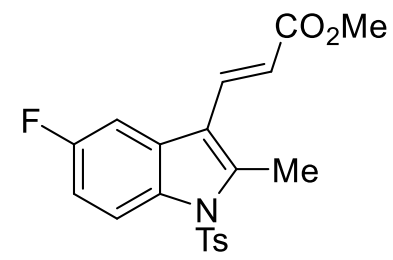

methyl(E)-3-(5-fluoro-2-methyl-1-tosyl-1H-indol-3-yl)acrylate, $4 \mathbf{d}$ : white solid (101 mg, 87\%); melting point $130-132{ }^{\circ} \mathrm{C} ;{ }^{1} \mathbf{H}$ NMR (600 MHz, $\left.\mathbf{C D C l}_{3}\right) \delta 8.21$ $\left(\mathrm{dd}, J=9.2 \mathrm{~Hz},{ }^{4} J_{H-F}=4.6 \mathrm{~Hz}, 1 \mathrm{H}\right), 7.78(\mathrm{~d}, J=16.1 \mathrm{~Hz}, 1 \mathrm{H}), 7.66(\mathrm{~d}, J=8.4$ $\mathrm{Hz}, 2 \mathrm{H}), 7.40\left(\mathrm{dd},{ }^{3} J_{H-F}=9.1 \mathrm{~Hz}, J=2.5 \mathrm{~Hz}, 1 \mathrm{H}\right), 7.24(\mathrm{~d}, J=8.4 \mathrm{~Hz}, 2 \mathrm{H}), 7.06$ (ddd, $\left.J=9.2,2.5 \mathrm{~Hz},{ }^{3} J_{H-F}=9.0 \mathrm{~Hz}, 1 \mathrm{H}\right), 6.39(\mathrm{~d}, J=16.1 \mathrm{~Hz}, 1 \mathrm{H}), 3.81(\mathrm{~s}, 3 \mathrm{H}), 2.70(\mathrm{~s}, 3 \mathrm{H}), 2.36(\mathrm{~s}, 3 \mathrm{H})$; ${ }^{13}$ C NMR (151 MHz, CDCl $) \delta 167.8,160.4(\mathrm{~d}, J=241.4$ Hz), 145.7, 141.4, 135.9, 135.5, 133.1, 130.3, $128.6(\mathrm{~d}, J=9.8 \mathrm{~Hz}), 126.7,118.4,116.0(\mathrm{~d}, J=9.2 \mathrm{~Hz}), 115.9(\mathrm{~d}, J=3.7 \mathrm{~Hz}), 112.7(\mathrm{~d}, J=24.8 \mathrm{~Hz})$, 
$106.0(\mathrm{~d}, J=24.9 \mathrm{~Hz}), 51.0,21.8,13.5 ; \quad$ IR (neat): $v_{\max }=2949,1735,1633,1435,1177,1091,939,565$ $\mathrm{cm}^{-1}$; HRMS (EI) m/z: $\left[\mathrm{M}^{+}\right]$Calcd for $\mathrm{C}_{20} \mathrm{H}_{18} \mathrm{FNO}_{4} \mathrm{~S} 387.0941$, found 387.0938; $\boldsymbol{R}_{\boldsymbol{f}} 0.60$ (Hex/EtOAc, 4/1).

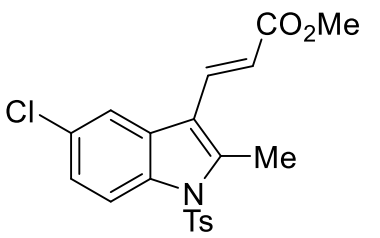

methyl (E)-3-(5-chloro-2-methyl-1-tosyl-1H-indol-3-yl)acrylate, 4e: white solid (95 mg, 78\%); melting point 182-184 ${ }^{\circ} \mathrm{C} ;{ }^{1} \mathbf{H}$ NMR (600 MHz, CDCl $) \delta 8.19$ $(\mathrm{d}, J=8.9 \mathrm{~Hz}, 1 \mathrm{H}), 7.77(\mathrm{~d}, J=16.1 \mathrm{~Hz}, 1 \mathrm{H}), 7.71(\mathrm{~d}, J=1.9 \mathrm{~Hz}, 1 \mathrm{H}), 7.66(\mathrm{~d}$, $J=8.4 \mathrm{~Hz}, 2 \mathrm{H}), 7.30(\mathrm{dd}, J=8.9,1.9 \mathrm{~Hz}, 1 \mathrm{H}), 7.24(\mathrm{~d}, J=8.4 \mathrm{~Hz}, 2 \mathrm{H}), 6.42(\mathrm{~d}$, $J=16.1 \mathrm{~Hz}, 1 \mathrm{H}), 3.82$ (s, 3H), 2.70 (s, 3H), 2.37 (s, 3H); ${ }^{13} \mathbf{C}$ NMR (151 MHz, CDCl $\left.{ }_{3}\right) \delta$ 167.6, 145.8, 141.1, 135.8, 135.3, 135.2, 130.4, 128.8, 126.7, 125.1, 119.7, 118.6, 115.9, 115.5, 51.0, 21.8, 13.5; IR (neat): $v_{\max }=2946,1738,1634,1432,1372,1170,1044,669,634,588 \mathrm{~cm}^{-1} ;$ HRMS (EI) m/z: [M+] Calcd for $\mathrm{C}_{20} \mathrm{H}_{18} \mathrm{ClNO}_{4} \mathrm{~S}$ 403.0645, found 403.0648; $\boldsymbol{R}_{\boldsymbol{f}} 0.60$ (Hex/EtOAc, 4/1).

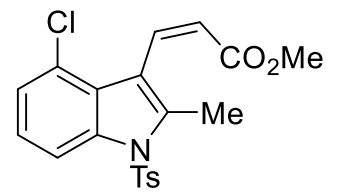

methyl (Z)-3-(4-chloro-2-methyl-1-tosyl-1H-indol-3-yl)acrylate, 4f: viscous oil (27 mg, $66 \%$ at $0.1 \mathrm{mmol}$ scale of $Z$-isomer); ${ }^{1} \mathbf{H}$ NMR (600 $\left.\mathbf{M H z}, \mathbf{C D C l}_{3}\right) \delta 8.07$ (dd, $J=7.9,1.2 \mathrm{~Hz}, 1 \mathrm{H}), 7.68(\mathrm{~d}, J=8.3 \mathrm{~Hz}, 2 \mathrm{H}), 7.33(\mathrm{~d}, J=11.9 \mathrm{~Hz}, 1 \mathrm{H}), 7.23(\mathrm{~d}, J$ $=8.3 \mathrm{~Hz}, 2 \mathrm{H}), 7.18-7.12(\mathrm{~m}, 2 \mathrm{H}), 6.19(\mathrm{~d}, J=11.9 \mathrm{~Hz}, 1 \mathrm{H}), 3.57(\mathrm{~s}, 3 \mathrm{H}), 2.47(\mathrm{~s}, 3 \mathrm{H}), 2.35(\mathrm{~s}, 3 \mathrm{H}) ;{ }^{13} \mathbf{C}$ NMR (151 MHz, $\left.\mathbf{C D C l}_{3}\right) \delta 165.9,145.2,137.4,137.0,136.8,136.1,130.1,126.7,125.9,124.8,124.8$, 123.1, 116.3, 113.3, 51.5, 21.8, 14.4 (One carbon peak is overlaps); IR (neat): $v_{\max }=2950,1724,1633$, 1426, 1369, 1169, 1089, 988, 665, 577, $534 \mathrm{~cm}^{-1}$; HRMS (EI) m/z: [M+] Calcd for $\mathrm{C}_{20} \mathrm{H}_{18} \mathrm{ClNO}_{4} \mathrm{~S}$ 403.0645, found 403.0645; $\boldsymbol{R}_{f} 0.52$ (Hex/EtOAc, 4/1).

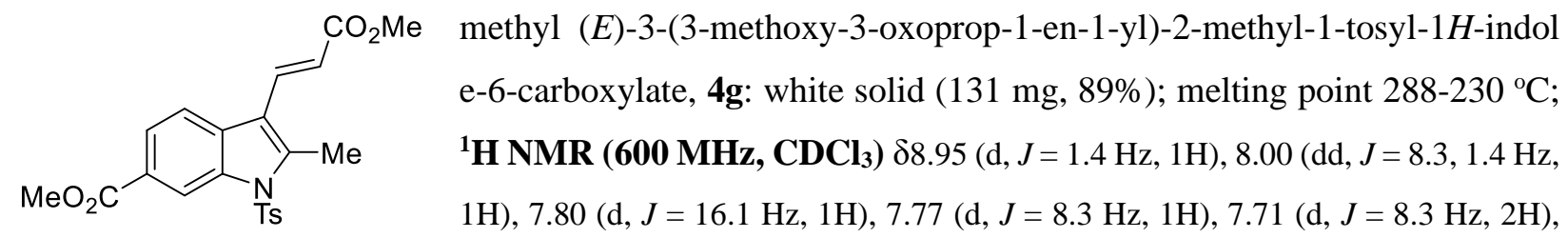
$7.25(\mathrm{~d}, J=8.3 \mathrm{~Hz}, 2 \mathrm{H}), 6.48(\mathrm{~d}, J=16.1 \mathrm{~Hz}, 1 \mathrm{H}), 3.97(\mathrm{~s}, 3 \mathrm{H}), 3.81(\mathrm{~s}, 3 \mathrm{H}), 2.74(\mathrm{~s}, 3 \mathrm{H}), 2.36(\mathrm{~s}, 3 \mathrm{H})$; ${ }^{\mathbf{1 3}} \mathbf{C} \mathbf{~ N M R}$ (151 MHz, $\mathbf{C D C l}_{3}$ ) $\delta 168.6,167.3,145.9,142.1,137.2,135.9,135.4,132.0,130.4,126.8,126.8,125.6,119.6,118.0$, 116.5, 115.9, 53.0, 51.0, 21.3, 13.6.; IR (neat): $v_{\max }=2950,1724,1633,1426,1169,1089,988,665,577$, $534 \mathrm{~cm}^{-1}$; HRMS (EI) m/z: $\left[\mathrm{M}^{+}\right]$Calcd for for $\mathrm{C}_{22} \mathrm{H}_{21} \mathrm{NO}_{6} \mathrm{~S} 427.1090$, found 427.1092; $\boldsymbol{R}_{f} 0.59$ (Hex/EtOAc, 4/1). 


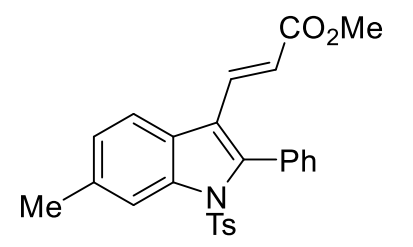

methyl $(E)$-3-(6-methyl-2-phenyl-1-tosyl-1H-indol-3-yl)acrylate, 4 h: white soli d (106 mg, 79\%); melting point 186-188 ${ }^{\circ} \mathrm{C} ;{ }^{1} \mathbf{H}$ NMR (600 $\left.\mathbf{M H z}, \mathbf{C D C l}_{3}\right) \delta$ $8.23(\mathrm{~s}, 1 \mathrm{H}), 7.70(\mathrm{~d}, J=8.1 \mathrm{~Hz}, 1 \mathrm{H}), 7.50(\mathrm{t}, J=7.4 \mathrm{~Hz}, 1 \mathrm{H}), 7.44(\mathrm{t}, J=7.4$ $\mathrm{Hz}, 2 \mathrm{H}), 7.36(\mathrm{~d}, J=16.3 \mathrm{~Hz}, 1 \mathrm{H}), 7.30(\mathrm{~d}, J=8.4 \mathrm{~Hz}, 2 \mathrm{H}), 7.28(\mathrm{~d}, J=8.0 \mathrm{~Hz}$, 2H), $7.21(\mathrm{~d}, J=8.4 \mathrm{~Hz}, 1 \mathrm{H}), 7.09(\mathrm{~d}, J=8.1 \mathrm{~Hz}, 2 \mathrm{H}), 6.43(\mathrm{~d}, J=16.3 \mathrm{~Hz}, 1 \mathrm{H}), 3.71(\mathrm{~s}, 3 \mathrm{H}), 2.56(\mathrm{~s}, 3 \mathrm{H})$, 2.32 (s, 3H); ${ }^{13} \mathbf{C}$ NMR (151 MHz, CDCl $) \delta 167.8,145.2,142.8,138.1,137.0,136.1,135.6,132.0,130.1$, 129.7, 129.7, 127.8, 127.1, 126.4, 125.6, 120.2, 118.6, 118.6, 116.5, 51.8, 22.3, 21.8; IR (neat): $v_{\max }=$ 2949, 1738, 1631, 1439, 1375, 1175, 1083, $579 \mathrm{~cm}^{-1}$; HRMS (EI) m/z: [M+] Calcd for $\mathrm{C}_{26} \mathrm{H}_{23} \mathrm{NO}_{4} \mathrm{~S}$ 445.1348, found 445.1346; $\boldsymbol{R}_{f} 0.59$ (Hex/EtOAc, 4/1).

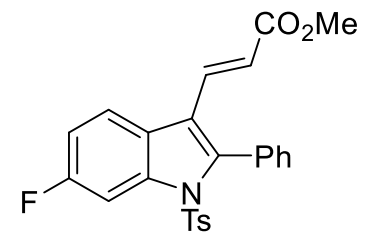

methyl (E)-3-(6-fluoro-2-phenyl-1-tosyl-1H-indol-3-yl)acrylate, 4i: white solid (85 mg, 63\%); melting point $178-180{ }^{\circ} \mathrm{C} ;{ }^{1} \mathbf{H}$ NMR (600 MHz, $\left.\mathbf{C D C l}_{3}\right) \delta 8.16$ $(\mathrm{dd}, J=10.2,2.4 \mathrm{~Hz}, 1 \mathrm{H}), 7.76(\mathrm{dd}, J=8.8,5.3 \mathrm{~Hz}, 1 \mathrm{H}), 7.51(\mathrm{t}, J=7.5 \mathrm{~Hz}, 1 \mathrm{H})$, $7.45(\mathrm{t}, J=7.5 \mathrm{~Hz}, 2 \mathrm{H}), 7.34(\mathrm{~d}, J=16.3 \mathrm{~Hz}, 1 \mathrm{H}), 7.30(\mathrm{~d}, J=8.4 \mathrm{~Hz}, 2 \mathrm{H}), 7.27$ $(\mathrm{d}, J=8.4 \mathrm{~Hz}, 2 \mathrm{H}), 7.14(\mathrm{td}, J=8.8,2.4 \mathrm{~Hz}, 1 \mathrm{H}), 7.11(\mathrm{~d}, J=8.1 \mathrm{~Hz}, 2 \mathrm{H}), 6.39(\mathrm{~d}, J=16.3 \mathrm{~Hz}, 1 \mathrm{H}), 3.71$

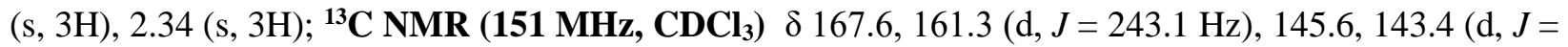
$3.6 \mathrm{~Hz}), 137.0(\mathrm{~d}, J=12.4 \mathrm{~Hz}), 136.5,135.2,132.0,129.9,129.8,129.6,127.9,127.2,124.1(\mathrm{~d}, J=1.5$ $\mathrm{Hz}), 121.4(\mathrm{~d}, J=9.7 \mathrm{~Hz}), 118.9,118.1,113.1(\mathrm{~d}, J=23.9 \mathrm{~Hz}), 103.8(\mathrm{~d}, J=28.9 \mathrm{~Hz}), 51.8,21.8$; IR (neat): $v_{\max }=2954,1737,1636,1446,1372,1174,1097,938,847,583 \mathrm{~cm}^{-1}$; HRMS (EI) m/z: [M+ ${ }^{+}$Calcd for $\mathrm{C}_{25} \mathrm{H}_{20} \mathrm{FNO}_{4} \mathrm{~S} 449.1097$, found 449.1097; $\boldsymbol{R}_{f} 0.58$ (Hex/EtOAc, 8/1).

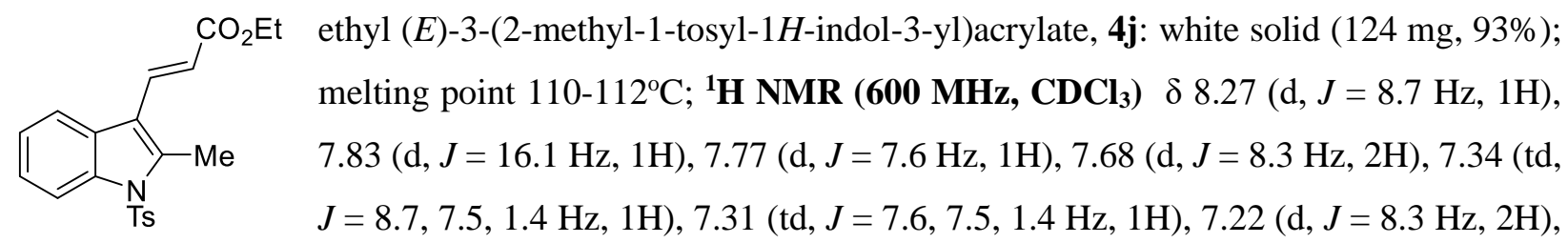
$6.49(\mathrm{~d}, J=16.1 \mathrm{~Hz}, 1 \mathrm{H}), 4.27(\mathrm{q}, J=7.1 \mathrm{~Hz}, 2 \mathrm{H}), 2.72(\mathrm{~s}, 3 \mathrm{H}), 2.35(\mathrm{~s}, 3 \mathrm{H}), 1.34(\mathrm{t}, J=7.1 \mathrm{~Hz}, 3 \mathrm{H}) ;{ }^{13} \mathbf{C}$ NMR (151 MHz, $\left.\mathbf{C D C l}_{3}\right) \delta 167.6,144.6,139.8,136.9,136.2,135.7,130.7,127.6,126.7,125.0,124.4$, 120.0, 118.7, 116.1, 114.9, 59.7, 21.8, 14.6, 13.4; IR (neat): $v_{\max }=2980,1708,1630,1453,1367,1281$, 1174, 1089, 995, 660, $570 \mathrm{~cm}^{-1}$; HRMS (EI) m/z: [M+] Calcd for $\mathrm{C}_{21} \mathrm{H}_{21} \mathrm{NO}_{4} \mathrm{~S}$ 383.1191, found 383.1192; $\boldsymbol{R}_{f} 0.58(\mathrm{Hex} / \mathrm{EtOAc}, 8 / 1)$ 


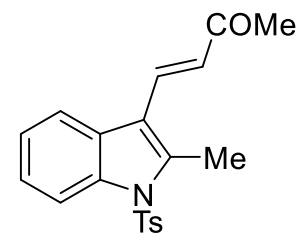

(E)-4-(2-methyl-1-tosyl-1H-indol-3-yl)but-3-en-2-one, 4k: white solid (111 mg, $89 \%)$; melting point $122-124{ }^{\circ} \mathrm{C} ;{ }^{1} \mathbf{H}$ NMR (600 $\left.\mathbf{M H z}, \mathbf{C D C l}_{3}\right) \delta 8.27(\mathrm{~d}, J=8.6 \mathrm{~Hz}$, $1 \mathrm{H}), 7.78(\mathrm{~d}, J=7.6 \mathrm{~Hz}, 1 \mathrm{H}), 7.71(\mathrm{~d}, J=8.3 \mathrm{~Hz}, 2 \mathrm{H}), 7.69(\mathrm{~d}, J=16.2 \mathrm{~Hz}, 1 \mathrm{H}), 7.35$ $(\mathrm{td}, J=8.6,7.8,1.4 \mathrm{~Hz}, 1 \mathrm{H}), 7.32(\mathrm{td}, J=7.6,7.3,1.4 \mathrm{~Hz}, 1 \mathrm{H}), 7.23(\mathrm{~d}, J=8.3 \mathrm{~Hz}$, 2H), $6.82(\mathrm{~d}, J=16.2 \mathrm{~Hz}, 1 \mathrm{H}), 2.73(\mathrm{~s}, 3 \mathrm{H}), 2.37(\mathrm{~s}, 3 \mathrm{H}), 2.35(\mathrm{~s}, 3 \mathrm{H}) ;{ }^{13} \mathbf{C}$ NMR $\left(\mathbf{1 5 1} \mathbf{~ M H z}, \mathbf{C D C l}_{3}\right) \delta$ 197.0, 145.0, 142.5, 135.8, 135.5, 134.0, 130.8, 130.0, 130.0, 129.0, 128.3, 125.2, 109.6, 92.0, 75.4, 28.1, 21.8, 4.6; IR (neat): $v_{\max }=2952,1660,1596,1452,1364,1174,1087,811,691,571,539 \mathrm{~cm}^{-1}$; HRMS (EI) m/z: $\left[\mathrm{M}^{+}\right]$Calcd for $\mathrm{C}_{20} \mathrm{H}_{19} \mathrm{NO}_{3} \mathrm{~S} 353.1086$, found 353.1083; $\boldsymbol{R}_{\boldsymbol{f}} 0.58$ (Hex/EtOAc, 8/1)

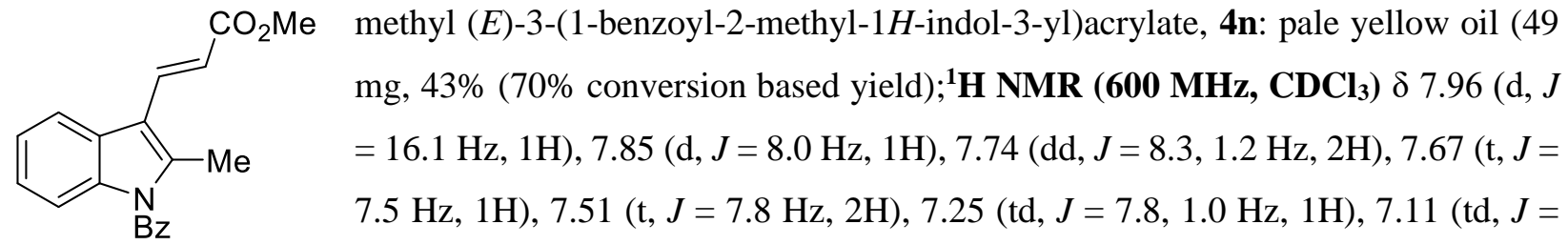
7.8, $1.0 \mathrm{~Hz}, 1 \mathrm{H}), 7.04(\mathrm{~d}, J=8.3 \mathrm{~Hz}, 1 \mathrm{H}), 6.57(\mathrm{~d}, J=16.1 \mathrm{~Hz}, 1 \mathrm{H}), 3.84(\mathrm{~s}, 3 \mathrm{H}), 2.52(\mathrm{~s}, 3 \mathrm{H}) ;{ }^{13} \mathbf{C ~ N M R}$ (151 MHz, $\left.\mathbf{C D C l}_{3}\right) \delta 169.7,168.4,140.9,137.2,136.6,134.7,133.0,130.3,129.2,127.2,124.0,123.6$, 120.0, 116.9, 115.1, 114.4, 51.8, 13.6; IR (neat): $v_{\max }=2948,1738,1635,1438,1365,1166,1092,568$ $\mathrm{cm}^{-1}$; HRMS (EI) m/z: [M+] Calcd for $\mathrm{C}_{20} \mathrm{H}_{17} \mathrm{NO}_{3} 319.1208$, found 319.1206; $\boldsymbol{R}_{f} 0.50$ (Hex/EtOAc, 7/3).

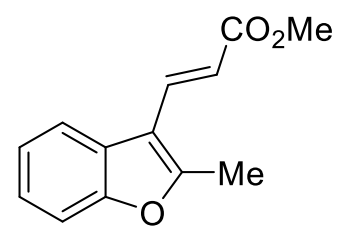

methyl (E)-3-(2-methylbenzofuran-3-yl)acrylate, $6:{ }^{S 3}$ pale yellow oil (64 mg, 98\%); ${ }^{1}$ H NMR (600 MHz, $\left.\mathbf{C D C l}_{3}\right) \delta 7.79(\mathrm{~d}, J=16.0 \mathrm{~Hz}, 1 \mathrm{H}), 7.75(\mathrm{dd}, J=6.0,3.0 \mathrm{~Hz}$, $1 \mathrm{H}), 7.46-7.41(\mathrm{~m}, 1 \mathrm{H}), 7.29(\mathrm{dd}, J=6.0,3.2 \mathrm{~Hz}, 2 \mathrm{H}), 6.50(\mathrm{~d}, J=16.0 \mathrm{~Hz}, 1 \mathrm{H})$, 3.83 (s, 3H), 2.57 (s, 3H); ${ }^{13}$ C NMR (151 MHz, CDCl 3 ) $\delta 168.1,159.0,154.5$, 135.5, 126.2, 124.5, 123.7, 120.5, 116.5, 112.8, 111.3, 51.8, 12.9; IR (neat): $v_{\max }=2925,1728,1648,1125$, $910,731 \mathrm{~cm}^{-1} ; \boldsymbol{R}_{f} 0.60(\mathrm{Hex} / \mathrm{EtOAc}, 4 / 1)$.

Ref S3. Ohno, S.; Qiu, J.; Miyazaki, R.; Aoyama, H.; Murai, K.; Hasegawa, J.-y.; Arisawa, M., Ni-Catalyzed Cycloisomerization between 3-Phenoxy Acrylic Acid Derivatives and Alkynes via Intramolecular Cleavage and Formation of the C-O Bond To Give 2,3-Disubstituted Benzofurans. Org. Lett. 2019, 21, 8400-8403. 


\section{Analytical data for carbazole (8):}

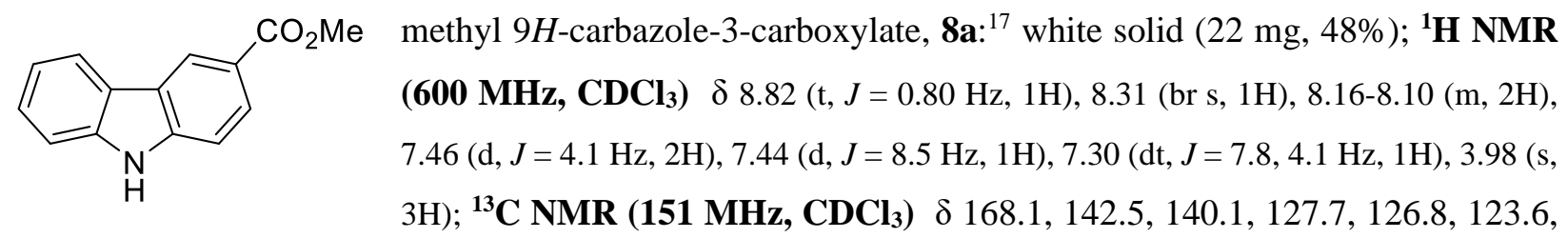

123.4, 123.1, 121.7, 120.9, 120.6, 111.1, 110.3, 52.2; IR (neat): $v_{\max }=2948,1738,1604,1434,1365$, $1229,1166,1093,541 \mathrm{~cm}^{-1} ; \boldsymbol{R}_{f} 0.56(\mathrm{Hex} / \mathrm{EtOAc}, 4 / 1)$.

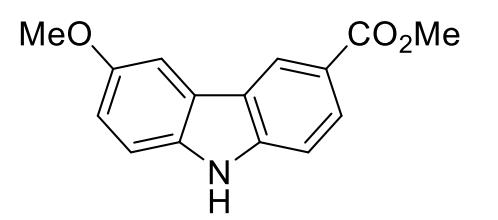

methyl 6-methoxy-9H-carbazole-3-carboxylate; $\mathbf{8 b}:{ }^{17}$ white solid (33 mg, 64\%); ${ }^{1} \mathbf{H}$ NMR (600 MHz, $\left.\mathbf{c d c l}_{3}\right) \delta 8.78(\mathrm{~d}, J=1.6 \mathrm{~Hz}, 1 \mathrm{H}), 8.31$ (br s, $1 \mathrm{H}), 8.11(\mathrm{dd}, J=8.5,1.6 \mathrm{~Hz}, 1 \mathrm{H}), 7.59(\mathrm{~d}, J=2.5 \mathrm{~Hz}, 1 \mathrm{H}), 7.40(\mathrm{~d}, J=$ $8.6 \mathrm{~Hz}, 1 \mathrm{H}), 7.35$ (d, $J=8.6 \mathrm{~Hz}, 1 \mathrm{H}), 7.10(\mathrm{dd}, J=8.8,2.5 \mathrm{~Hz}, 1 \mathrm{H}), 3.98$ (s, 3H), 3.93 (s, 3H). ${ }^{\mathbf{1 3}} \mathbf{C}$ NMR (151 MHz, cdcl $\left._{3}\right) \delta 169.5,154.7,142.3,134.9,127.0,124.1,123.3,122.8$, 120.4, 116.2, 111.9, 110.5, 103.9, 56.7, 52.2. IR (neat): $v_{\max }=2948,1738,1628,1456,1434,1306,1165$, $1097,579 \mathrm{~cm}^{-1} ; \boldsymbol{R}_{f} 0.52(\mathrm{Hex} / \mathrm{EtOAc}, 4 / 1)$.

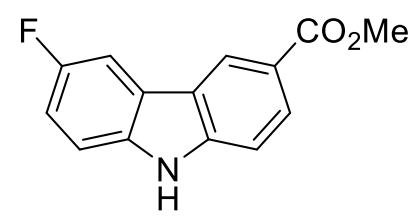

methyl 6-fluoro-9H-carbazole-3-carboxylate; $8 \mathbf{c}^{.17}$ white solid (36 mg, 75\%); ${ }^{1}$ H NMR (600 MHz, $\left.\mathbf{C D C l}_{3}\right) \delta 8.76(\mathrm{~s}, 1 \mathrm{H}), 8.32$ (br s, 1H), 8.15 (dd, $J=8.7$, $2.0 \mathrm{~Hz}, 1 \mathrm{H}), 7.77(\mathrm{dd}, J=8.7,2.0 \mathrm{~Hz}, 1 \mathrm{H}), 7.43(\mathrm{~d}, J=8.5 \mathrm{~Hz}, 1 \mathrm{H}), 7.38(\mathrm{dd}$, $J=8.7,4.2 \mathrm{~Hz}, 1 \mathrm{H}), 7.20(\mathrm{td}, J=8.9,2.6 \mathrm{~Hz}, 1 \mathrm{H}), 3.98(\mathrm{~s}, 2 \mathrm{H}) ;{ }^{13} \mathbf{C} \mathbf{N M R}$

(151 MHz, $\left.\mathbf{C D C l}_{3}\right) \delta 167.9,158.9,157.4,143.4,136.4,128.2,124.3,124.2,123.4,123.1,123.1,121.7$, 114.8, 114.6, 111.8, 111.7, 110.6, 110.1, 106.7, 106.6, 52.2; IR (neat): $v_{\max }=2949,1738,1612,1496$, $1434,1365,1161,1092,770 \mathrm{~cm}^{-1} ; \boldsymbol{R}_{f} 0.52$ (Hex/EtOAc, 4/1).

Ref S4. Rasheed, S.; Rao, D. N.; Reddy, K. R.; Aravinda, S.; Vishwakarma, R. A.; Das, P., C-N bond formation via $\mathrm{Cu}$-catalyzed cross-coupling with boronic acids leading to methyl carbazole-3-carboxylate: synthesis of carbazole alkaloids. RSC Adv. 2014, 4, 4960-4969. 

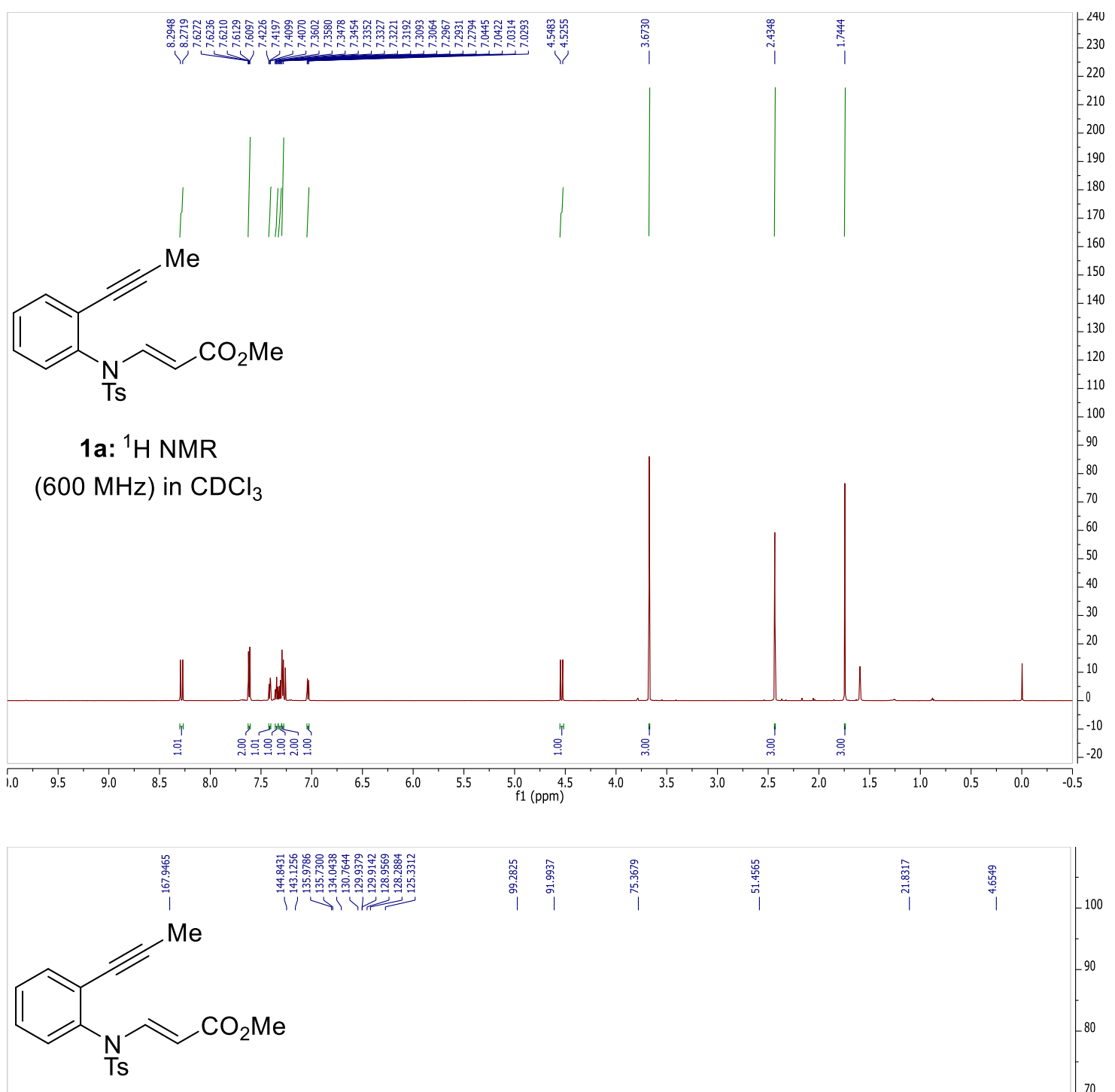

1a: ${ }^{13} \mathrm{C}$ NMR

$(151 \mathrm{MHz})$ in $\mathrm{CDCl}_{3}$

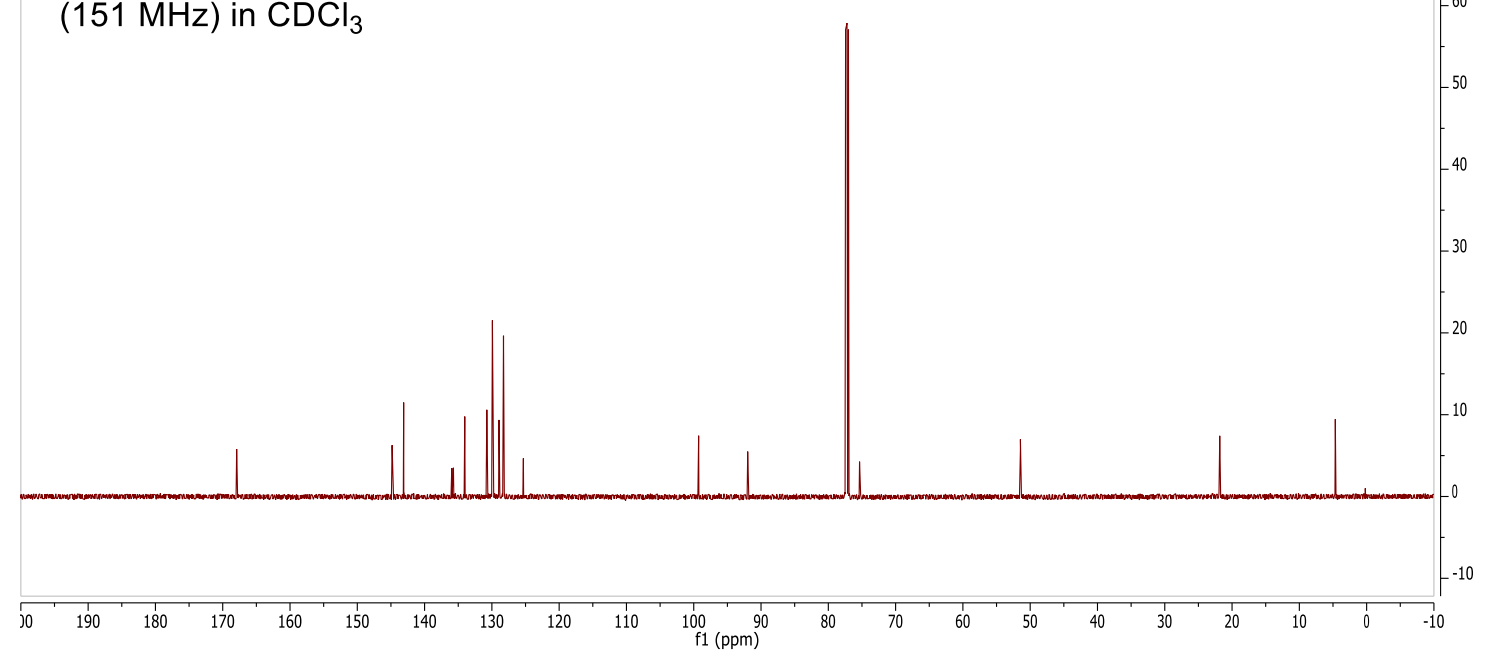



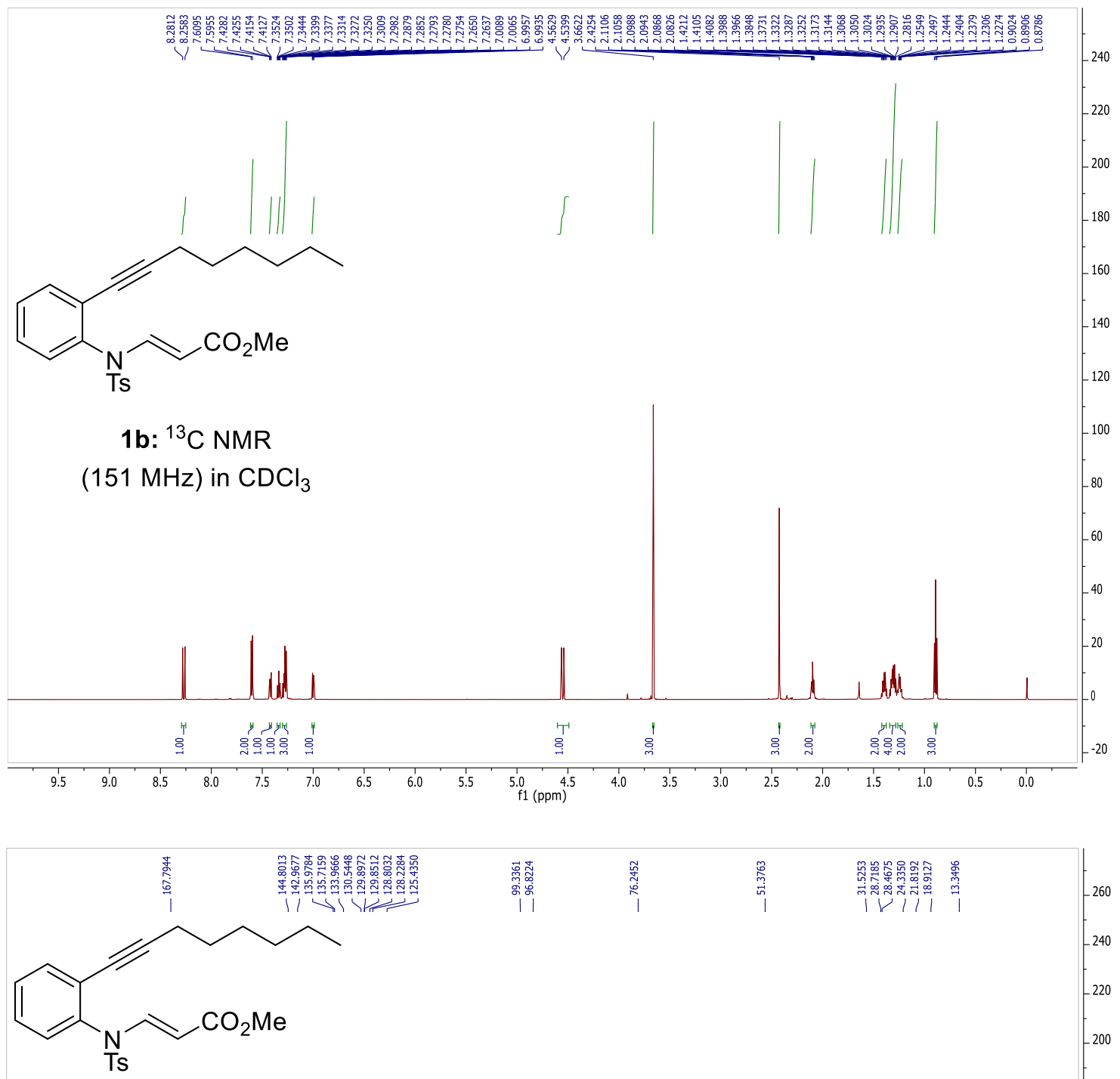

1b: ${ }^{13} \mathrm{C}$ NMR

$(151 \mathrm{MHz})$ in $\mathrm{CDCl}_{3}$

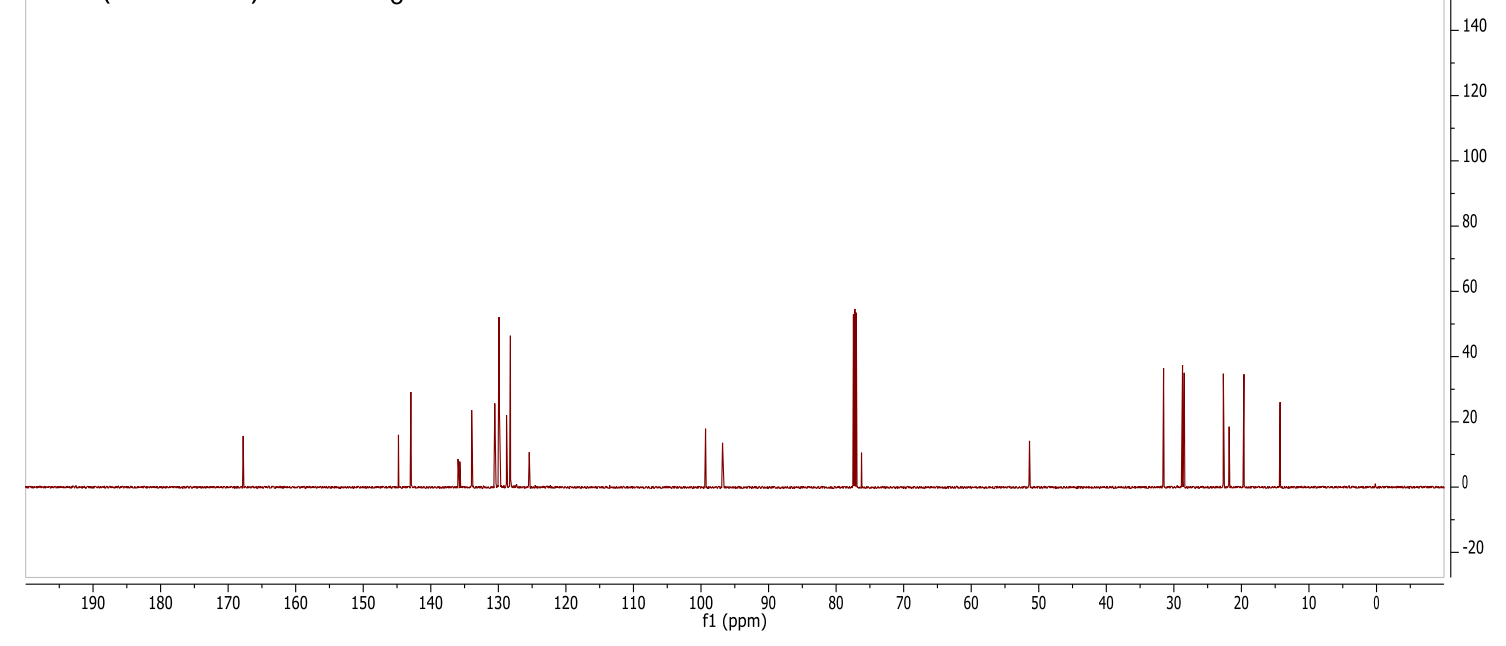



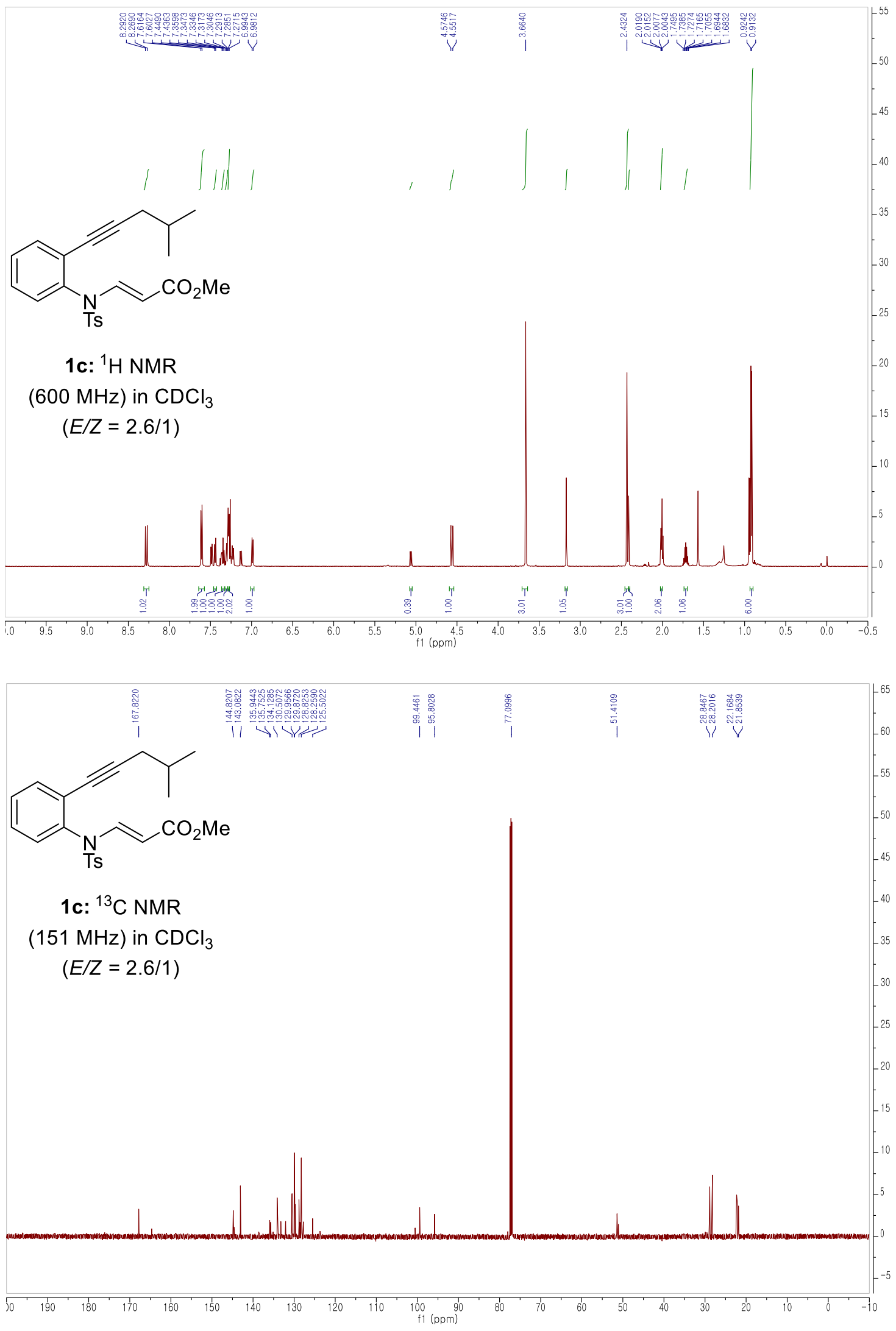


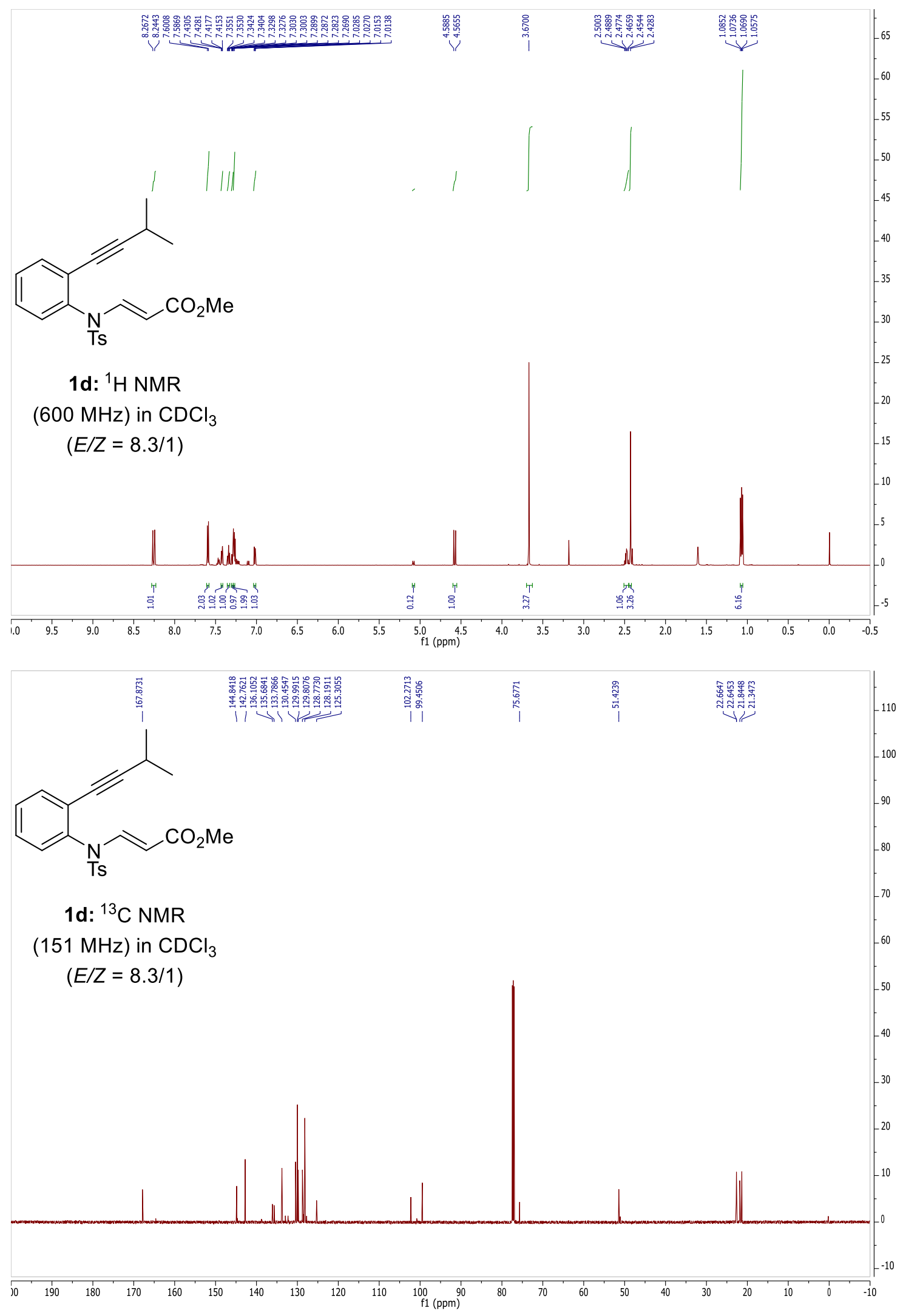



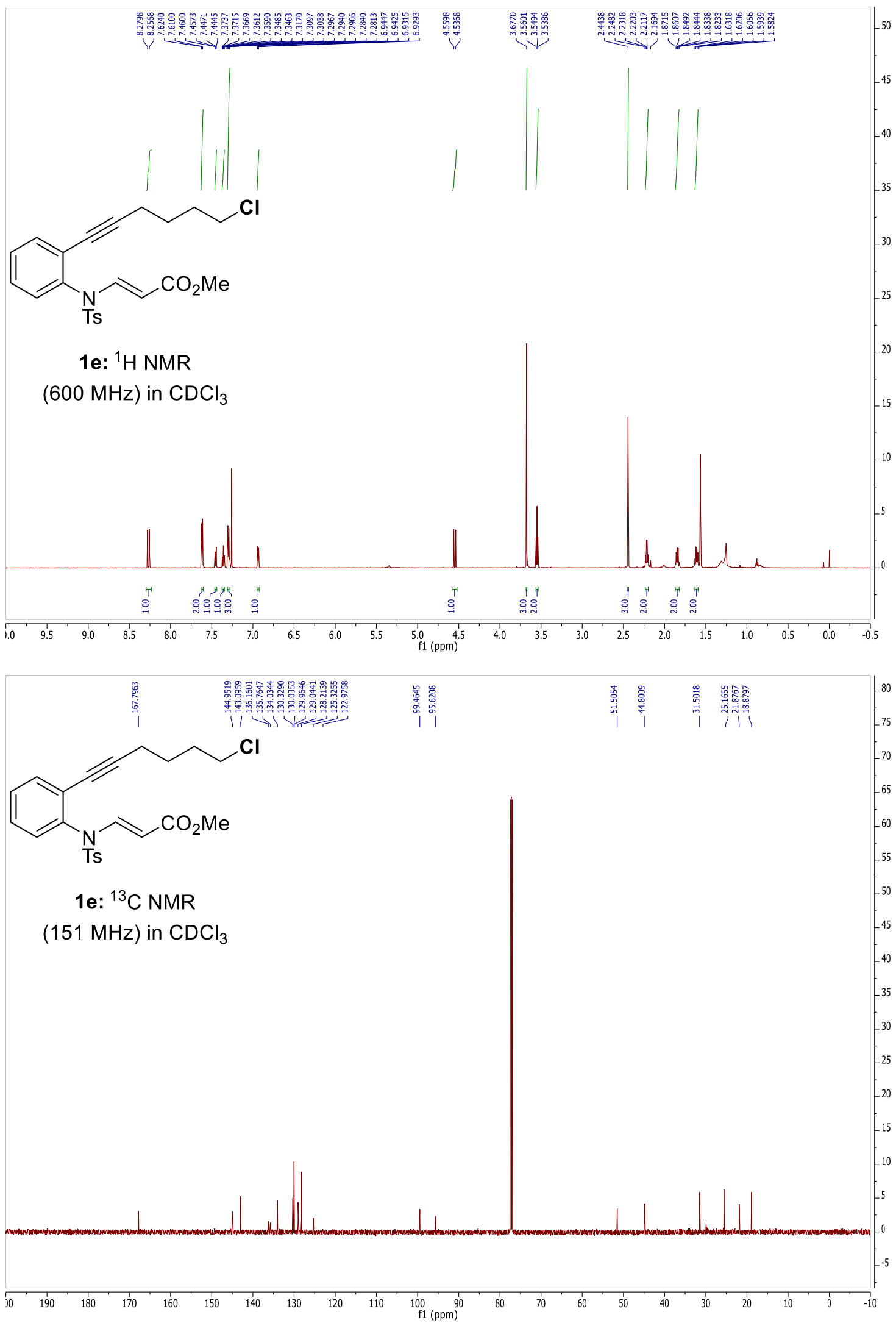


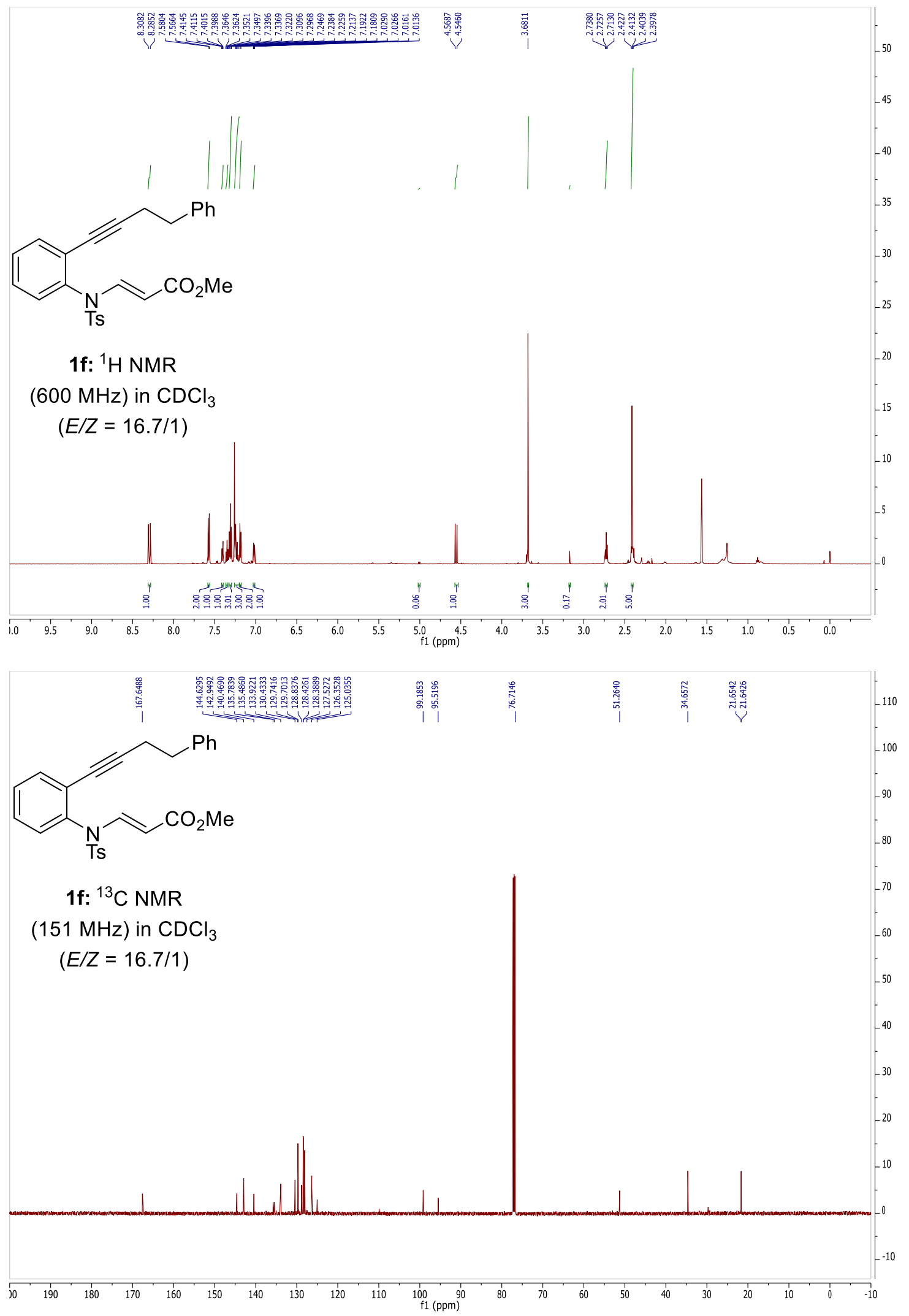



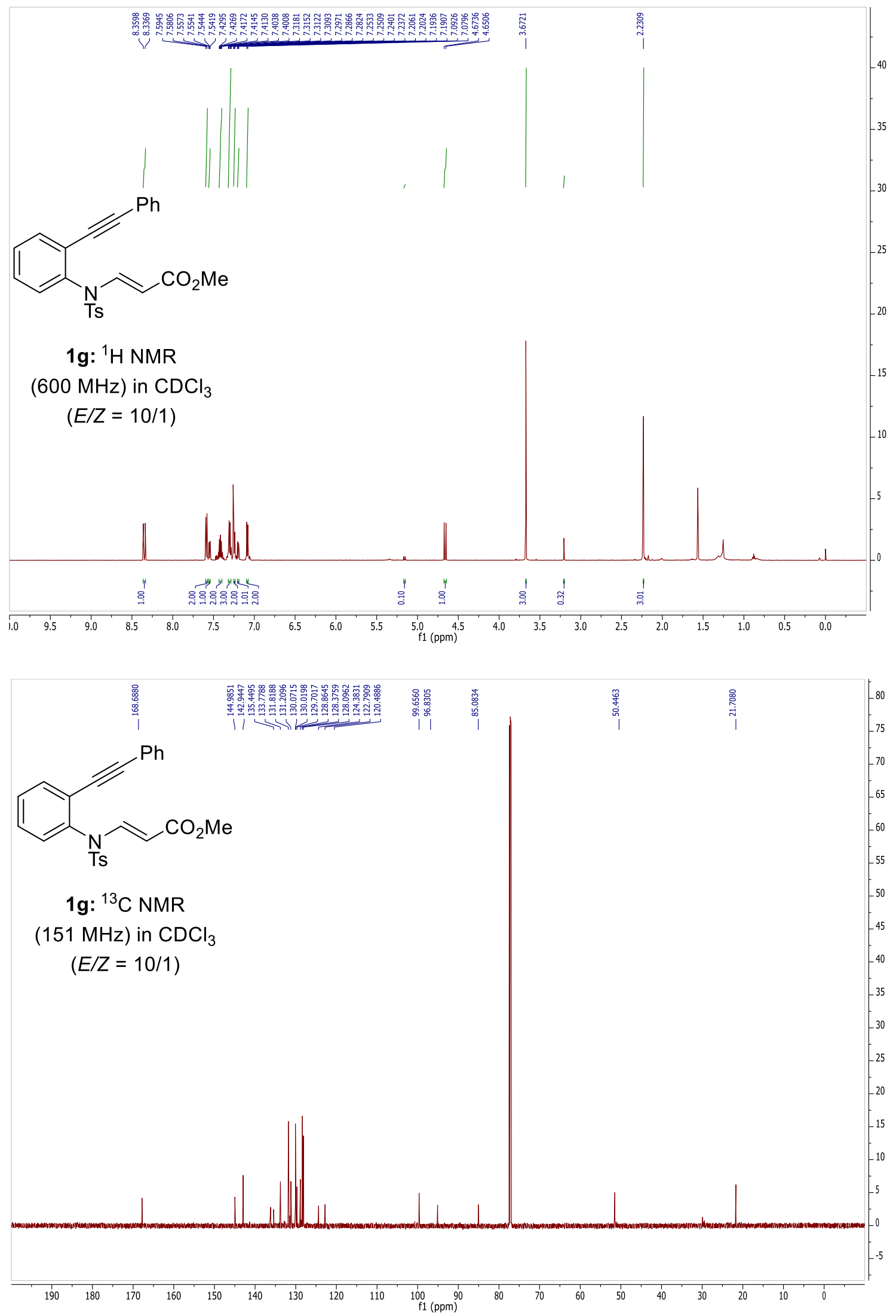

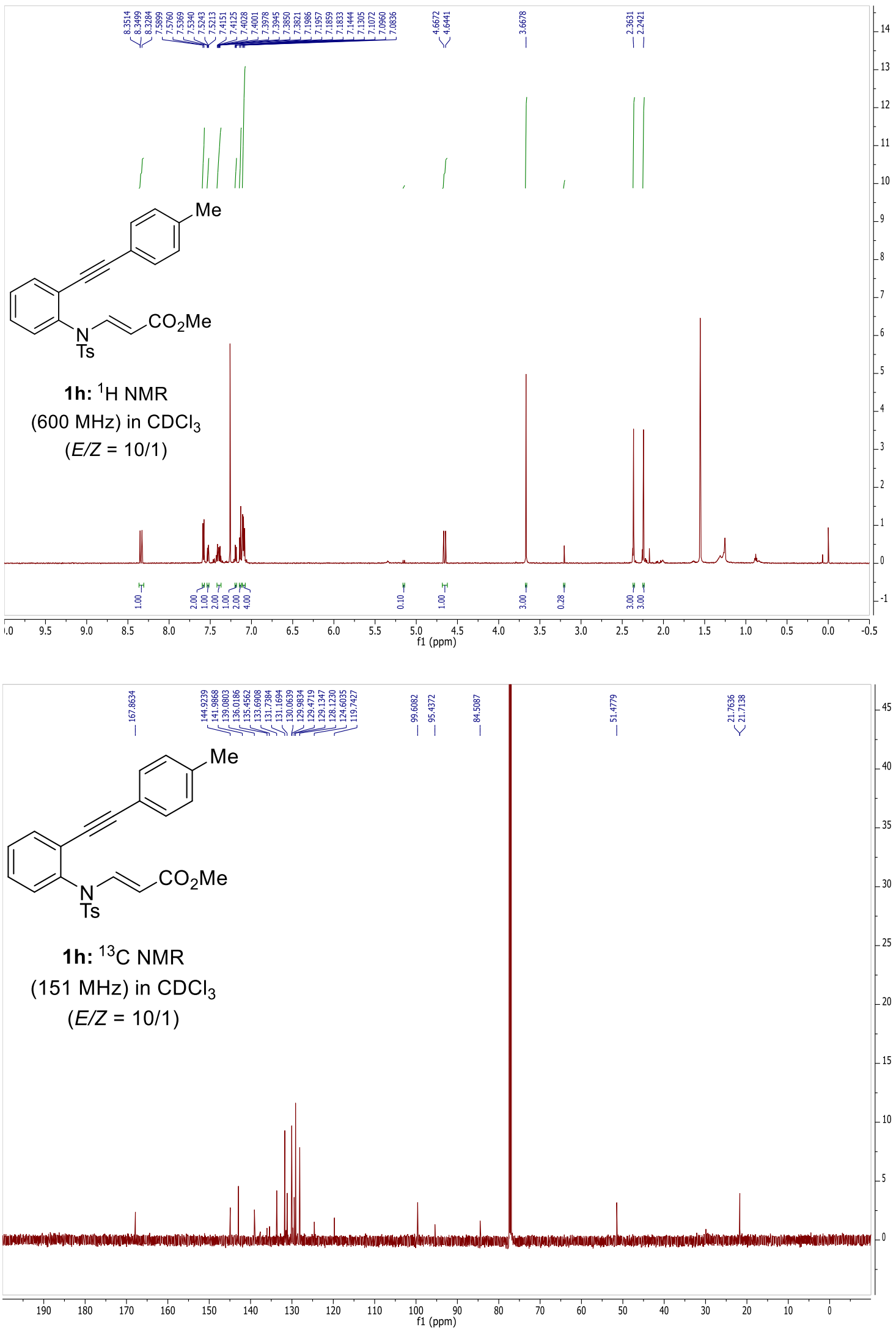

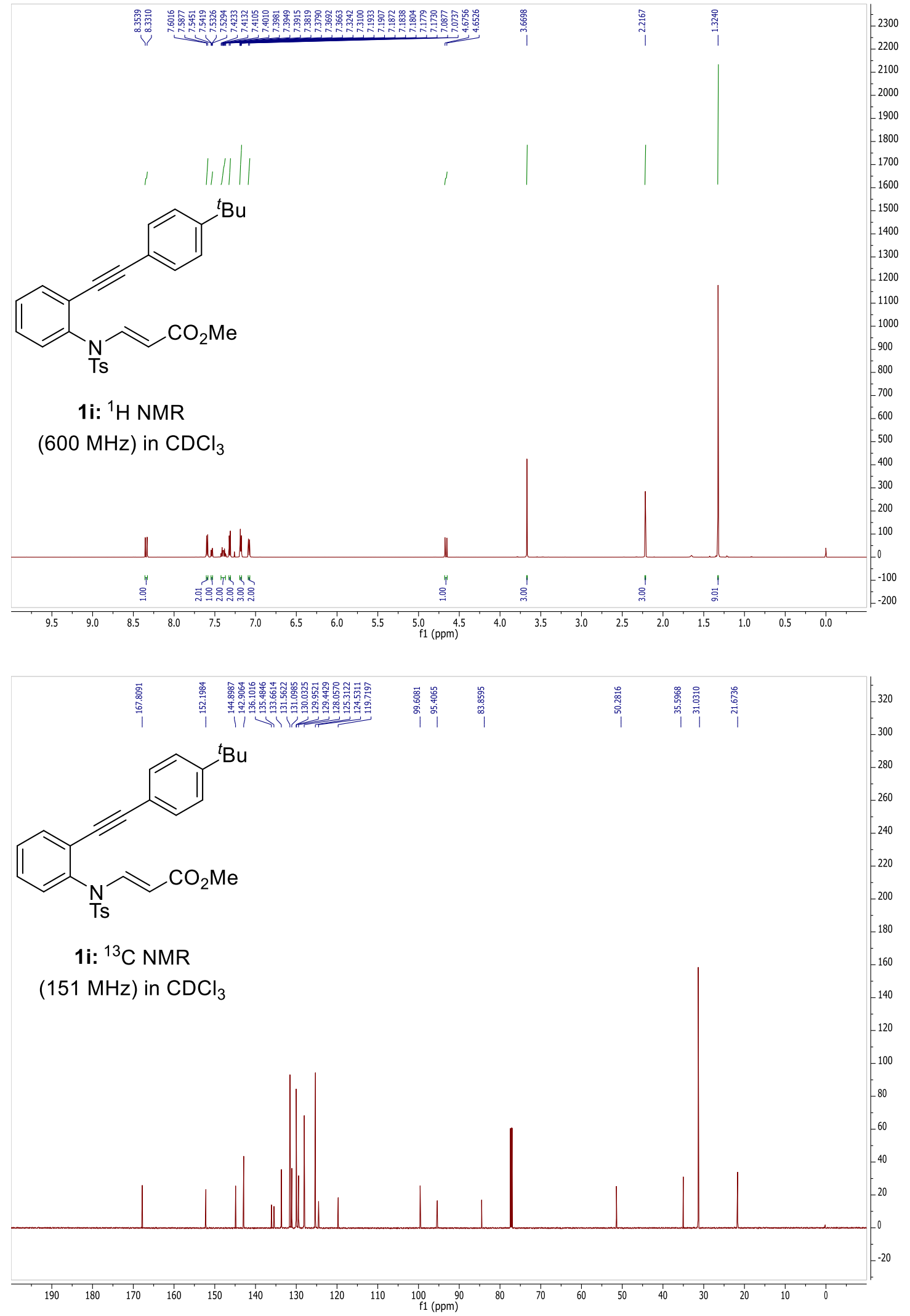

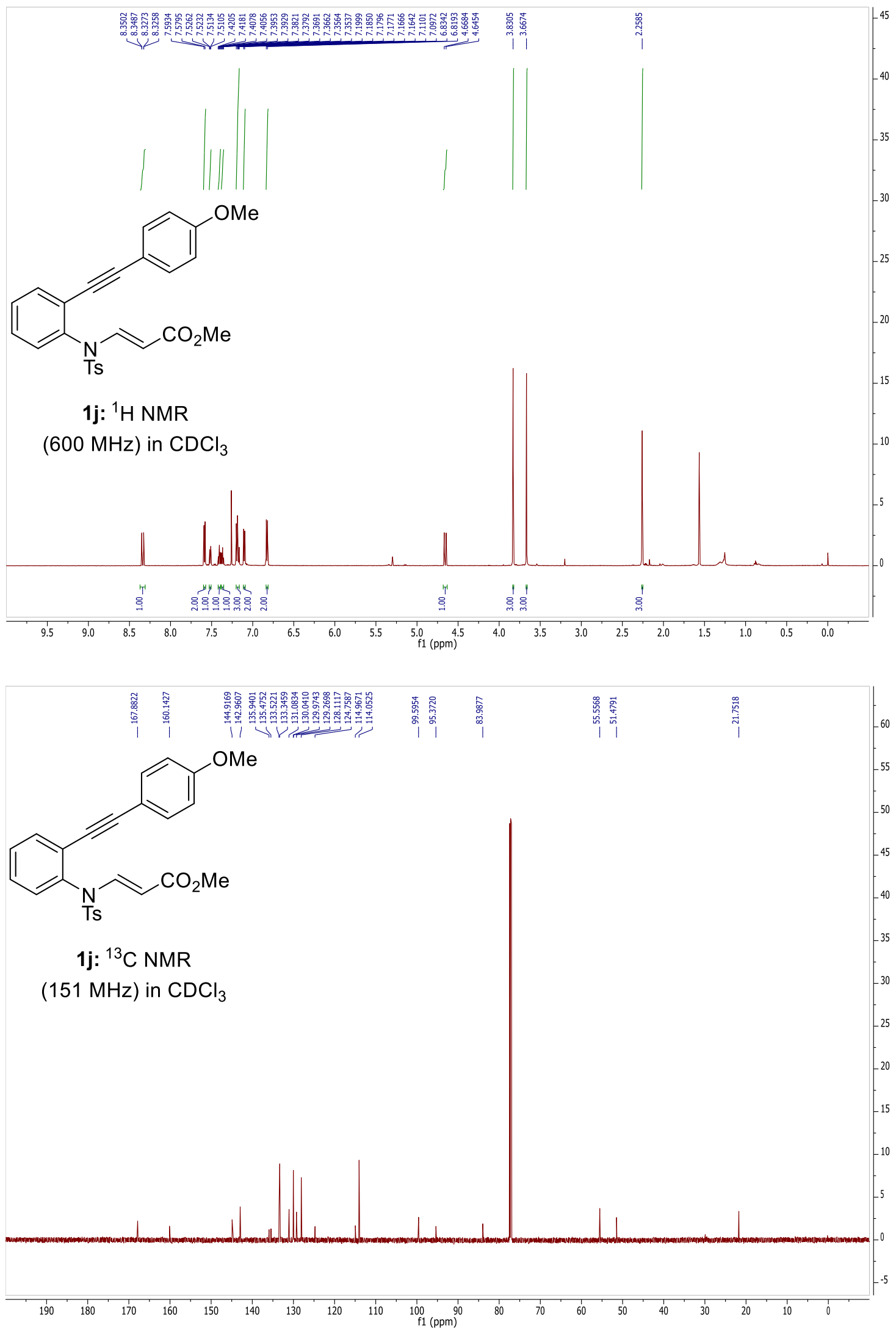

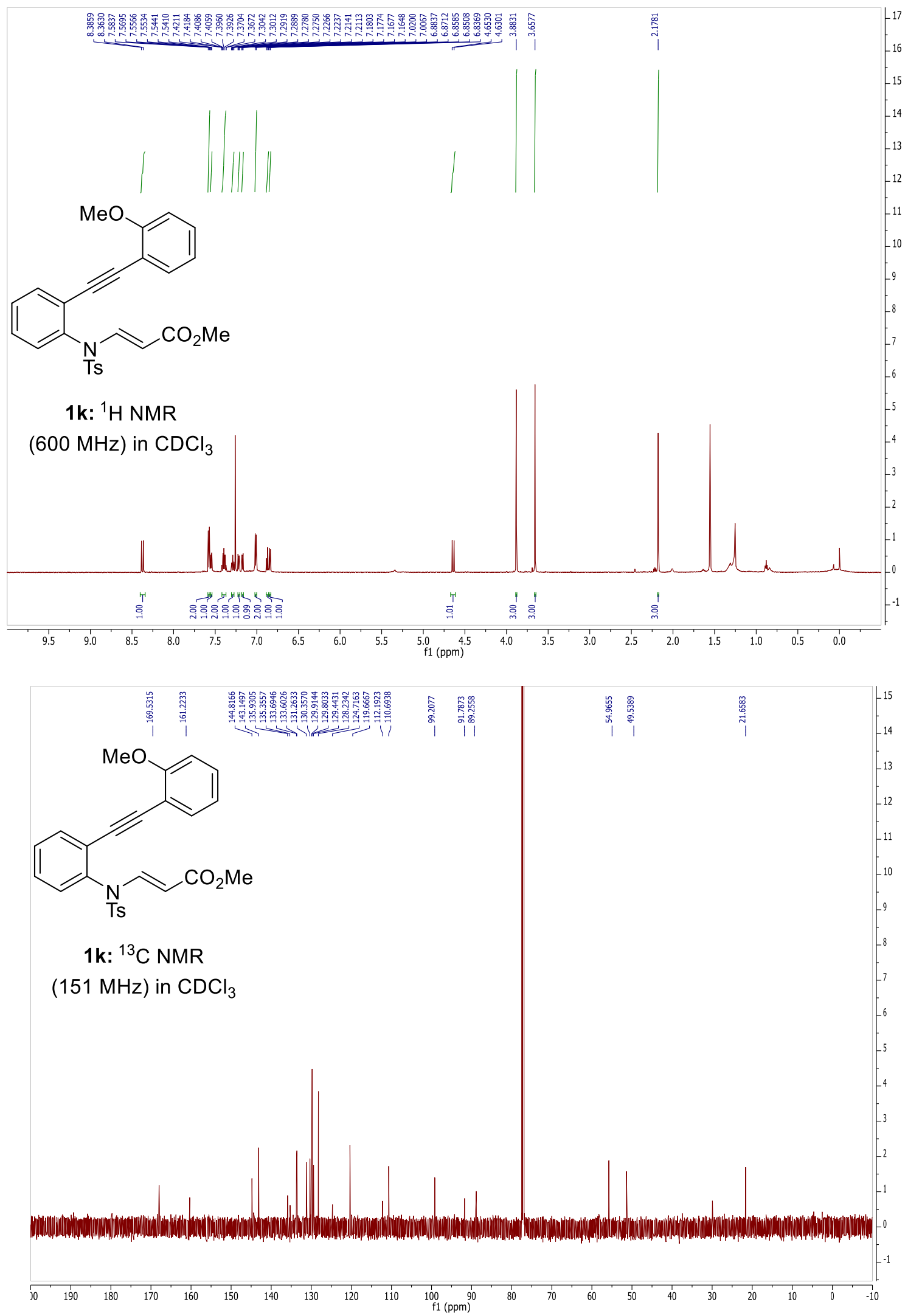

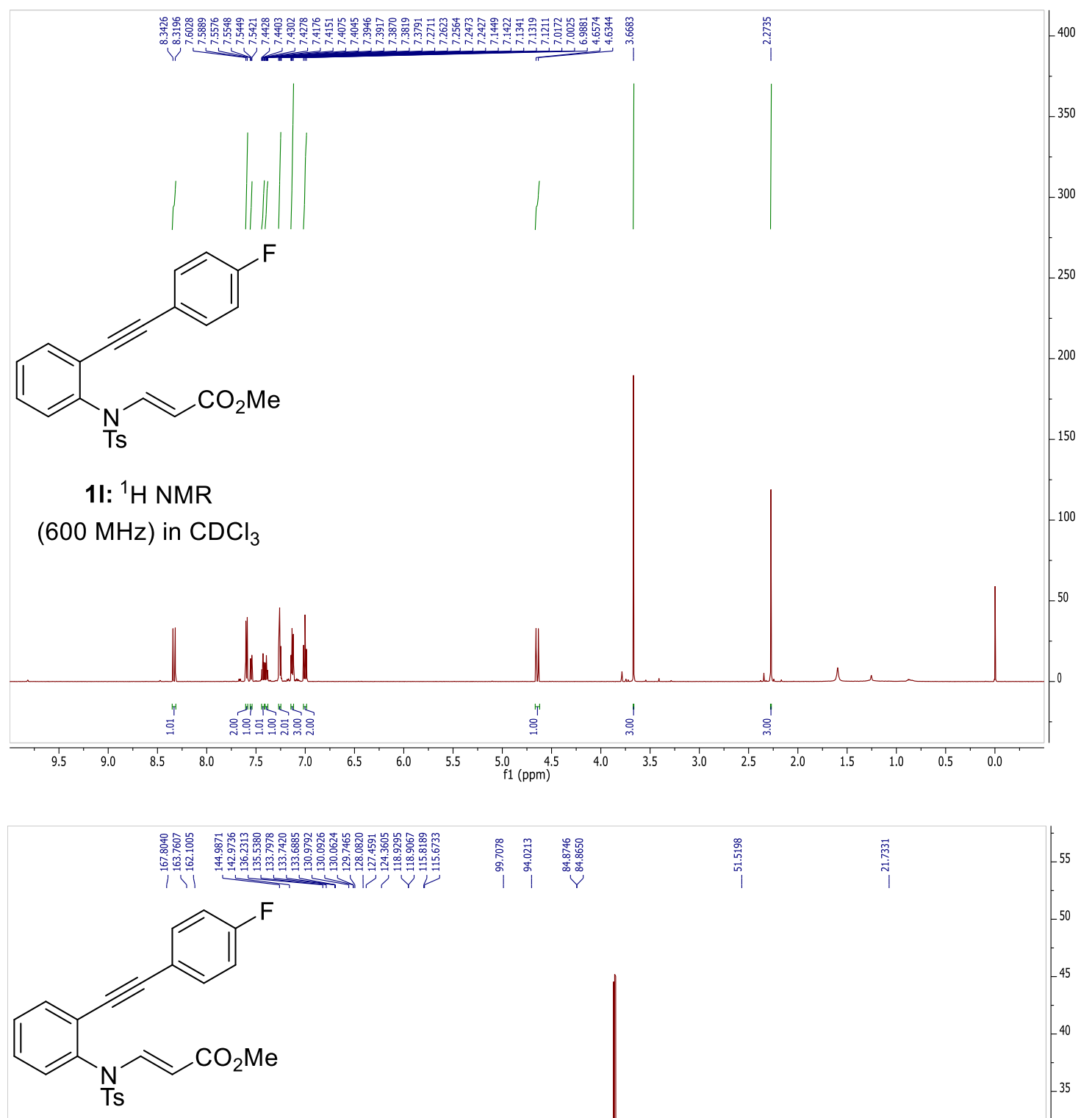

1I: ${ }^{13} \mathrm{C}$ NMR (151 MHz) in $\mathrm{CDCl}_{3}$

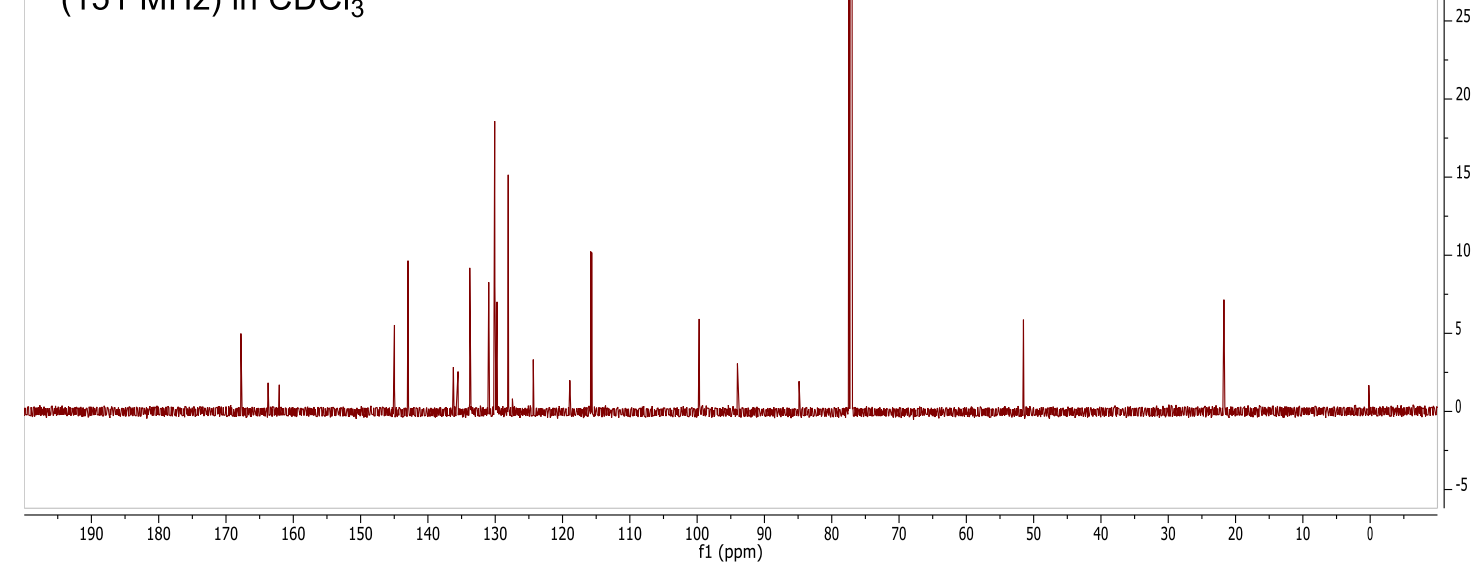



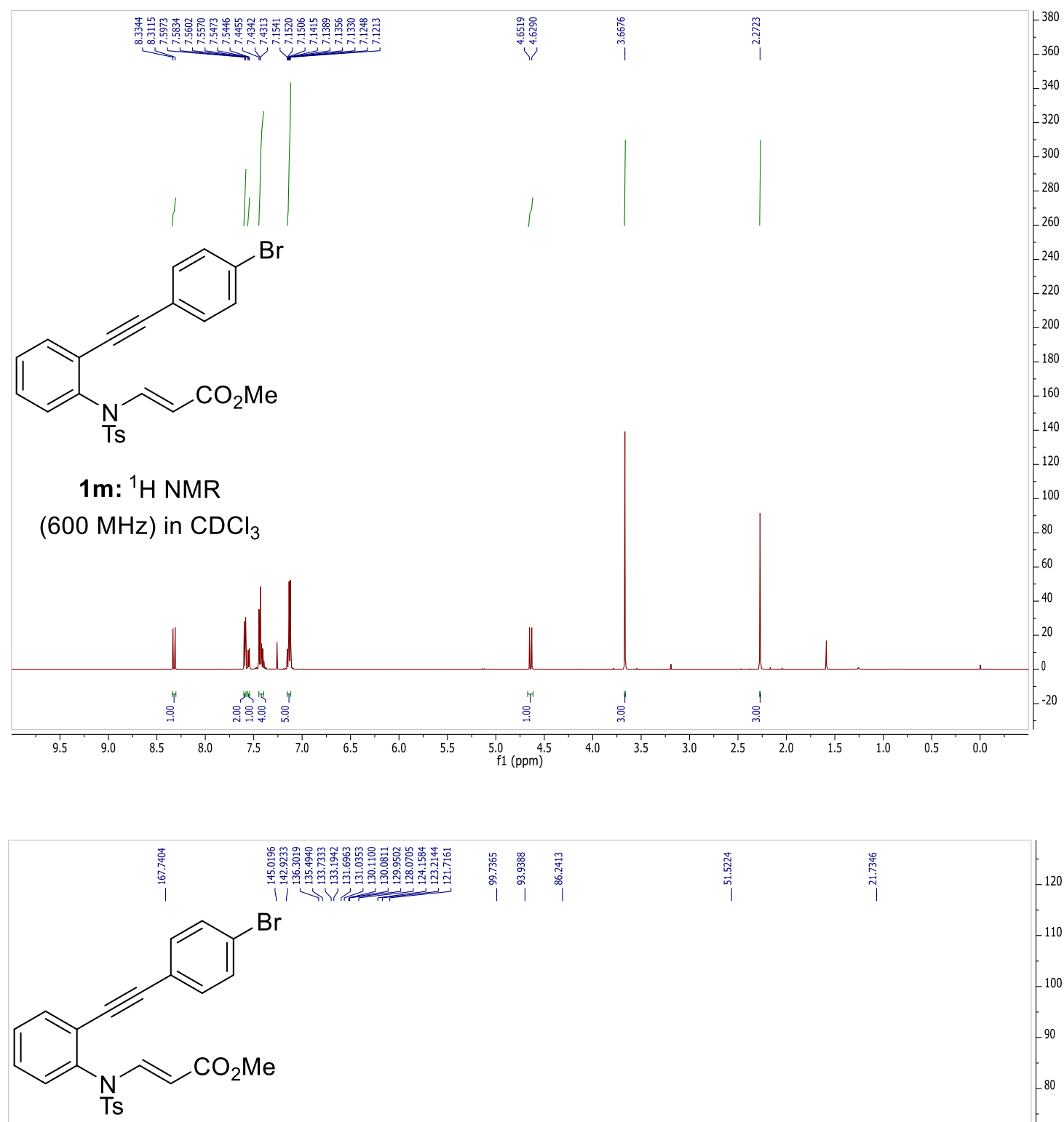

$1 \mathrm{~m}:{ }^{13} \mathrm{C}$ NMR (151 MHz) in $\mathrm{CDCl}_{3}$

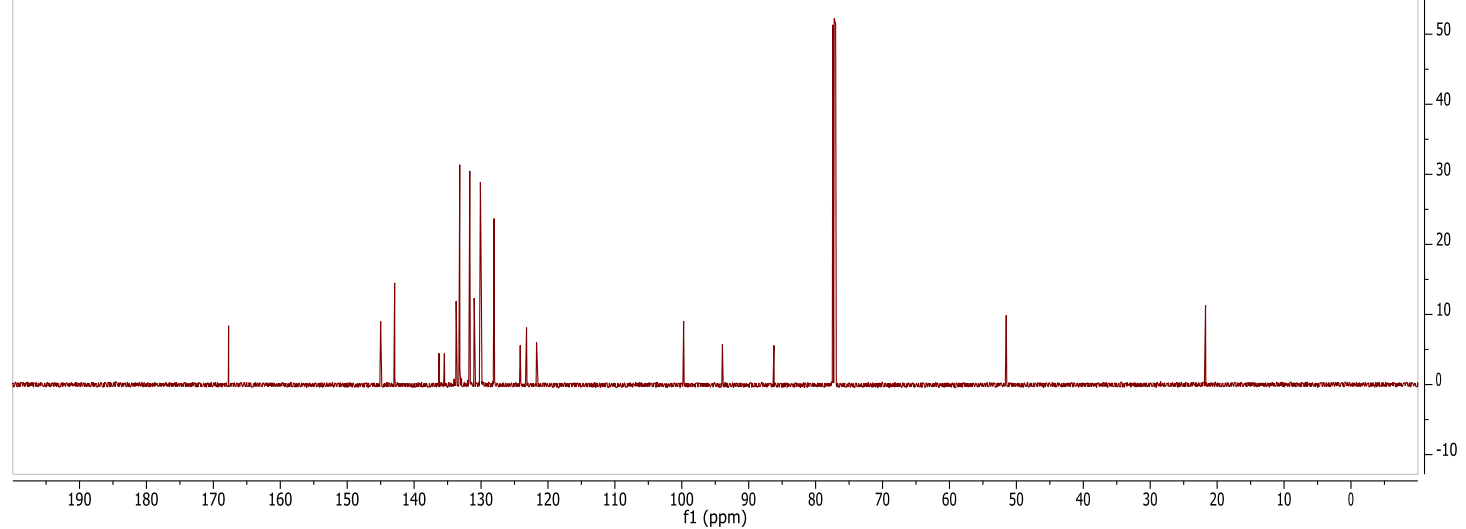



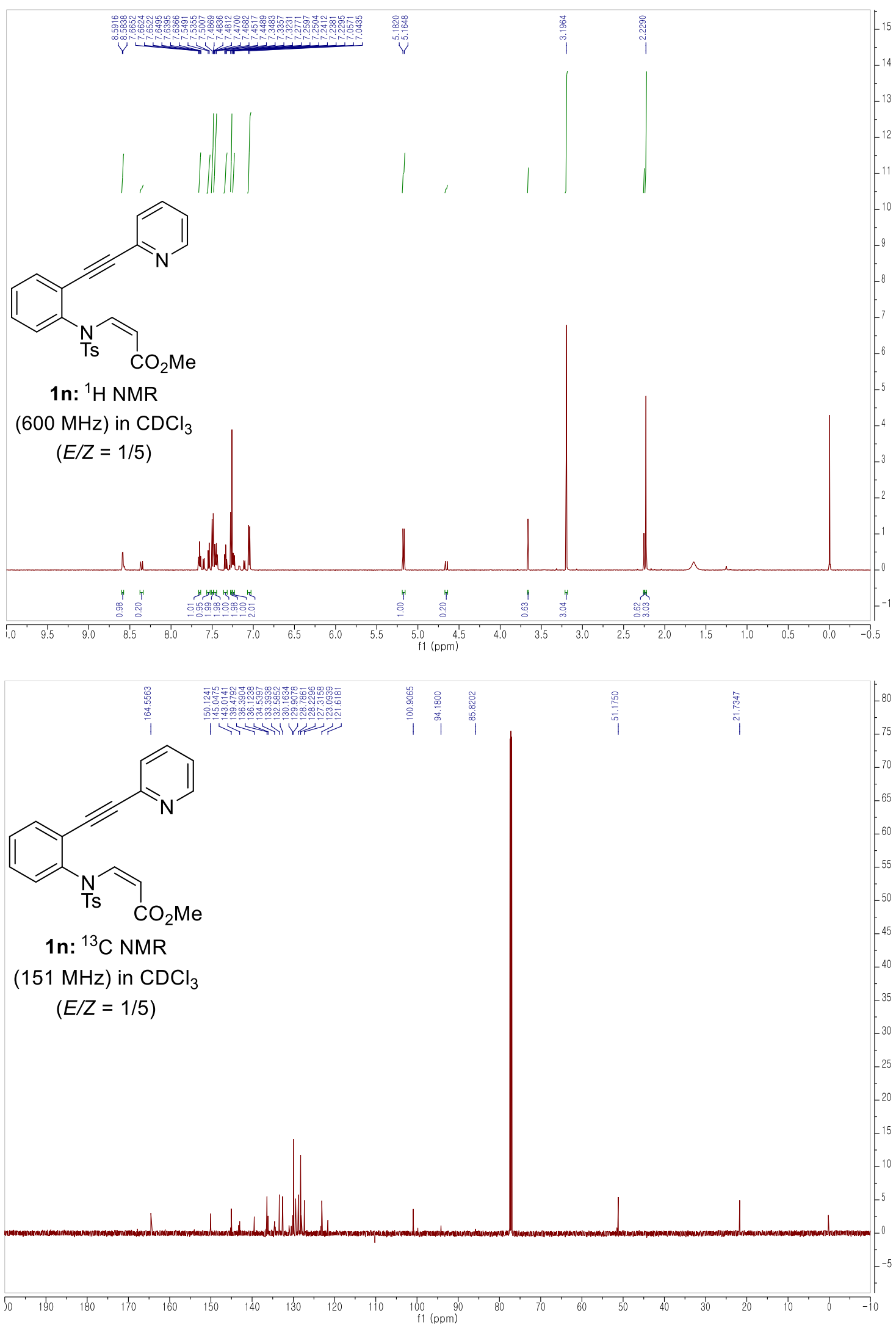

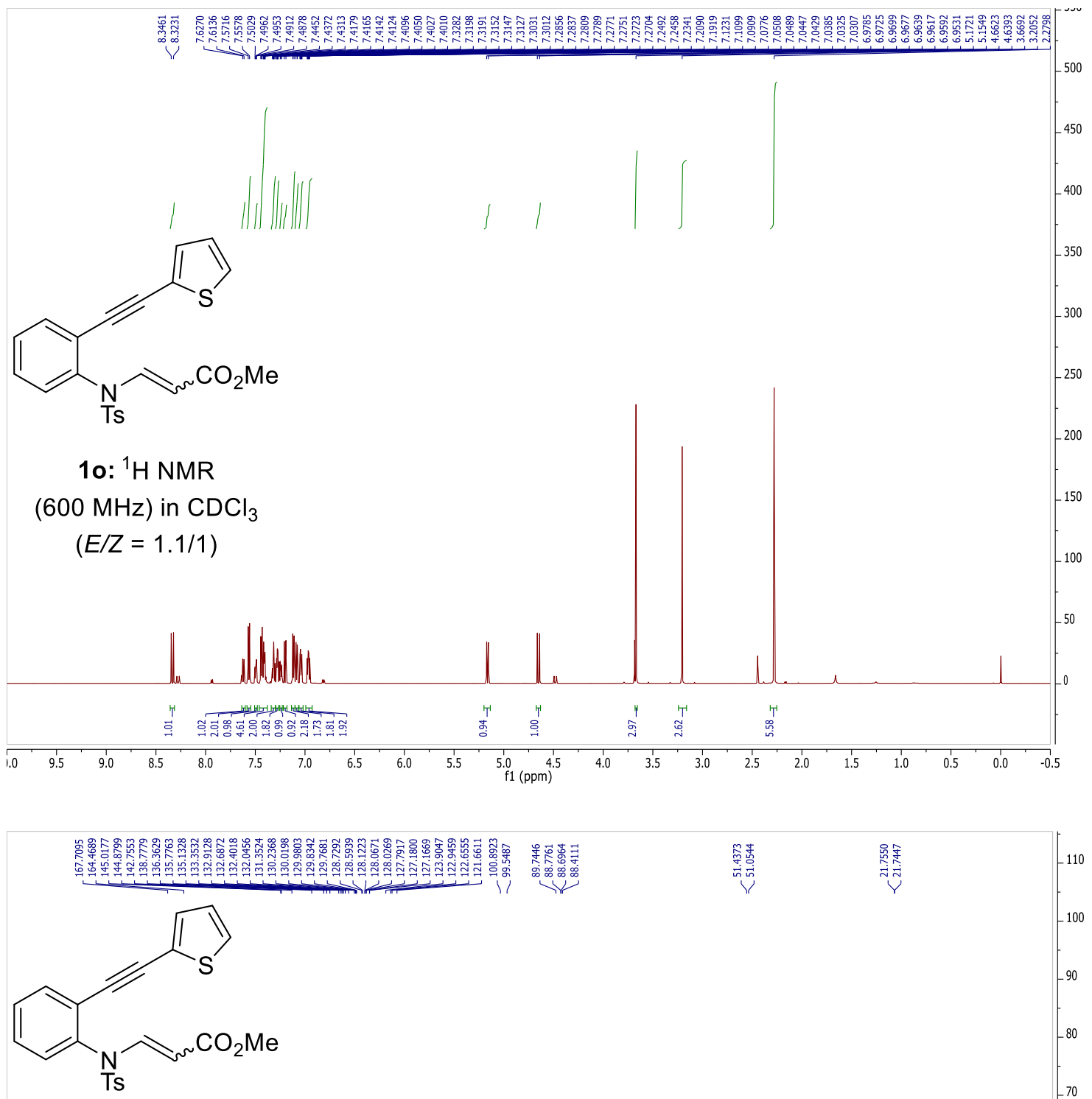

10: ${ }^{13} \mathrm{C}$ NMR

(151 MHz) in $\mathrm{CDCl}_{3}$

$(E / Z=1.1 / 1)$

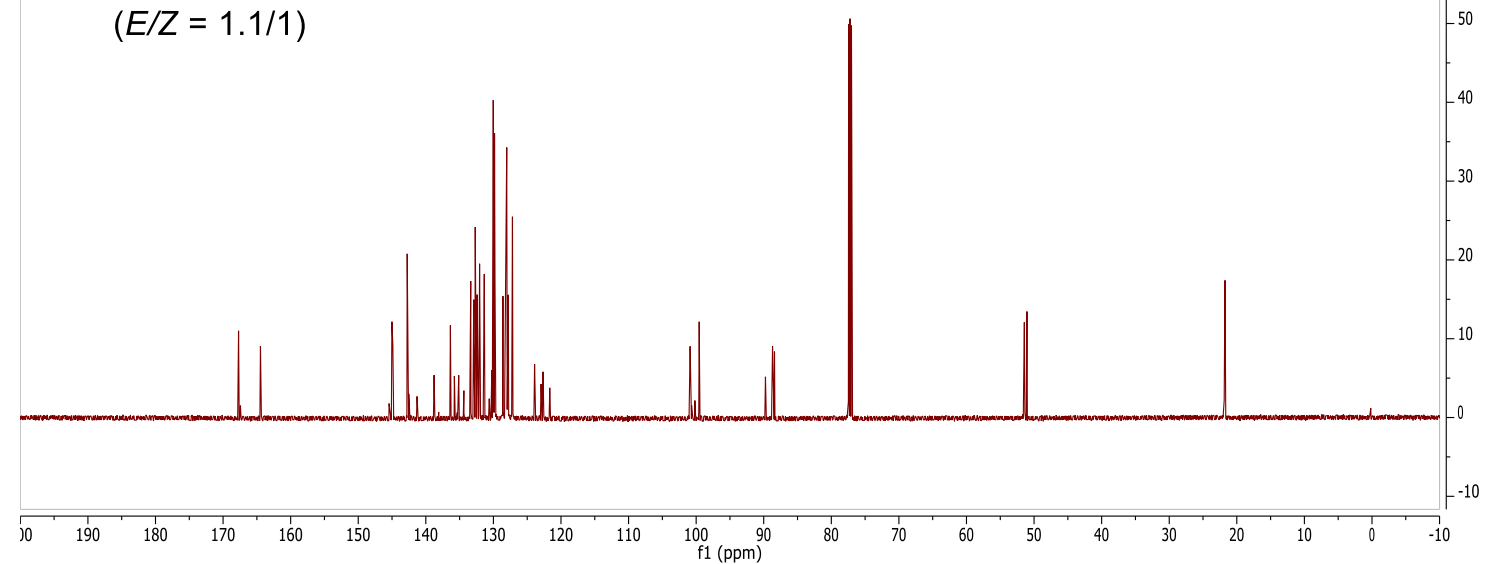



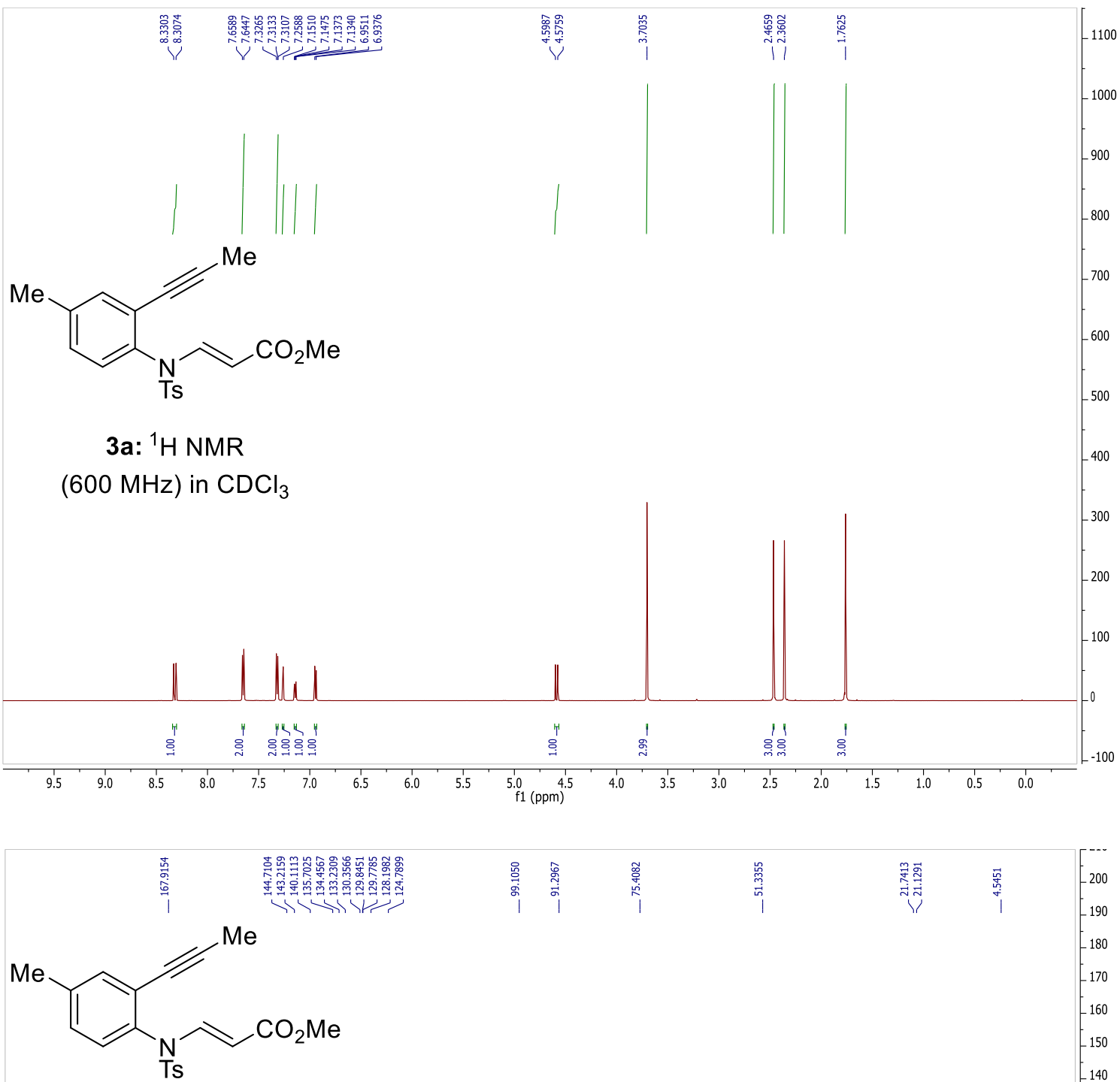

3a: ${ }^{13} \mathrm{C}$ NMR

$(151 \mathrm{MHz})$ in $\mathrm{CDCl}_{3}$

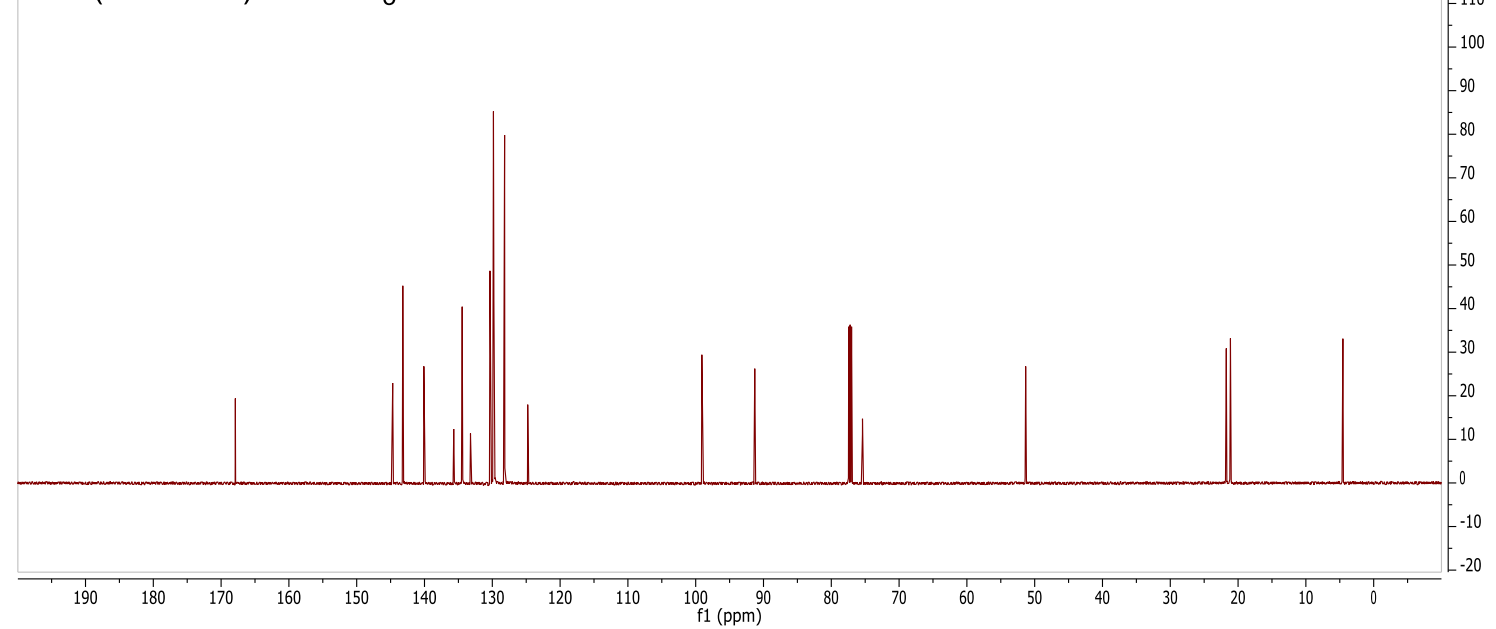



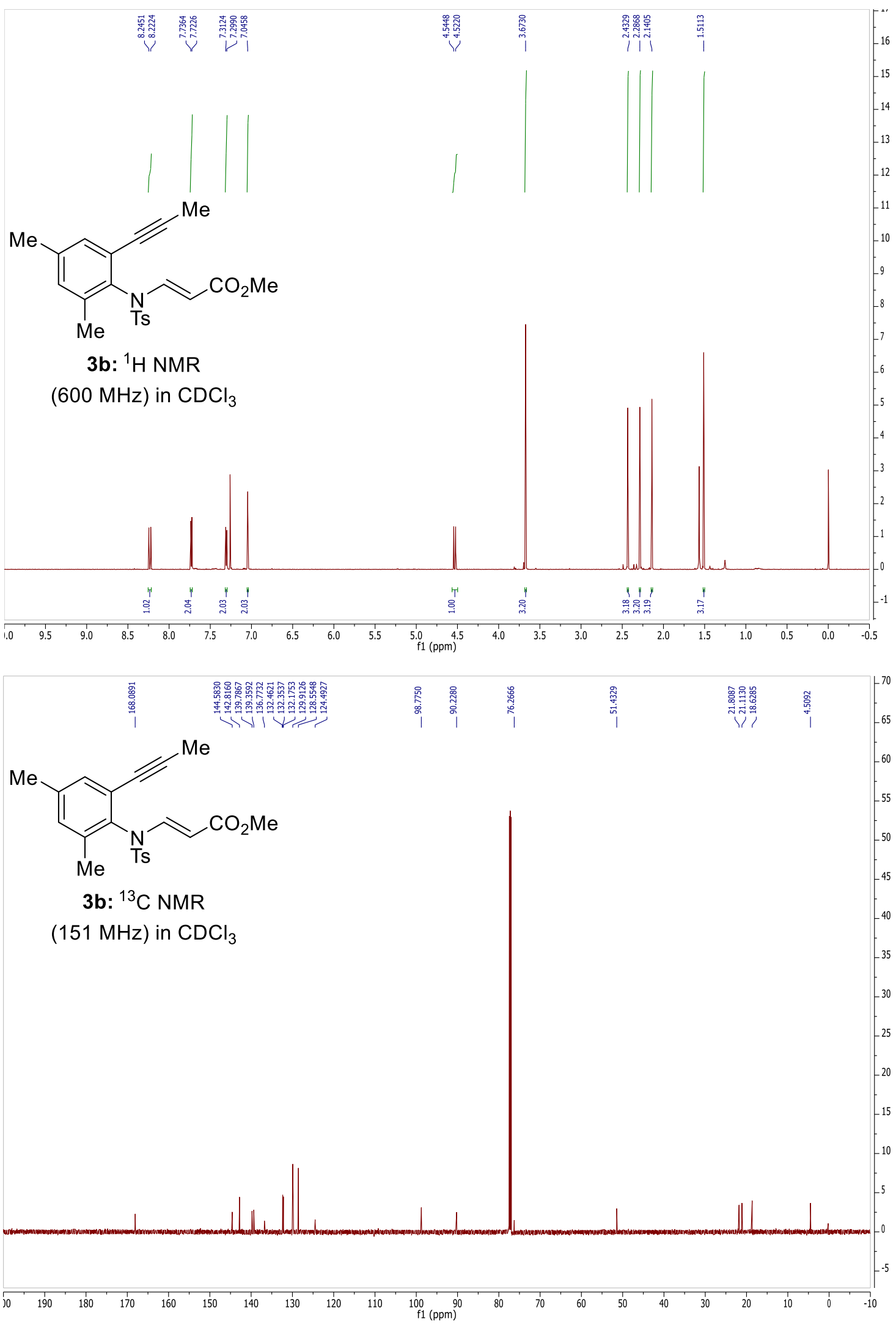

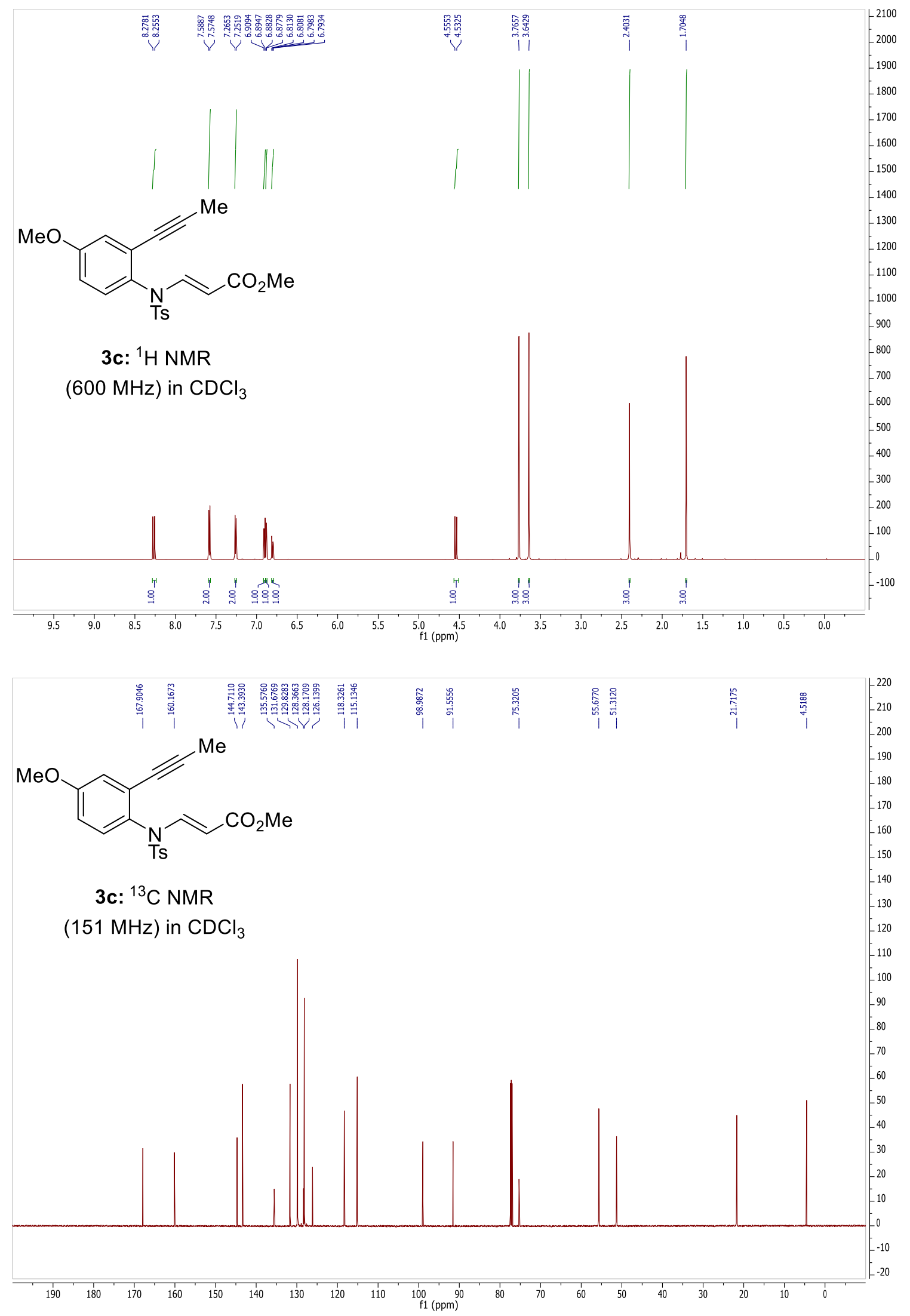

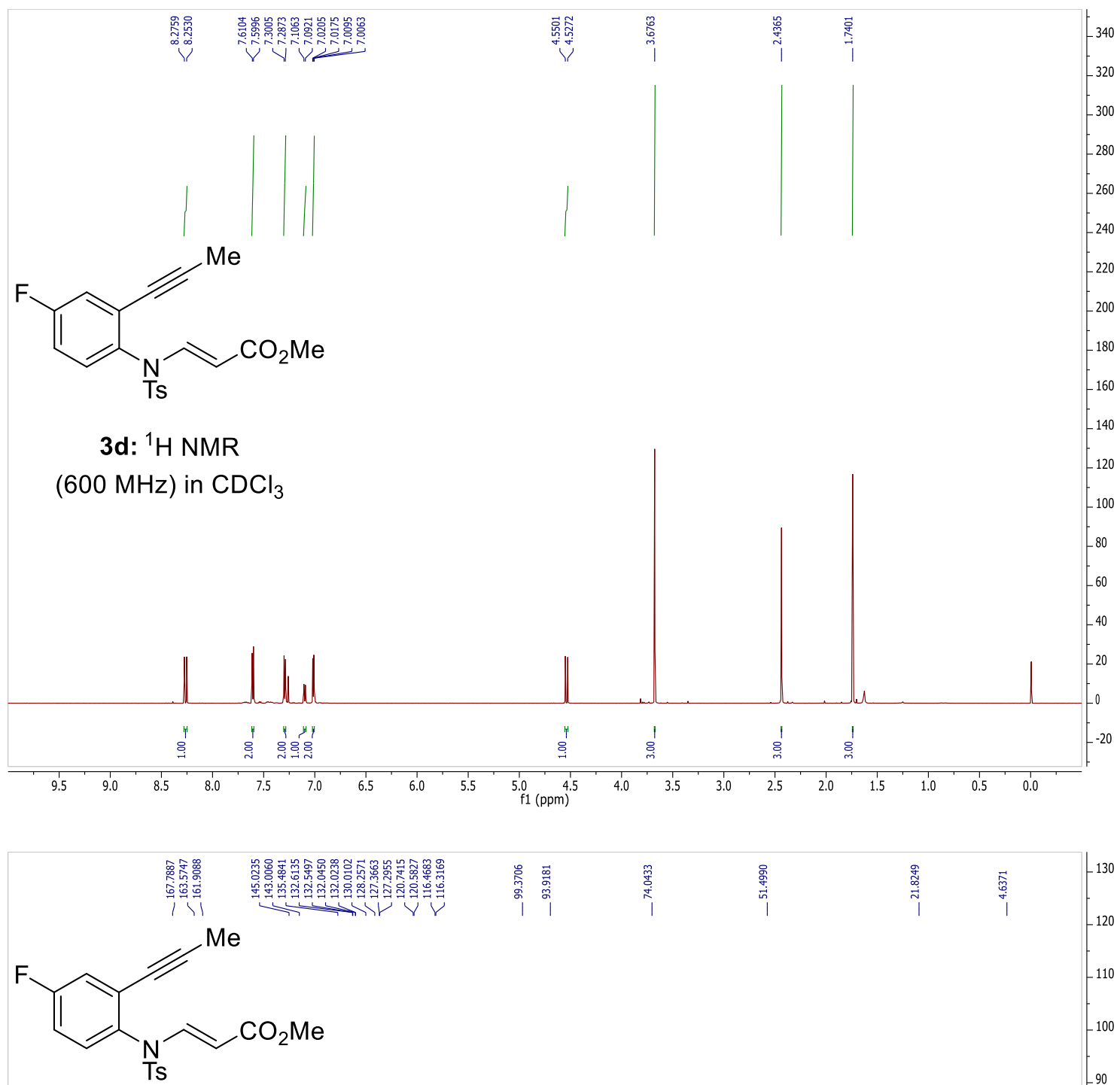

3d: ${ }^{13} \mathrm{C}$ NMR

$(151 \mathrm{MHz})$ in $\mathrm{CDCl}_{3}$

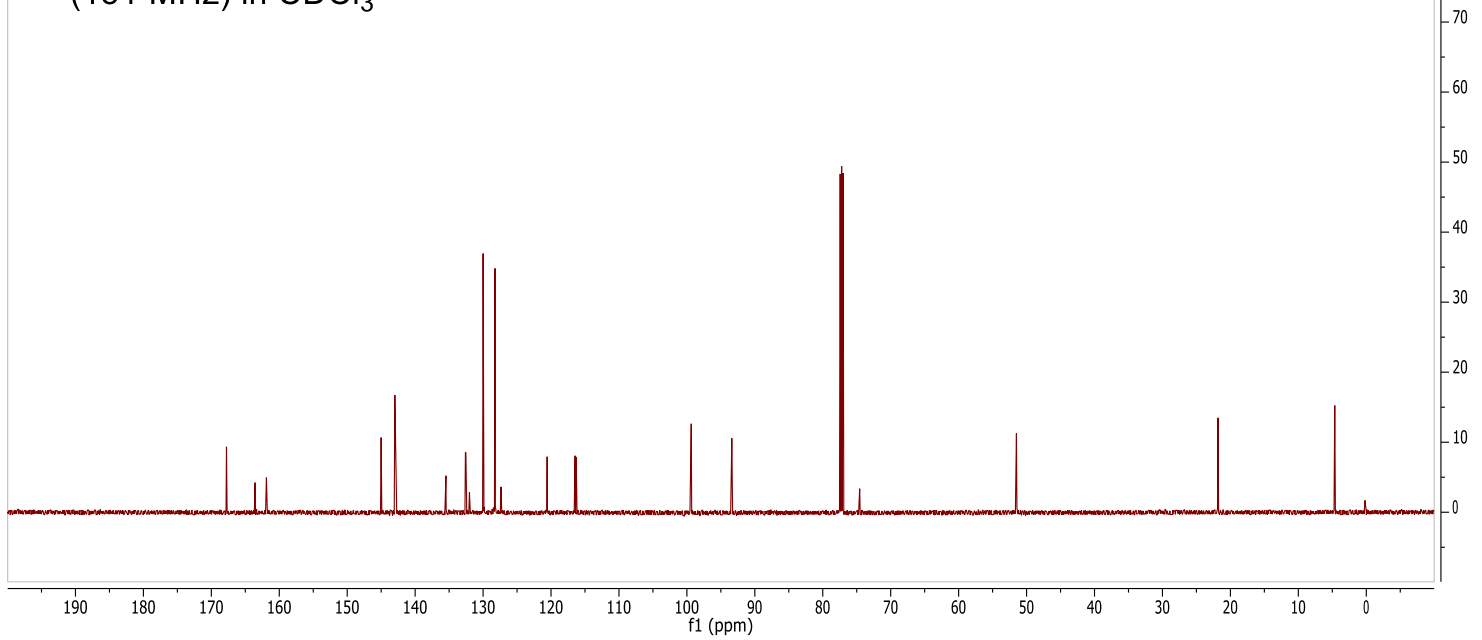



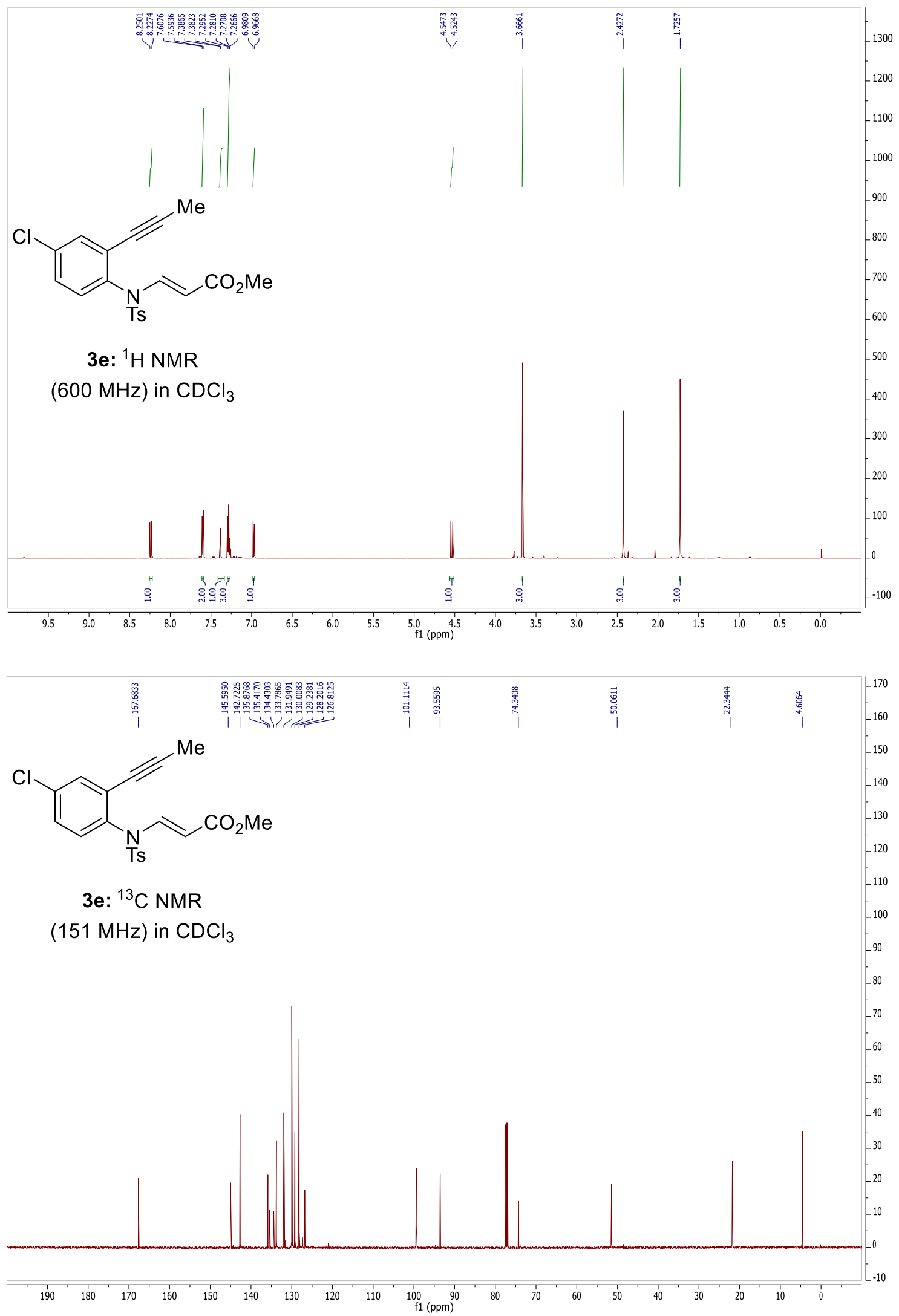


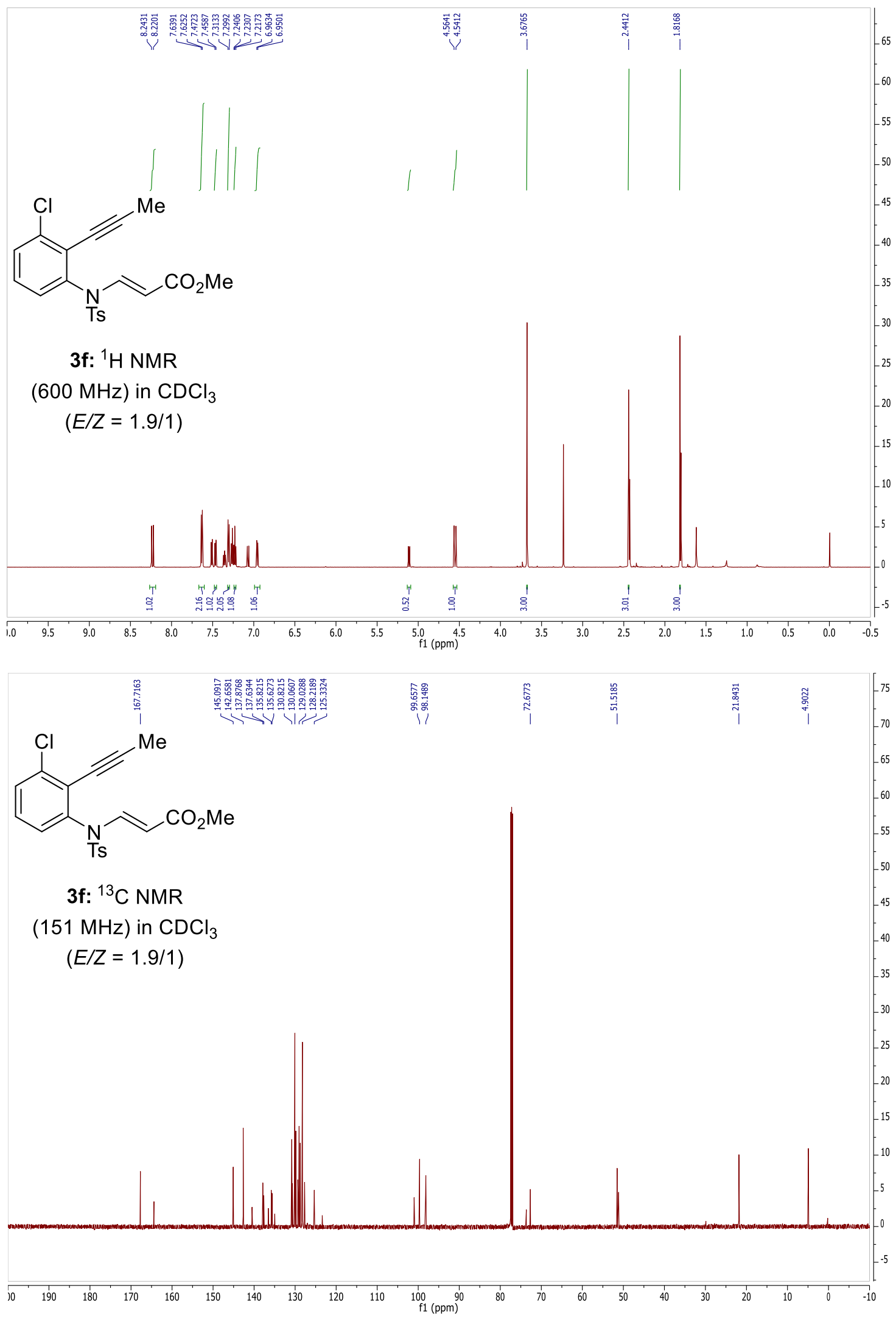



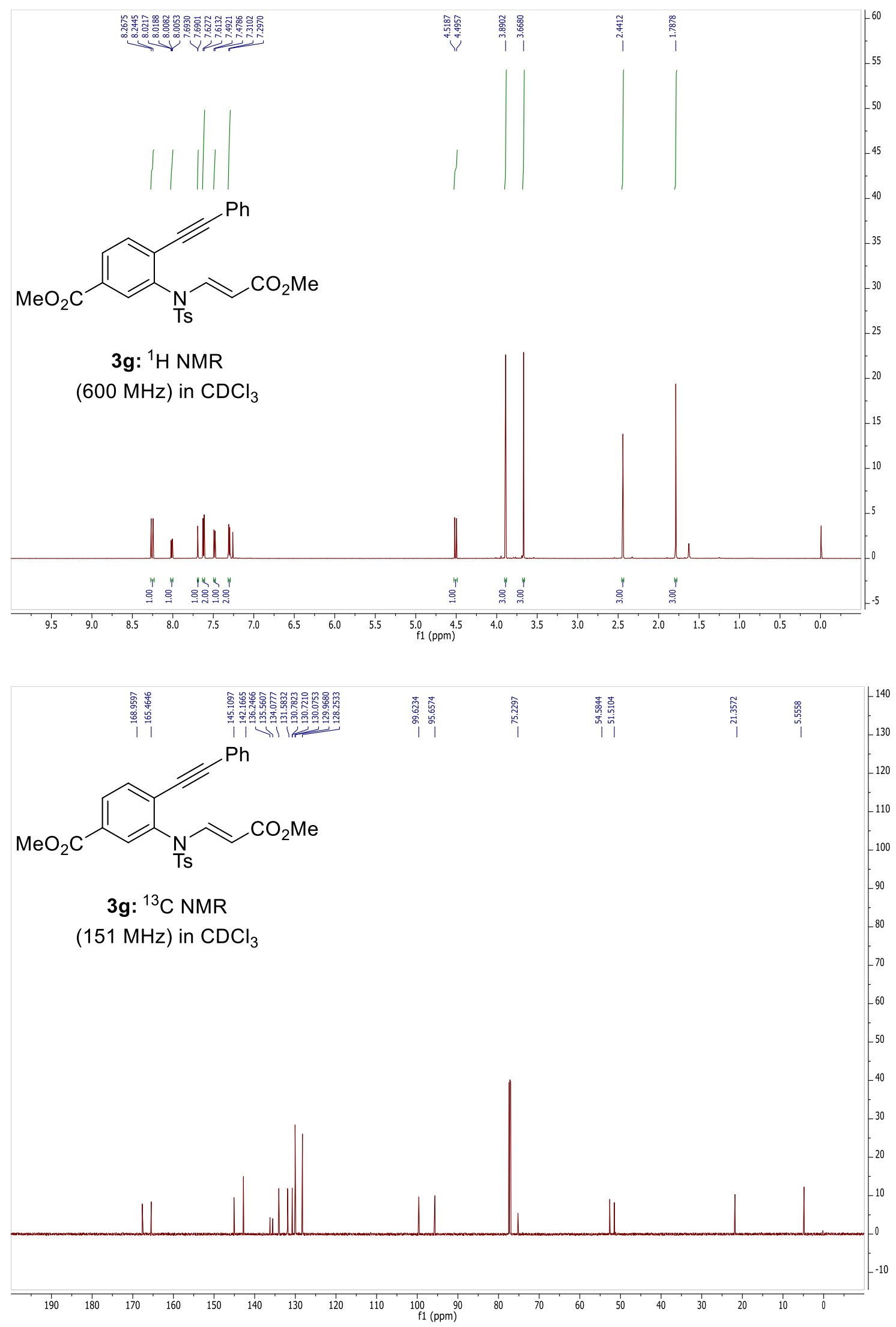

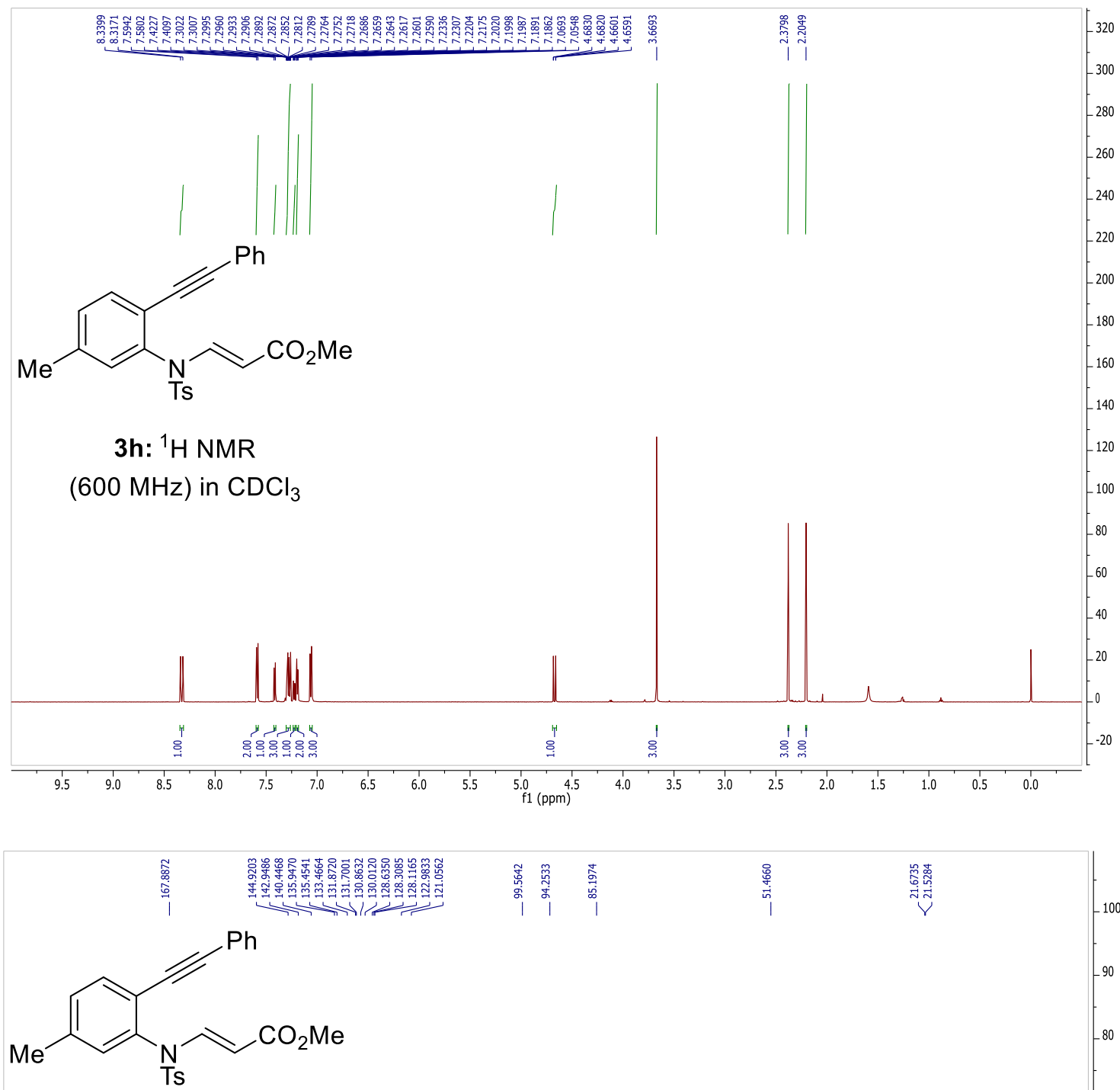

3h: ${ }^{13} \mathrm{C}$ NMR

$(151 \mathrm{MHz})$ in $\mathrm{CDCl}_{3}$

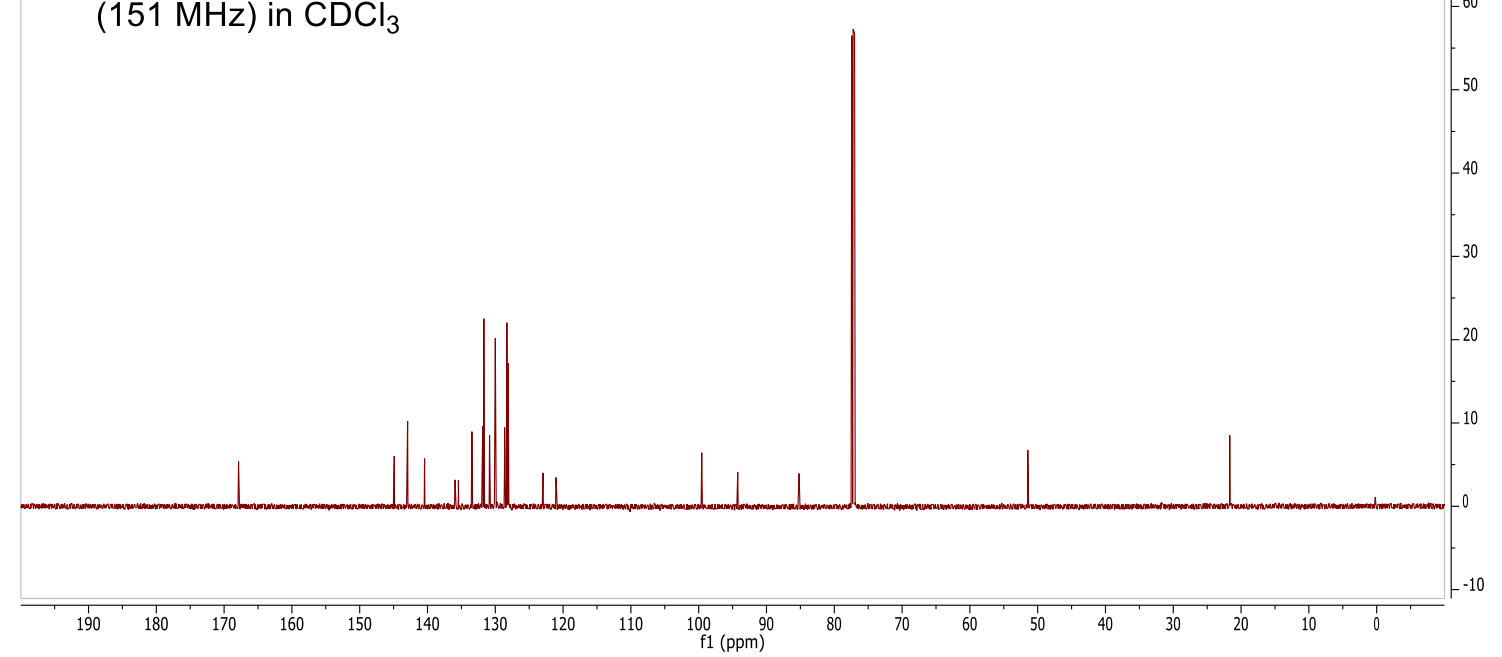



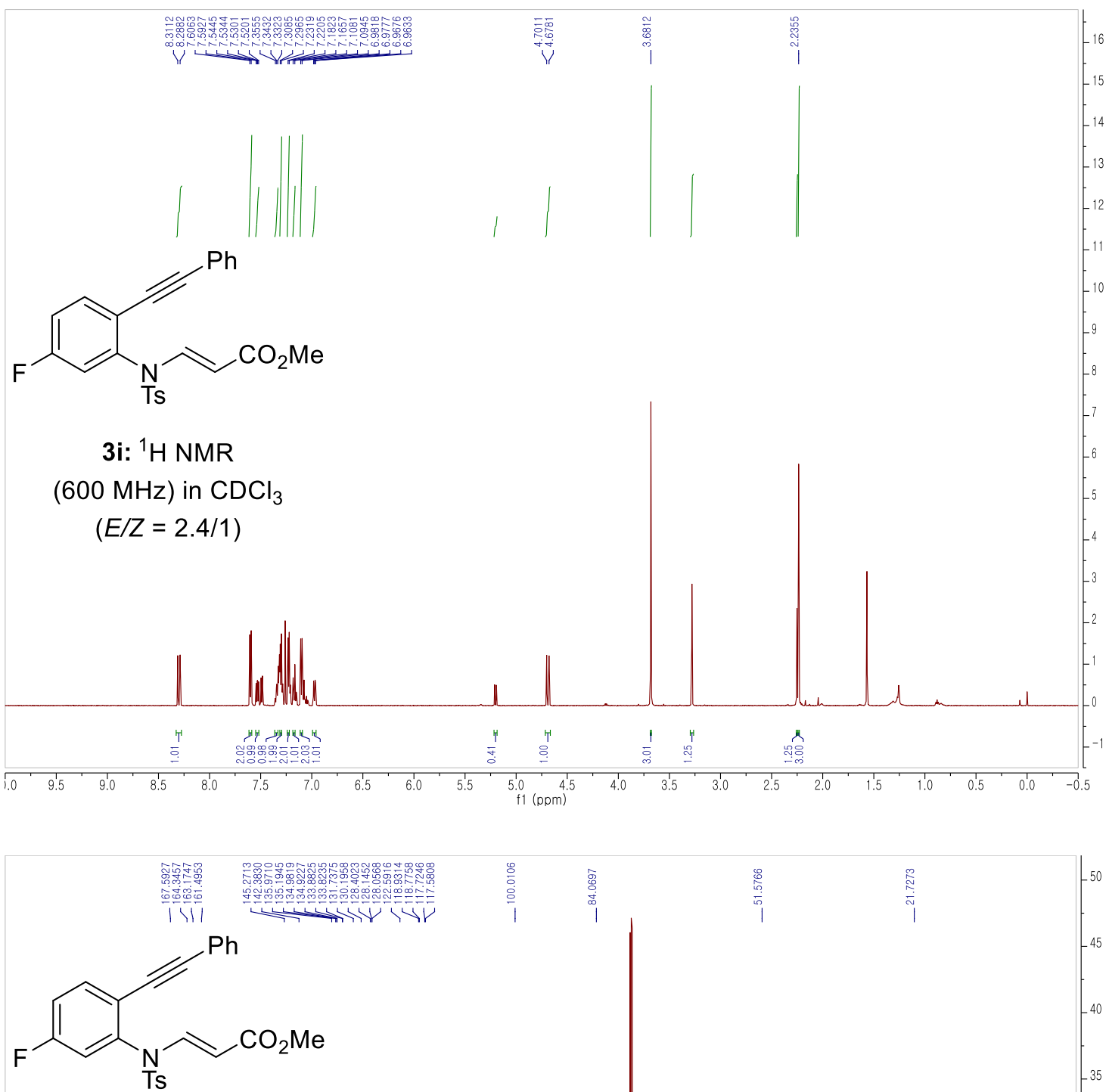

3i: ${ }^{13} \mathrm{C}$ NMR

$(151 \mathrm{MHz})$ in $\mathrm{CDCl}_{3}$

$(E / Z=2.4 / 1)$
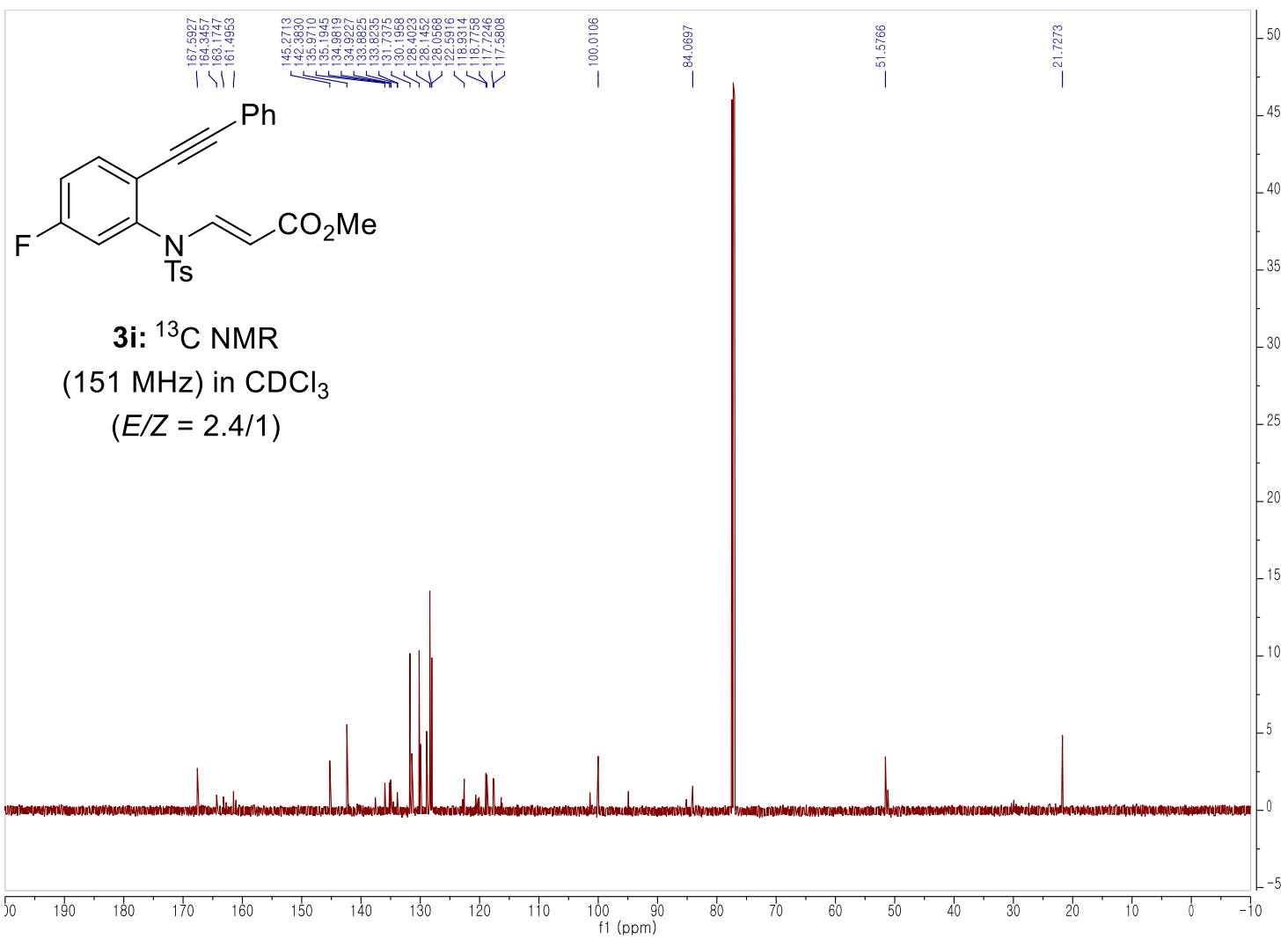


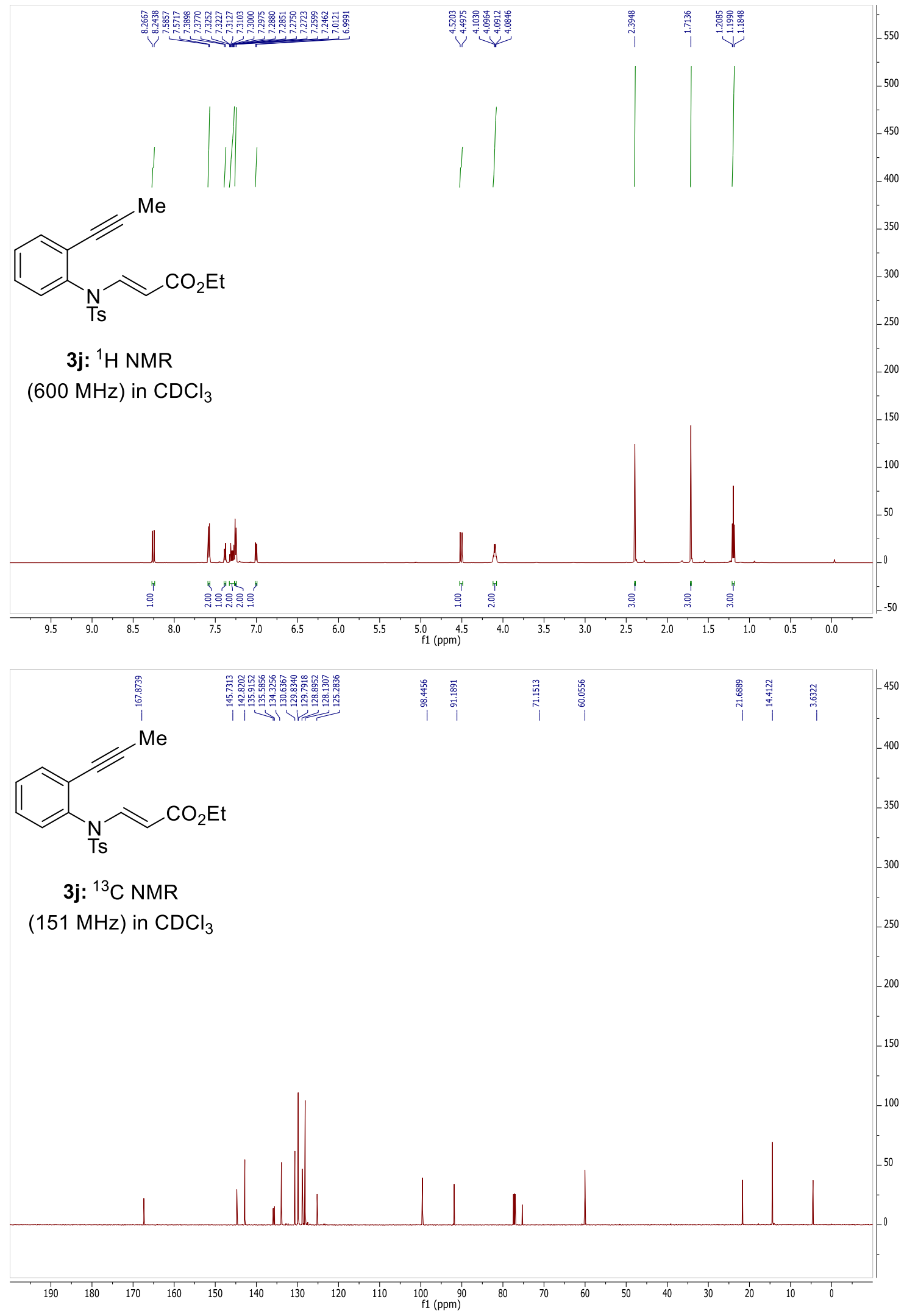



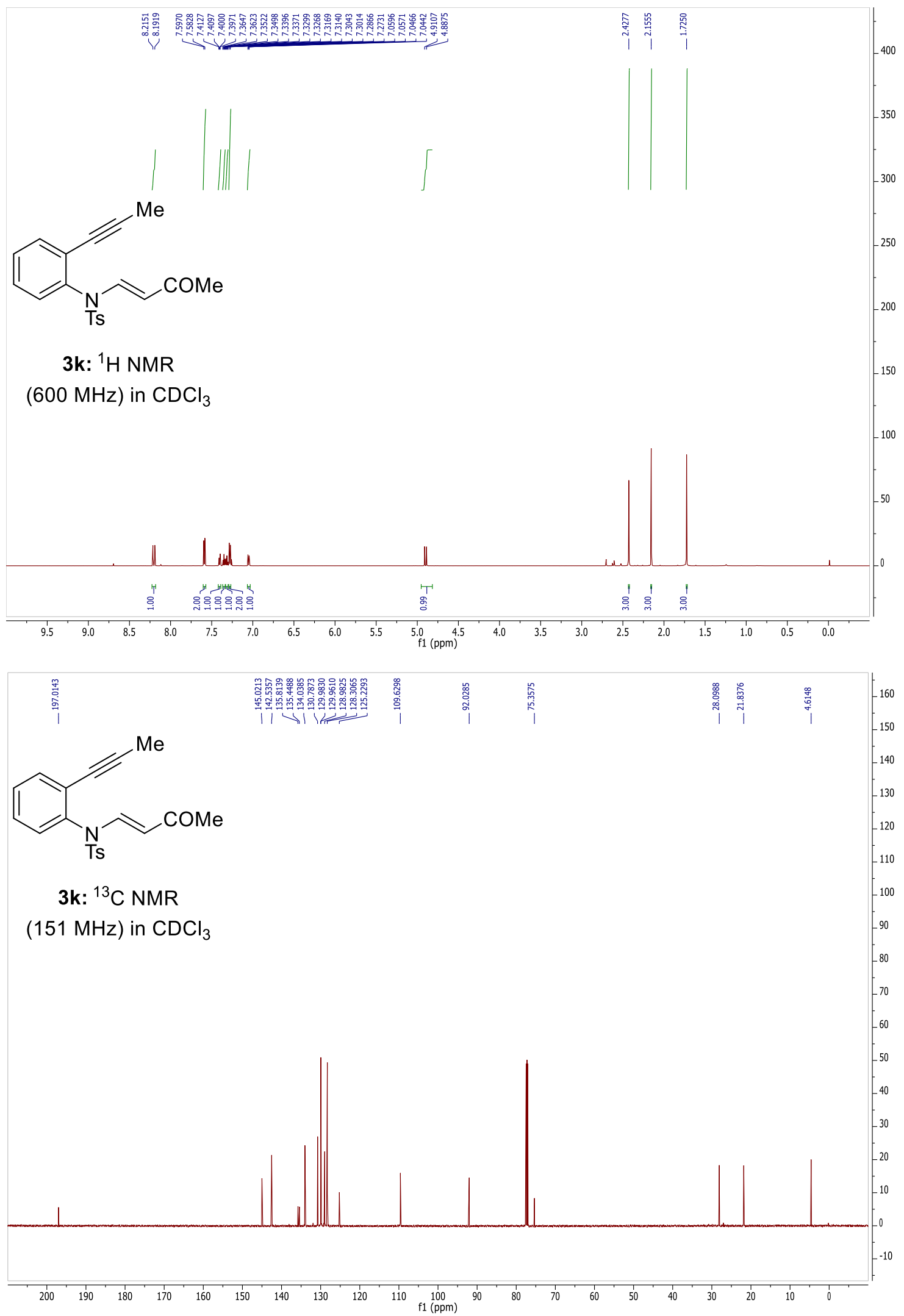

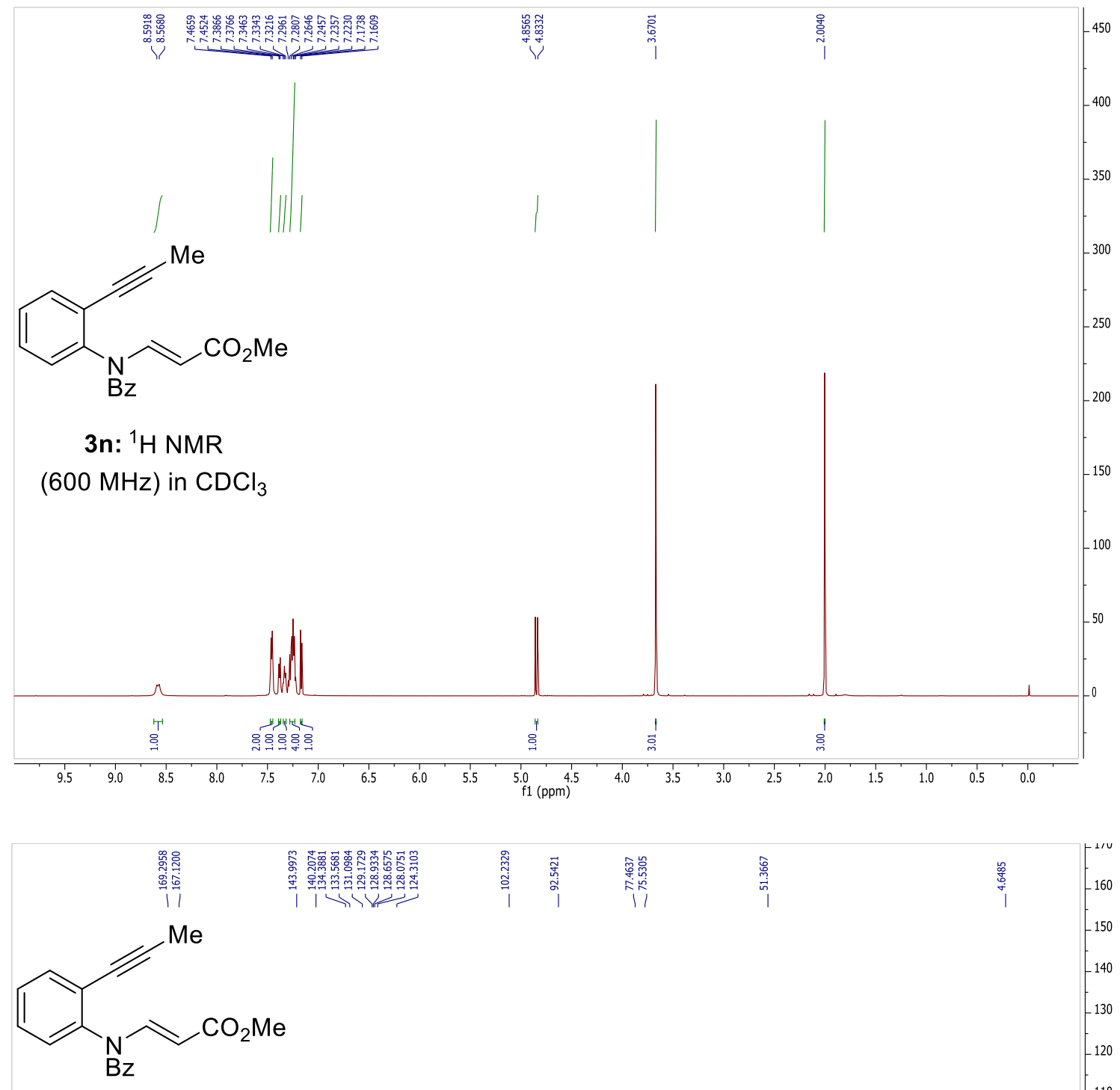

3n: ${ }^{13} \mathrm{C}$ NMR

$(151 \mathrm{MHz})$ in $\mathrm{CDCl}_{3}$

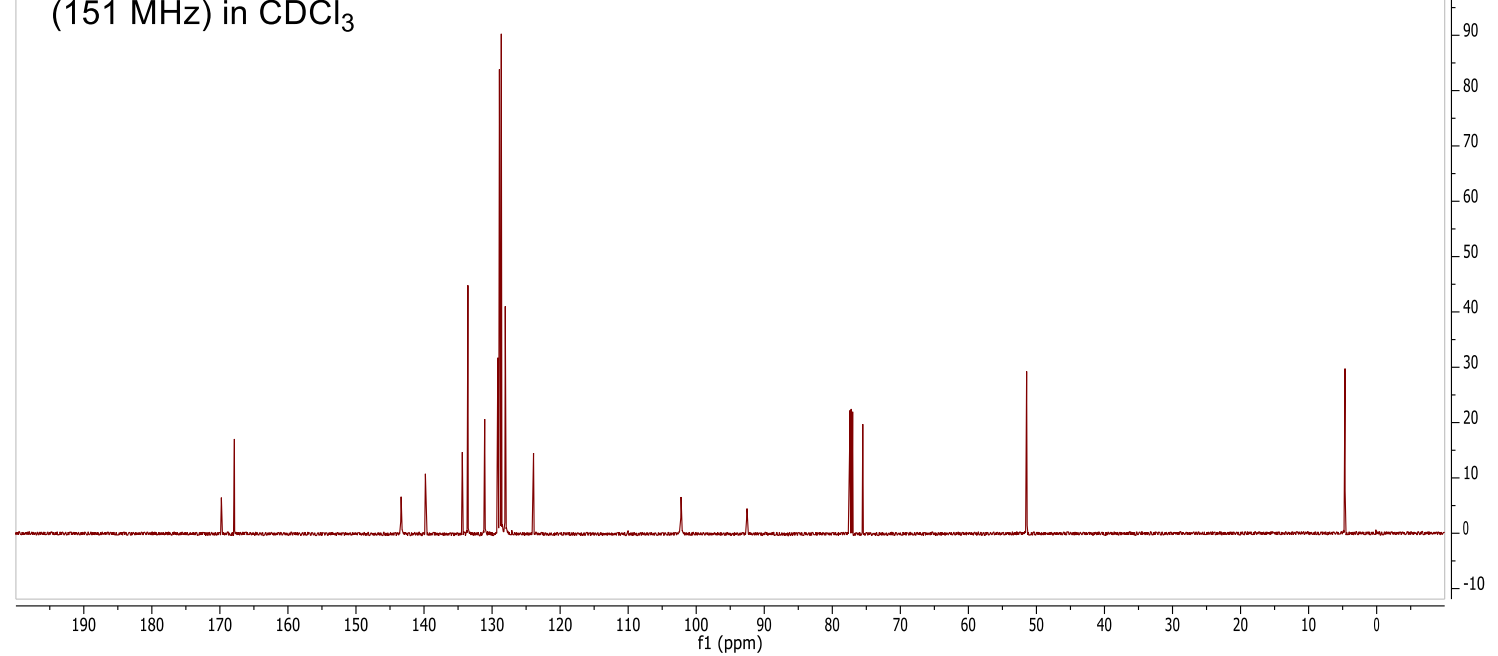



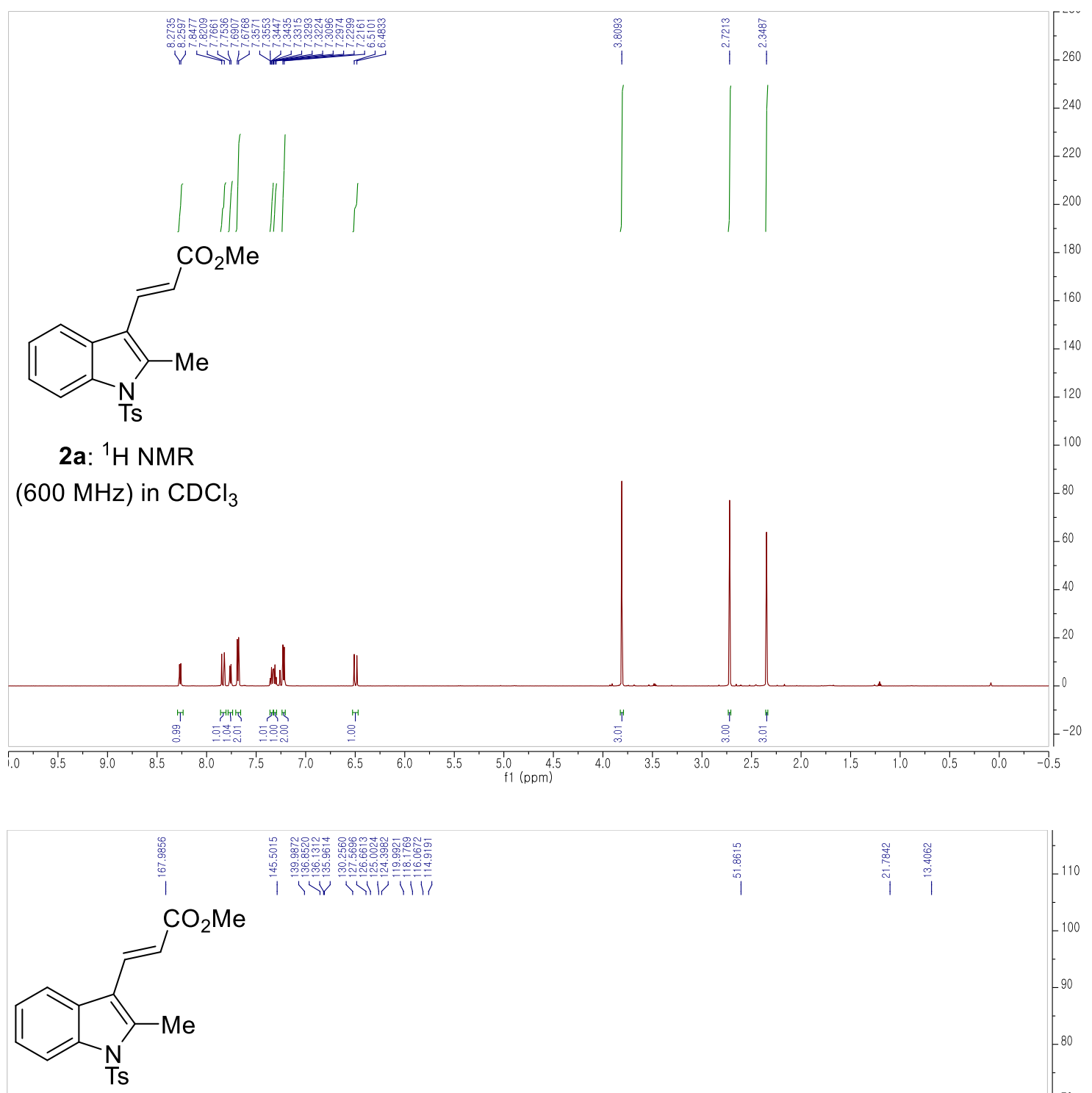

2a: ${ }^{13} \mathrm{C}$ NMR

$(151 \mathrm{MHz})$ in $\mathrm{CDCl}_{3}$

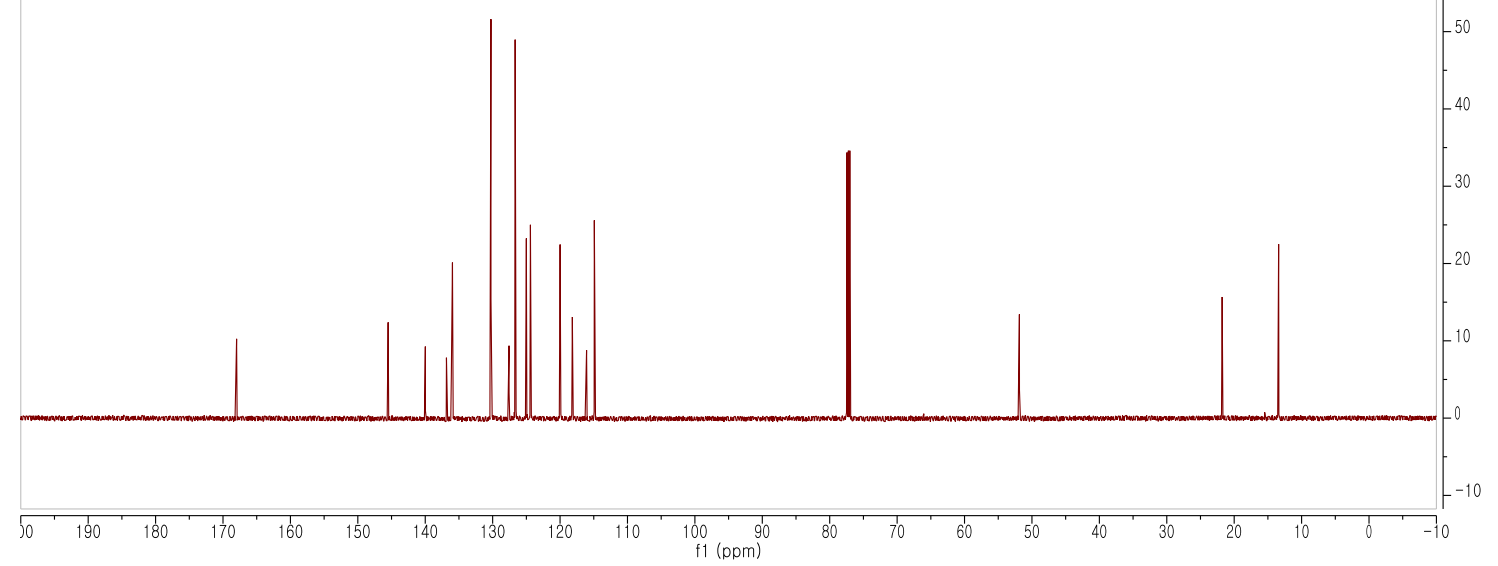




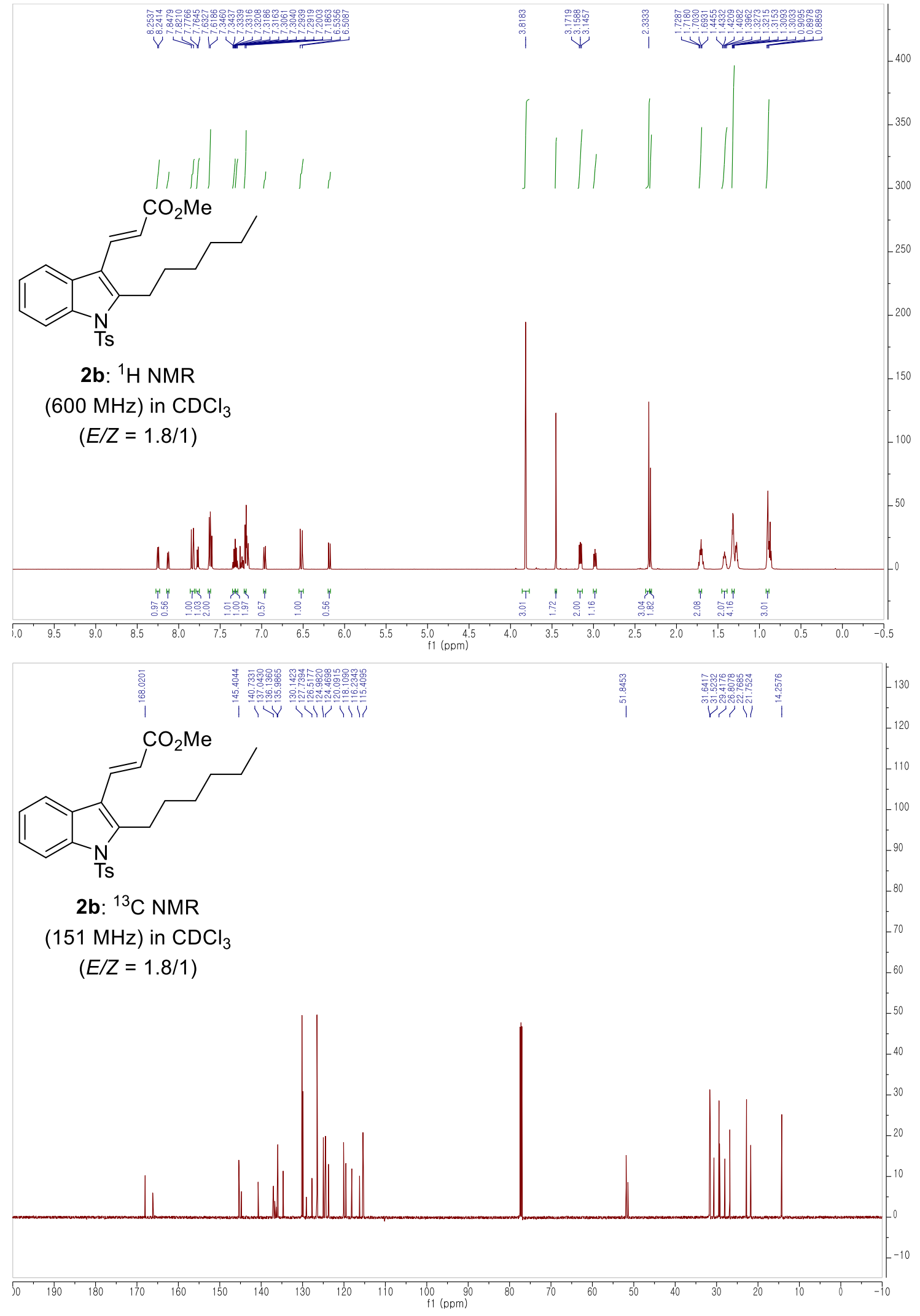



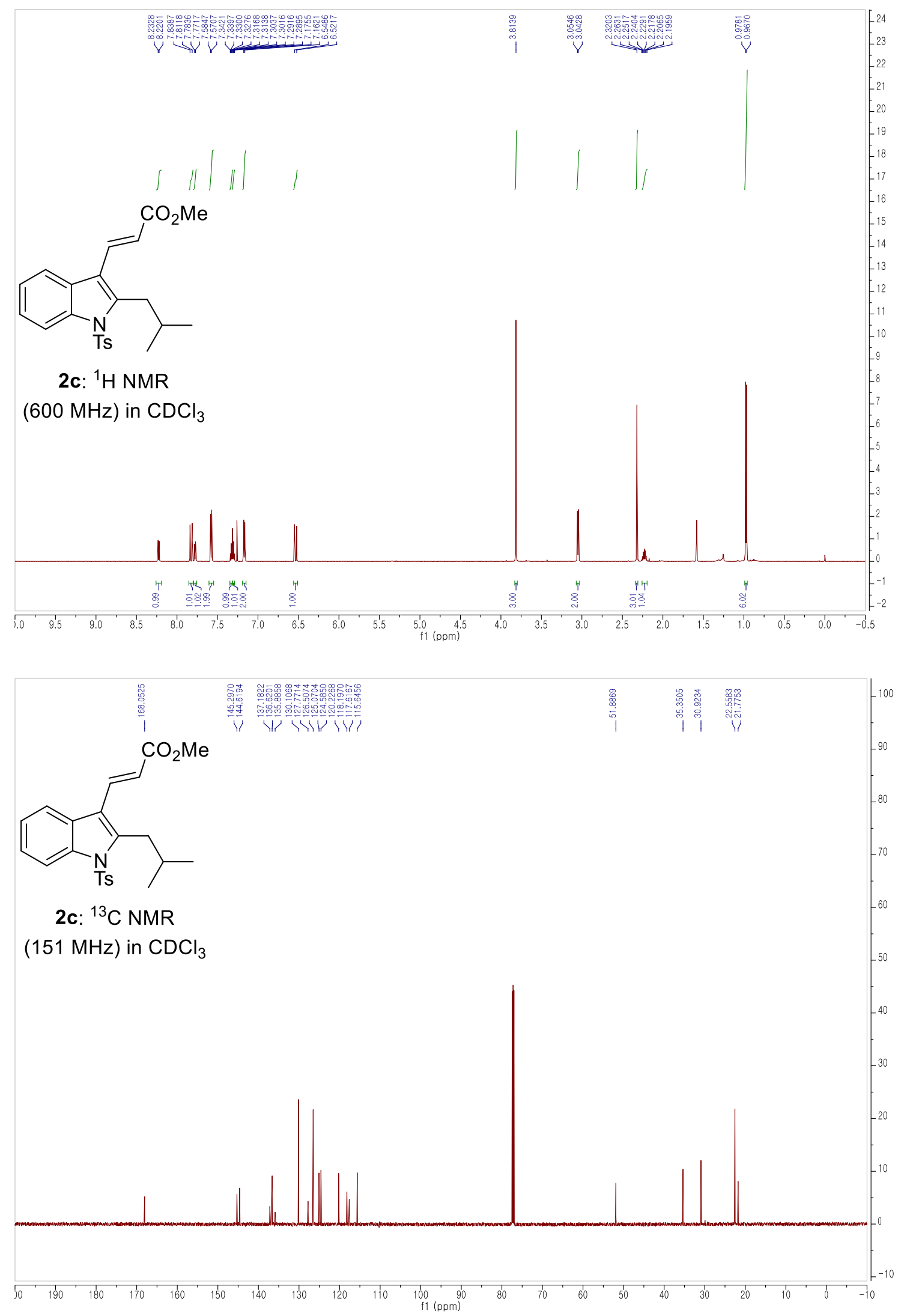

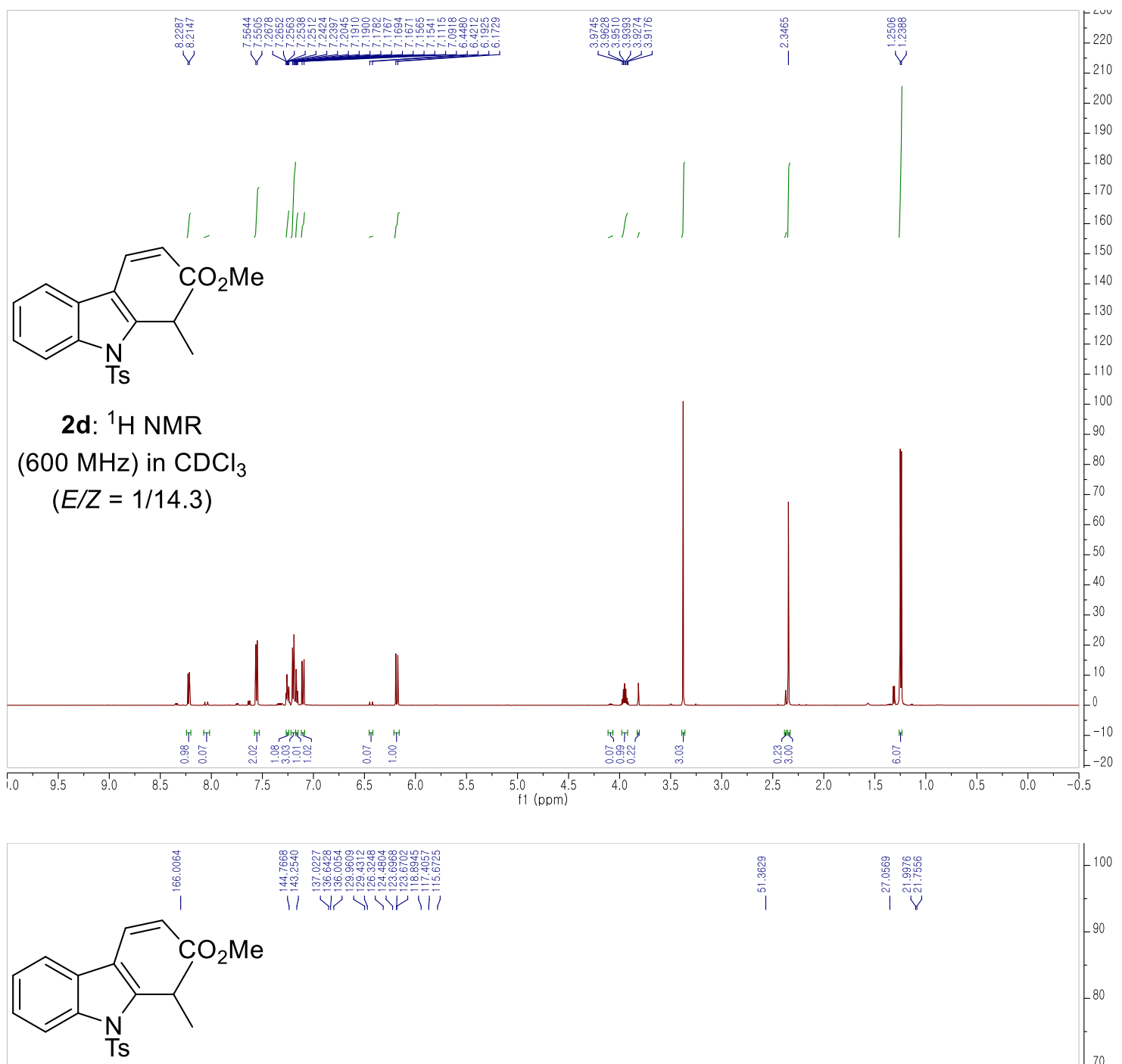

2d: ${ }^{13} \mathrm{C}$ NMR

$(151 \mathrm{MHz})$ in $\mathrm{CDCl}_{3}$

$(E / Z=1 / 14.3)$

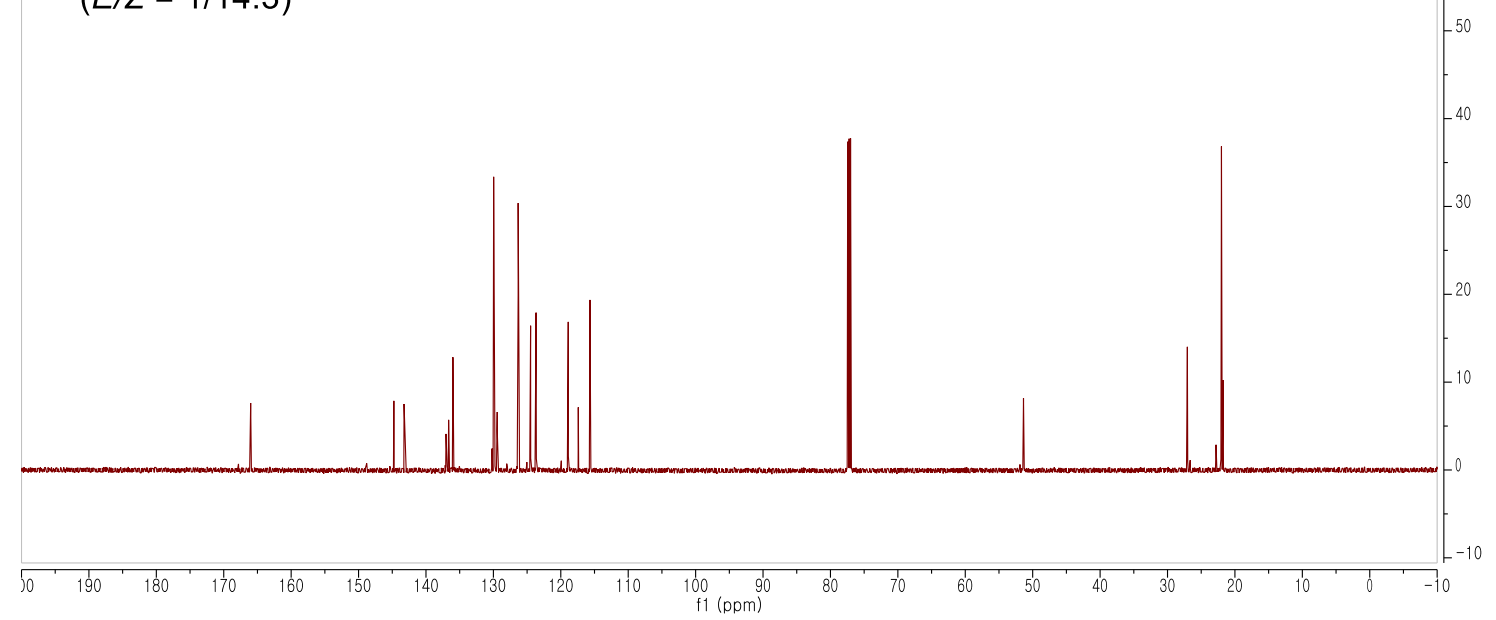




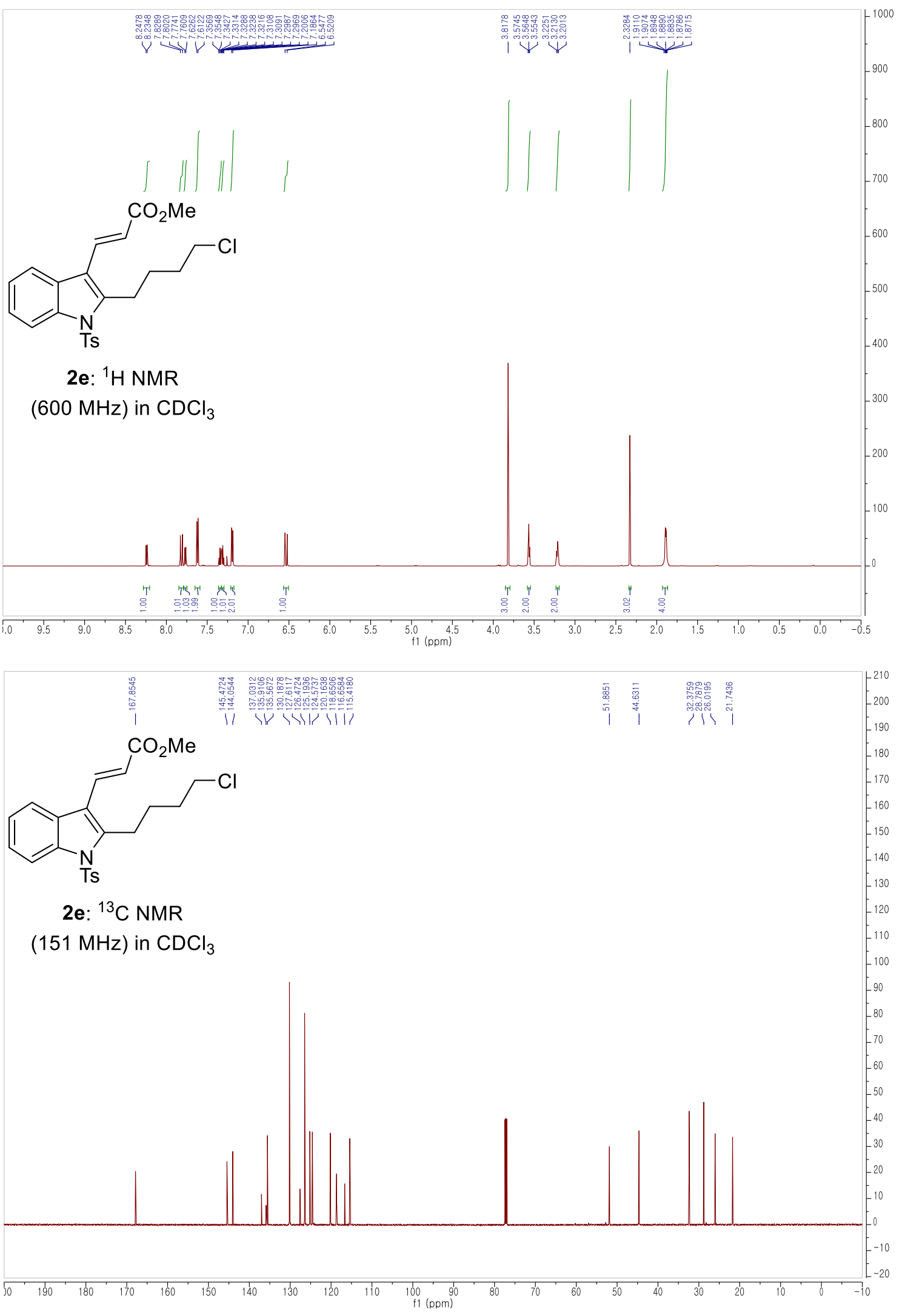



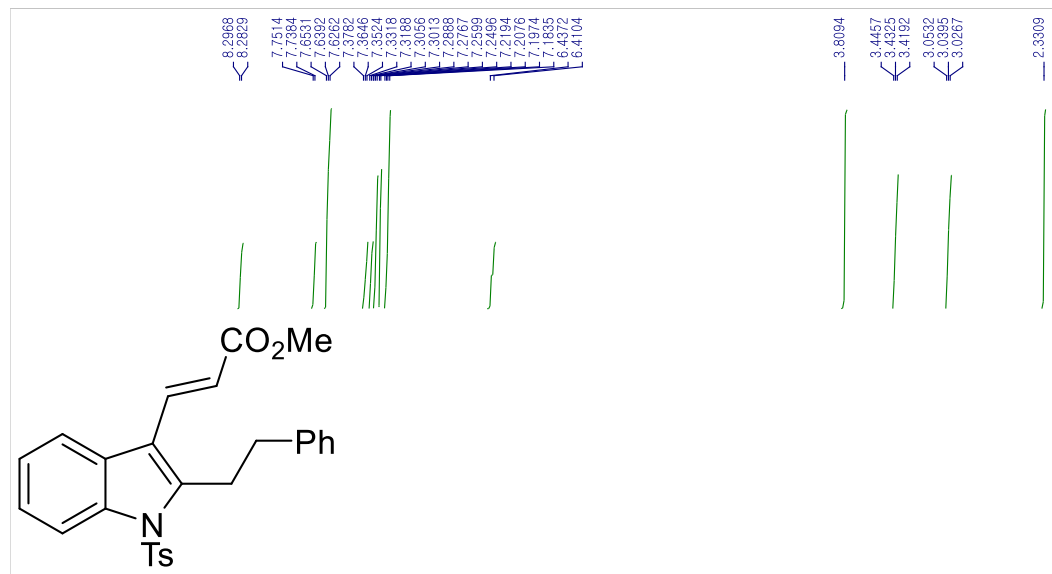

2f: ${ }^{1} \mathrm{H}$ NMR (600 MHz) in $\mathrm{CDCl}_{3}$
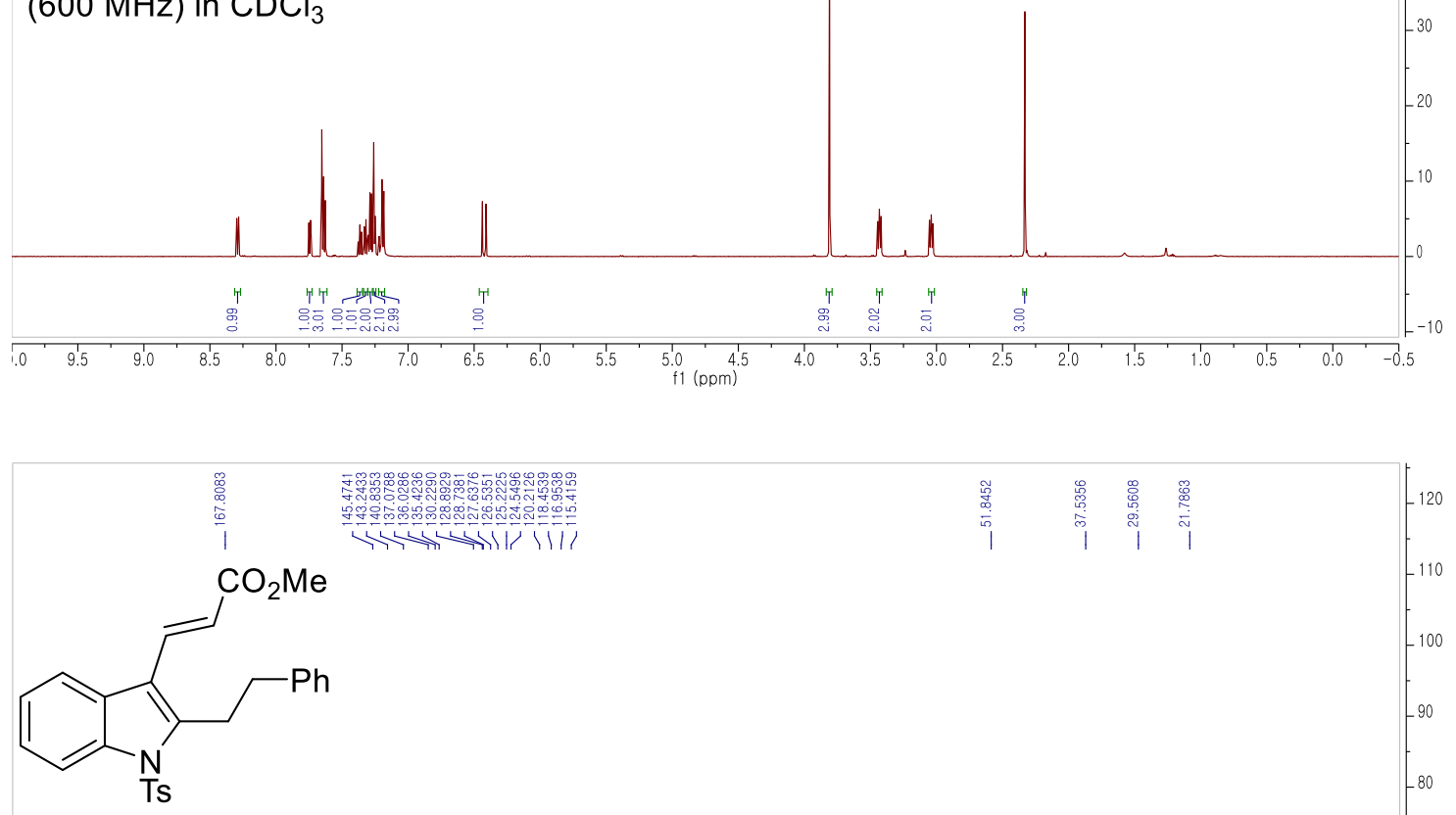

2f: ${ }^{13} \mathrm{C}$ NMR $(151 \mathrm{MHz})$ in $\mathrm{CDCl}_{3}$

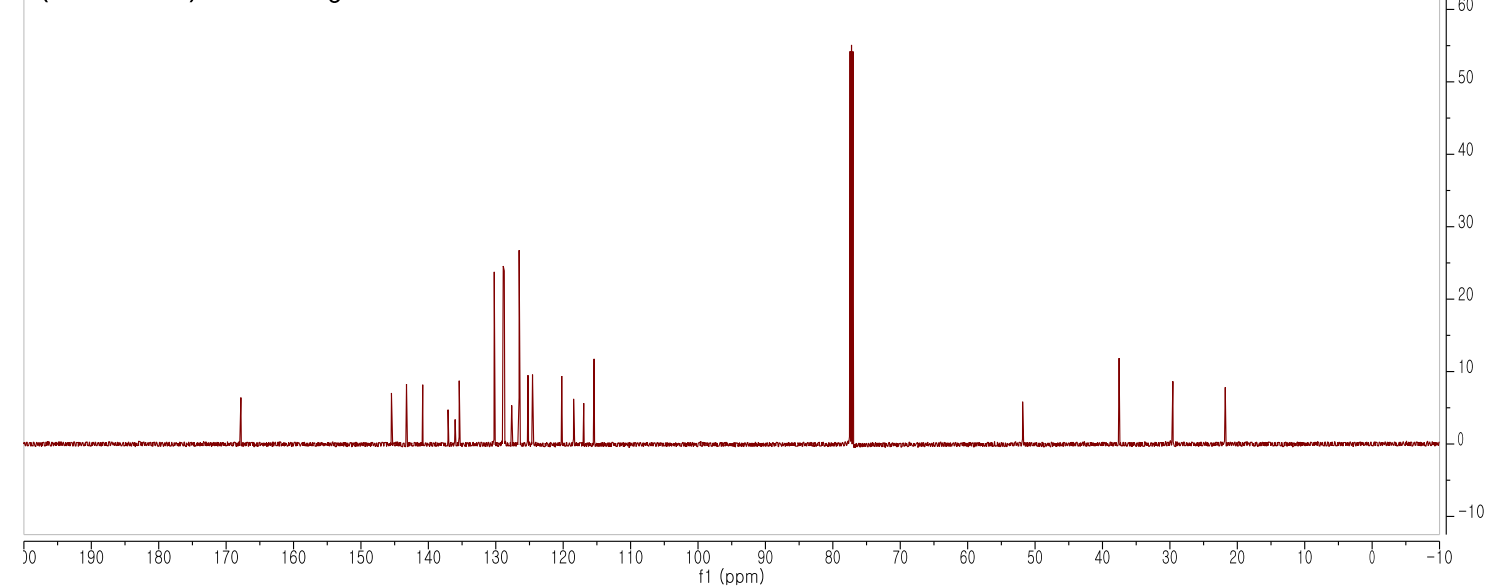




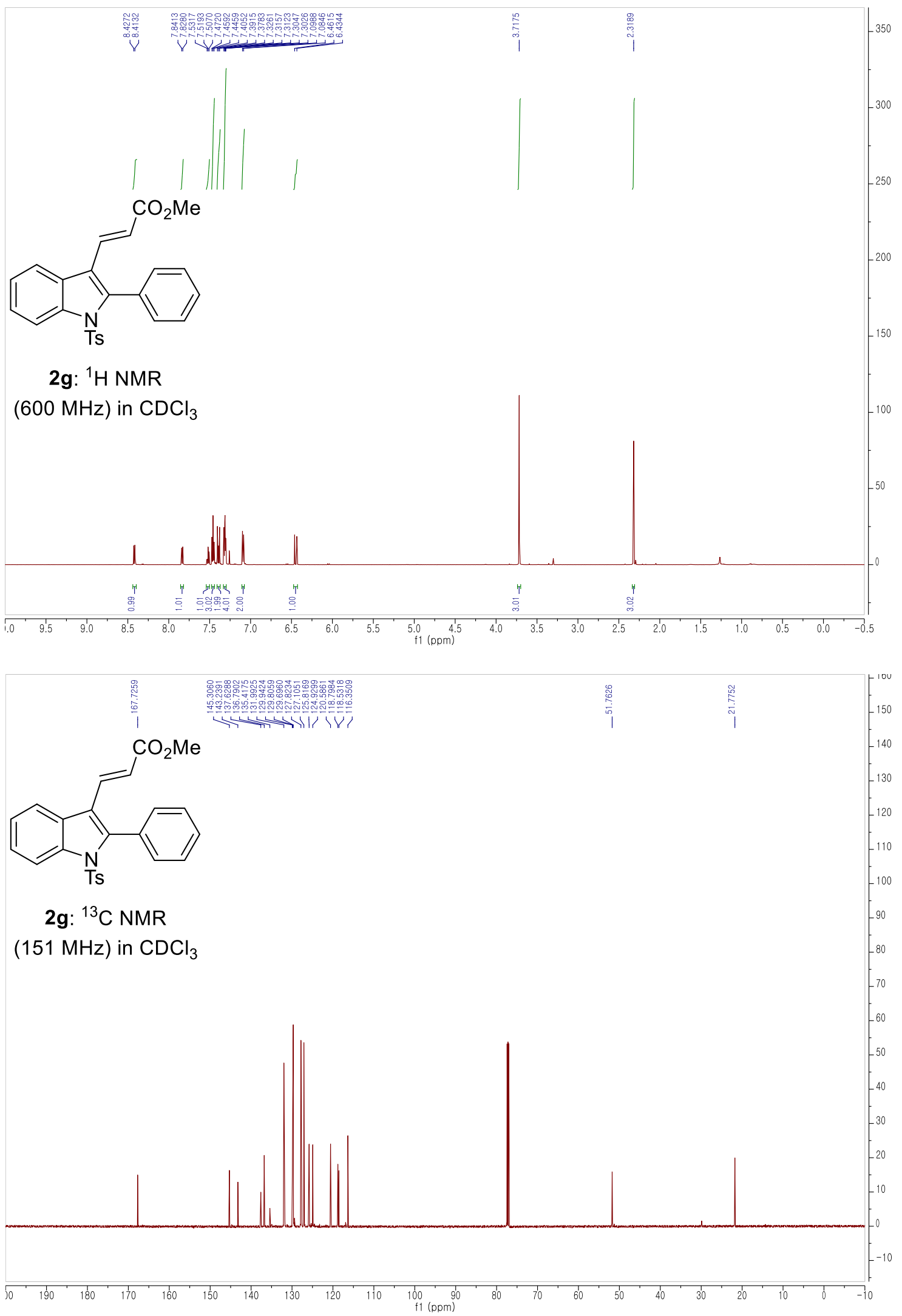




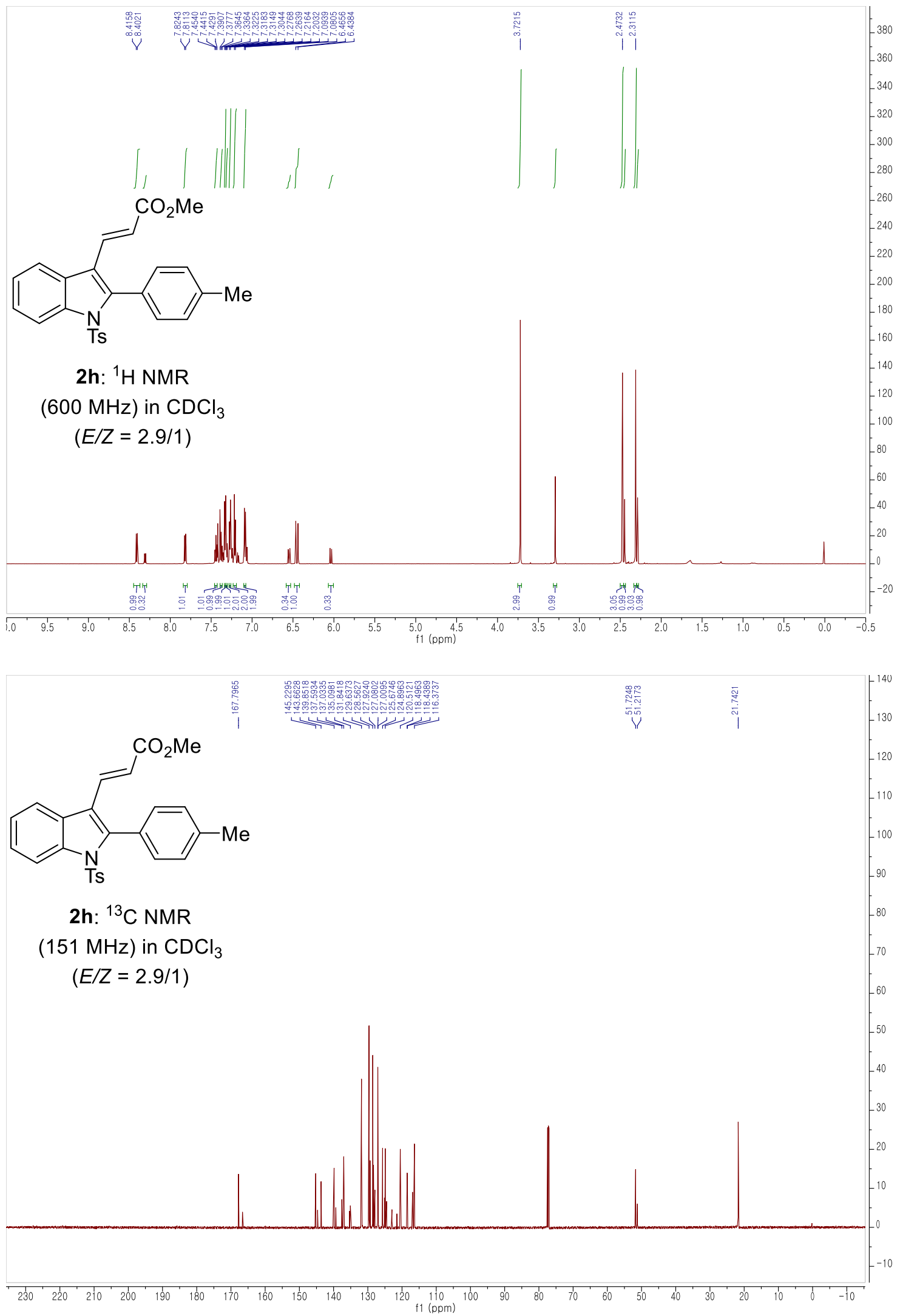



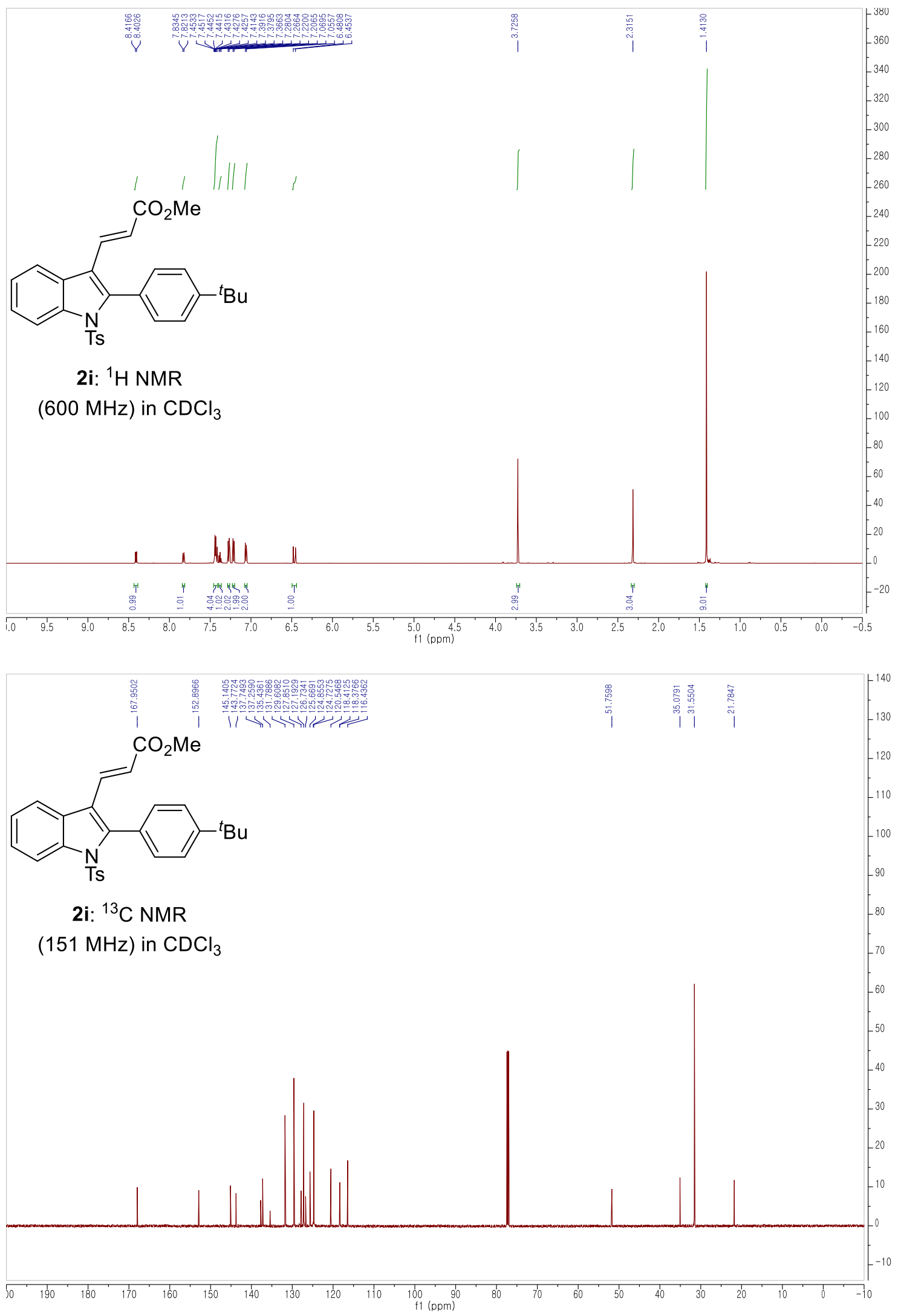


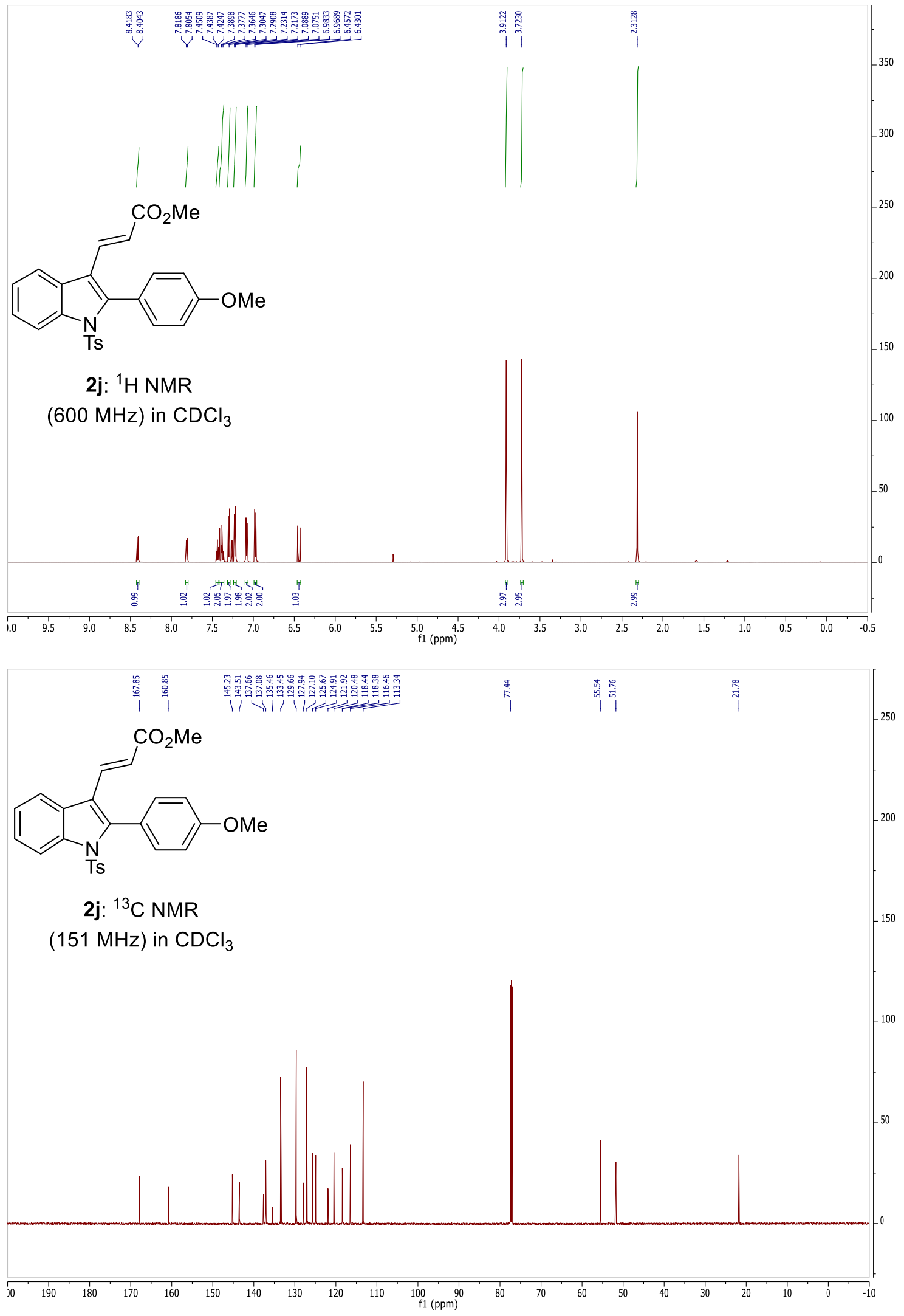



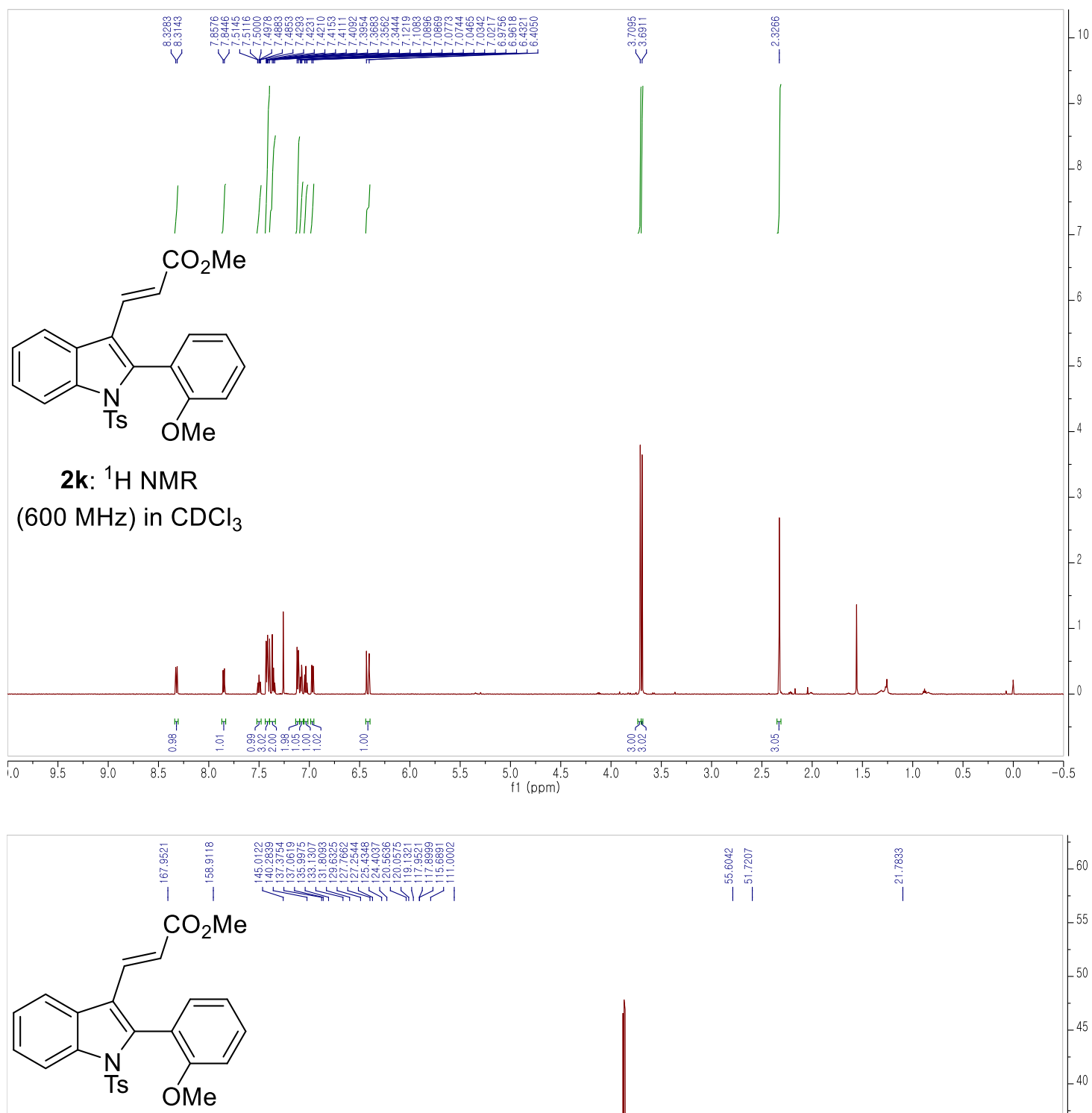

2k: ${ }^{13} \mathrm{C}$ NMR

$(151 \mathrm{MHz})$ in $\mathrm{CDCl}_{3}$

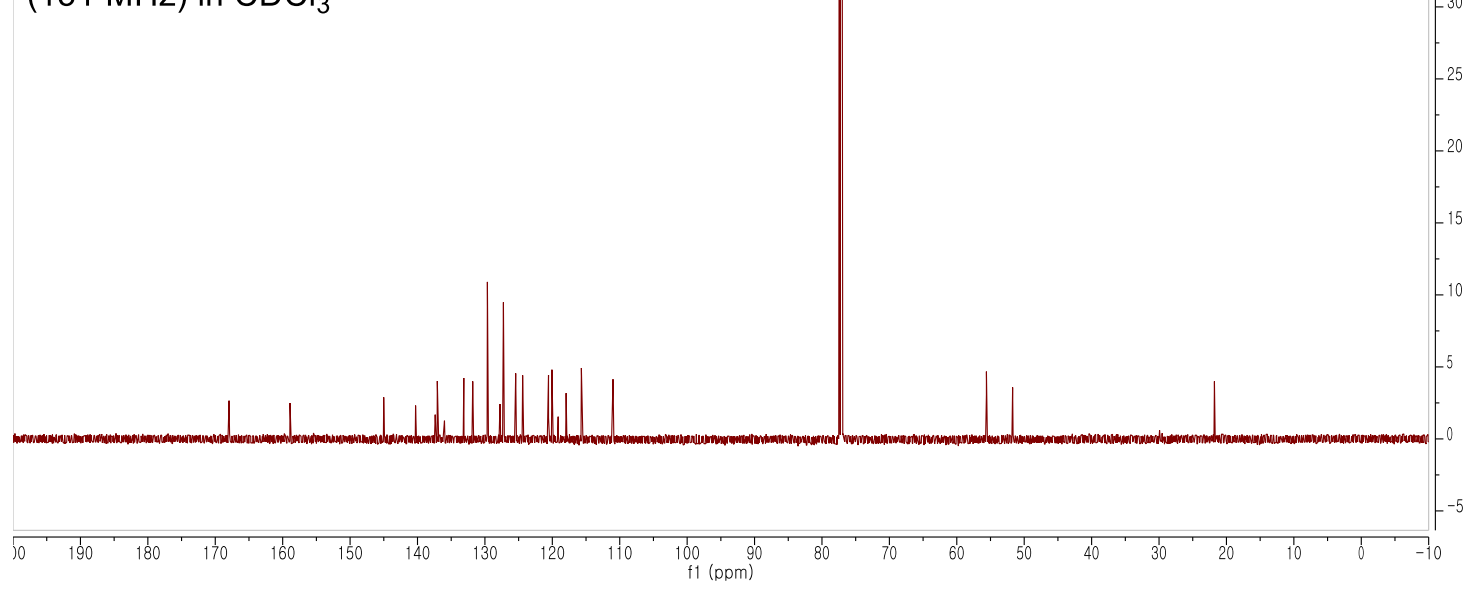




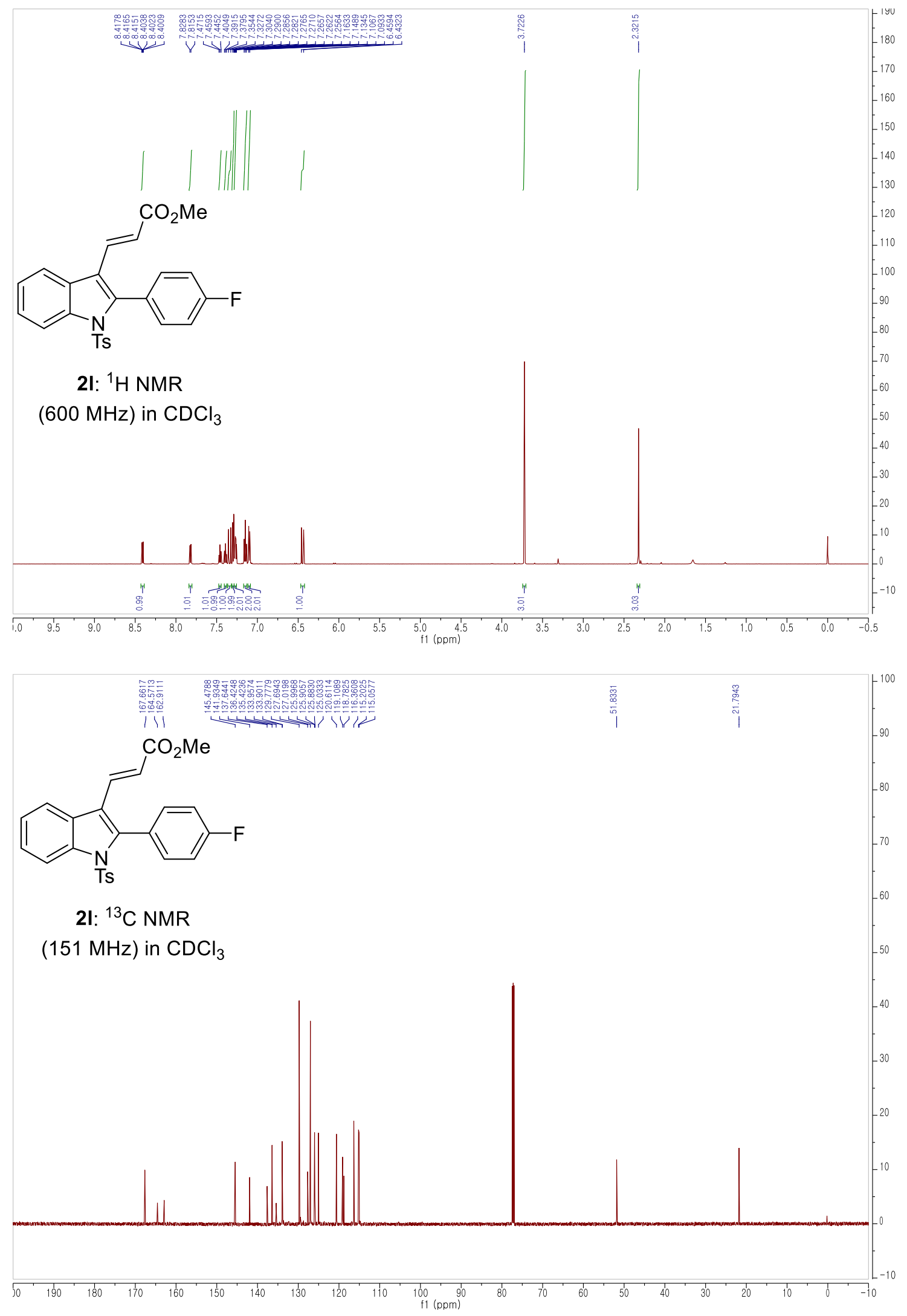



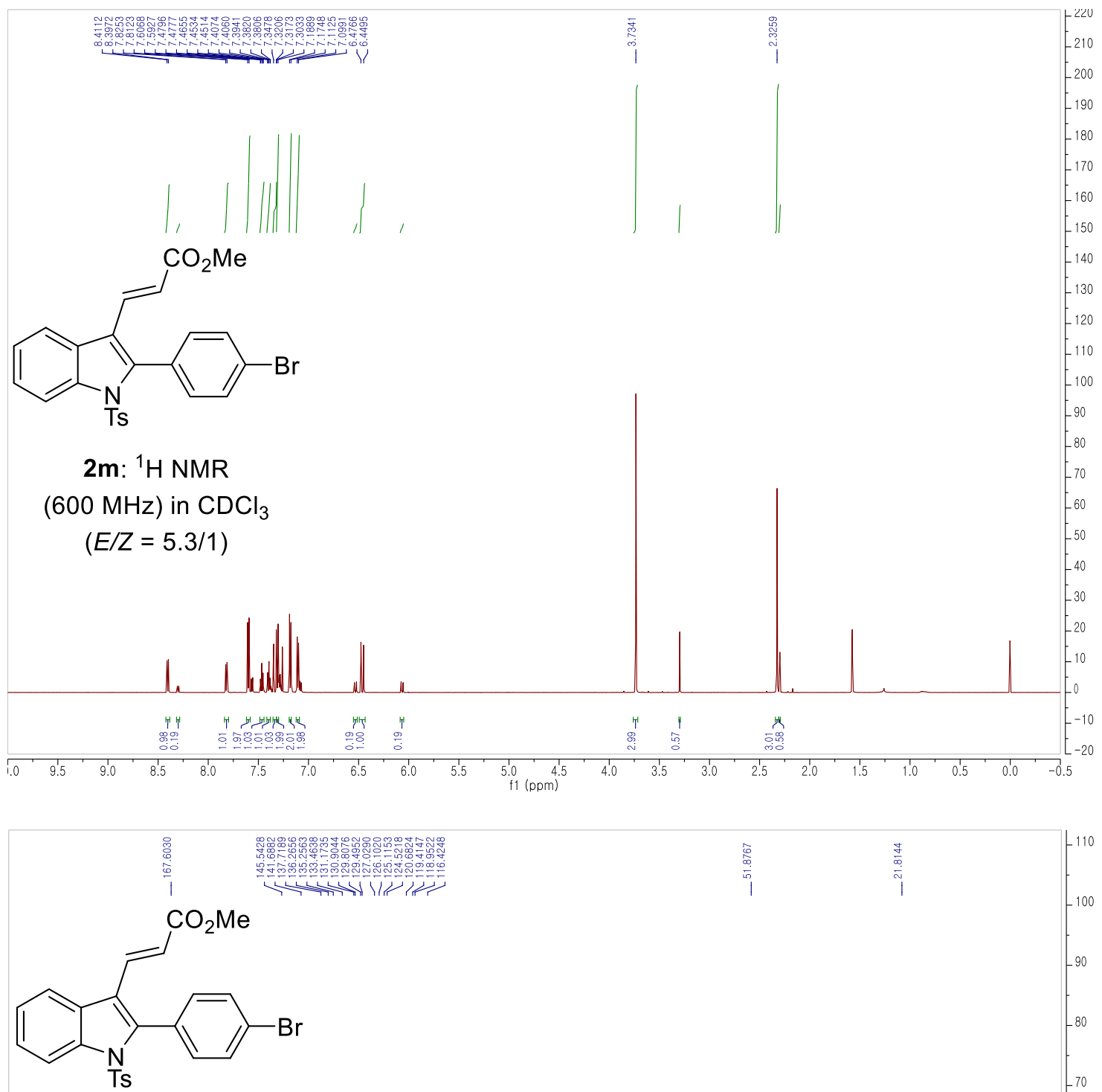

2m: ${ }^{13} \mathrm{C}$ NMR

$(151 \mathrm{MHz})$ in $\mathrm{CDCl}_{3}$

$(E / Z=5.3 / 1)$

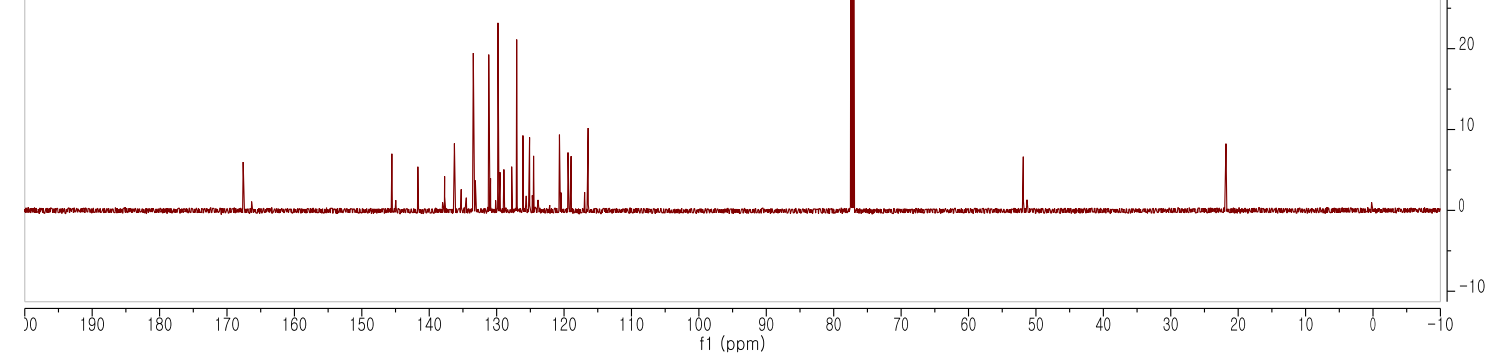



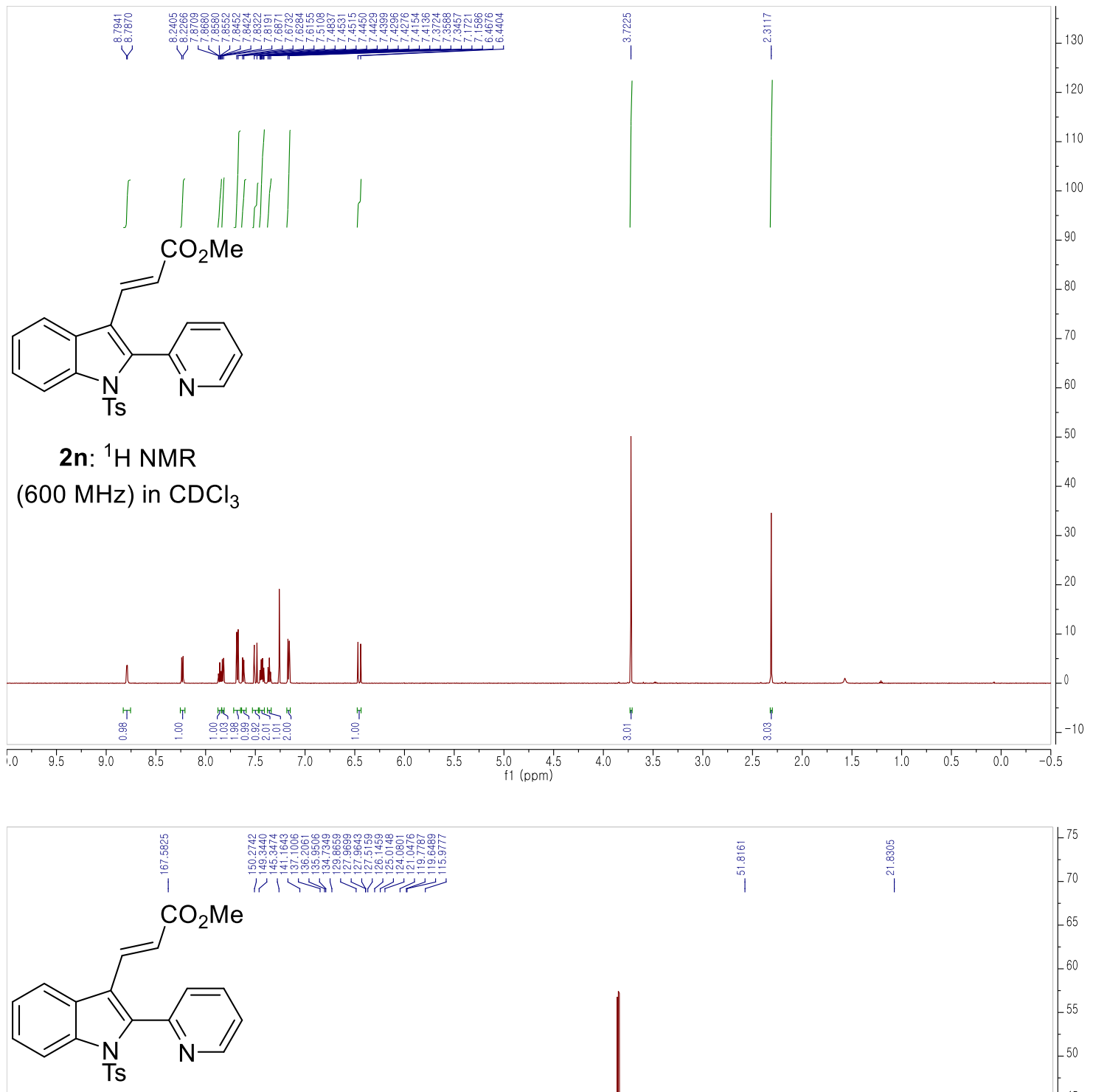

2n: ${ }^{13} \mathrm{C}$ NMR

$(151 \mathrm{MHz})$ in $\mathrm{CDCl}_{3}$

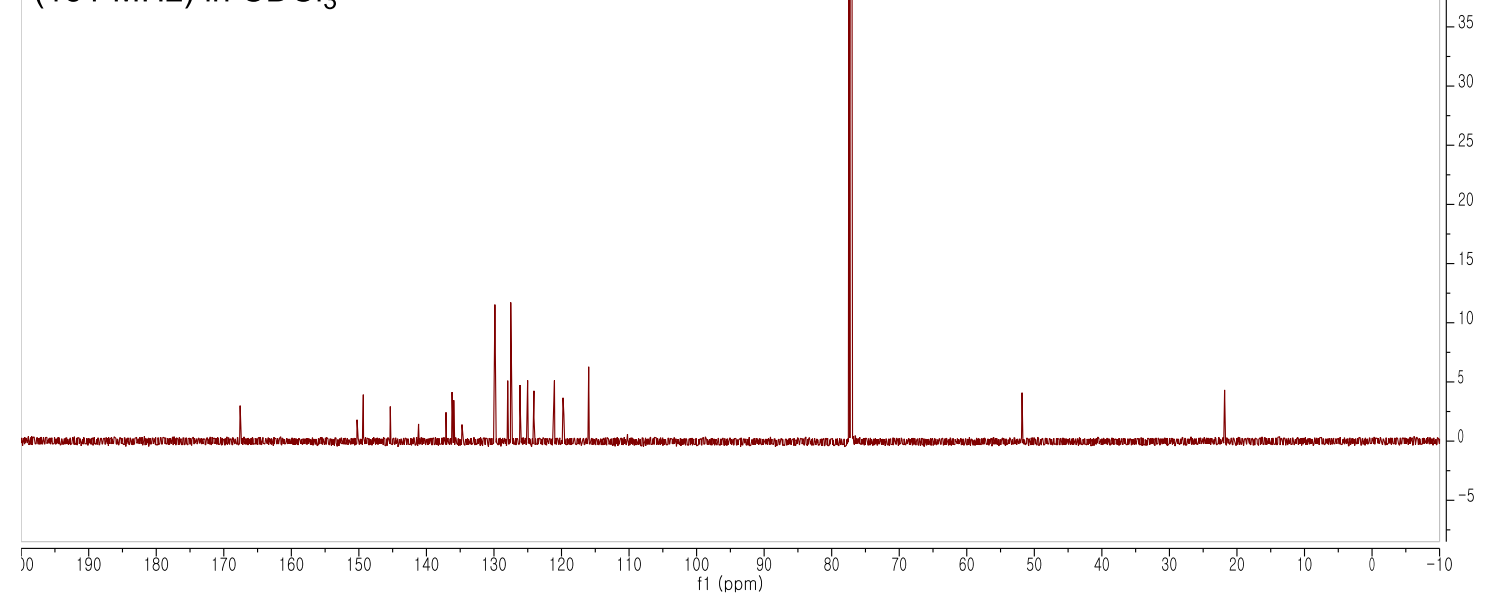



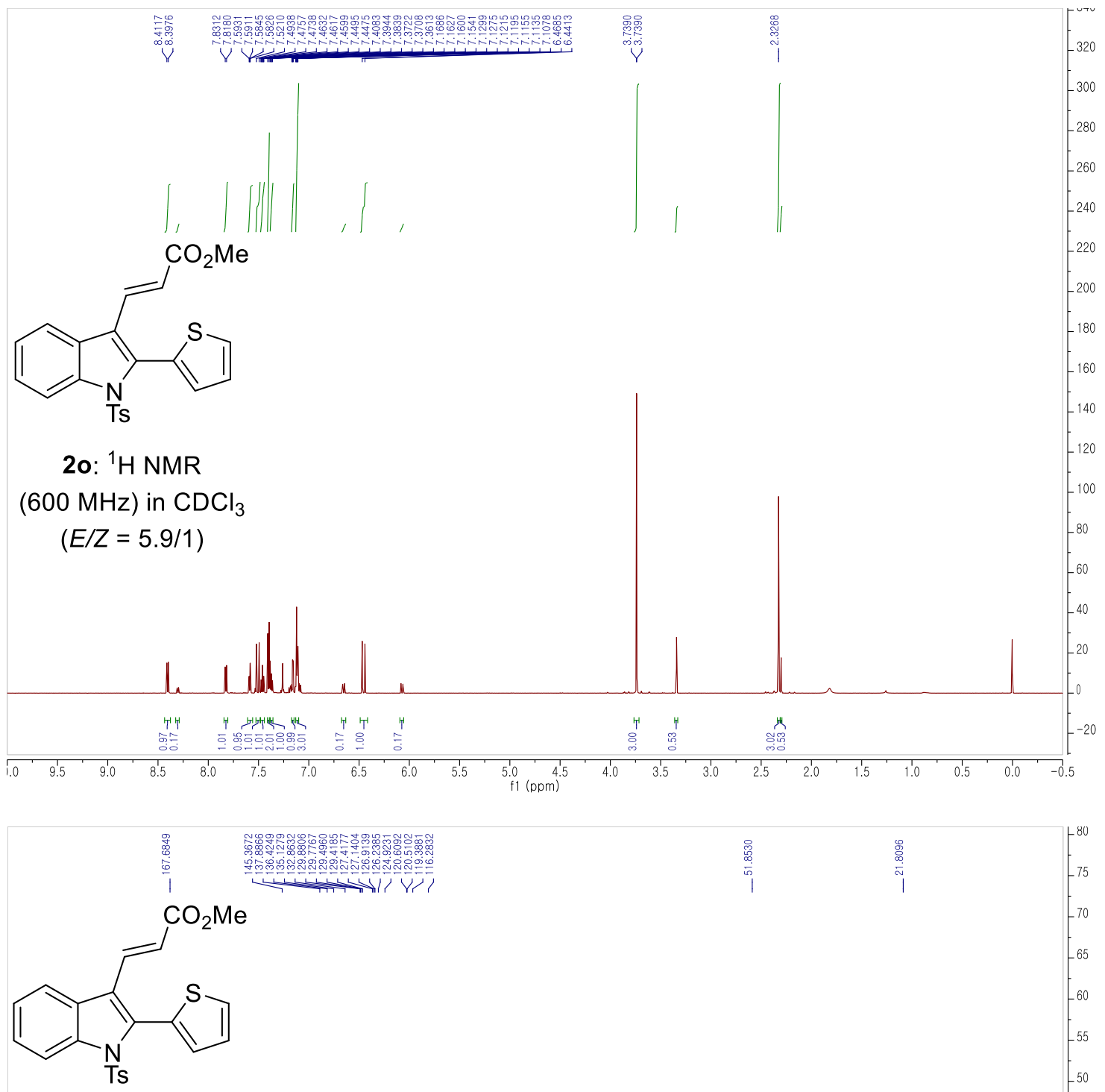

2o: ${ }^{13} \mathrm{C}$ NMR

$(151 \mathrm{MHz})$ in $\mathrm{CDCl}_{3}$

$(E / Z=5.9 / 1)$

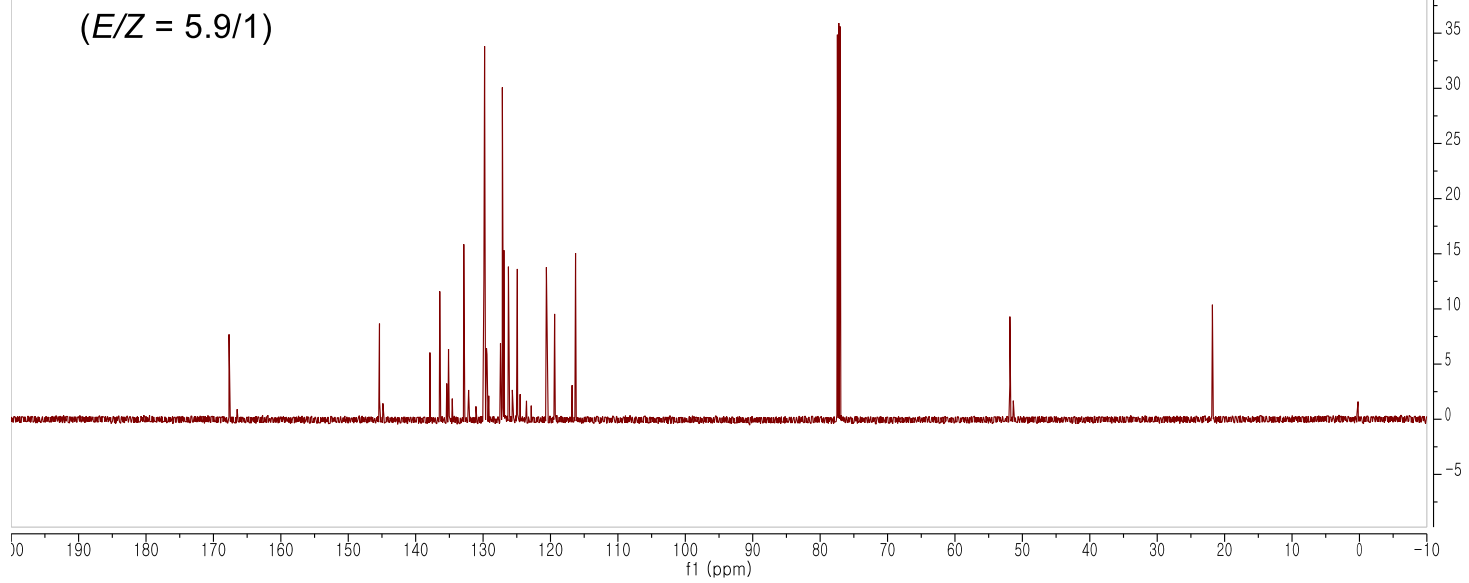



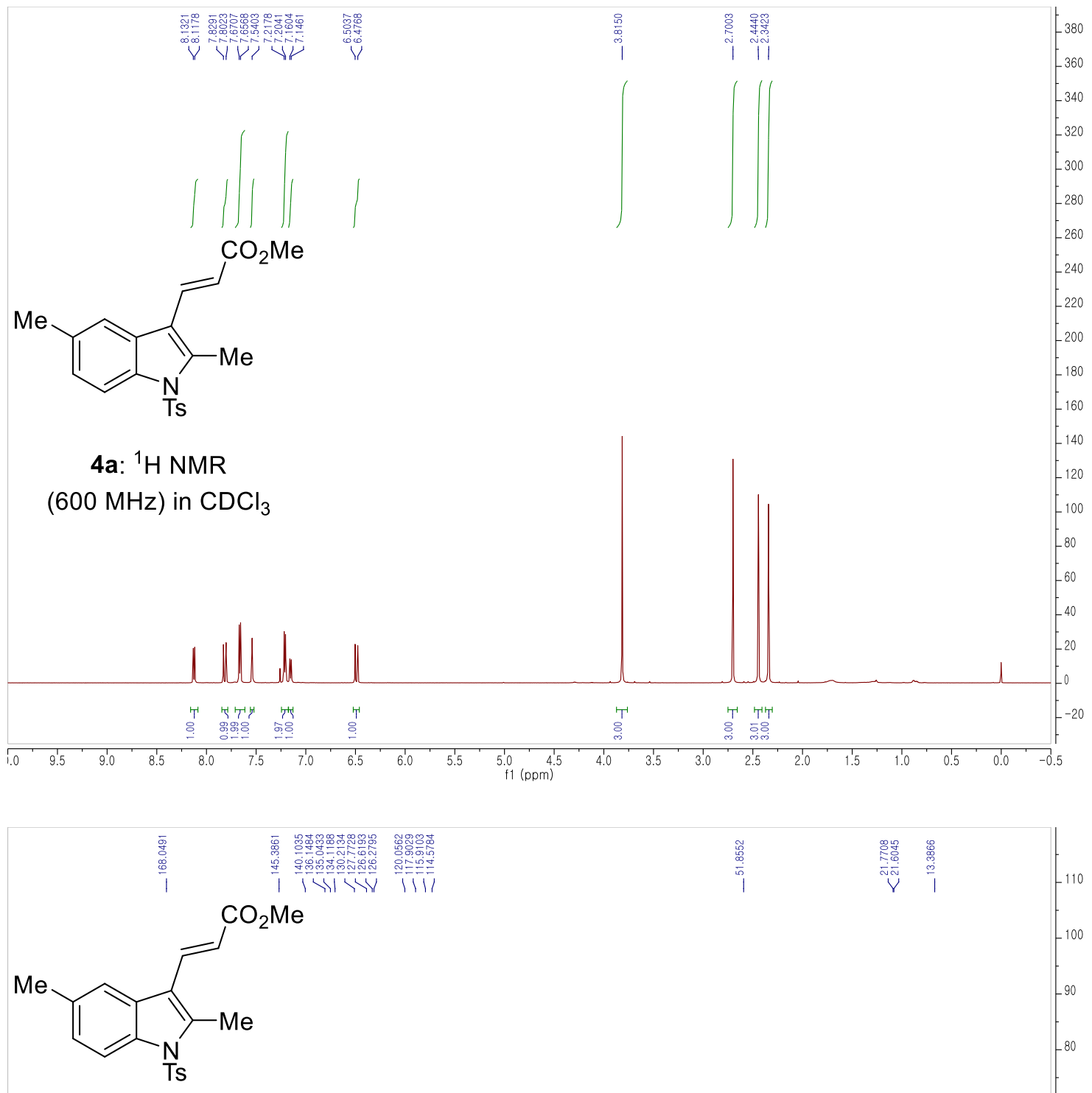

4a: ${ }^{13} \mathrm{C}$ NMR

$(151 \mathrm{MHz})$ in $\mathrm{CDCl}_{3}$

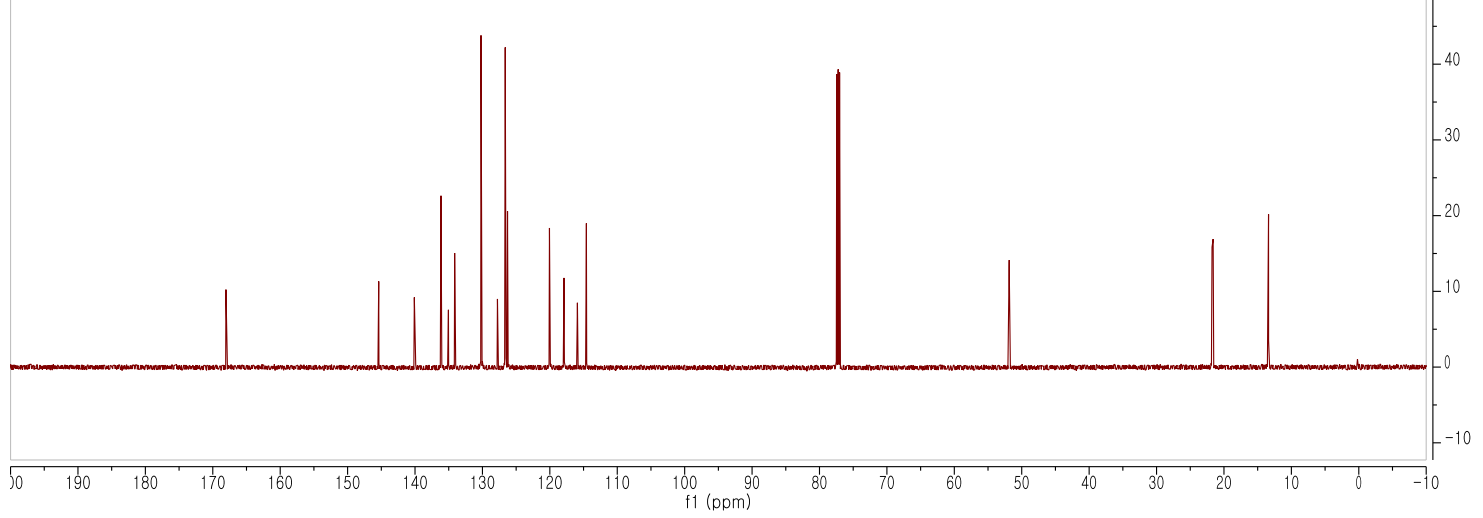



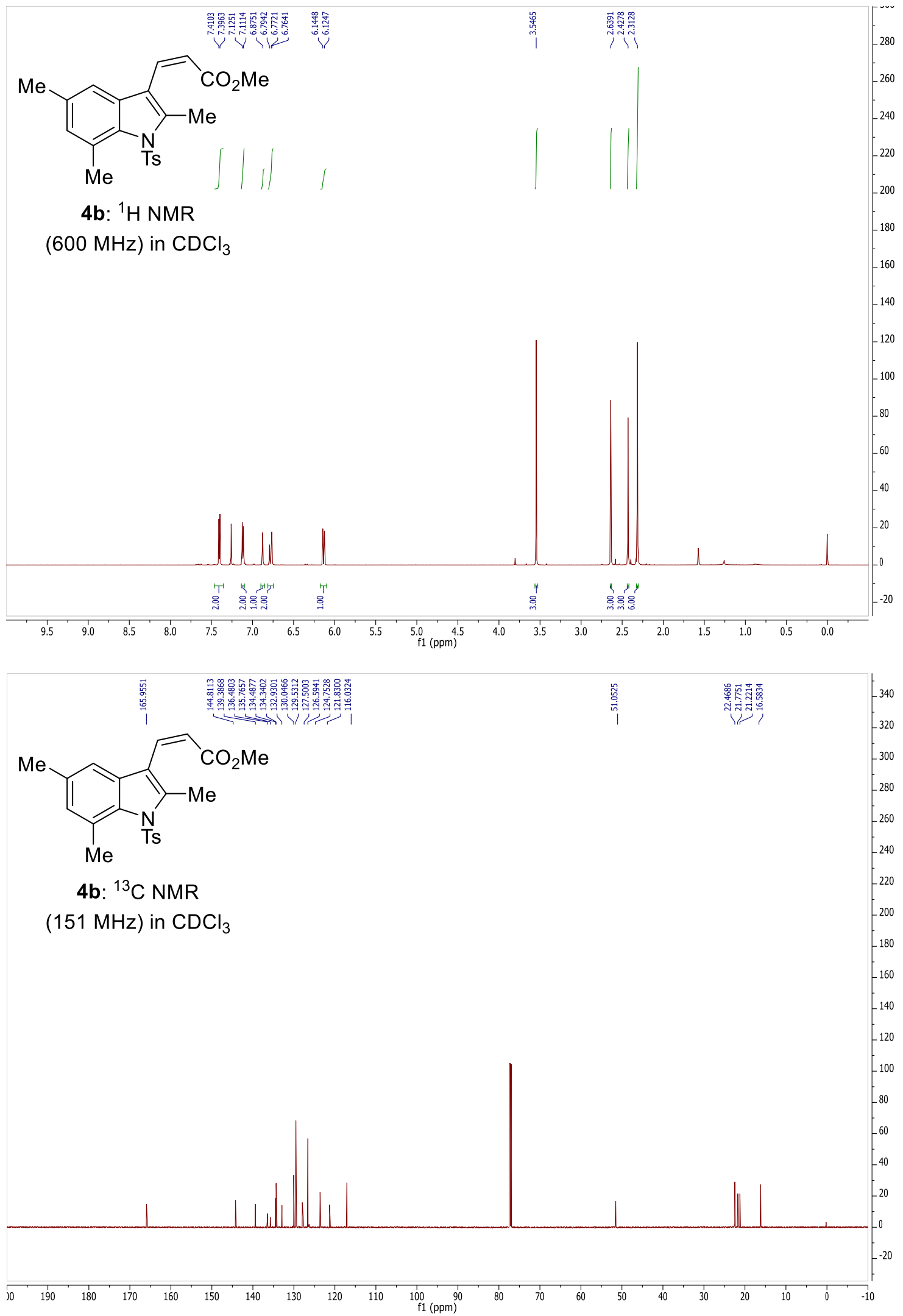

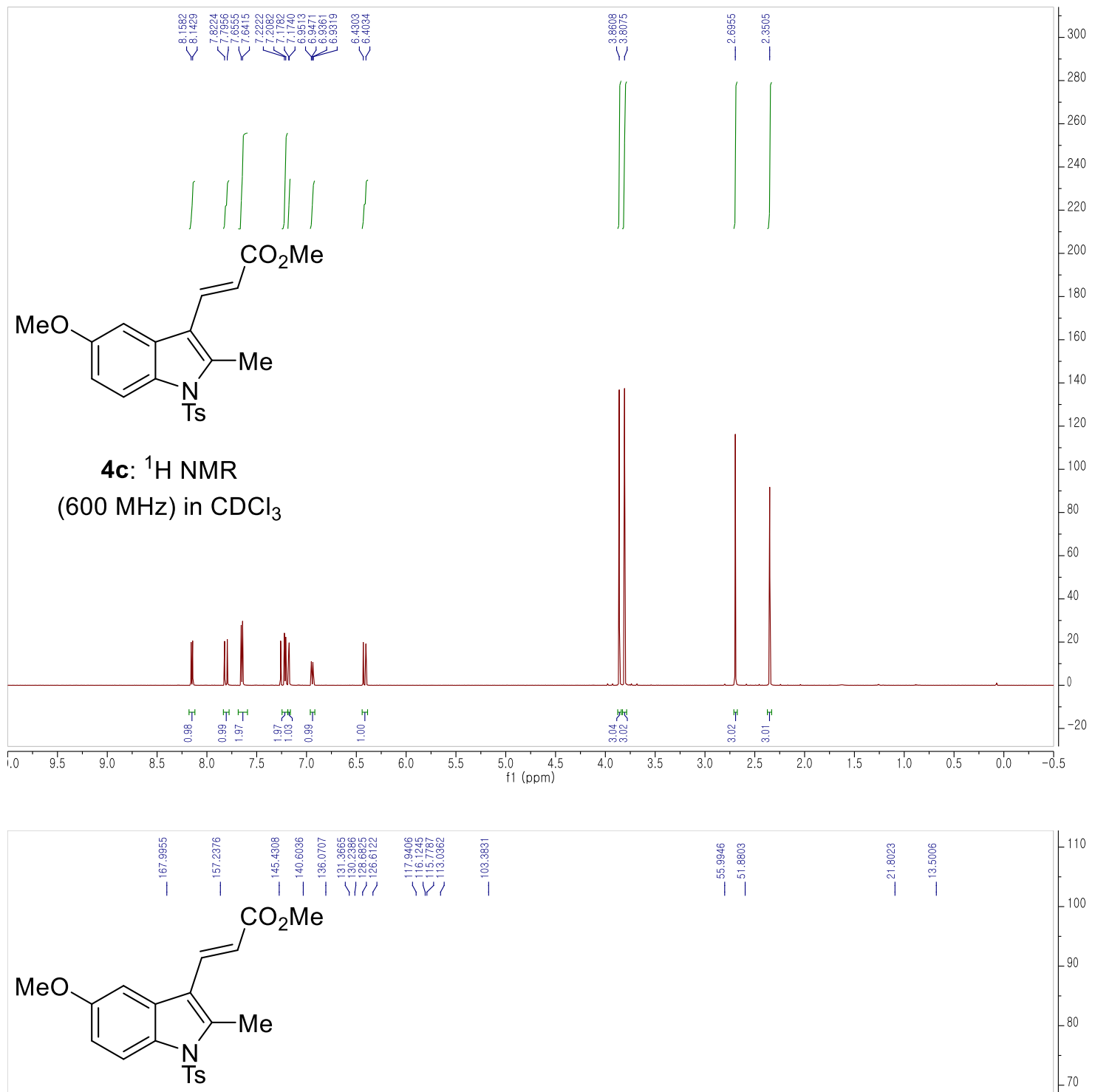

4c: ${ }^{13} \mathrm{C}$ NMR

$(151 \mathrm{MHz})$ in $\mathrm{CDCl}_{3}$

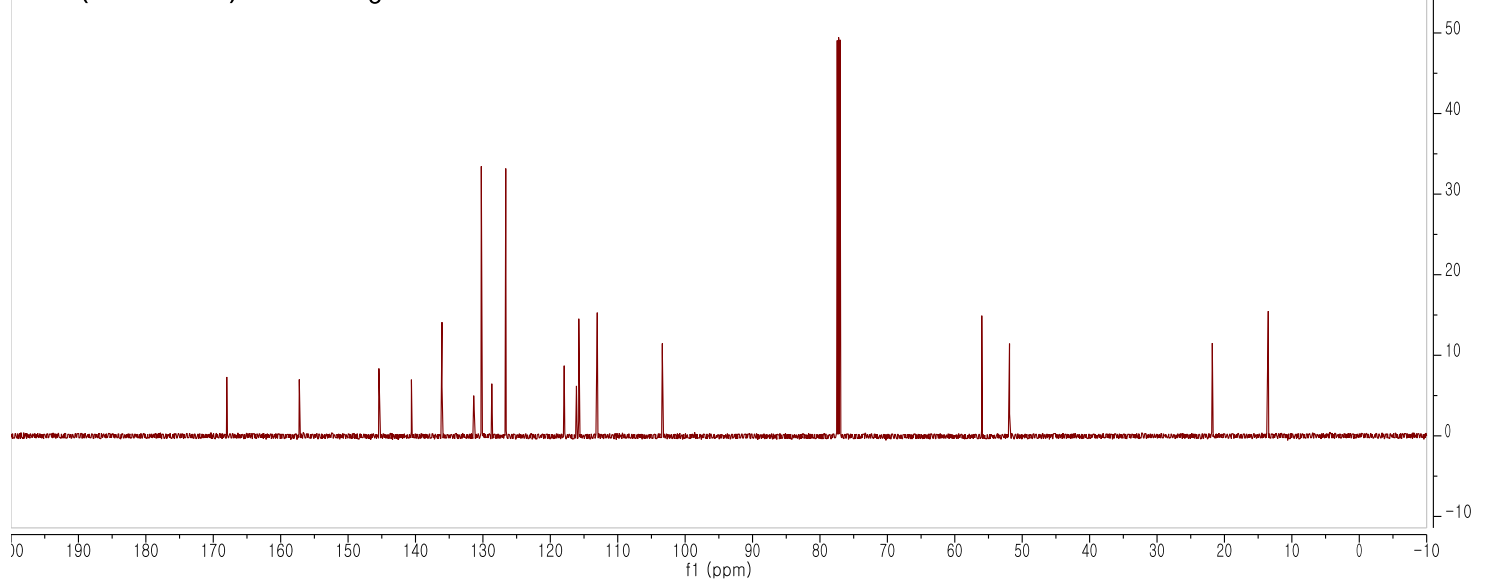




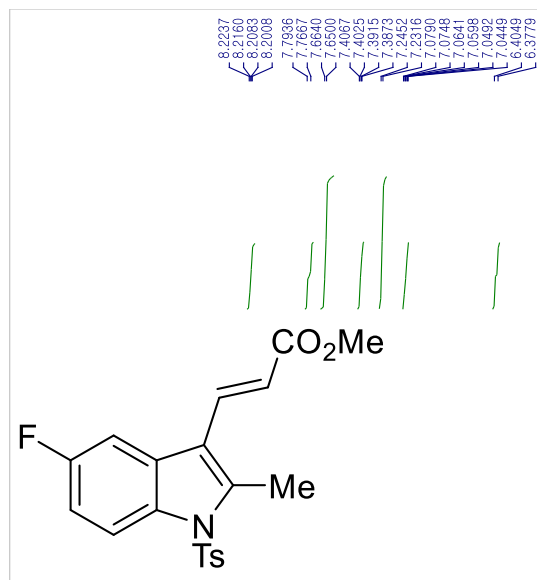

4d: ${ }^{1} \mathrm{H}$ NMR $(600 \mathrm{MHz})$ in $\mathrm{CDCl}_{3}$
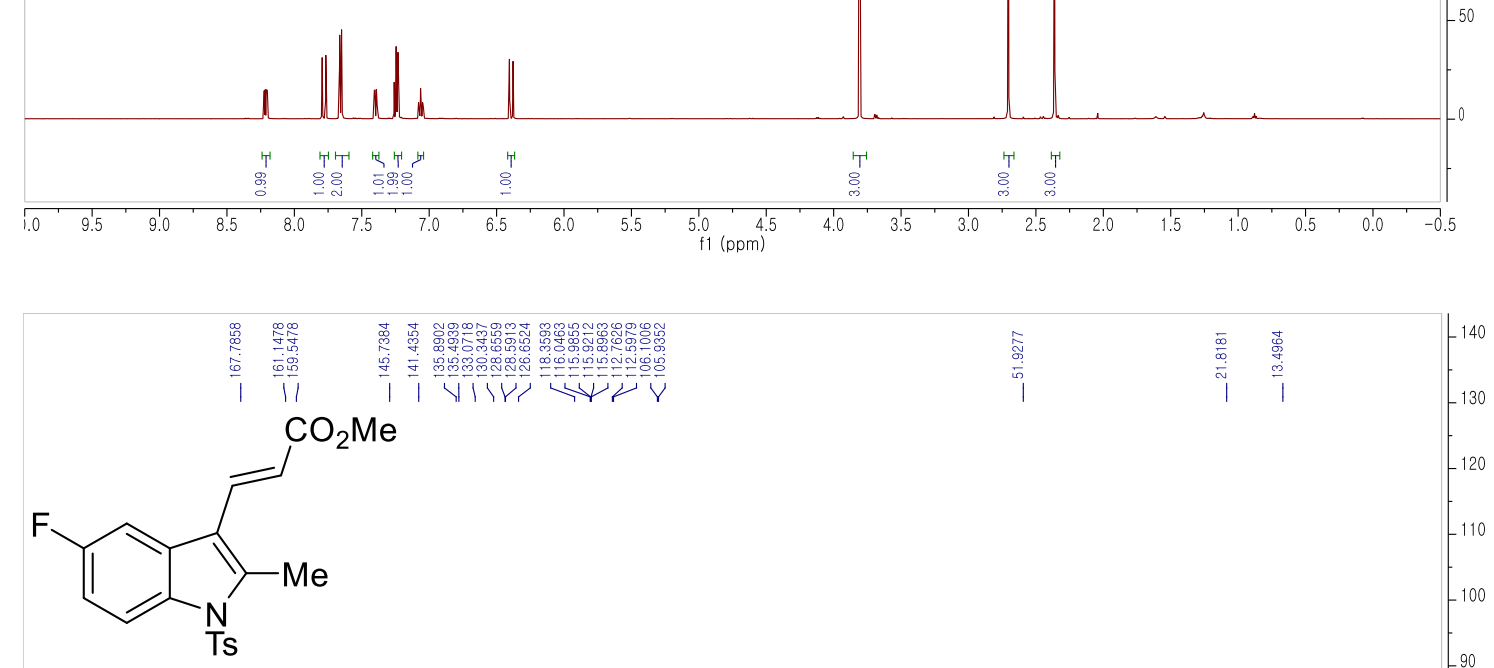

4d: ${ }^{13} \mathrm{C}$ NMR (151 MHz) in $\mathrm{CDCl}_{3}$

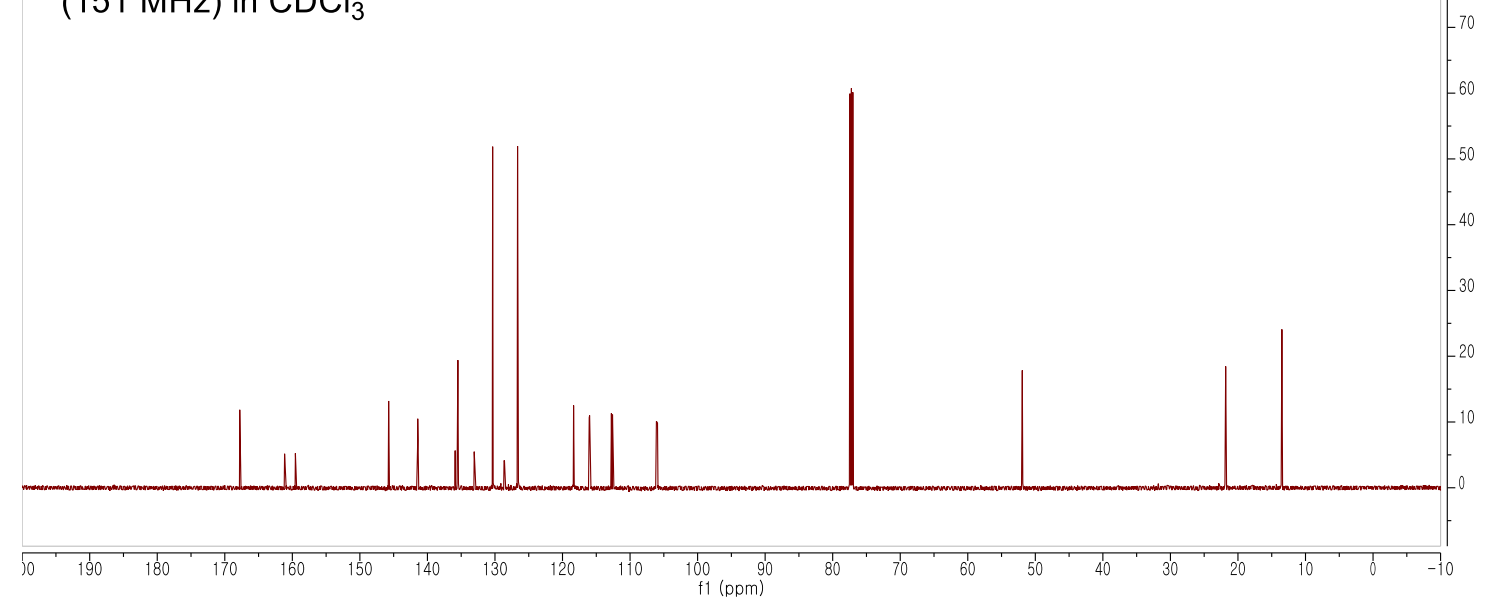



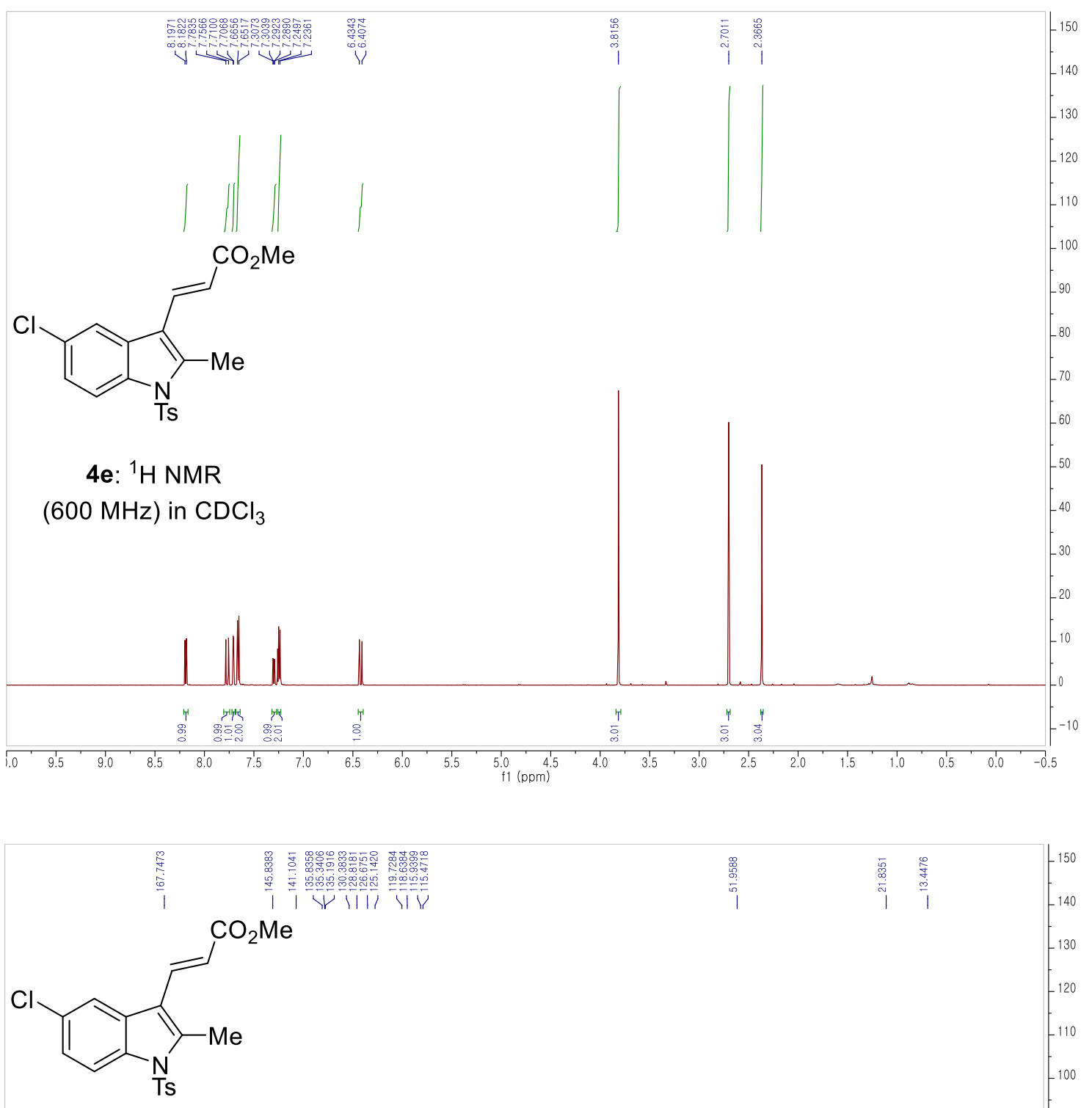

4e: ${ }^{13} \mathrm{C}$ NMR

$(151 \mathrm{MHz})$ in $\mathrm{CDCl}_{3}$

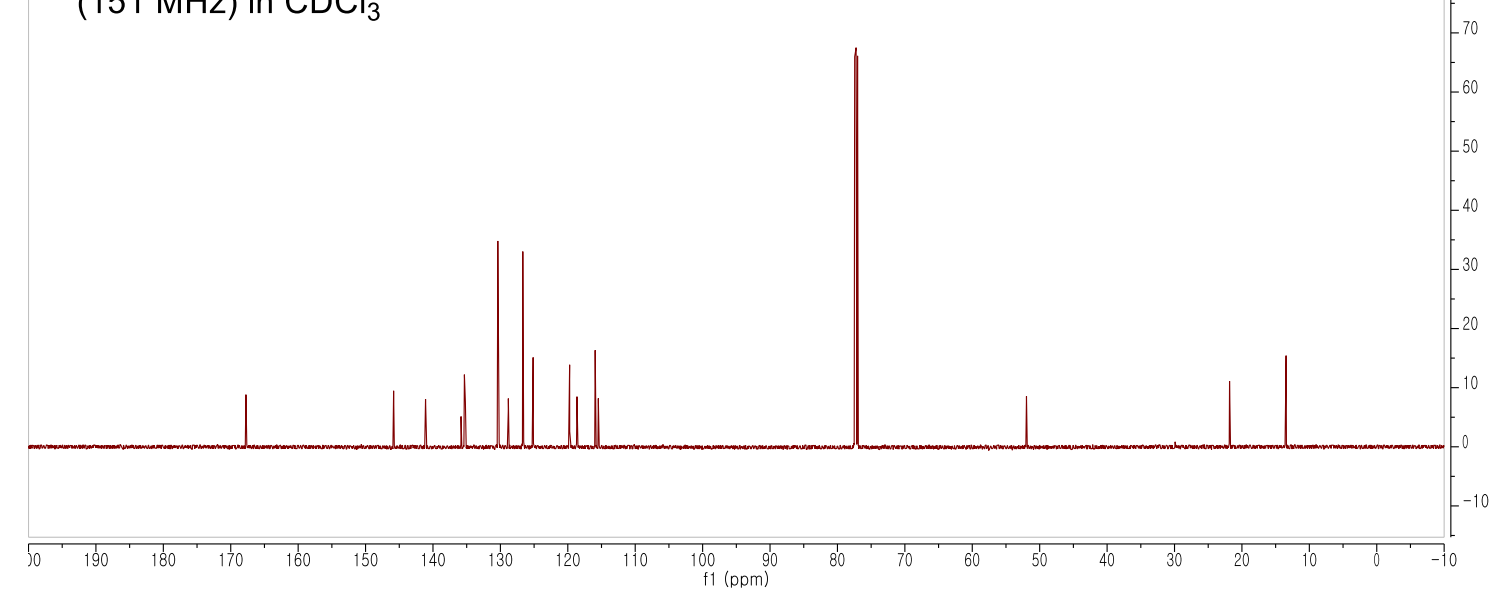




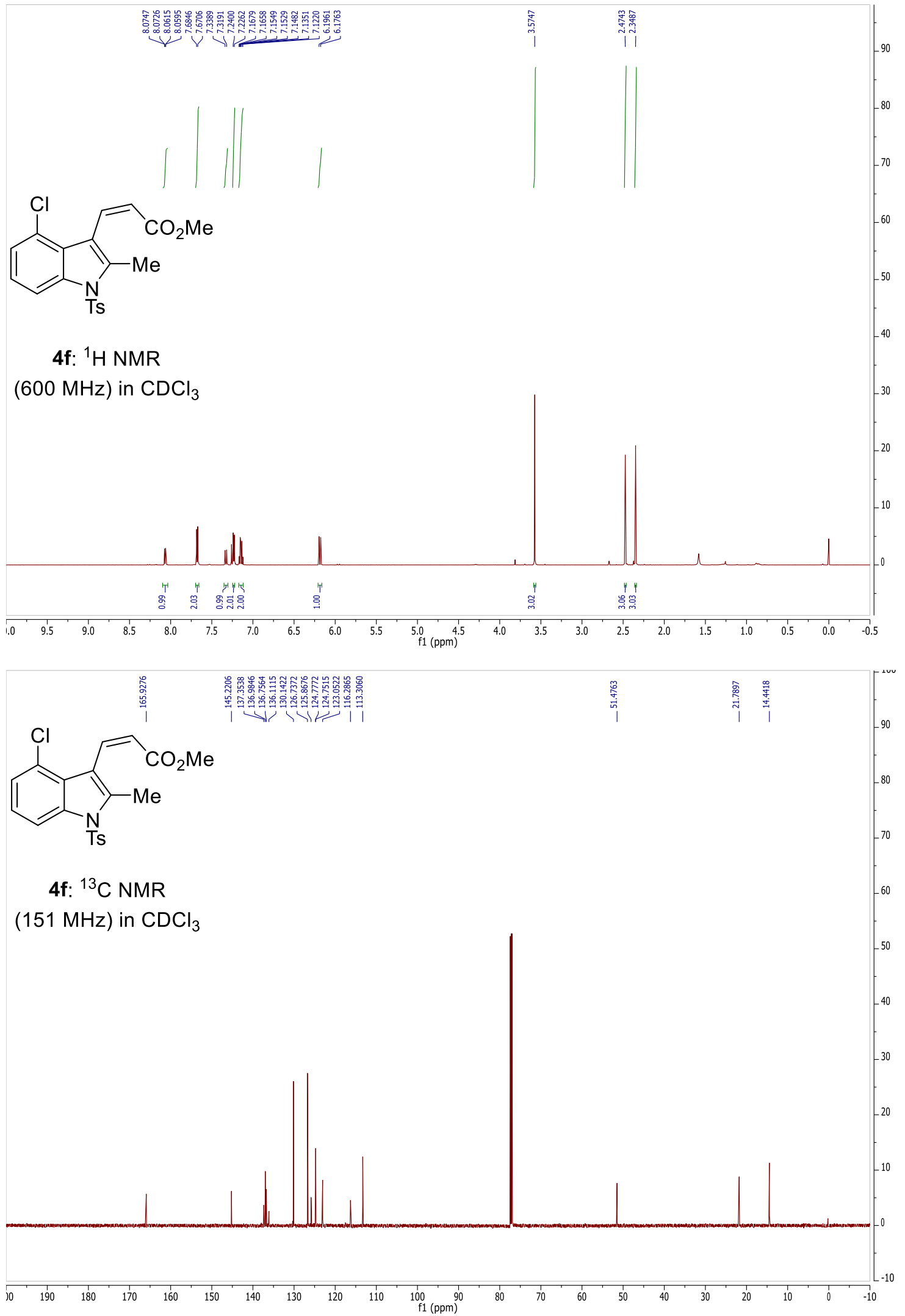



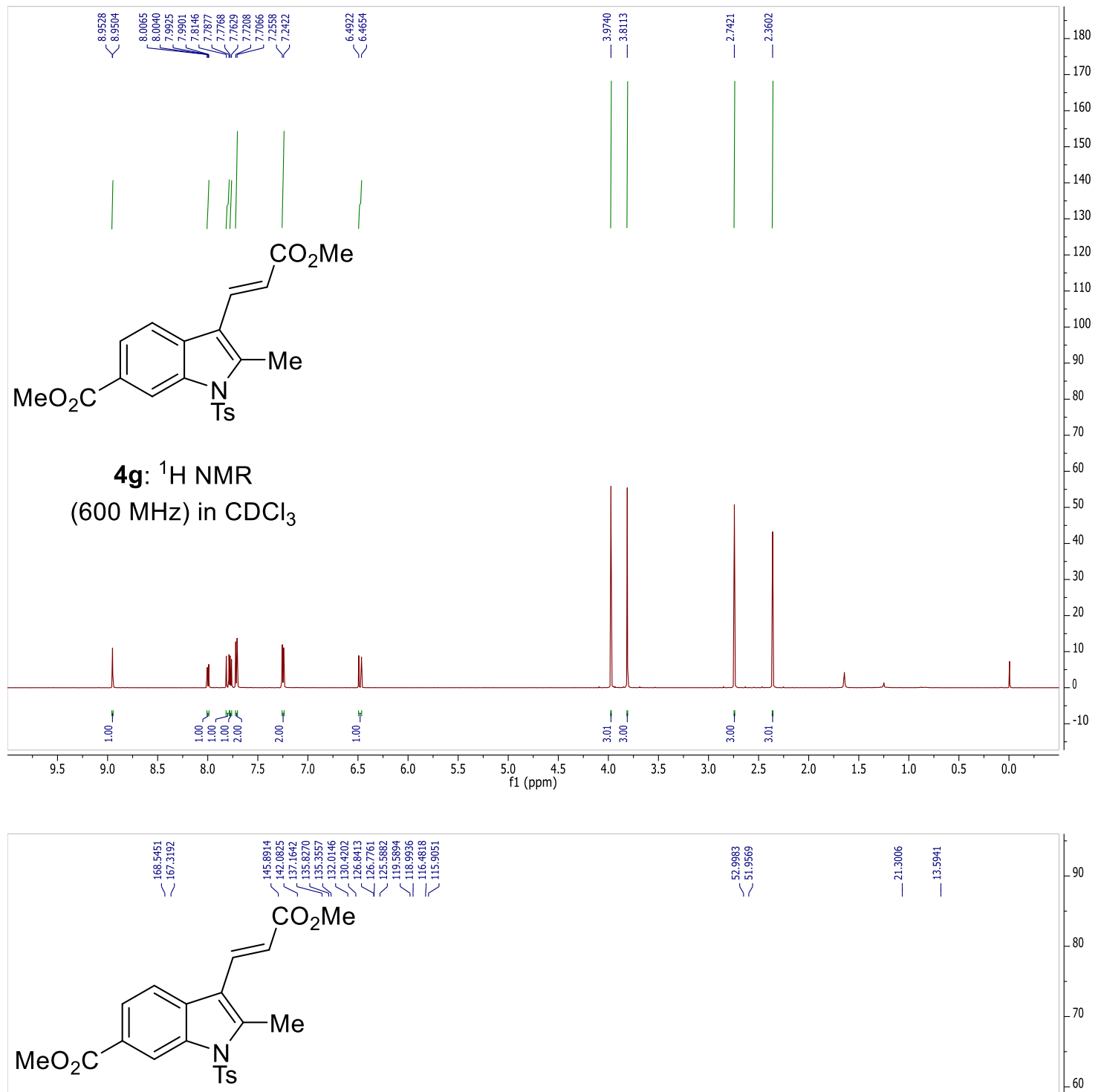

4g: ${ }^{13} \mathrm{C}$ NMR

$(151 \mathrm{MHz})$ in $\mathrm{CDCl}_{3}$

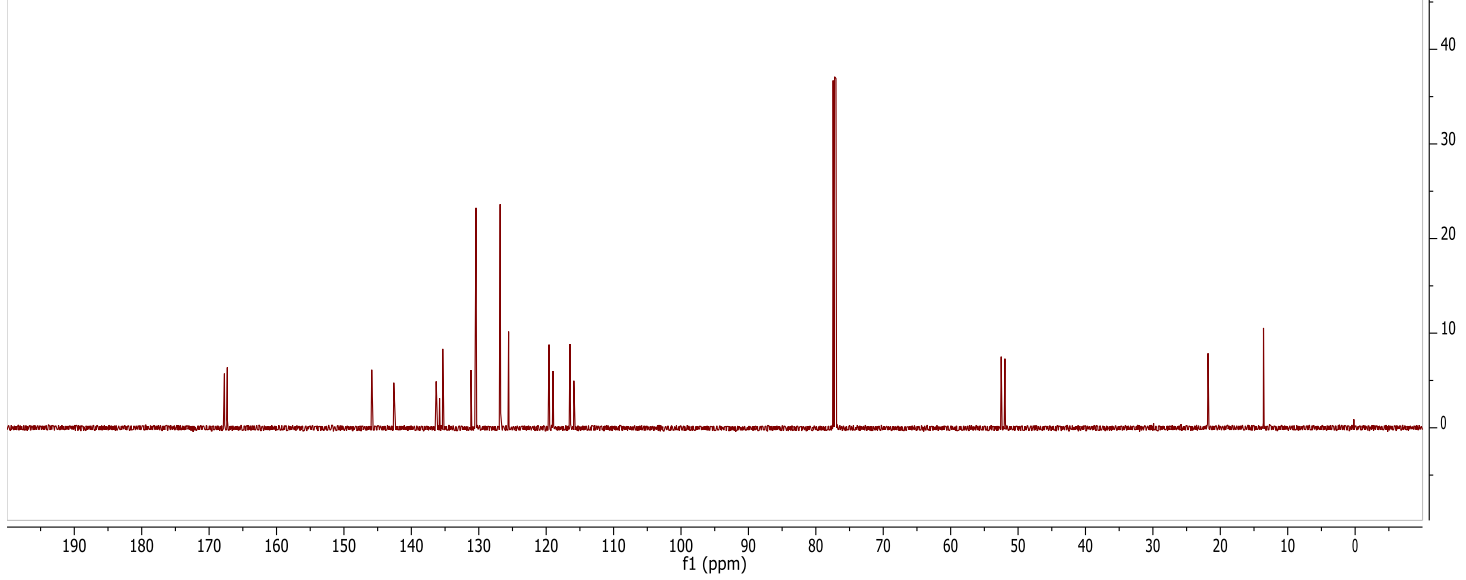



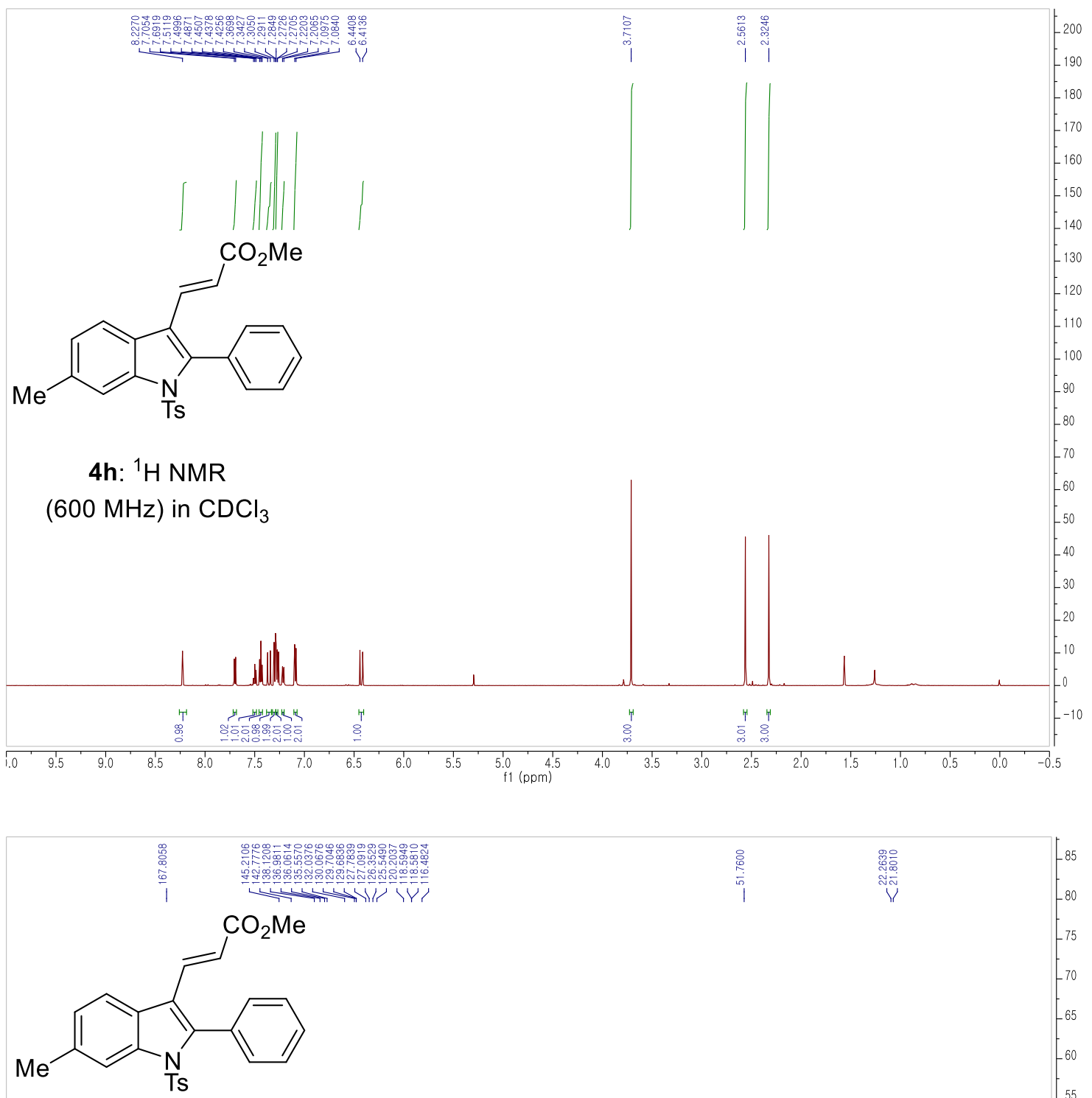

4h: ${ }^{13} \mathrm{C}$ NMR

$(151 \mathrm{MHz})$ in $\mathrm{CDCl}_{3}$

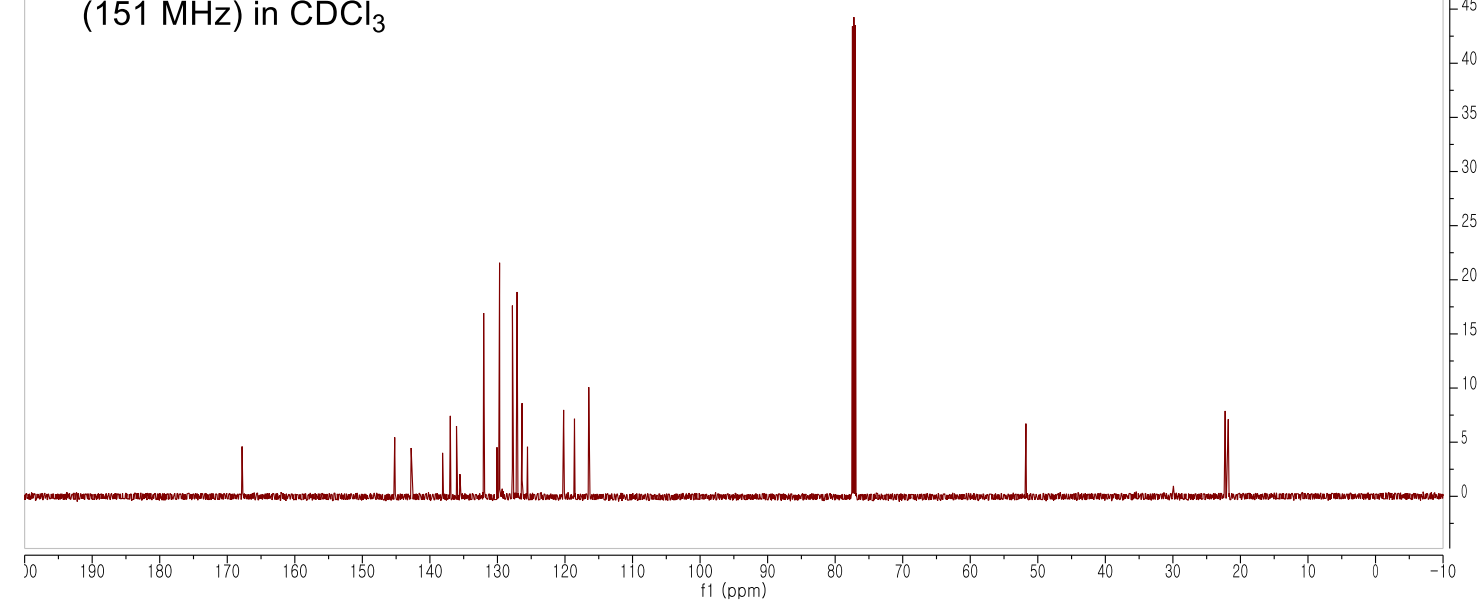



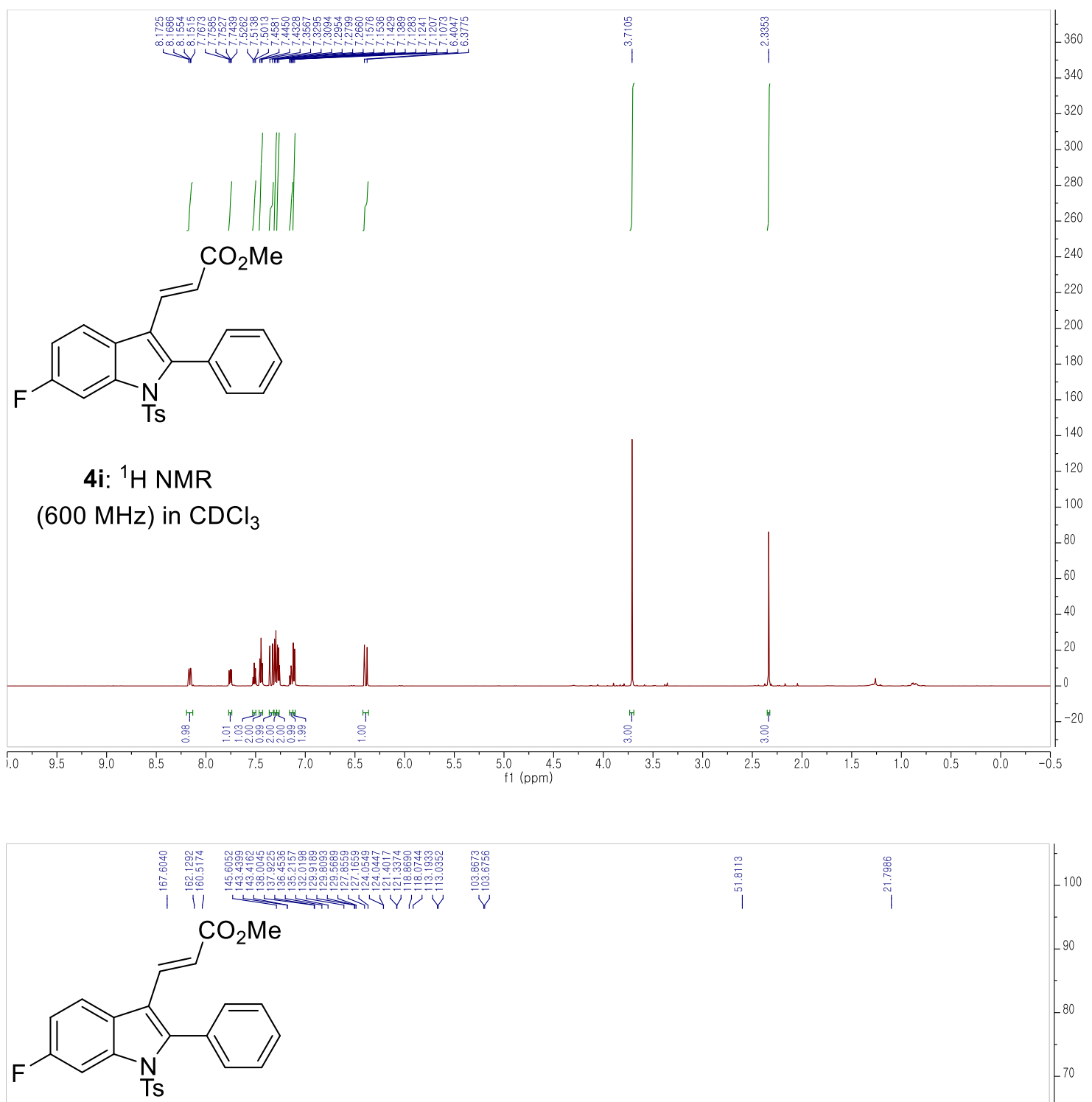

4i: ${ }^{13} \mathrm{C}$ NMR

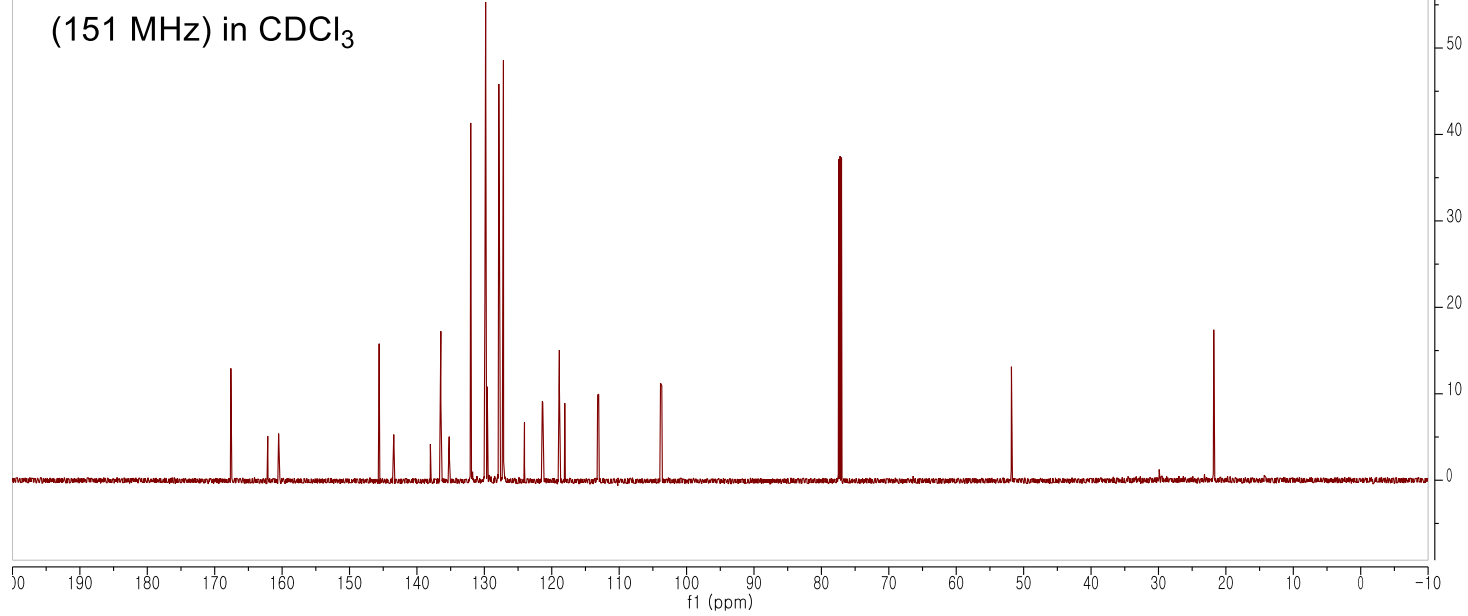



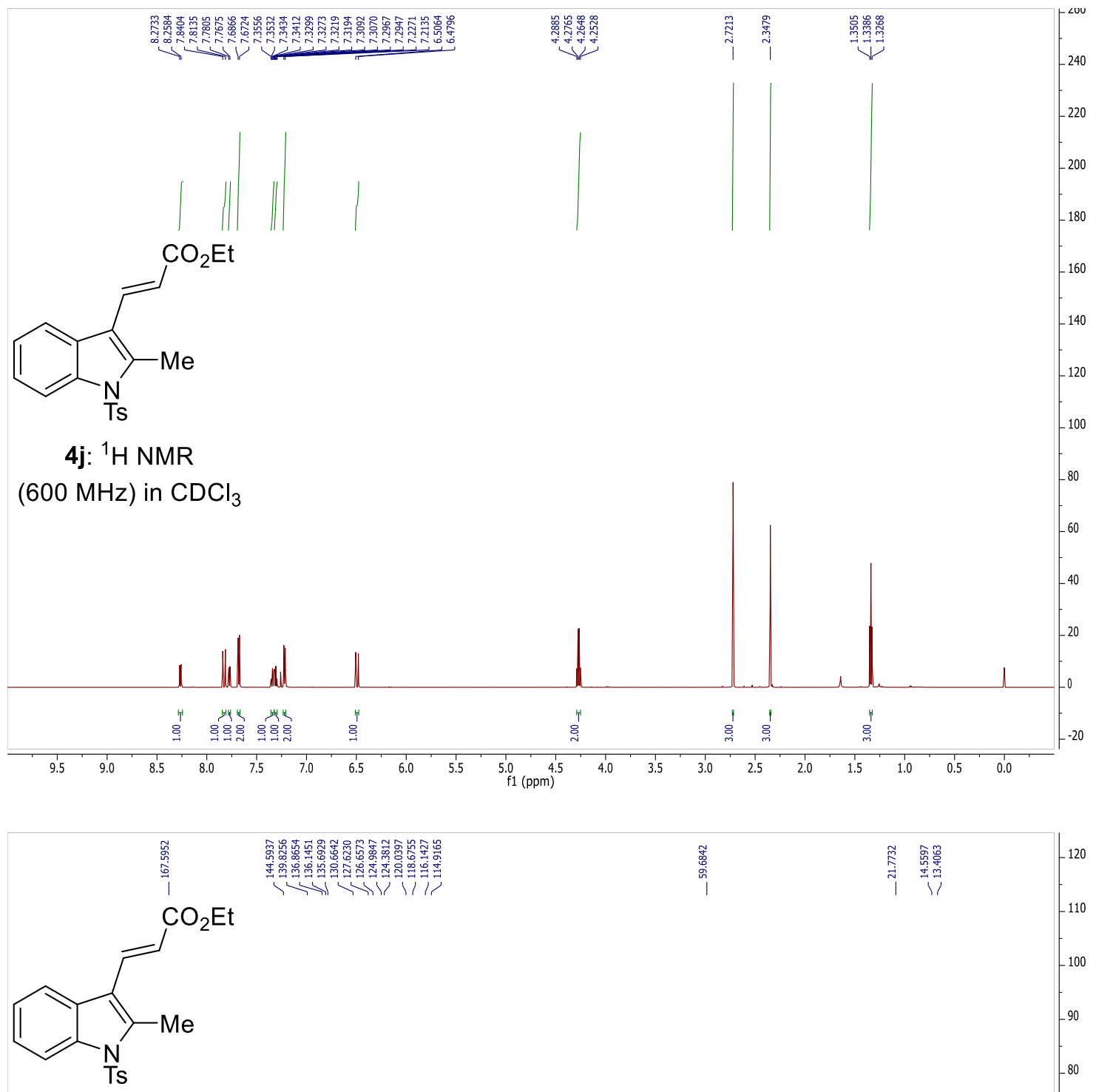

4j: ${ }^{13} \mathrm{C}$ NMR

$(151 \mathrm{MHz})$ in $\mathrm{CDCl}_{3}$

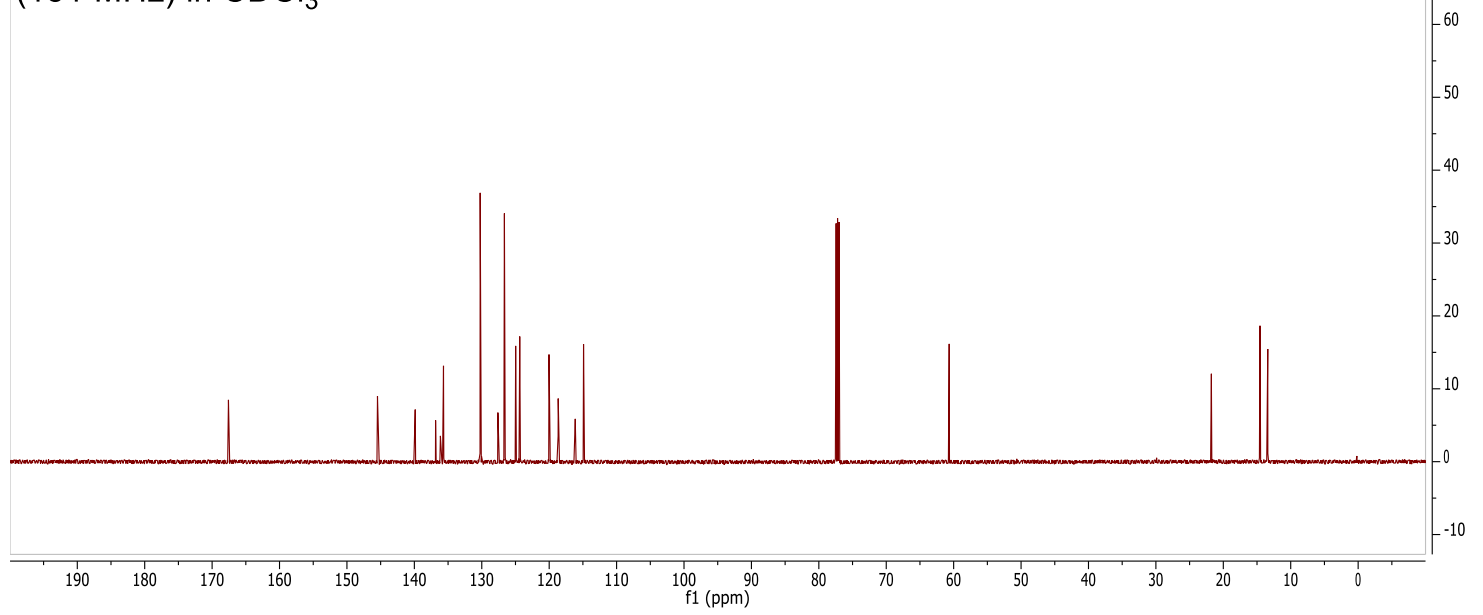



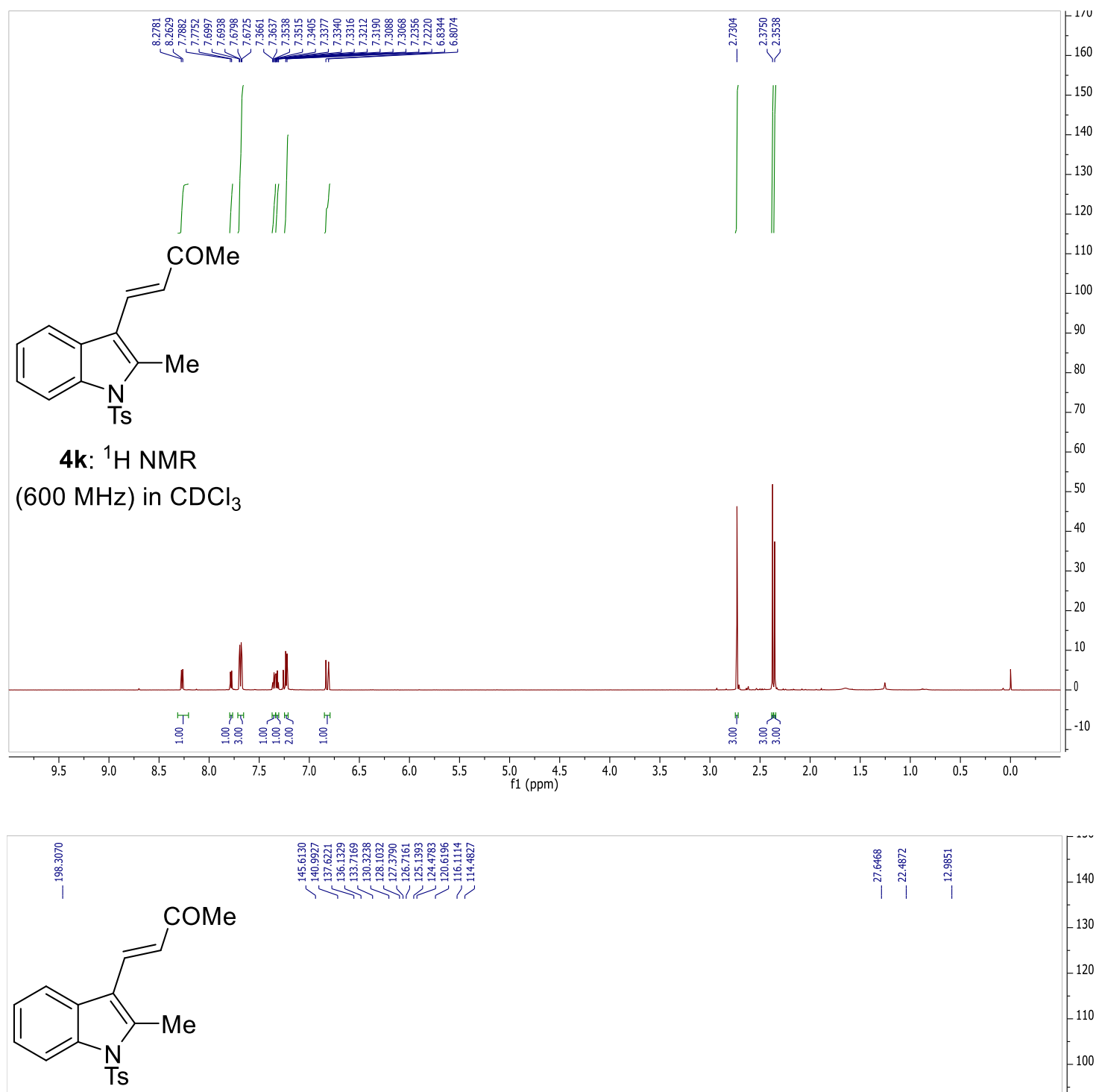

4k: ${ }^{13} \mathrm{C}$ NMR $(151 \mathrm{MHz})$ in $\mathrm{CDCl}_{3}$

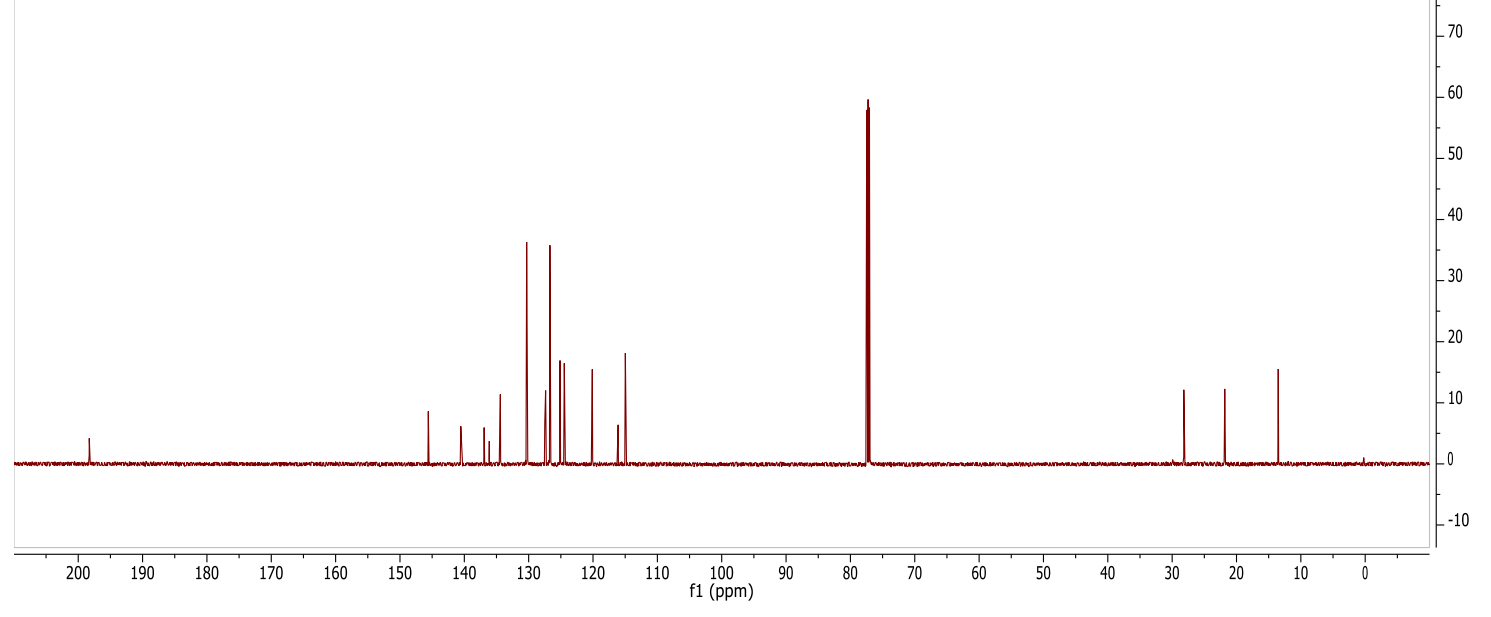




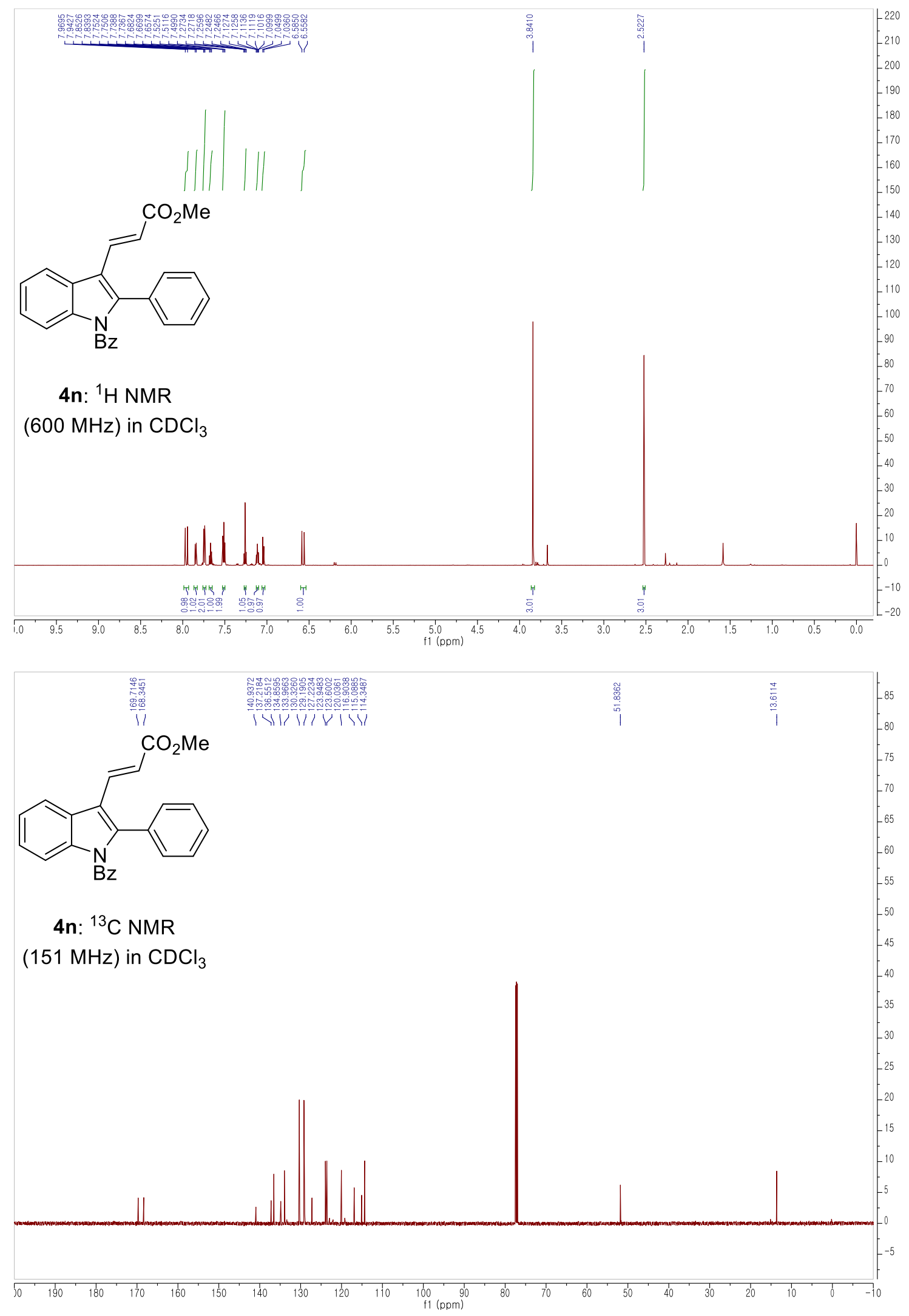



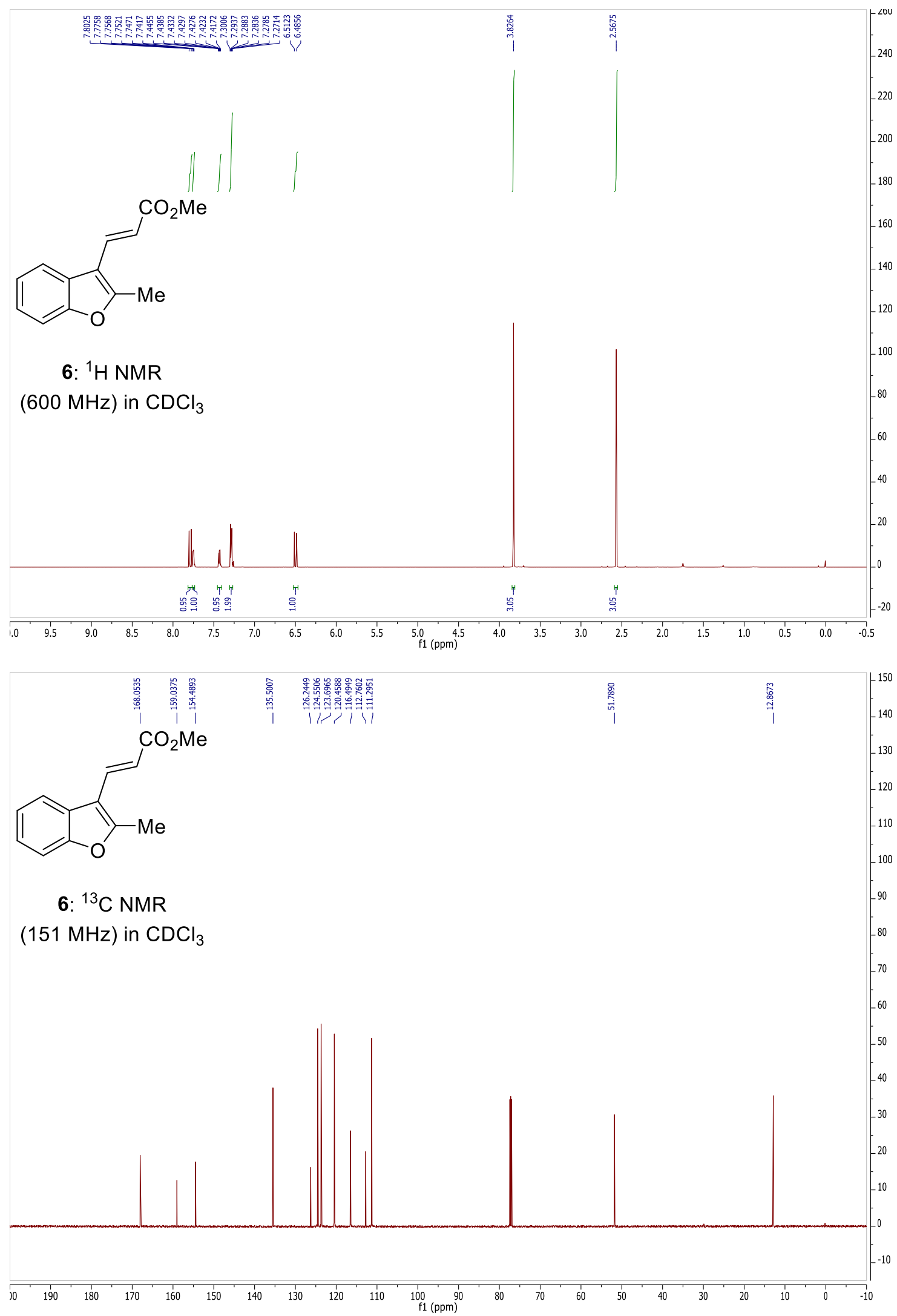

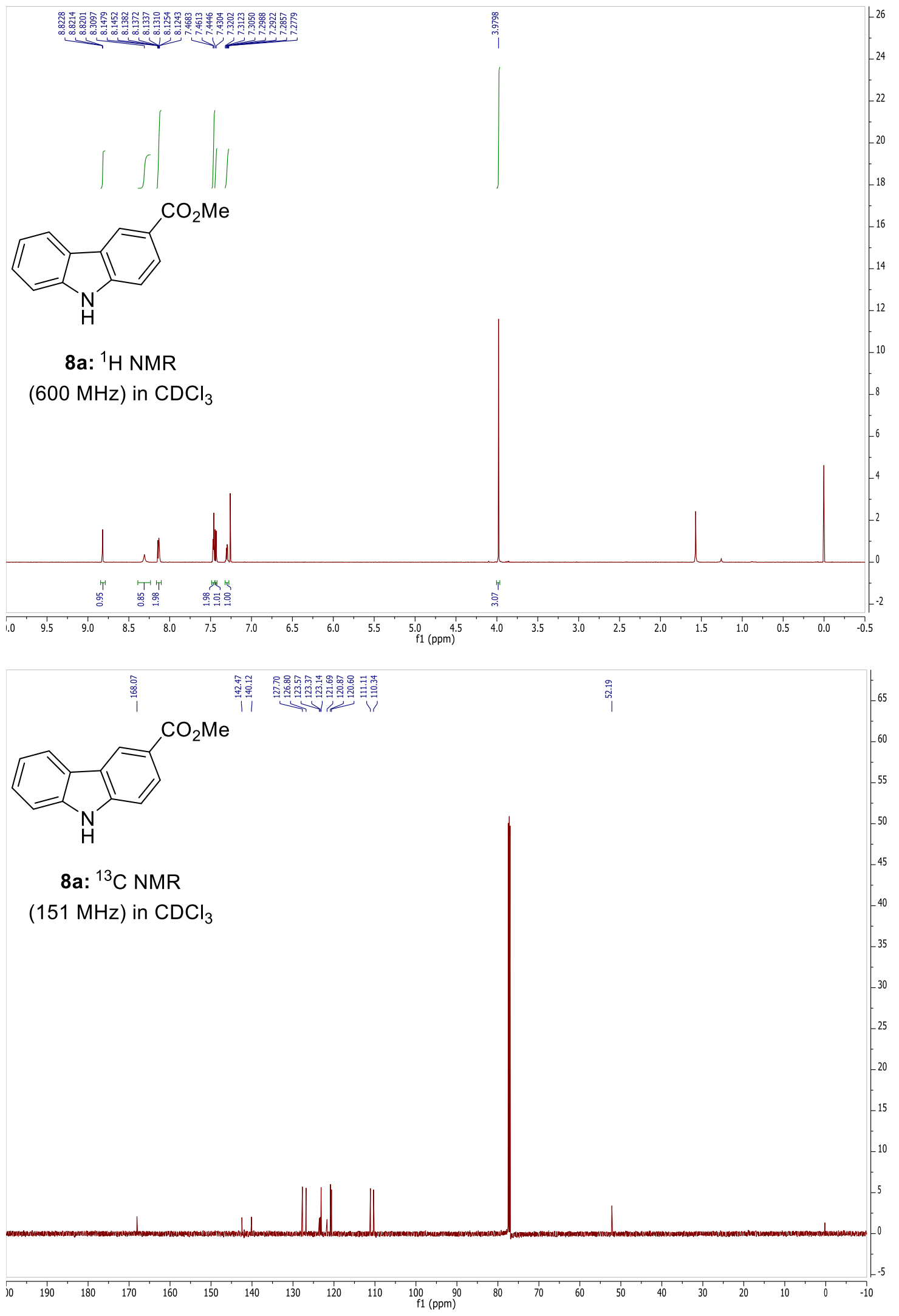

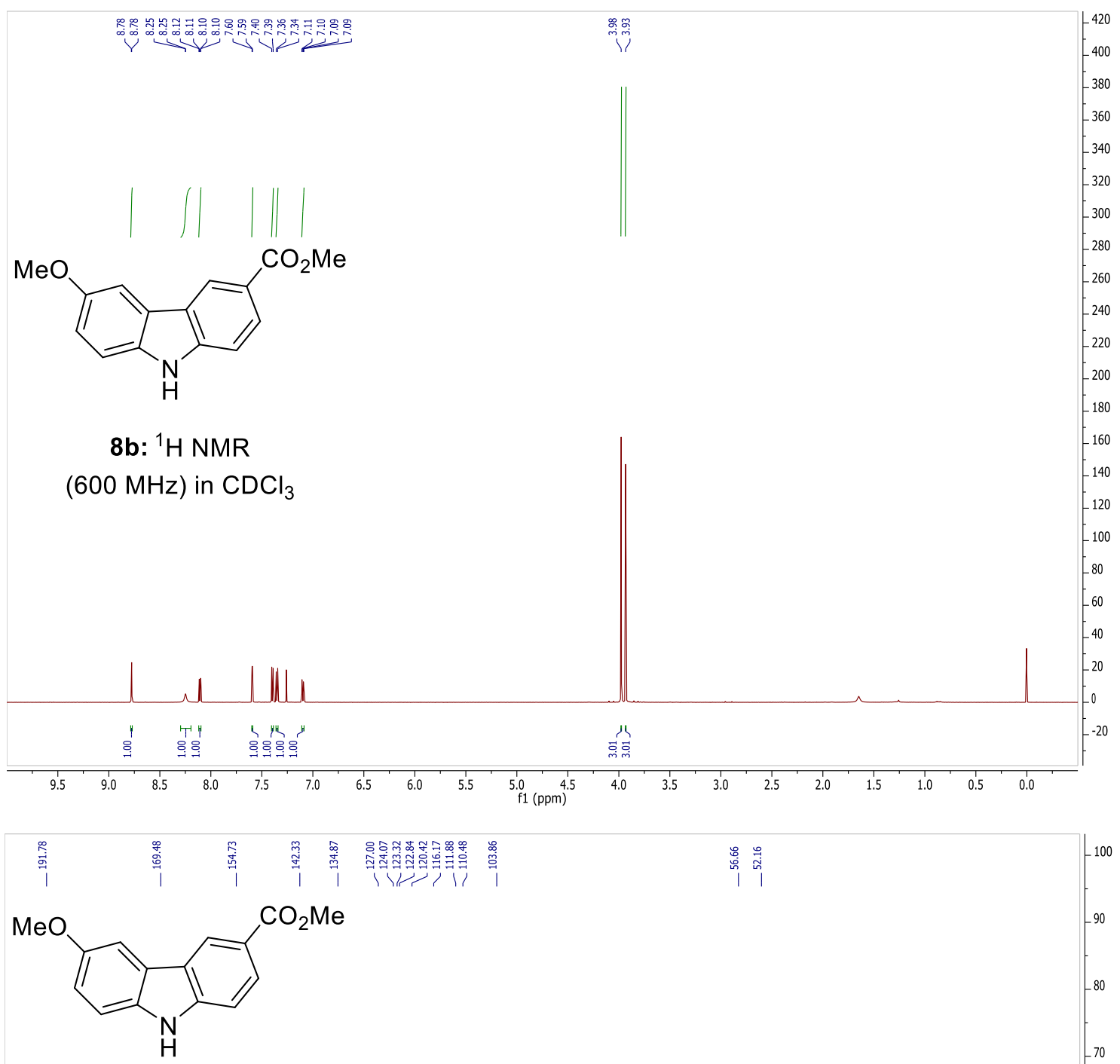

8b: ${ }^{13} \mathrm{C}$ NMR $(151 \mathrm{MHz})$ in $\mathrm{CDCl}_{3}$

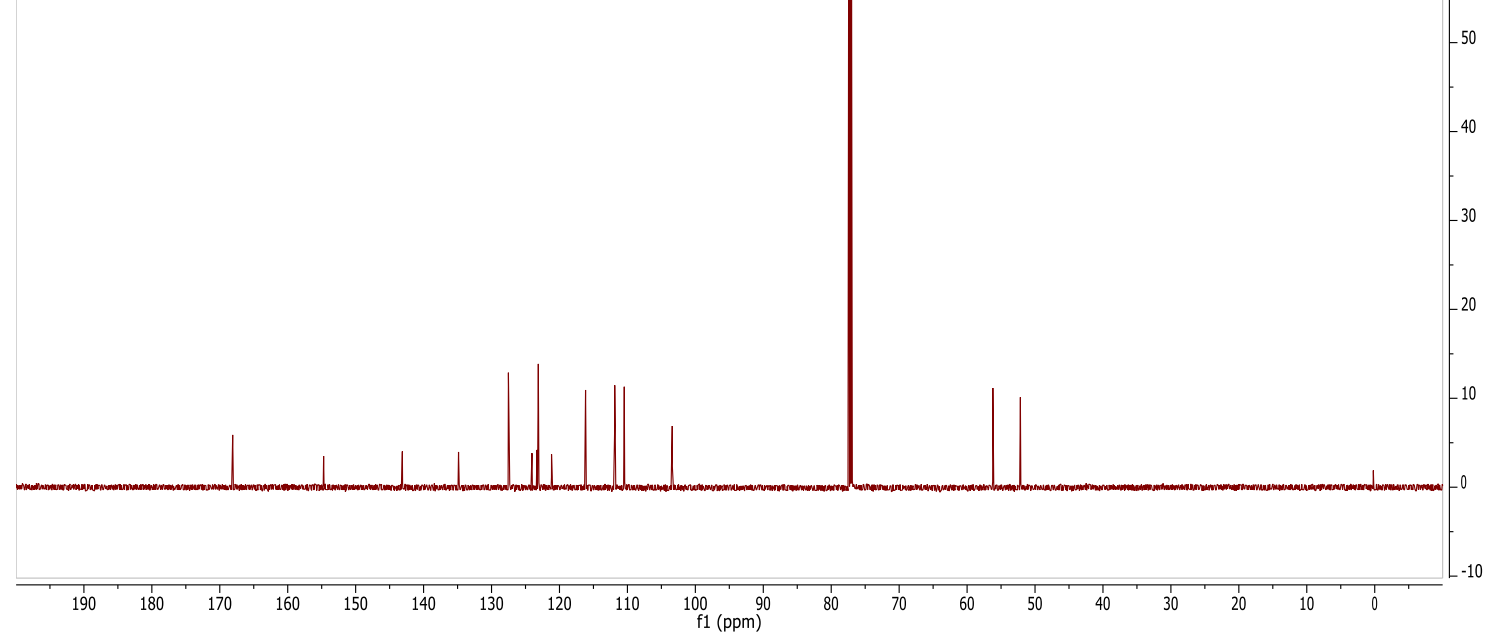



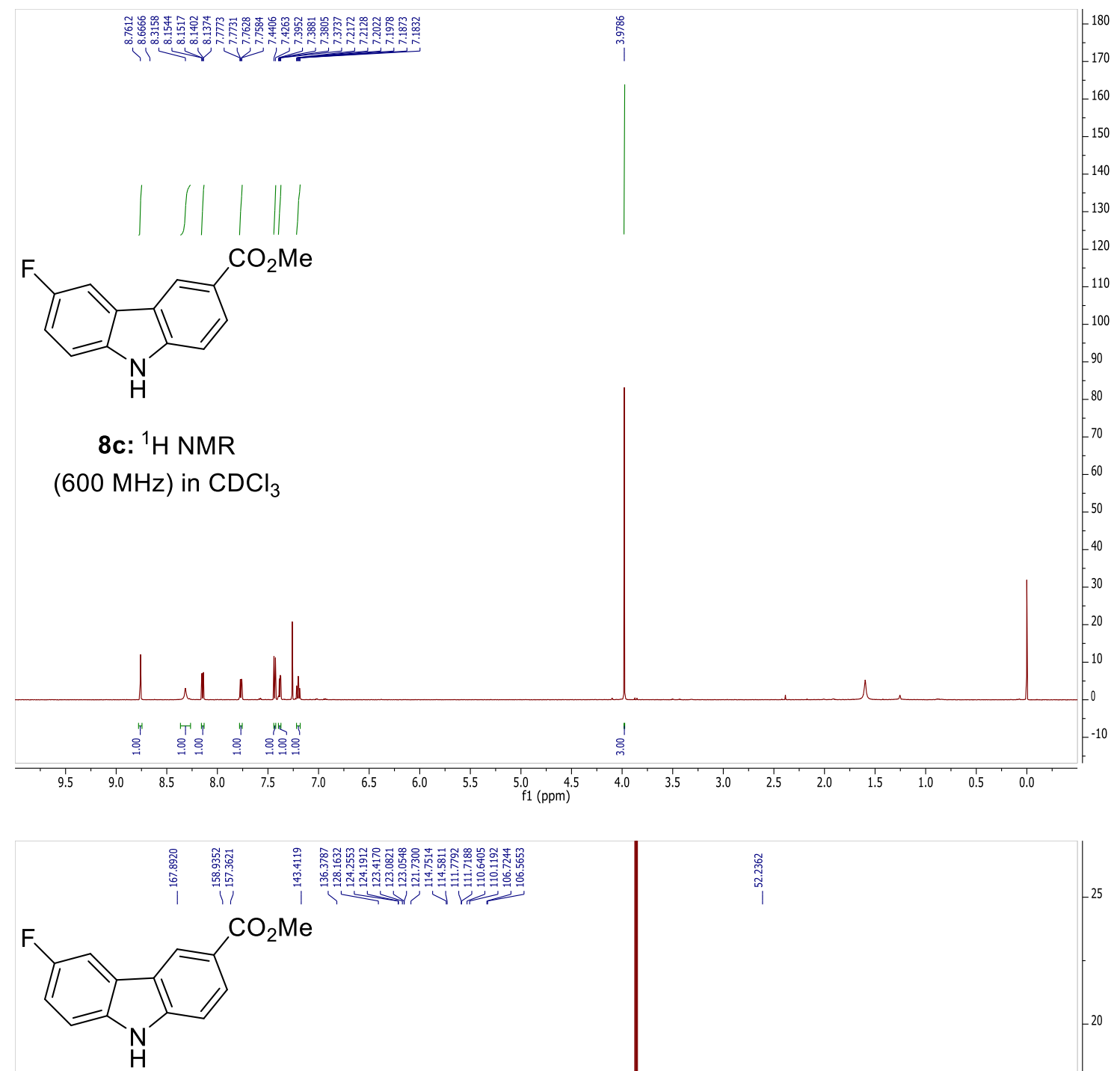

8c: ${ }^{13} \mathrm{C}$ NMR

(151 MHz) in $\mathrm{CDCl}_{3}$

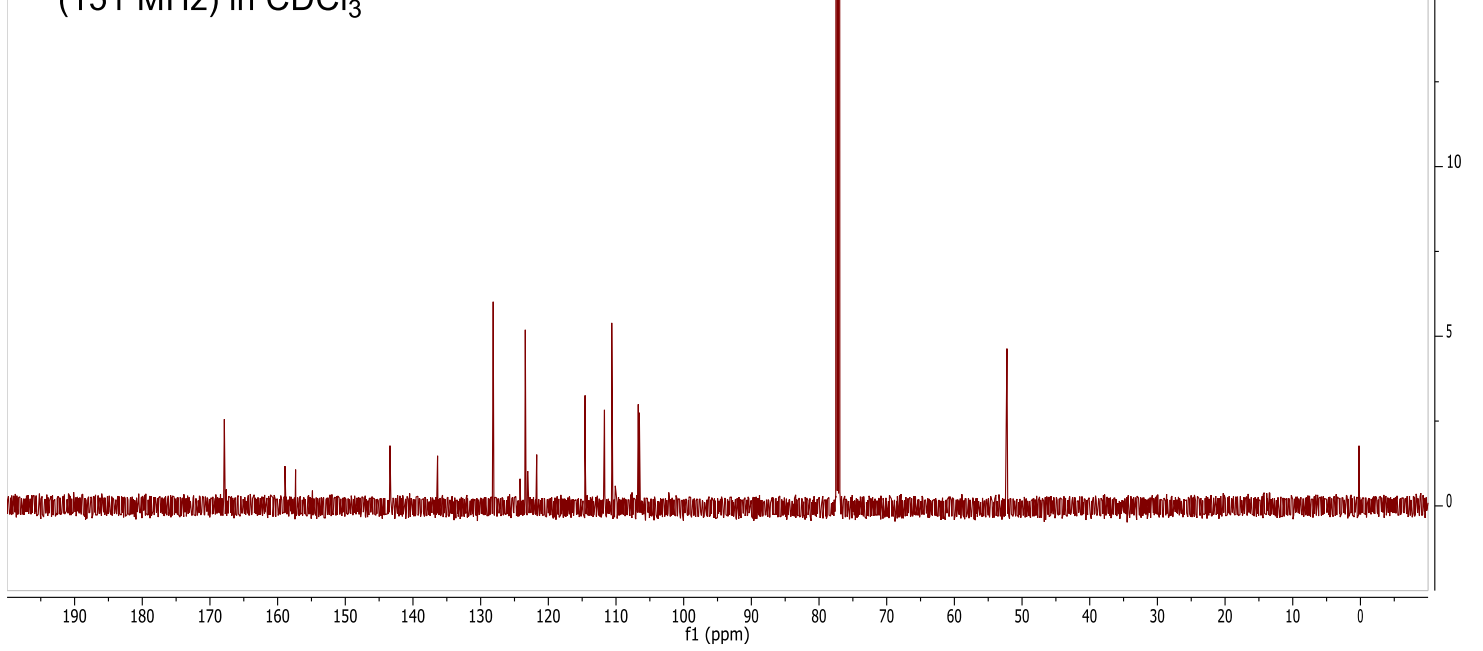

